\title{
DARK MATTER AND COLLIDER PHENOMENOLOGY OF LARGE ELECTROWEAK SCALAR MULTIPLETS
}

\author{
by \\ Terence Pilkington \\ B.Sc., M.Sc.

\begin{abstract}
A thesis submitted to the
Faculty of Graduate and Postdoctoral Affairs

in partial fulfillment of the

requirements for the degree of

Doctor of Philosophy

in Physics.
\end{abstract}
Department of Physics
Carleton University

(C) August 2016

Terence Pilkington

OtTAWA

ONTARIO 



\begin{abstract}
The Universe must contain some form of dark matter (DM), based on many astrophysical and cosmological observations. The Standard Model of particle physics (SM) does not contain a DM candidate. We may extend the SM by the addition of a single large electroweak complex scalar multiplet. These multiplets may have at most 8 members. Based on the isospin and hypercharge assignments, and the form of the scalar potential, the models we examine in this thesis have either 6 or 8 members. One of the newly-introduced particles is a DM candidate. We use the following theoretical considerations and experimental results to constrain the parameter space of these models: perturbative unitarity of scattering; stability of the scalar potential; direct searches for new physics at the LHC; electroweak observables $(S T U)$; and decays of the Higgs boson. We then compute the relic abundance in the models to determine the viability of our DM candidate. Finally, we examine the prospects of discovery at direct detection experiments. We find that these models may form part of the total DM content in the mass range $80-1000 \mathrm{GeV}$, and will begin to be probed in the direct detection experiments currently under construction. For the mass range $\gtrsim 5 \mathrm{TeV}$, we must also consider the effects of co-annihilation and Sommerfeld enhancement. In this range, our DM candidate may be all of the DM, but will require a subsequent generation of direct detection experiments to probe its parameter space.

Keywords: DARK MATter; Beyond the Standard Model; LARGe EleCTROWEAK SCALAR MULTIPLET;
\end{abstract}




\section{Statement of originality}

Chapter 2 contains work originally published in Ref. [1]. In this chapter, the author was responsible for verifying the calculation of the multiplet's maximum hypercharge and size performed by Katy Hartling and Heather Logan.

Chapter 3 contains work originally published in Ref. [2] and Ref. [3]. In this chapter, the calculation of the mass mixing matrix was originally performed by Kevin Earl and verified by the author. The calculation of the Feynman rules was independently perform by the author and Katy Hartling. The calculation of the maximum mass splitting and the re-parameterization was performed by the author (the form of the re-parameterization was suggested by Travis Martin and Heather Logan). The code to calculate the branching ratios and plot the results was written by the author.

Chapter 4 contains work originally published in Ref. [2]. The determination of the constraint on the bare mass term was performed by Heather Logan. The method to avoid alternate minima was suggested by Heather Logan, and the calculation performed by the author. The perturbative unitarity constraints on $\lambda_{2,3,4}$ was performed by Katy Hartling. The renormalization group equations were calculated by the author.

Chapter 5 contains work originally published in Ref. [2] and Ref. [3. The code used to generate the collider events was written by the author. The choice of scan points was done by the author and Travis Martin. The analysis of collider events for 
the direct search constraints was performed by Travis Martin. The calculation of the $S T U$ parameters and $h \rightarrow \gamma \gamma / Z \gamma$ was performed by Katy Hartling.

Chapter 6 contains work originally published in Ref. [2] and Ref. [4]. The calculation of the relic abundance and direct detection cross sections in the weak-scale DM mass region was performed by the author. The code used to generate points in the allowed regions was written by the author. In the multi-TeV DM mass region, the determination of the co-annihilation channels, their cross sections, and relic density was performed by the author. The calculation of the Sommerfeld enhancement was performed by the author. The calculation of the direct detection cross section was performed by the author.

Appendix E contains work originally published in Ref. [5]. The calculation of the relic abundance and direct detection cross sections was independently performed by the author and Heather Logan. The calculation of the $S T U$ constraints was performed by Katy Hartling. The calculation of the Higgs decay constraint was performed by Heather Logan and verified by the author. 


\section{Acknowledgements}

There are many people I need to thank for their support and contributions to this thesis. First and foremost, my advisor Heather Logan, whose patient explanations and confidence in my abilities allowed me to pursue this dark matter phenomenology research. I must single out Katy Hartling, who was more than a colleague and collaborator, but also a close friend. The Herzbergers trivia team consisting (at one time or another) of Matt, Katie, Katy, Kyle, Carolyn, Jesse, Dan, François, Rouz, and Dal. The friends I made at TASI 2014 (Alex, Anders, Anthony, Gabriel, J.J., Josh, Nicolas, Ray, and Sean) will continue to be a connection to other specializations within physics. Those I met at conferences and workshops provided feedback and suggestions on my work. My family and friends provided vast amounts of emotional support throughout my studies.

I dedicate this thesis to my grandparents Harold and Frances Tarris, who instilled in me a desire to figure out how things work and to do that precisely. 


\section{Contents}

Abstract

Statement of originality

$\begin{array}{lll}\text { Acknowledgements } & \text { iv }\end{array}$

List of figures viii

List of tables xii

List of acronyms, symbols, and constants

Chapter 1. Introduction 1

1.1. The Standard Model of particle physics 2

1.2. Dark matter 8

1.3. Experimental pathways to the observation of dark matter 14

1.4. Large electroweak scalar multiplets 17

\begin{tabular}{lll} 
Chapter 2. Large electroweak scalar multiplets & 20 \\
\hline
\end{tabular}

2.1. Groups and the Standard Model 21

2.2. The electroweak sector: Isospin and hypercharge 28

2.3. Extending the electroweak sector 30

2.4. Large electroweak scalar multiplets 32

2.5. Constraints on multiplet size and hypercharge 33

2.6. Chapter summary 43 
Chapter 3. $Z_{2}$-symmetric models 45

3.1. Model description 46

3.2. Re-parameterization of the models 52

3.3. Branching ratios and preferred decay modes 57

3.4. Chapter summary 64

Chapter 4. Theoretical constraints on $Z_{2}$-symmetric models 66

4.1. Symmetry of the potential 67

4.2. Stability of the potential and bounded from below conditions 69

4.3. Unitarity constraints 77

4.4. Renormalization group equations 81

4.5. Chapter summary 95

Chapter 5. Collider constraints on $Z_{2}$-symmetric models 96

5.1. Particle colliders and event simulation 97

5.2. Event simulation 101

5.3. Direct search constraints from LHC data 103

5.3.1. Results for the $n=6$ model 111

5.3.2. Results for the $n=8$ model 113

5.3.3. Summary of direct search constraints 118

5.4. Effects of new physics in loops affecting SM processes 119

5.4.1. STU 121

5.4.2. $h \rightarrow \gamma \gamma$

5.4.3. $h \rightarrow Z \gamma$

5.5. Chapter summary 135

Chapter 6. Dark matter constraints on $Z_{2}$-symmetric models 136 
6.1. Thermal history of the Universe 137

6.2. Freeze-out 141

6.3. Relic density of weak-scale large multiplet DM 146

6.4. Relic density of multi-TeV large multiplet DM 152

6.4.1. Co-annihilation 153

6.4.2. Sommerfeld enhancement 158

6.4.3. Combining co-annihilation with Sommerfeld enhancement 168

6.5. Dark matter direct detection 174

6.5.1. Direct detection of weak-scale large multiplet DM

6.5.2. Direct detection of multi-TeV large multiplet DM 183

6.6. Chapter summary 186

Chapter 7. Summary 187

Appendix A. Feynman rules of the large electroweak scalar multiplet models 190

\begin{tabular}{lll}
\hline Appendix B. & Isospin combinations for quartic terms $[\Sigma \Sigma]_{T}$ & 200
\end{tabular}

\begin{tabular}{|lll} 
Appendix C. & Larger-dimensional representations of $S U(2)$ & 208
\end{tabular}

Appendix D. One-loop RGEs in the large electroweak scalar multiplet models 213

Appendix E. $U(1)$-symmetric models 218

\begin{tabular}{ll}
\hline References & 228 \\
\hline
\end{tabular} 


\section{List of figures}

$1.1 \quad$ Particles that make up the Standard Model.

$1.2 \quad$ SM predictions compared with experimental measurements.

$1.3 \quad$ Sketch of a galactic rotation curve. 9

1.4 Gravitational lensing of a distant object by an intervening mass.

1.5 Matter content of the Universe.

1.6 Possible DM-SM interactions.

1.7 Comic illustrating why calling our models "large electroweak scalar multiplets" provides a complete description of the model in its name. 18

$2.1 \quad$ Rotations and reflections of an equilateral triangle. 21

$2.2 \quad$ Argand diagram showing the unitarity circle. 34

$2.3 \quad$ Feynman diagrams contributing to the annihilation of two multiplet members into two gauge bosons. 35

$2.4 \quad$ Kinematics for the process $\Sigma \Sigma^{*} \rightarrow V^{\mu} V^{\nu}$.

$3.1 \quad$ Mass vs. $\lambda_{3,4}$ in the large multiplet models.

$3.2 \quad$ Mass vs. charge plots for $m_{\zeta^{0, r}}=180 \mathrm{GeV}$ in both $n=6,8$. 53

$3.3 \quad$ Plots showing the translation between $\lambda_{3,4}$ and $R_{1,2}$ for $n=6$. 56

$3.4 \quad$ Plots showing the translation between $\lambda_{3,4}$ and $R_{1,2}$ for $n=8$. 57

3.5 Dominant decay modes of $\zeta^{0, i}$ in the $n=6$ model. 58 
$3.6 \quad$ Dominant decay modes of $H_{2}^{+}$in the $n=6$ model. 59

$3.7 \quad$ Dominant decay modes of $H_{2}^{++}$in the $n=6$ model. 59

3.8 Dominant decay modes of $\zeta^{0, i}$ in the $n=8$ model. 60

3.9 Dominant decay modes of $H_{2}^{+}$in the $n=8$ model. 60

3.10 Dominant decay modes of $H_{2}^{++}$in the $n=8$ model. 61

3.11 Dominant decay modes of $H_{2}^{+3}$ in the $n=8$ model. 61

3.12 Detailed plots of mass splittings, coupling strengths, and branching ratios for decays of $\zeta^{0, i}$ in $n=8$. 63

3.13 Detailed plots of mass splittings, coupling strengths, and branching ratios for decays of $H_{2}^{++}$in $n=6$. 64

$4.1 \quad$ Plot of a simple quartic scalar potential. $\quad 69$

$4.2 \quad$ Plot of a scalar potential, $V(\Phi, \Sigma)$. 73

$4.3 \quad$ Feynman diagram for the electron propagator correction. 81

4.4 Plot of the renormalization group equation running for the SM gauge couplings. 83

$4.5 \quad$ Feynman diagrams showing the contributions from scalar loops. 87

$4.6 \quad$ Plot showing the Landau pole location for the scalar quartic couplings in $Z_{2}$-symmetric models. 92

$4.7 \quad$ Normalized difference between Landau pole and initial conditions as a function of $\left|\lambda_{4}\right|$. 93

$5.1 \quad$ Simplified view of particle detectors used in collider experiments. 98

$5.2 \quad$ Possible visible missing transverse momentum interactions. 103 
5.3 Sample Feynman diagram for a collider process in the large multiplet models.

5.5 Exclusion regions in the $n=6$ model from all examined LHC8 analyses. 116 5.6 Exclusion regions in the $n=8$ model from all examined LHC8 analyses. 117 5.7 Comparison between QCD loop-order calculations of gluon fusion Higgs production.

5.8 Feynman diagram of the scattering of light fermions showing the one-loop fermion correction to the gauge boson propagator.

5.9 Feynman diagrams showing the one-loop contributions of the large multiplets to the gauge boson propagators.

5.10 Allowed regions in the $\lambda_{3} \lambda_{4}$-plane with $S T U$ bounds imposed.

5.11 Mass splitting between $\zeta^{0, r}$ and $\zeta^{0, i}$ with unitarity and $S T U$ bounds. 128

5.12 Sample Feynman diagrams showing the SM contribution to $h \rightarrow \gamma \gamma$. 129

5.13 Sample Feynman diagrams showing the large multiplet contribution to

$h \rightarrow \gamma \gamma$.

129

5.14 Allowed $\lambda_{2}$ values as a function of $m_{\zeta^{0, r}}$ with $S T U$ and $h \rightarrow \gamma \gamma$ constraints imposed.

132

5.15 Feynman diagrams showing the large multiplet contribution to $h \rightarrow Z \gamma$. 133

6.1 The CMB temperature power spectrum as a function of multipole moment.141

$6.2 \quad$ Freeze-out of a generic WIMP as a function of $m / T$.

6.3 Feynman diagrams for the computation of the relic abundance of $\zeta^{0, r}$. 147

6.4 DM fraction for $\zeta^{0, r}$ as a function of $m_{\zeta^{0, r}}$. 
6.5 DM fraction for $\zeta^{0, r}$ as a function of $m_{\zeta^{0, r}}$ with the LHC8 constraints.

6.6 DM fraction as a function of $m_{\zeta^{0, r}}$ in the multi-TeV region.

6.7 Mass splitting between $\zeta^{0, r}$ and $\zeta^{0, i}$ as a function of $m_{\zeta^{0, r}}$ in the multi-TeV region.

6.8 Feynman diagrams used in calculating the relic abundance of $H_{1}^{+1}$.

6.9 Plots showing where $\Omega_{\zeta} / \Omega_{\mathrm{DM}}=1$ incorporating co-annihilation.

6.10 Feynman diagrams corresponding to Sommerfeld enhancement.

6.11 Sommerfeld enhancement with a Coulomb potential.

6.12 Sommerfeld enhancement with a Hulthén potential.

6.13 Thermally-averaged Sommerfeld enhancement with a Hulthén potential.

6.14 Plots showing where $\Omega_{\zeta} / \Omega_{\mathrm{DM}}=1$ incorporating Sommerfeld enhancement.

6.15 Plots showing where $\Omega_{\zeta} / \Omega_{\mathrm{DM}}=1$ comparing co-annihliation and Sommerfeld enhancement.

6.16 Plots showing where $\Omega_{\zeta} / \Omega_{\mathrm{DM}}=1$ for all effects.

6.17 Plot of DM direct detection exclusion curve as a function of mass.

6.18 Feynman diagram for the scattering $\zeta^{0, r} N \rightarrow \zeta^{0, r} N$.

6.19 Direct detection predictions for the weak-scale parameter space.

6.20 Direct detection predictions for the multi-TeV parameter space.

E.1 Mass splitting in the $U(1)$-symmetric models.

E.2 DM fraction and direct detection bound for $U(1)$-symmetric models. 225

E.3 Resonance region and Higgs decay rate as a function of $\Lambda_{0}$. 


\section{List of tables}

1.1 Relative strengths of the fundamental forces. 3

$2.1 \quad$ Multiplication table for the triangle group. 22

2.2 Quantum numbers for a single generation of SM fermions. 29

$2.3 \quad$ Group representations for the SM gauge bosons. 30

$2.4 \quad$ Maximum hypercharge, weak isospin, and size of large multiplets. 44

$3.1 \quad$ Maximum mass splitting between $\zeta^{0, r}$ and $\zeta^{0, i}$.

4.1 Mass dimensions of common fields. 67

4.2 Upper bounds on the scalar quartic couplings from perturbative unitarity. 80

5.1 Spectrum and splittings for a point in the compressed-spectrum region. 109

5.2 Best-fit values for the electroweak oblique parameters from a global fit. $\quad 123$

$5.3 \quad$ Signal strengths for $h \rightarrow \gamma \gamma / Z \gamma$ relative to the SM predictions.

6.1 Total isospin and hypercharge combinations in the the SM.

6.2 Mass ranges for multi-TeV $\zeta^{0, r}$ DM. Values given in TeV.

$6.3 \quad$ Excitation and ionization light production in DM DD experiments. 177

\begin{tabular}{lll}
6.4 & Effective Higgs-quark couplings. & 180 \\
\hline
\end{tabular}

E.1 Upper limits on scalar quartic couplings in $U(1)$-symmetric models. 219 


\section{List of acronyms, symbols, and constants}

Natural units $(\hbar=c=1)$ will be used. The particle physics sign convention (+---) will be followed for the metric. Lowercase greek indices $(\mu, \nu, \ldots)$ will run over spacetime coordinates. The Einstein summation convention where repeated indices are summed over will be employed, e.g., $a_{i} b^{i}=\sum_{i=1}^{n} a_{i} b^{i}$. Values in the tables come from the Review of Particle Physics [6], measured at $Q^{2}=m_{Z}$

\begin{tabular}{|c|c|c|c|}
\hline Acronym & Description & Acronym & Description \\
\hline $\mathrm{SM}$ & Standard Model of particle physics & EM & Electromagnetism \\
\hline EW & Electroweak & QED & Quantum Electrodynamics \\
\hline QCD & Quantum Chromodynamics & CERN & $\begin{array}{l}\text { Conseil européen pour la } \\
\text { recherche nucléaire }\end{array}$ \\
\hline LEP & Large Electron-Positron collider & LHC & Large Hadron Collider \\
\hline ATLAS & A Toroidal LHC ApparatuS & CMS & Compact Muon Solenoid \\
\hline $\mathrm{eV}$ & Electronvolt $\left(1 \mathrm{GeV}=10^{9} \mathrm{eV}\right)$ & $\mathrm{NP}$ & New physics \\
\hline $\mathrm{DM}$ & Dark matter & WIMP & $\begin{array}{l}\text { Weakly interacting massive } \\
\text { particle }\end{array}$ \\
\hline LUX & $\begin{array}{l}\text { Large Underground Xenon } \\
\text { experiment }\end{array}$ & DEAP & $\begin{array}{l}\text { Dark Matter Experiment using } \\
\text { Argon Pulse-shape }\end{array}$ \\
\hline
\end{tabular}




\begin{tabular}{ll|l} 
Symbol & Description & $\underline{\text { Symbol }}$ \\
$\beta$ & $\begin{array}{l}\text { velocity divided by the speed of } \\
\text { light }\end{array}$ & $\Omega_{X}$ \\
$\zeta^{0, r}$ & DM candidate, real part of $\zeta^{0}$ & $\zeta^{Q}$ \\
$H_{k}^{Q}$ & mixed state with charge $Q$ in the & \\
$\mathcal{L}$ & mass basis & $\lambda_{i}$ \\
$\phi$ & Lagrangian density & $\partial_{\mu}, \mathcal{D}_{\mu}$ \\
$\Sigma$ & scalar field & $\Phi$ \\
$\tau^{a}, T^{a}$ & large scalar multiplet & $A^{\mu}, W^{a \mu}$ \\
$X^{*}$ & Complex conjugate of $X$ & {$\left[F_{1} F_{2}\right]_{T}$} \\
$\mathcal{M}$ & matrix element & $X^{\dagger}$ \\
$p_{T}$ & transverse momentum & $\sigma$
\end{tabular}

\section{$\underline{\text { Constant }}$}

$\alpha_{\mathrm{EM}}$

$\alpha_{\mathrm{s}}$

$e$

$g$

$g^{\prime}$

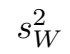

$v$

$c$

$m_{W}$

$m_{Z}$

$m_{h}$

\section{Description}

fine structure constant 1

strong coupling constant

electron charge magnitude

$S U(2)_{L}$ coupling constant

$U(1)_{Y}$ coupling constant

weak mixing angle

vacuum expectation value

speed of light in vacuum 2

mass of the $W$ boson

mass of the $Z$ boson

mass of the Higgs boson

\section{Description}

density parameter for $X$

unmixed multiplet member with

charge $Q$

scalar quartic coupling

partial and covariant derivatives

scalar doublet

gauge fields

total isospin $T$ combination of the

fields $F_{1}$ and $F_{2}$

conjugate transpose of $X$

cross section

missing transverse momentum $\underline{\text { Value }}$

$1 / 128$

0.1185

$\sqrt{4 \pi \alpha_{\mathrm{EM}}} \approx 0.313$

$e / s_{W} \approx 0.651$

$e / c_{W} \approx 0.357$

0.231

$246 \mathrm{GeV}$

1

$80.385 \mathrm{GeV}$

$91.1876 \mathrm{GeV}$

$125 \mathrm{GeV}$

$1_{\text {the value at }} Q^{2}=0$ is $1 / 137$.

${ }^{2}$ in SI units, $c=299792458 \mathrm{~m} / \mathrm{s}$. 


\section{CHAPTER 1}

\section{Introduction}

How do we explore the world around us? We might move closer to a flower to see its petals or smell its scent. We could turn our head towards the barking dog to confirm that it is, indeed, behind a fence and will not chase us. But what about the worlds that we cannot directly observe with our senses?

The experimental particle physicist would respond that we discover information about the invisible world by colliding known sub-atomic particles together and making measurements on the outcome of those collisions. The astrophysicist would tell us to look through telescopes, either those based on the surface of the Earth or those in orbit around it. Using specialized filters and electronics, we can look at a much wider range of wavelengths of light than with just our eyes. The mathematician would advise us to look inward, to use self-consistent systems of logic to deduce relations between numerical quantities. By combining the observations of the experimental physicist with those of the astrophysicist, and using the mathematician's logical system, we are able to create a framework in which to make predictions about future observations.

One of the most successful frameworks that we have built is the Standard Model of particle physics. It describes three of the four known forces of nature. It can be used to explain and predict a wide variety of experimental results in the sub-atomic world. It is not without its faults, however, and one of its missing ingredients is a mysterious substance that we call dark matter, which is responsible for much of the structure we see in our Universe. Despite not knowing what it is, we can describe 
some of its general properties, build mathematical models to attempt to define it, and build experiments that search for it. In this thesis, we present one such model and use experimental results to place constraints on the parameter space of our model.

In this chapter, we will introduce the Standard Model of particle physics and discuss its strengths and weaknesses. We will then describe dark matter, give some potential candidates, and discuss how to go about finding it. Finally, we will introduce the large multiplet models that will be examined throughout this thesis.

\subsection{The Standard Model of particle physics}

The Standard Model (SM) is a framework that describes the particles that make up our Universe and their interactions. It is given, in mathematical terms, by a Lagrangian density and a set of parameters that must be measured by experiment. A culmination of the inductive and reductive enterprise of modern physics, it is one of the most successful scientific theories which we have devised. The precise mathematical structure of the SM will be detailed throughout later chapters of this thesis. For now, we provide a general overview of its constituent parts, experimental successes, and its shortcomings.

We look out at the Universe around us and we see that it contains matter and energy. The matter and energy act on each other through the forces. We experience manifestations of two forces regularly. Gravity is the reason why objects fall towards the centre of the Earth and why the moon orbits our planet. Electromagnetism (EM) gives us light to see, electricity to power our wonderful gadgets, and magnets that overcome the force of gravity to hold a grocery list to the fridge door. It turns out that these are not the only forces of nature. There are two more forces that we don't necessarily notice on the human scale, but are every bit as important. The strong 


\begin{tabular}{ccc}
\hline \hline Force & $\begin{array}{c}\text { Strength Relative to } \\
\text { Electromagnetism }\end{array}$ & Range $[\mathrm{m}]$ \\
\hline Strong Nuclear Force & 1000 & $10^{-15}$ \\
Electromagnetism & 1 & $\infty$ \\
Weak Nuclear Force & $10^{-11}$ & $10^{-17}$ \\
Gravity & $10^{-40}$ & $\infty$ \\
\hline \hline
\end{tabular}

TABLE 1.1. Relative strengths of the fundamental forces. Values are given relative to the electromagnetic force [7].

nuclear force is responsible for binding together nuclei, and the weak nuclear force is responsible for changing the species of a particle. The four forces act with different strengths and over vastly different ranges, summarized in Table 1.1.

Everyday matter - the stars, tigers, and books - that make up our Universe are made from atoms. These are in turn made up of protons and neutrons (confined to a nucleus), and electrons. The electron is, as far as we can tell, a fundamental particle of nature. It experiences electromagnetism and the weak force 1 . The electron is a member of a family of particles called leptons. There are three "flavours" of leptons with charged and neutral versions.

Unlike leptons, the protons and neutrons are composite objects. They are constructed from two types of quarks, fundamental subatomic particles that experience the strong force, the weak force, and electromagnetism. Quarks come in six "flavours", organized into three generations. Taken together, these twelve particles (six leptons

${ }^{1}$ All of the particles of the Standard Model will also experience gravitational forces, but since the gravitational force is proportional to the mass of the interacting objects, it is significantly weaker. For example, the force due to gravity between two protons in a helium nucleus is roughly $F_{\mathrm{G}} \approx 10^{-43} \mathrm{~N}$, while the electromagnetic force is $F_{\mathrm{EM}} \approx 10^{-7} \mathrm{~N}$. 


\begin{tabular}{|c|c|c|c|c|c|}
\hline $2 / 3$ & $1 / 2$ & $2 / 3$ & $1 / 2$ & $2 / 3$ & $1 / 2$ \\
\hline \multicolumn{2}{|c|}{$u$} & \multirow{2}{*}{\multicolumn{2}{|c|}{$\begin{array}{c}c \\
\text { charm }\end{array}$}} & \multirow{3}{*}{\multicolumn{2}{|c|}{$\begin{array}{c}t \\
\text { top } \\
173.2 \mathrm{GeV}\end{array}$}} \\
\hline & & & & & \\
\hline \multicolumn{2}{|c|}{$2.3 \mathrm{MeV}$} & \multicolumn{2}{|c|}{$1.275 \mathrm{GeV}$} & & \\
\hline$-1 / 3$ & $1 / 2$ & $-1 / 3$ & $1 / 2$ & $-1 / 3$ & $1 / 2$ \\
\hline \multirow{3}{*}{\multicolumn{2}{|c|}{$\begin{array}{c}d \\
\text { down } \\
4.8 \mathrm{MeV}\end{array}$}} & \multirow{3}{*}{\multicolumn{2}{|c|}{$\begin{array}{c}s \\
\text { strange } \\
95 \mathrm{MeV}\end{array}$}} & \multirow{3}{*}{\multicolumn{2}{|c|}{$\begin{array}{c}b \\
\text { bottom } \\
4.2 \mathrm{GeV}\end{array}$}} \\
\hline & & & & & \\
\hline & & & & & \\
\hline-1 & $1 / 2$ & -1 & $1 / 2$ & -1 & $1 / 2$ \\
\hline \multirow{3}{*}{\multicolumn{2}{|c|}{$\begin{array}{c}e \\
\text { electron } \\
511 \mathrm{keV}\end{array}$}} & \multirow{3}{*}{\multicolumn{2}{|c|}{$\begin{array}{c}\mu \\
\text { muon } \\
106 \mathrm{MeV}\end{array}$}} & \multirow{3}{*}{\multicolumn{2}{|c|}{$\begin{array}{c}\tau \\
\text { tau } \\
1.78 \mathrm{GeV}\end{array}$}} \\
\hline & & & & & \\
\hline & & & & & \\
\hline 0 & $1 / 2$ & 0 & $1 / 2$ & 0 & $1 / 2$ \\
\hline \multirow{2}{*}{\multicolumn{2}{|c|}{$\begin{array}{c}\nu_{e} \\
\text { electron } \\
\text { neutrino }\end{array}$}} & \multirow{2}{*}{\multicolumn{2}{|c|}{$\begin{array}{c}\nu_{\mu} \\
\text { muon } \\
\text { neutrino }\end{array}$}} & \multicolumn{2}{|c|}{$\nu_{\tau}$} \\
\hline & & & & ne & \\
\hline \multicolumn{2}{|c|}{0} & \multicolumn{2}{|c|}{0} & \multicolumn{2}{|c|}{0} \\
\hline
\end{tabular}

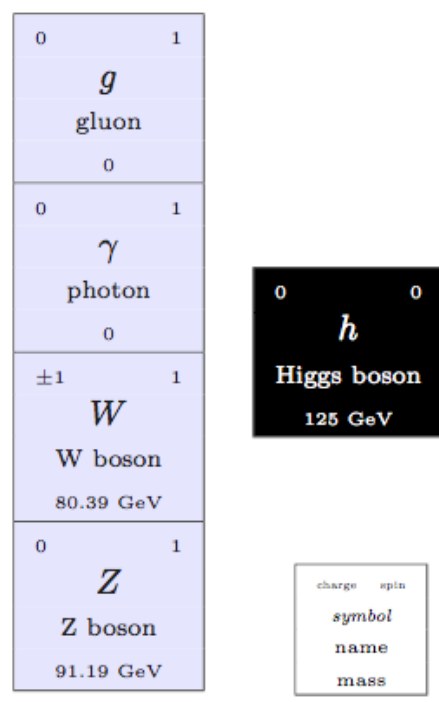

Figure 1.1. Particles that make up the Standard Model. Entries in red are quarks, those in green are leptons, those in blue are gauge bosons, and the black entry is the Higgs boson. The up and down quarks (which make up the protons and neutrons), the electron, and the photon are those particles with which we are most familiar.

and six quarks) interact via four force-carrying particles and one further neutral particle, the Higgs boson. The table in Figure 1.1 shows the particles grouped in quarks (top six red blocks), leptons (bottom six green blocks), and force-carriers (four blue blocks).

Of these, only four are commonly encountered in our Universe: $u$ (up quark), $d$ (down quark), $e$ (electron), and $\gamma$ (photon). The electron and photon are familiar, while the proton and neutron are made up of three quarks each, uud and udd respectively. Just as more experimentation filled in the periodic table of elements, further experiments in particle physics revealed the existence of greater numbers of fundamental particles. These particles have similar quantum mechanical properties as the $u, d, e$, and $\gamma$, but differ in mass from their counterparts. 
The particles that describe the forces come in charged and uncharged varieties, as well as in massive and massless varieties. The three forces that they mediate are electromagnetism $(\gamma)$, the weak nuclear force $\left(W^{ \pm}\right.$and $\left.Z\right)$, and the strong nuclear force $(g)$. The suggestive grouping and classifying of the particles in Figure 1.1 come from how they respond to the three forces, as well as certain properties they exhibit. The number in the top right corner of each entry is the particle's spin. The quarks and leptons are all spin-1/2 (fermions), while the force-carriers (also called as gauge bosons) are spin-1, and the Higgs (a scalar boson) is spin-0. The number given in the top left corner of each entry is its electric charge. In each of the quark and lepton tables, the mass increases from left to right and the electric charge for the top rows is higher than for the lower rows. Every particle with a non-zero electric charge participates in the electromagnetic interactions, and so may absorb or emit photons without changing flavour. Neutral weak interactions proceed via $Z$ bosons and occur between any two quarks or leptons of the same species. Charged weak interactions change the particle flavour within the same generation. That is, an electron may become an electron neutrino by emitting a $W^{-}$(written as $e^{-} \rightarrow W^{-} \nu_{e}$ ), or a downtype quark will become an up-type quark via the absorption of a $W^{+}$boson (written as $\left.d W^{+} \rightarrow u\right)$.

Particles that experience the strong force - those that interact via gluons, as described by Quantum Chromodynamics (QCD) - are the quarks. In addition to the flavours, quarks carry an additional charge called colour. Quarks may carry any one of three colours, while gluons carry a colour-anti-colour pair. The colour of a quark may be changed by an interaction with a gluon. Objects we observe in nature are colourless which dictates the constituent particles of these objects: objects that contain either a colour-anti-colour pair or three colours (or anti-colours). An object 
with a quark of one colour and an antiquark with the corresponding anti-colour is called a meson. Those with three quarks, one of each colour, are called baryons. While there have been some tantalizing experimental hints of other combinations of quarks (e.g., a four-quark state [8] and five-quark state [9], though these may be temporary bound states of two mesons or a meson and a baryon respectively), the SM predicts only two- and three-quark combinations, collectively called hadrons.

The final piece of the table of fundamental particles is a somewhat special particle. The Higgs boson is a consequence of the spontaneous symmetry breaking of the electroweak (EW) sector to the electromagnetic sector. Its existence provides evidence of the ABEGHHK'tH'2 (or, more commonly, Higgs) mechanism, which describes how some particles in the SM acquire mass. The vacuum of our Universe is not truly empty: the Higgs field exists as a non-zero energy field from which a Higgs boson can be excited. The interactions with the Higgs field provides the SM particles with their masses. This final experimentally-observed piece of the SM, the Higgs boson, was found at the CERN Large Hadron Collider (LHC) in 2012 [10, 11].

The Higgs is not the only successful prediction of the SM, only the most recent. Figure 1.2 displays the agreement of the SM predictions with experimental observations. The left-hand plot shows the absolute difference between measurements of masses and particle widths (the width of a particle is the inverse of its lifetime) from CERN's Large Electron-Positron Collider (LEP) and values predicted from a fit of the SM, normalized to their experimental uncertainty. All of the values shown here give remarkable agreement; values of zero would indicate perfect agreement, but in each observable, the difference between theory and experiment is less than the experimental uncertainty. In the right-hand plot, the ATLAS (A Toroidal LHC ApparatuS)

${ }^{2}$ for Philip Warren Anderson, Robert Brout, François Englert, Gerald Guralnik, C. R. Hagen, Peter Higgs, Tom Kibble, and Gerard 't Hooft. 

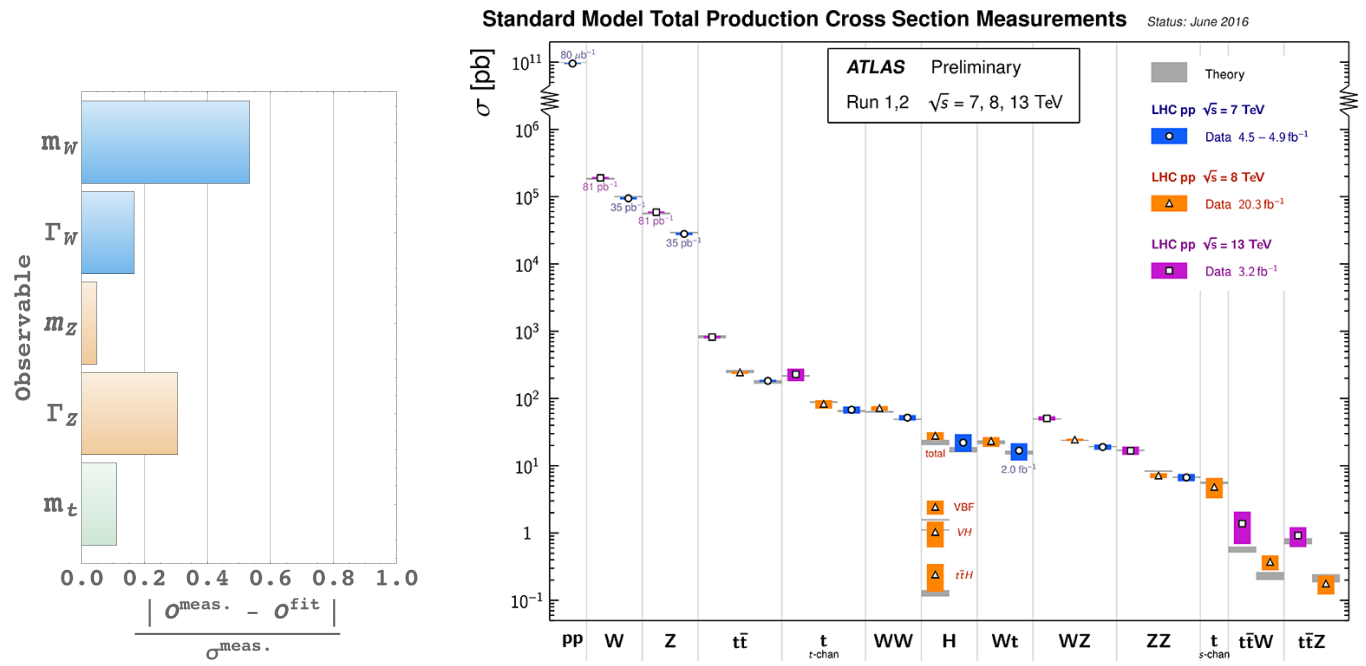

FiguRE 1.2. SM predictions compared with experimental measurements. The plot on the left shows a comparison between values predicted by the SM and measurements made at LEP for various physical quantities of the electroweak part of the SM. The plot on the right shows the comparison between SM predictions and measurements made by the ATLAS detector at the LHC for a wide variety of SM interaction cross sections. Left-hand plot uses data from Table F.1 of Ref. [15]; righthand plot courtesy the ATLAS Standard Model physics group [16].

experiment at the LHC gives a comparison between the predicted interaction cross section and the measured value for a large number of SM processes. The agreement in this plot is impressive in that the values being compared agree over many orders of magnitude and at different energies ${ }^{3}$. The details of the SM are reviewed in, e.g., Refs. [6, 12, 13, 14].

Despite the triumph of the SM in explaining experimental results, there exist cracks in its façade. The most glaring problem is that the SM lacks the fourth force: gravity. Our current understanding of gravity is through General Relativity (GR) [17], which describes gravity as the curvature of four-dimensional spacetime.

\footnotetext{
${ }^{3}$ The LHC ran at a centre-of-mass energy of $\sqrt{s}=7 \mathrm{TeV}$ in $2010, \sqrt{s}=8 \mathrm{TeV}$ in 2012, and $\sqrt{s}=13 \mathrm{TeV}$ in $2015-2016$.
} 
While it is not entirely incompatible with the SM, there is no way to get from one theory to the other. Worse, in situations where both theories must apply (e.g., black holes: sources of strong gravitational fields at arbitrarily small distances), the discrete nature of quantum theory and the smoothness of GR conflict disastrously. Furthermore, the force due to gravity is far weaker than any of the other three forces (see Table 1.1). The lack of any clear reason why this should be the case is called the hierarchy problem.

Beyond this, the SM itself gives rise to some confounding results: why are there three generations of fermions, and why are their masses arranged in the way they are? The oscillation of neutrinos indicates that they must have mass, but the SM predicts they are massless; what is the origin of this mass? Why does the Universe contain baryons, but not anti-baryons in large quantities? Astronomical observations require some form of cold dark matter (DM) that does not appear in the SM; what is its nature?

Although each of these questions is interesting, it is this last which concerns the remainder of this thesis. We seek a model that contains a viable DM candidate; that is, a model for DM that staisfies current experimental constraints, and provides observable experimental signatures.

\subsection{Dark matter}

The experimental evidence that some form of cold dark matter exists began with observations by Jan Oort [18] and Fritz Zwicky [19, 20] in the 1930s. Oort was examining the velocities of stars nearby in our own galaxy, while Zwicky was studying the Coma cluster of galaxies. Based on measurements of the light output and assumptions about the less luminous matter that would be expected to be there, they 


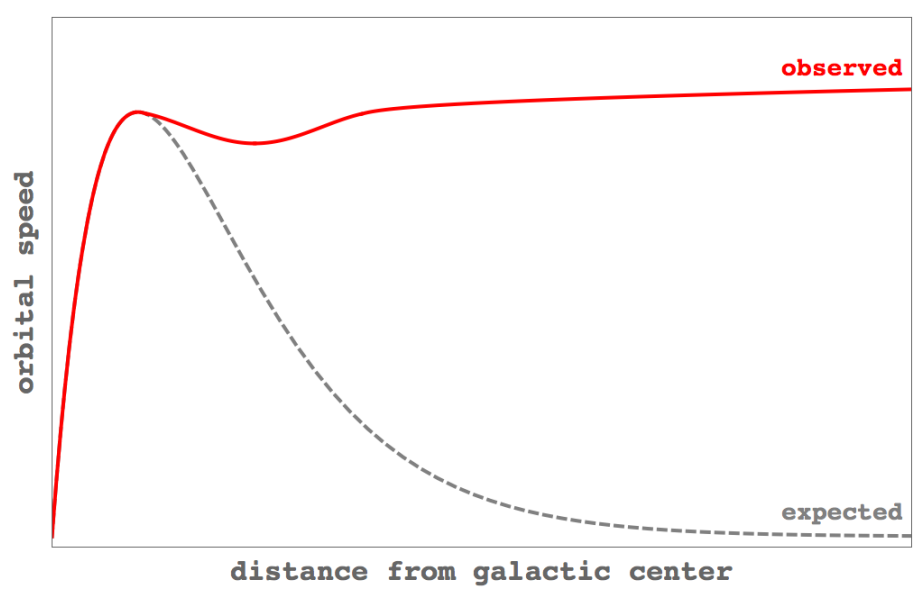

FIGURE 1.3. Sketch of a galactic rotation curve showing the requirement for some form of dark matter. The expected curve shows the Keplerian fall-off with distance, while the observations show that the speed remains nearly constant as the distance from the galactic centre increases.

both found that the orbital speeds were far too high. From Newtonian dynamics, as the distance from the centre of a galaxy (or centre-of-mass of a cluster) increases, the orbital velocity should fall off as seen in the dashed grey curve of Figure 1.3. However, the measured velocity was not decreasing with distance, but remained nearly constant out to a large distance (the red curve in Figure 1.3). For this to occur, the assumed gravitational effects were not properly accounted for: either gravity as we knew it was wrong, or the amount of matter we infer in these locations is wrong. The observations were largely ignored until the 1970s when Vera Rubin made much more precise determinations of a large number of galactic rotation curves [21, 22]. With the updated instrumentation and measurements of the matter content of typical galaxies, she showed that as much as $50 \%$ of the mass of a galaxy must be contained in a non-luminous halo. 


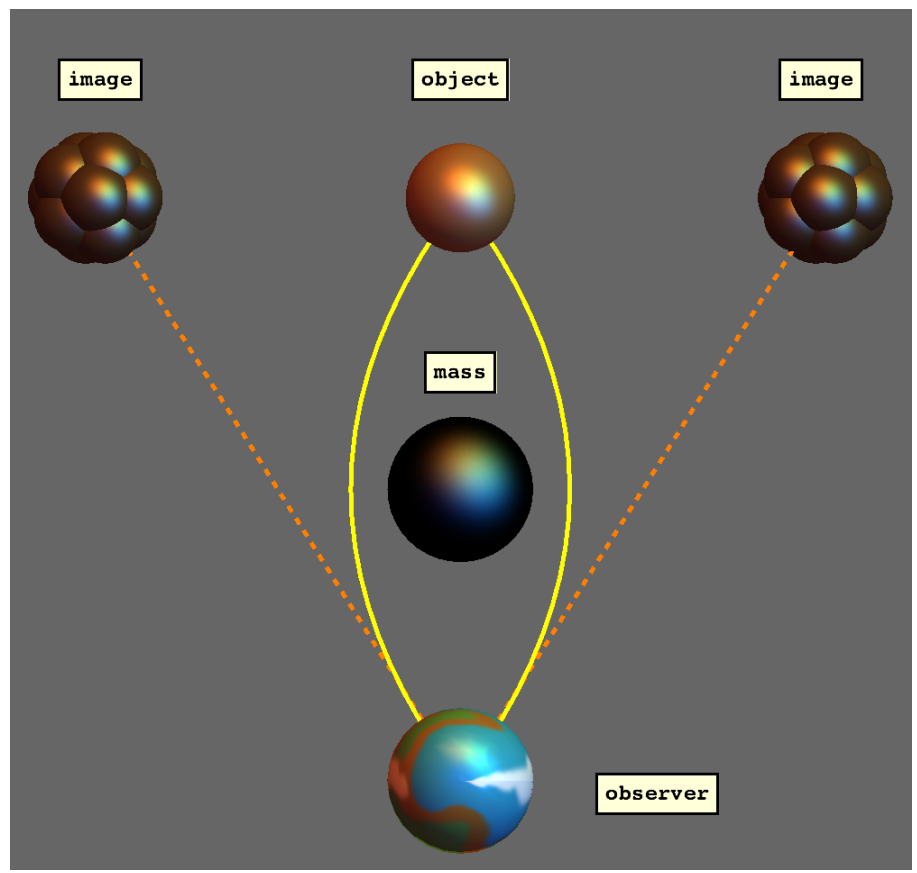

FiguRE 1.4. Gravitational lensing of a distant object by an intervening mass. The mass between the distant object and the observer distorts spacetime and causes diverging light rays from the distant object to instead converge at the observer. The observer then sees two (distorted) images of the distant object at different positions in the sky.

Although its existence was first discovered in rotation curves, there are many other routes that all end in some form of DM. Massive objects affect the path of light. In everyday experience, this effect is far too small to be seen, but for very massive objects, the effect will be quite pronounced. This effect was first predicted by Einstein [23], and measured by Arthur Eddington during a solar eclipse in 1921 [24]. In astrophysical situations, this may be seen when observing a distant object with a galaxy in the line of sight, as in Figure 1.4. The mass situated between the observer and the object will curve the path of light leaving the object. This causes (distorted) images to be seen by the observer. This gravitational lensing has been observed in 
many situations to the extent that, again, the visible matter present in the intervening galaxy cannot produce the magnitude of the lensing effect observed.

There is a standard cosmological model $(\Lambda$-CDM, where $\Lambda$ is the usual cosmological constant and CDM stands for Cold Dark Matter) that describes how the Universe formed from the Big Bang predicted by GR. One of the expected outputs of this model is the formation of structure in the Universe, such as planets, stars, galaxies, clusters, and filaments (clusters of clusters). Based on when structures formed in the early Universe, there must be some form of DM. Otherwise, radiation pressure will slow down the gravitational collapse of ordinary matter, and structure would form much later. Furthermore, the timing of structure formation and the "clumpiness" of the structure rules out one form of possible DM from the SM: neutrinos have mass, are electrically neutral, very weakly interacting, and stable, but their masses are so small that they have been relativistic until quite recently in the history of the Universe. That they are relativistic would wash out nearly any density fluctuations in the early Universe.

Very early on in the thermal history of the Universe, the mean-free path of photons was short - the highly ionized plasma was continually emitting and absorbing photons. This meant that the early Universe was opaque. As the plasma cooled, the protons were able to capture electrons, forming electrically neutral atoms. These neutral atoms no longer emitted nor absorbed photons, which were now able to travel unimpeded. This last flash of light gives a snapshot of the Universe as it was three hundred and eighty thousand years after the Big Bang. As time went on, this light redshifted from the high-energy gamma rays which it began life to the microwave region. Satellites have mapped this Cosmic Microwave Background Radiation, first 


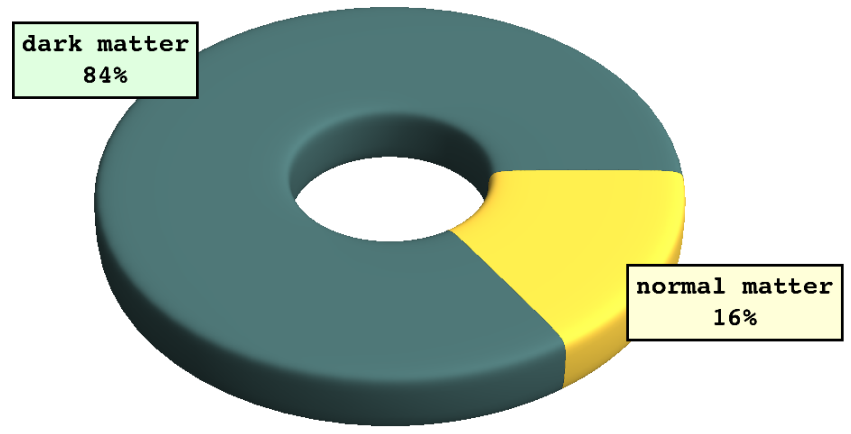

FiguRE 1.5. Matter content of the Universe. Based on cosmological observations, the Universe should contain roughly $16 \%$ of SM particles by mass and $84 \%$ of DM particles. Figure uses data from Table 4 of Ref. [25].

discovered by Penzias and Wilson in 1965, and found minute variations in the temperature which would correspond indirectly to structures in today's Universe. Further measurements (from, e.g., the Planck experiment [25]) indicate that the amount of dark matter is roughly five times greater by mass than the amount of SM particles in today's Universe (see Figure 1.5).

We see from many different observations that DM is required, and indeed exists. However, we know very little about its nature. We know that (1) it must be stable (at least on cosmological timescales); (2) it is electrically neutral; and (3) it interacts gravitationally. This might lead us to think that our understanding of gravity is incorrect. Attempts to modify gravity such as Modified Newtonian Dynamics (MOND) [26] or Tensor-Scalar-Vector (TeVeS) [27] models work on small scales but have difficulty in explaining cosmological observations [28]. Massive Compact Halo Objects (MACHOs) are non-luminous (or at least of sufficiently low luminosity) bodies that are present in galaxies that could cause the discrepancy in galactic rotation curves. Due to microlensing (gravitational lensing by planet- or stellar-sized objects) 
experiments, there are strong constraints on the number of these MACHOs [29, $\mathbf{3 0}$. They would also be unable to account for cosmological observations.

An explanation of DM in terms of some new particle is much more appealing. Unfortunately, no such particle has been detected to date. Some of the more promising candidates include axions [31, 32, 33], asymmetric DM [34, 35, 36], DM from extra dimensions [37, 38, 39, 40], hidden sector DM [41, 42], and Weakly Interacting Massive Particles (WIMPs) [43, 44, 45].

Axions come about as a solutions to the strong CP (charge-parity) problem in the SM [46, 47]. The vacuum structure of QCD induces an extra term in the SM Lagrangian which produces an electric dipole moment of the neutron. The bounds on this electric dipole moment are vanishingly small, while the naïve expectation from the SM is of order one. The resolution of this apparent discrepancy is to introduce an extra symmetry of the SM Lagrangian. This symmetry is spontaneously broken and dynamically suppresses the CP-violating term. The Goldstone boson of this broken symmetry is the axion. The energy scale of the symmetry breaking that produces the axion must be high, based on experimental measurements, which sets the mass of the axion to be very light ( $\mu \mathrm{eV}$ to meV [6]).

Asymmetric DM is a class of models that presume that the mechanism that caused the asymmetry between baryons and anti-baryons in the Universe also caused an asymmetry between DM and its antiparticle [48]. Such models add an extra symmetry to the SM under which the SM particles are uncharged, while the DM and anti-DM have opposite charges. Then, the DM and anti-DM will annihilate each other until only the DM remains, matching the present-day density of DM. Since in these models the ratio of the relic abundance of DM to baryons, $\Omega_{\mathrm{DM}} / \Omega_{\mathrm{b}} \sim 5 \sim m_{\mathrm{DM}} / m_{p}$, where $m_{p}$ is the proton's mass, the mass of the DM is of order $1-10 \mathrm{GeV}$. 
We know that gravity is much weaker than the other three forces. One possible way of explaining this discrepancy is for the particle that mediates gravity to travel in an additional spatial dimension [49, 50, 51]. Such extra-dimensional models predict excitations into this extra dimension of SM particles which could be natural DM candidates.

It is possible that there are are, hidden, a vast number of non-SM particles that interact with the SM through a very small number of mediator particles [52, 53]. One such example would be Mirror Dark Matter [54], where every SM particle has a counterpart in a separate sector that only interacts with the SM via mixing between the photons of both sectors. In that case, the mirror particles could collectively constitute "hidden sector" dark matter.

Finally, the most promising (and most popular) DM candidate is the Weakly Interacting Massive Particle. These particles would be produced in the early Universe and freeze-out in a similar manner to SM neutrinos (the nature of freeze-out is of some importance and will be discussed in detail in Section 6.2. For a WIMP with a mass in the $\mathrm{GeV}-\mathrm{TeV}$ range and $\mathrm{SM}$ interactions on the same order as the weak force, the relic abundance of thermal WIMPs matches that required for cold dark matter. This is known as the WIMP miracle. WIMPs may arise in many models such as Supersymmetry [55, Minimal Dark Matter [56, 57, 58, and little Higgs [59, 60, 61]. The WIMP paradigm is currently the default lens through which to search for DM.

\subsection{Experimental pathways to the observation of dark matter}

We have seen that DM must exist and briefly described some appealing candidates. Now we ask the question of how to observe DM. Using gravitational lensing maps, we are able to show regions where DM should be. This only tells us where it is, not 


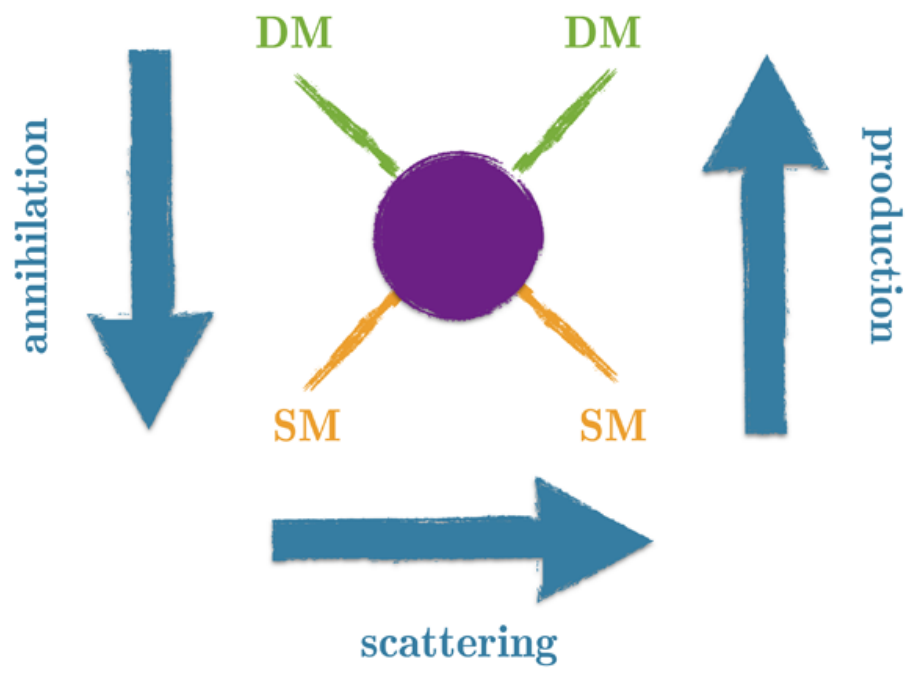

Figure 1.6. Possible DM-SM interactions. Each arrow represents a flow of time. Annihilation of two DM particles into two SM particles follows the down arrow. Scattering of a DM particle off of a SM particle follows the horizontal arrow. Annihilation of two SM particles into two DM particles follows the up arrow. The blob at the centre of the diagram may be a very complicated process. The horizontal arrow may be probed by direct detection experiments, the up arrow by collider experiments, and the down arrow by indirect means.

what it is. To do that, we need to perform experiments here on Earth. If the DM is a WIMP, then it may interact with the SM in ways beyond just gravitationally. Schematically, these types of interactions are shown in Figure 1.6. Each of the three arrows represents a flow of time in the diagram and corresponds to a possible avenue of experimental detection:

- indirect detection: when two DM particles annihilate into two SM particles;

- direct detection: when a DM particle scatters off of a SM particle; and

- particle colliders: used to create DM through the annihilation of SM particles.

The direct detection experiments are the most straightforward approach to observing WIMP DM (the horizontal arrow in Figure 1.6). First, gather together a large volume of detector material in a background-free environment and then wait to see a 
rare scattering event $[\mathbf{6 2}, 6 \mathbf{6 3}]$. The question then becomes what sort of detector material to use. The most efficient way of observing WIMP DM in this manner is through the elastic scattering of a DM particle off a target atomic nucleus. These experiments want a heavy stable radio-pure element. Experiments such as Cryogenic Dark Matter Search (CDMS) [64], EDELWEISS [65, and Coherent Germanium Neutrino Technology (CoGeNT) [66] use solid-state germanium detectors to search for low-mass WIMP DM $\left(m_{\mathrm{DM}} \lesssim 10 \mathrm{GeV}\right)$. For heavier WIMPs $\left(m_{\mathrm{DM}} \sim 50-1000 \mathrm{GeV}\right)$, largescale experiments such as the Large Underground Xenon dark matter experiment (LUX) [67], XENON1T [68], and Dark matter Experiment using Argon Pulse-shape discrimination (DEAP-3600) [69] use liquid noble gases (xenon, xenon, and argon respectively). The liquid noble gas experiments will be more applicable to the models we will examine, and so we will give a more detailed description of how they function in Section 6.5.

If the WIMP DM annihilates to SM particles in the present day (the down arrow in Figure 1.6, then signals from those annihilations may be seen in astrophysical observations [70]. The SM particles that would be produced in DM annihilation are high-energy photons, neutrinos, and cosmic rays. Experiments to observe cosmic rays such as PAMELA [71] and AMS-02 [72] have seen an excess in the number of positrons, although this may be due to nearby pulsars rather than DM. If DM is captured by the sun, then it could produce an observable neutrino flux signal in experiments such as IceCube [73]. The most tantalizing indirect observations currently come from the Fermi-LAT telescope [74]. An excess of high-energy gamma rays near the galactic centre suggests that DM is annihilating there [75, 76, 77].

The third (up) arrow in Figure 1.6 corresponds to the production of DM, especially through collider experiments such as LEP [78] and the LHC [79, 80]. To look for 
DM at a collider, we must find energy we cannot see. We know that energy and momentum must be conserved, and so we look for signals where they do not appear to be conserved (momentum of all visible signals is unbalanced) and infer the existence of DM [81, 82].

Related to both the collider and indirect searches, if the DM interacts with the SM in some way, then it may affect SM processes at the loop-level in Feynman diagrams (i.e., at a higher order in perturbation theory). The precise measurements of SM processes leads to strong constraints on the parameter space of DM models.

\subsection{Large electroweak scalar multiplets}

While the models that attempt to solve more than one of the problems of the SM are attractive, they can often be complicated to work with. Simplified models, such as Minimal Dark Matter, seek to solve the DM problem in isolation and with as few additional parameters as possible. The original Minimal Dark Matter model extends the SM by the addition of a single multiplet of $S U(2)_{L}$, their most promising being a fermionic quintuplet, with a DM candidate at a mass of $4.4 \mathrm{TeV}[\mathbf{5 6}, \mathbf{5 7}, \mathbf{5 8}, \mathbf{8 3}$. This quintuplet interacts with the SM only through $S U(2)_{L}$ gauge interactions (the quintuplet's hypercharge is zero), and mass splitting between the states is induced at the loop level. The only parameter of Minimal Dark Matter models is the DM candidate's mass. If we relax the assumption of minimality ever so slightly (a next-to-Minimal Dark Matter, say), we open up a large class of models that have interesting properties and phenomenology. Models of this type have been examined in Refs. [84, 85], which have zero hypercharge and, in addition to the gauge interactions, interactions with the Higgs. Due to couplings to the Higgs, a range of possible DM candidate mass values are allowed. 

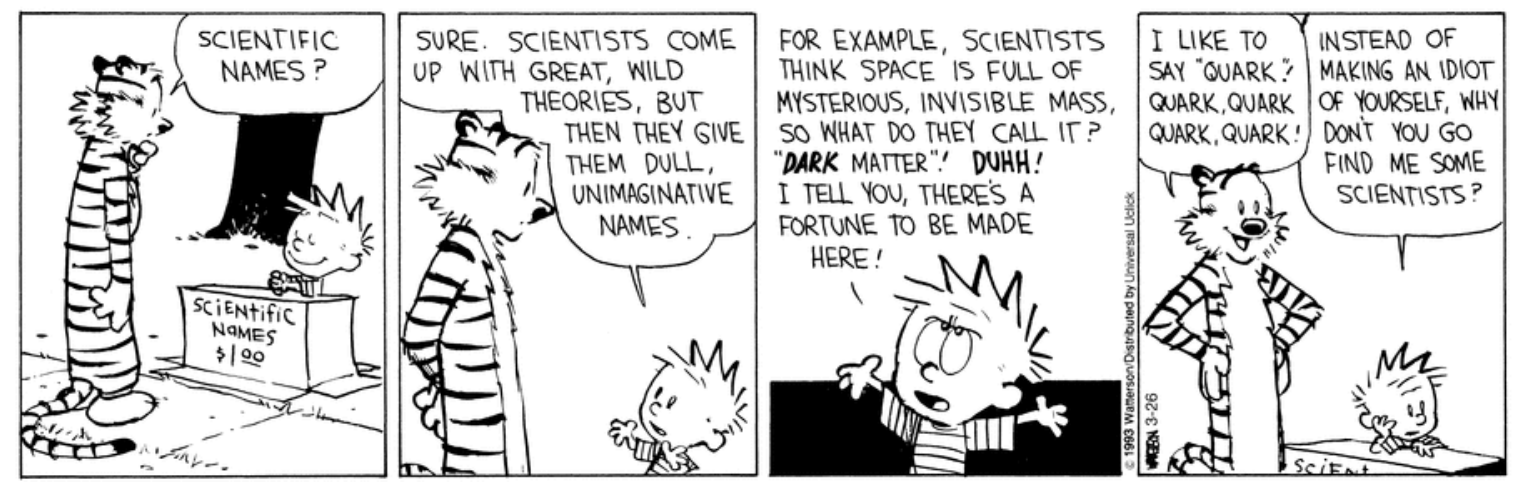

FiguRE 1.7. While the name "dark matter" may seem uncreative, it is very descriptive in that DM does not emit light (it is dark), but it does interact gravitationally (it acts as matter). Similarly, calling our models "large electroweak scalar multiplets" provides a complete description of the model in its name. Calvin And HobBes (c) 1993 Watterson. Reprinted with permission of Universal UClick. All rights reserved.

In the following chapters, we examine a class of these nearly-Minimal DM models that extend the SM by a single large electroweak scalar multiplet. In these models, our interest is to explore the available parameter space and determine the constraints induced by current experimental results. From this, we find viable DM candidates and describe their properties. For DM candidate masses less than a few hundred GeV, we find that collider measurements of SM processes constrain our DM candidate to be only a (small) fraction of the total DM content of the Universe. In the multi-TeV DM mass range, we find that our DM candidate can indeed make up all of the DM content of the Universe, and we determine its required properties using co-annihilations and Sommerfeld enhancement.

This thesis is organized as follows. In Chapter 2, we describe what we mean by "large" electroweak scalar multiplets and give some generic constraints on these types of models. Chapter 3 describes the models of interest in detail, including different parameterizations, mass spectra, and decay branching ratios. We provide theoretical 
constraints in Chapter 4 and discuss the running of the coupling parameters. In Chapter 5, we examine the effects of large electroweak scalar multiplets on SM processes, and use direct searches for new physics (NP) to obtain constraints on the parameter space. Chapter 6 examines the DM constraints, both theoretical and experimental, on the two DM-candidate mass regions. Finally, in Chapter 7, we summarize our results. In the appendices, we provide the full list of Feynman rules for the large multiplet models Appendix A, the properly normalized total isospin combinations of two multiplets (Appendix B), the generators of $S U(2)$ Appendix C), the one-loop renormalization group equations for the scalar quartic couplings and mass parameters Appendix D, and large multiplet models with a $U(1)$-symmetric Lagrangian Appendix E. References are provided starting on page 228. 


\section{CHAPTER 2}

\section{Large electroweak scalar multiplets}

How do we describe the Standard Model (SM)? As in the Introduction, we can set out the known particle content and list the forces that act on each of these particles. To make any sort of prediction using the SM, however, we need to detail its mathematical structure. The SM is described by a Lagrangian, an expression that encodes the particle content and interactions, and which obeys a principle of least action. The mathematics used to describe the SM is the language of group theory. If we want to extend the SM in such a way as to include a dark matter (DM) candidate, we would do well to understand this mathematical structure in order to provide a suitable candidate.

The models described in Section 1.2 all work within the rules set out in group theory, and the large electroweak scalar multiplet models we introduce in Section 1.4 are no different. In this chapter, we first define the group structure of the SM. Next, we describe how to extend the electroweak sector to allow for the inclusion of a DM candidate in the form of electroweak multiplets. We then define large electroweak scalar multiplets. Finally, we use the scattering of multiplet members to SM gauge bosons to place limits on the hypercharge and size of the large multiplets. Throughout the remainder of this thesis, we will use the terms "large electroweak scalar multiplet", "multiplet", and "large multiplet" interchangeably. 


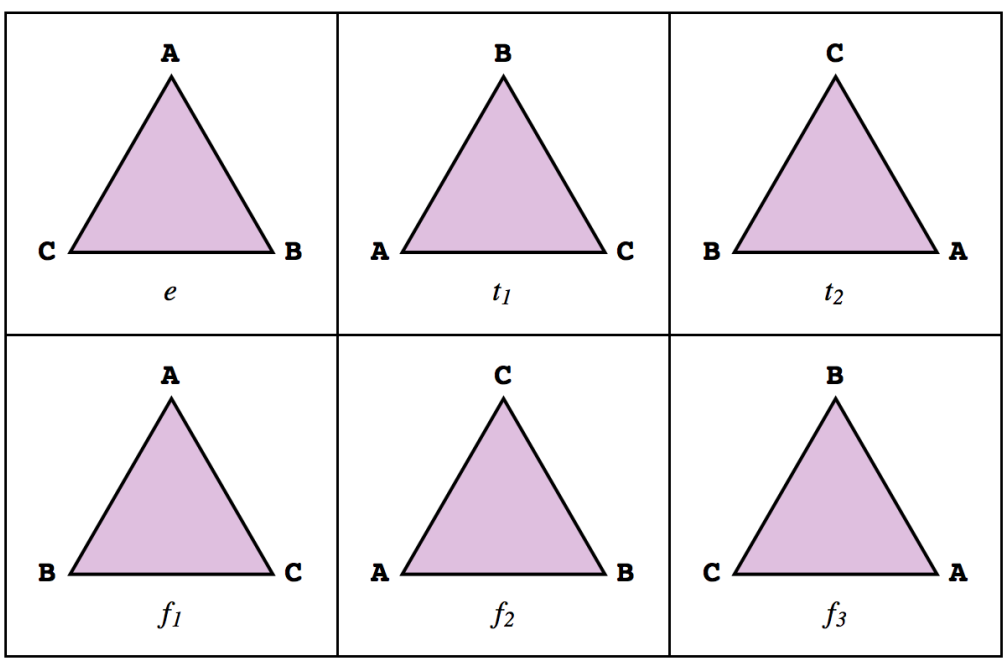

FIGURE 2.1. Rotations and reflections of an equilateral triangle. Each figure is labelled with the accompanying action: $e$ is the identity; $t_{1}$ is rotation by $120^{\circ} ; t_{2}$ is rotation by $240^{\circ} ; f_{1}$ is a reflection about $A ; f_{2}$ is a reflection about $B ; f_{3}$ is a reflection about $C$.

\subsection{Groups and the Standard Model}

Symmetry is a trait desired by humankind in areas of art, architecture, and decorating. We see symmetry in nature in such places as the regular arrangement of honeycombs and the structure of crystal lattices. It is the repetition of a pattern that is symmetry. Mathematically, we say that a symmetry of a system (e.g., a system of equations, a geometric object, a set of numbers) is an operation that leaves the system unchanged.

Consider an equilateral triangle and the actions of counterclockwise rotations and reflections as in Figure 2.1. There are three rotations $\left(0^{\circ}, 120^{\circ}\right.$, and $\left.240^{\circ}\right)$ and three reflections (about a line through the origin and $A, B$, and $C$ respectively). If we ignore the labelling of the corners, the each operation in the figure would leave the resulting figure indistinguishable from the original. The operations collectively form what is called a group. 


\begin{tabular}{c|cccccc}
\hline \hline & $e$ & $t_{1}$ & $t_{2}$ & $f_{1}$ & $f_{2}$ & $f_{3}$ \\
\hline$e$ & $e$ & $t_{1}$ & $t_{2}$ & $f_{1}$ & $f_{2}$ & $f_{3}$ \\
$t_{1}$ & $t_{1}$ & $t_{2}$ & $e$ & $f_{3}$ & $f_{1}$ & $f_{2}$ \\
$t_{2}$ & $t_{2}$ & $e$ & $t_{1}$ & $f_{2}$ & $f_{3}$ & $f_{1}$ \\
$f_{1}$ & $f_{1}$ & $f_{2}$ & $f_{3}$ & $e$ & $t_{1}$ & $t_{2}$ \\
$f_{2}$ & $f_{2}$ & $f_{3}$ & $f_{2}$ & $t_{2}$ & $e$ & $t_{1}$ \\
$f_{3}$ & $f_{3}$ & $f_{1}$ & $f_{1}$ & $t_{1}$ & $t_{2}$ & $e$ \\
\hline \hline
\end{tabular}

TABLE 2.1. Multiplication table for the triangle group. The labels are as in Figure 2.1: $e$ is the identity; $t_{1}$ is rotation by $120^{\circ} ; t_{2}$ is rotation by $240^{\circ} ; f_{1}$ is a reflection about $A ; f_{2}$ is a reflection about $B ; f_{3}$ is a reflection about $C$. In this table, the action on the vertical is performed first and then the action along the top. That is, $\left(t_{2} \circ t_{1}\right) \triangle=e \triangle$.

A group is a set and an operation that acts on pairs of elements from the set and returns an element from the set [86]. A set, $\mathcal{G}$, and and operation, o, must satisfy the following to be a group:

- if $f, g \in \mathcal{G}$, then $f \circ g=h \in \mathcal{G}$ (the set is closed under the operation);

- for $f, g, h \in \mathcal{G},(f \circ g) \circ h=f \circ(g \circ h)$ (the operation is associative);

- there is an element $e \in \mathcal{G}$ such that for all $f \in \mathcal{G}, e \circ f=f \circ e=f(e$ is an identity);

- every element, $f \in \mathcal{G}$, has an inverse, $f^{-1} \in \mathcal{G}$, such that $f \circ f^{-1}=e=f^{-1} \circ f$. In the example of the triangle group above, the elements of $\mathcal{G}$ are the rotations and reflections, and the operation is composition (e.g., do a rotation of $120^{\circ}$ and then a reflection about $C$, which would be written as $\left.\left(f_{3} \circ t_{1}\right) \triangle=f_{2} \triangle\right)$. We can describe the result of each operation in a multiplication table, shown in Table 2.1. 
The triangle group is an example of a finite group. Groups can also have an infinite number of elements, as in the rotations of a sphere, or in the addition of integers. For the last case, the operation is addition, the identity is 0 , and the inverse of integer $n$ is $-n$.

An important class of groups is the Lie groups, where the set $\mathcal{G}$ is a smooth manifold and the operations are differentiable. That is, a Lie group is a set of points in Euclidean space such that all points within a spherical neighbourhood of an arbitrary point can be located by a set of curvilinear coordinates [87]. In addition, wherever two coordinate patches overlap, there is an invertible mapping from one set of coordinates to the other. Perhaps the most important type of Lie groups is $G L(n, \mathbb{C})$, the group of all non-singular $n \times n$ matrices with complex entries. Subgroups of $G L(n, \mathbb{C})$ that are of interest in particle physics are $U(n)$, the $n \times n$ complex matrices with determinant \pm 1 , and $S U(n)$, the $n \times n$ complex matrices with determinant +1 . The operation of $G L(n, \mathbb{C})$ and its subgroups is the usual matrix multiplication.

A physical theory such as the SM may be given by an action, $\mathcal{S}$, which is a functional of the fields of the theory, $\mathcal{S} \equiv \mathcal{S}\left[\phi_{i}, \partial_{\mu} \phi_{i}\right]$. Further, the action may be written as an integral of a Lagrangian (density), $\mathcal{L}$, such that

$$
\mathcal{S}\left[\phi_{i}, \partial_{\mu} \phi_{i}\right]=\int d^{4} x \mathcal{L}\left[\phi_{i}(x), \partial_{\mu} \phi_{i}(x)\right]
$$

For a group, $\mathcal{G}$, with elements $g \in \mathcal{G}$, we call $\mathcal{G}$ a global symmetry of an action if $\mathcal{S}\left[g \phi_{i}, g \partial_{\mu} \phi_{i}\right]=\mathcal{S}\left[\phi_{i}, \partial_{\mu} \phi_{i}\right]$. If the action is invariant under $\mathcal{G}$ up to surface terms, $\mathcal{S}\left[g \phi_{i}, g \partial_{\mu} \phi_{i}\right]=\mathcal{S}\left[\phi_{i}, \partial_{\mu} \phi_{i}\right]+$ surface terms, then we call it a gauge group. If, in addition, the transformations of $\mathcal{G}$ are time-dependent, then the theory is called a gauge theory [88]. 
To study the global structure of a Lie group, it can often be simpler to study its local structure. Consider a neighbourhood, $\mathcal{U}_{g} \subset \mathcal{G}$, around a point $g \in \mathcal{G}$. Then the set obtained by acting on the neighbourhood with the inverse of $g, g^{-1} \circ \mathcal{U}_{g}$, will contain a coordinate patch around the identity of $\mathcal{G}$. Since $g$ is arbitrary, this means that every coordinate patch may be pulled back to the identity [87. We then consider the tangent space to the identity with multiplication

$$
[f, g]=-[g, f]
$$

satisfying

$$
[[f, g], h]+[[g, h], f]+[[h, f], g]=0 \text {. }
$$

This tangent space and multiplication make up the Lie algebra of $\mathcal{G}$, denoted $\mathfrak{g}$. As an example, consider the vector cross-product in three dimensions. Then $\mathfrak{g}=\mathbb{R}^{3}$ and $[\vec{f}, \vec{g}]=\vec{f} \times \vec{g}$. The algebra of $G L(n, \mathbb{C})$ is given by $\mathfrak{g l}$, such that $[A, B]=A B-B A$ is the usual matrix multiplication. We can recover the local structure of $\mathcal{G}$ from $\mathfrak{g}$ in the following manner. First, there exists a mapping exp $: \mathfrak{g} \rightarrow \mathcal{G}$ such that

$$
\exp (A)=\mathbb{1}+A+\frac{1}{2} A A+\frac{1}{6} A A A+\cdots
$$

for matrices $A \in \mathfrak{g}$ and $\exp (A) \in \mathcal{G}$. Then we can construct the local structure of $\mathcal{G}$ by performing this exponentiation for every member of $\mathfrak{g}$.

As is the case for vector spaces, we may often construct a group with infinite elements from a finite subset of those elements, the vector space's basis. For a group, we call this subset the generators of the group. For a group such as $U(n)$, there are $n^{2}$ generators, while for $S U(n)$ there are $n^{2}-1$ generators. Using the exponential 
map from above, we can write the elements of $\mathcal{G}$ as

$$
\exp \left(i \theta_{a} T^{a}\right)
$$

where $a=1, \ldots, N$ runs over the number of generators, $\theta_{a}$ are the parameters, and $T^{a}$ are the generators which satisfy Equation (2.2) as

$$
\left[T^{a}, T^{b}\right]=i f^{a b c} T^{c}
$$

In the above, $f^{a b c}$ are the totally anti-symmetric structure constants of the group. From elementary quantum mechanics, we know that spin is described by $S U(2)$. We call particles with half-odd integer spin fermions and particles with integer spin bosons. Particles with spin $S$ may have eigenvalue of one component of the spin vector of $-S,-S+1, \ldots, S-1, S$. Thus, an electron (fermion with $S=1 / 2$ ) may be either spin-up, $+1 / 2$, or spin-down, $-1 / 2$. A $W$ boson (vector boson with $S=1$ ) may have three spin (polarization) states: $-1,0,+1$. A similar quantity called isospin is also described by $S U(2)$ and will be discussed in Section 2.2. To say why we want isospin, we must first define Noether's theorem and conserved quantities.

One of the most important results of modern physics is Noether's theorem [89, $\mathbf{9 0}$, which states that for every symmetry of a system, there exists a conserved quantity (such as electric charge or energy). For example, the equations of Newton's mechanics are invariant under spatial translations and rotations (the Euclidean group), leading to conservation of linear and angular momentum, respectively. They are also invariant to time translations, leading to conservation of energy.

To determine the conserved charges, we start with a variational principle: the principle of least action. The evolution of a system between two points corresponds to 
an extremum of the action, $\delta \mathcal{S}=0[\mathbf{9 0}$. This leads to the Euler-Lagrange equations,

$$
\partial_{\mu}\left(\frac{\partial \mathcal{L}}{\partial\left(\partial_{\mu} \phi_{i}\right)}\right)-\frac{\partial \mathcal{L}}{\partial \phi_{i}}=0
$$

which are the equations of motion of the system. Consider a transformation,

$$
\phi \rightarrow \phi+\alpha \delta \phi
$$

where $\alpha$ is some small parameter and $\delta \phi$ is a small variation about $\phi$. For this to be a symmetry, the Lagrangian describing the system must be invariant under this transformation up to a four-divergence,

$$
\mathcal{L} \rightarrow \mathcal{L}+\alpha \partial_{\mu} K^{\mu} \equiv \mathcal{L}+\alpha \delta \mathcal{L}
$$

for some field $K^{\mu}$. If we examine the variation of the Lagrangian itself, we find

$$
\begin{aligned}
\alpha \delta \mathcal{L} & =\frac{\partial \mathcal{L}}{\partial \phi} \alpha \delta \phi+\left(\frac{\partial \mathcal{L}}{\partial\left(\partial_{\mu} \phi\right)}\right) \partial_{\mu}(\alpha \delta \phi) \\
& =\frac{\partial \mathcal{L}}{\partial \phi} \alpha \delta \phi+\partial_{\mu}\left[\frac{\partial \mathcal{L}}{\partial\left(\partial_{\mu} \phi\right)} \alpha \delta \phi\right]-\partial_{\mu}\left(\frac{\partial \mathcal{L}}{\partial\left(\partial_{\mu} \phi\right)}\right) \alpha \delta \phi \\
& =\partial_{\mu}\left[\frac{\partial \mathcal{L}}{\partial\left(\partial_{\mu} \phi\right)} \alpha \delta \phi\right]+\left[\partial_{\mu}\left(\frac{\partial \mathcal{L}}{\partial\left(\partial_{\mu} \phi\right)}\right)-\frac{\partial \mathcal{L}}{\partial \phi}\right] \alpha \delta \phi \\
& =\partial_{\mu}\left[\frac{\partial \mathcal{L}}{\partial\left(\partial_{\mu} \phi\right)} \alpha \delta \phi\right]
\end{aligned}
$$

where, in the second line we have used the product rule in reverse, and the fourth line makes use of Equation (2.7). Next, we set both variations equal to each other,

$$
\begin{aligned}
\partial_{\mu}\left[\frac{\partial \mathcal{L}}{\partial\left(\partial_{\mu} \phi\right)} \alpha \delta \phi\right] & =\alpha \partial_{\mu} K^{\mu} \\
\alpha \partial_{\mu}\left[\frac{\partial \mathcal{L}}{\partial\left(\partial_{\mu} \phi\right)} \delta \phi-K^{\mu}\right] & =0 .
\end{aligned}
$$


Since $\alpha \neq 0$, the four-divergence must be zero. We have arrived at a continuity equation, where the conserved current is given by the quantity in brackets,

$$
\partial_{\mu} J^{\mu} \equiv \partial_{\mu}\left[\frac{\partial \mathcal{L}}{\partial\left(\partial_{\mu} \phi\right)} \delta \phi-K^{\mu}\right]=0 \text {. }
$$

If we expand the conservation equation in space and time,

$$
\partial_{t} J^{0}+\nabla \cdot \vec{J}=0
$$

and integrate both parts,

$$
\int_{\Omega} d^{3} x \frac{d J^{0}}{d t}=-\int_{\Omega} d^{3} x \nabla \cdot \vec{J}=-\int_{\partial \Omega} d \vec{a} \cdot \vec{J} .
$$

If we consider $\Omega$ to be a very large volume (infinity, say), the right-hand side of this expression vanishes. Then

$$
\frac{d}{d t} \int_{\Omega} d^{3} x J^{0}=0 \quad \Longrightarrow \quad \int_{\Omega} d^{3} x J^{0}=Q,
$$

for some constant $Q$. It is this $Q$ which is the conserved charge: the integral is over all space, but is constant in time.

The gauge group of the $\mathrm{SM}$ is $S U(3)_{c} \otimes S U(2)_{L} \otimes U(1)_{Y}$, where

- $S U(3)_{c}$ describes the strong interactions between particles with colour charge;

- $S U(2)_{L}$ describes the weak interactions between particles with isospin charge; and

- $U(1)_{Y}$ describes the hypercharge interactions between particles with weak hypercharge.

The SM Lagrangian is invariant under transformations of these three groups. However, for (certain) particles to acquire mass, the Higgs mechanism breaks the $S U(2)_{L} \otimes$ 
$U(1)_{Y}$ symmetry (as described in Section 4.2), resulting in $U(1)_{\mathrm{EM}}$. This is the group that describes electromagnetism with conserved electric charge. Colour is another charge which is conserved in all interactions. We would like to extend the SM to incorporate a DM candidate, and a convenient place to do so (since we are interested in WIMPs) is in the unbroken electroweak sector.

\subsection{The electroweak sector: Isospin and hypercharge}

In the broken, low-energy phase, the $\mathrm{SM}$ has the gauge group $S U(3)_{c} \otimes U(1)_{\mathrm{EM}}$, where the conserved charges are colour - in $S U(3)_{c}$ - and electric - in $U(1)_{\mathrm{EM}}$. In the unbroken phase, we have the gauge group $S U(3)_{c} \otimes S U(2)_{L} \otimes U(1)_{Y}$. There is again a conserved colour charge, but now electric charge shows that it is a composite charge made up of weak isospin - the conserved charge of $S U(2)_{L}$ - and weak hyperchargethe conserved charge of $U(1)_{Y}$. Hypercharge, like electric charge, corresponds to a one-parameter group, and so is given by a single generator, $Y$. Isospin, on the other hand, is a three-parameter group with generators $T^{a}$. We encounter the (fundamental) two-dimensional representation of $S U(2)$ in the case of spin in elementary quantum mechanics. In that case, the generators are given by the Pauli matrices, $T^{a}=\sigma^{a} / 2$. The electric charge is related to the weak isospin and hypercharge through the GellMann-Nishijima formula [91 $]^{1}$,

$$
Q=T^{3}+\frac{Y}{2}
$$

The values of weak isospin, hypercharge, and electric charge for a single generation of SM particles are given in Table 2.2. Notice that the left-handed quarks, $u_{L}$ and $d_{L}$, differ only in their assignments of $T^{3}$ which is $\pm 1 / 2$. It turns out that they form a

\footnotetext{
${ }^{1}$ There is also another hypercharge normalization such that $Q=T^{3}+Y$. Throughout this thesis, we work in the normalization given in Equation (2.16).
} 


\begin{tabular}{c|c|c|c}
\hline \hline fermion & $T^{3}$ & $Y$ & $Q$ \\
\hline$u_{L}$ & $1 / 2$ & $1 / 3$ & $2 / 3$ \\
$d_{L}$ & $-1 / 2$ & $1 / 3$ & $-1 / 3$ \\
$u_{R}$ & 0 & $4 / 3$ & $2 / 3$ \\
$d_{R}$ & 0 & $-2 / 3$ & $-1 / 3$ \\
$\nu_{L}$ & $1 / 2$ & -1 & 0 \\
$e_{L}$ & $-1 / 2$ & -1 & -1 \\
$e_{R}$ & 0 & -2 & -1 \\
\hline \hline
\end{tabular}

TABLE 2.2. Quantum numbers for a single generation of SM fermions. In this table, $T^{3}$ is the third component of (weak) isospin, $Y$ is the (weak) hypercharge, and $Q=T^{3}+Y / 2$ is the particle's electric charge.

doublet of $S U(2)_{L}$,

$$
Q_{L}=\left(\begin{array}{l}
u_{L} \\
d_{L}
\end{array}\right)
$$

Similarly, for the left-handed leptons,

$$
L_{L}=\left(\begin{array}{c}
\nu_{L} \\
e_{L}
\end{array}\right)
$$

The right-handed quarks and leptons $-u_{R}, d_{R}$, and $e_{R}$ - each form singlets of $S U(2)_{L}$. That is, they do not experience the $S U(2)_{L}$ but, since they carry hypercharge, they do experience $U(1)_{Y}$.

The force carriers have representation assignments as given in Table 2.3. Note that the gluons, $G$, form an octet under $S U(3)_{c}$, the Higgs is a doublet of $S U(2)_{L}$, and the $W$ are a triplet of $S U(2)_{L}$. 


\begin{tabular}{c|c|c|c}
\hline \hline boson & $S U(3)_{c}$ & $S U(2)_{L}$ & $U(1)_{Y}$ \\
\hline$B$ & $\mathbf{1}$ & $\mathbf{1}$ & $\mathbf{0}$ \\
$W^{a}$ & $\mathbf{1}$ & $\mathbf{3}$ & $\mathbf{0}$ \\
$G^{i}$ & $\mathbf{8}$ & $\mathbf{1}$ & $\mathbf{0}$ \\
$\Phi^{a}$ & $\mathbf{1}$ & $\mathbf{2}$ & $\mathbf{1}$ \\
\hline \hline
\end{tabular}

TABLE 2.3. Group representations for the SM gauge bosons. The superscript $a$ corresponds to the $S U(2)_{L}$ index, while $i$ corresponds to the $S U(3)_{c}$ index. The $\Phi$ is the Higgs doublet, $G^{i}$ are the gluons, and (once electroweak symmetry is broken, as described in Section 4.2) the $B$ and $W^{a}$ mix to form the $W^{ \pm}, Z$, and $\gamma$.

\subsection{Extending the electroweak sector}

As we noted in the previous section, the same group used to explain isospin, $S U(2)$, is also that used in quantum mechanics to describe spin. Although the generators of the fundamental representation of $S U(2)_{L}$ can be given as $2 \times 2$ matrices $T^{a}$ $(a=1,2,3)$, this is not the only representation possible. The SM also contains singlets and triplets of $S U(2)_{L}$ (see Table 2.3). In general, we may have the generators as $n \times n$ matrices $T^{a}(a=1,2,3)$, which satisfy the commutation relations $\mathbf{9 2}$

$$
\left[T^{a}, T^{b}\right]=i \epsilon^{a b c} T^{c}, \quad\left[(T)^{2}, T^{a}\right]=0, \quad(T)^{2}=\left(T^{1}\right)^{2}+\left(T^{2}\right)^{2}+\left(T^{3}\right)^{2},
$$

where $\epsilon^{a b c}$ is the completely antisymmetric tensor. The basis vectors of this representation is written as $\left|T ; t^{3}\right\rangle$ where

$$
(T)^{2}\left|T ; t^{3}\right\rangle=T(T+1)\left|T ; t^{3}\right\rangle, \quad T^{3}\left|T ; t^{3}\right\rangle=t^{3}\left|T ; t^{3}\right\rangle
$$

and $t^{3}=-T,-T+1, \ldots, T-1, T$. The collection of basis vectors, $\left|T ; t^{3}\right\rangle$, is collectively known as a multiplet. The size of the multiplet, $n$, is simply the number 
of basis vectors,

$$
n=2 T+1 .
$$

Informally, the particles of a multiplet share all the same properties except for one. In this case, the members of the multiplet carry different values of $T^{3}$.

Using the commutation relations for $T^{ \pm}$, we note that

$$
T^{ \pm} T^{\mp}=(T)^{2}-\left(T^{3}\right)^{2} \pm T^{3}
$$

and so, acting on a state $\left|T ; t^{3}\right\rangle$,

$$
\left(T^{+} T^{-}+T^{-} T^{+}\right)\left|T ; t^{3}\right\rangle=2\left[T(T+1)-\left(t^{3}\right)^{2}\right]\left|T ; t^{3}\right\rangle .
$$

Under the group $S U(2)_{L} \otimes U(1)_{Y}$, all members of a multiplet will have the same value of hypercharge, $Y$, but different values of weak isospin, $T^{3}$. Hence, in the broken $U(1)_{\mathrm{EM}}$, they may be differentiated by their electric charge, using Equation (2.16).

Spin and isospin are independent: it is possible to have scalar (spin-0), fermion (spin-1/2), or vector (spin-1) multiplets. We know, for example, that the $W$ bosons form a 3 under $S U(2)_{L}$, that is, a vector triplet of $S U(2)_{L}$. Supersymmetric gauge theories are the most prevalent extensions of the SM that make use of vector multiplets [93]. Extensions of the SM that involve fermion multiplets of $S U(2)_{L}$ are often used as models of DM, and to explain neutrino masses [94].

Extensions of the SM Higgs sector involving an additional "inert" scalar multiplet, the lightest state of which is stable and hence a possible DM candidate, have been well-studied in the singlet [95, 96, 97, 98, doublet [99], and triplet [100] cases (for recent summaries of the experimental status of these models, see, e.g., Refs. [101, 102, 103]). More recently, multiplets from larger representations of $S U(2)_{L}$ have 
been investigated in the context of dark matter [56, 57, 58, 84], which examined both scalar and fermion multiplets.

\subsection{Large electroweak scalar multiplets}

The large electroweak scalar multiplets are a simple and nearly minimal extension of the SM. They constitute related fields transforming as an $n$-plet of $S U(2)_{L}$ and may contain a dark matter candidate. In general, a large multiplet, $\Sigma$, is a colourless isospin $n$-plet with hypercharge $Y_{\Sigma}$. This means that the large multiplet carries no colour charge, but does carry both electroweak charges. The value of $n$ describes the size of the large multiplet (and is related to the total isospin of the multiplet, $n=2 T+1$ ), while $Y_{\Sigma}$ is the value of the weak hypercharge for all members of the multiplet. The large multiplet $\Sigma$ can be written as

$$
\Sigma=\left(\begin{array}{c}
x_{n} \\
x_{n-1} \\
\vdots \\
x_{2} \\
x_{1}
\end{array}\right)
$$

The fields that make up $\Sigma$ are, in general, complex-valued, meaning $x^{*} \neq x$. Realvalued multiplets are possible, but we do not discuss them here. The multiplets we will examine are scalars, and hence do not carry spin,

$$
S x_{j}=0
$$

for each $j \in[1, n]$. 
Their electric charge is related to the third component of isospin and weak hypercharge of the multiplet member using the Gell-Mann-Nishijima formula, Equation (2.16)

$$
Q x_{j}=\left(T_{j}^{3}+\frac{Y_{\Sigma}}{2}\right) x_{j}
$$

The generators of $S U(2)$ for $n=2,3, \ldots, 8$ are given in Appendix C. Through a judicious choice of $T^{3}$ and $Y_{\Sigma}$, it is possible for one member of the large multiplet, $x_{k}$, for some $k \in[1, n]$, to be electrically neutral. Let $Y_{\Sigma}=-2 T_{k}^{3}$, then

$$
Q x_{k}=\left(T_{k}^{3}+\frac{Y_{\Sigma}}{2}\right) x_{k}=\left(T_{k}^{3}-T_{k}^{3}\right) x_{k}=0
$$

If we further impose that the mass of $x_{k}$ is less than all other multiplet member masses, then we have the beginnings of an ideal DM candidate.

\subsection{Constraints on multiplet size and hypercharge}

The first question we may ask of these large multiplets is just how large is "large"? To answer that, we must consider partial wave unitarity for the annihilation of multiplet members into a pair of gauge bosons. The Lagrangian that describes the interactions between the large multiplets and the gauge bosons is

$$
\mathcal{L}=\left(\mathcal{D}_{\mu} \Sigma\right)^{\dagger}\left(\mathcal{D}^{\mu} \Sigma\right)
$$

where the covariant derivative is given by

$$
\begin{aligned}
\mathcal{D}_{\mu} & =\partial_{\mu}-i g W_{\mu}^{a} T^{a}-i g^{\prime} B_{\mu} \frac{Y}{2} \\
& =\partial_{\mu}-i \frac{g}{\sqrt{2}}\left(W_{\mu}^{+} T^{+}+W_{\mu}^{-} T^{-}\right)-i g W_{\mu}^{3} T^{3}-i g^{\prime} B_{\mu} \frac{Y}{2} .
\end{aligned}
$$




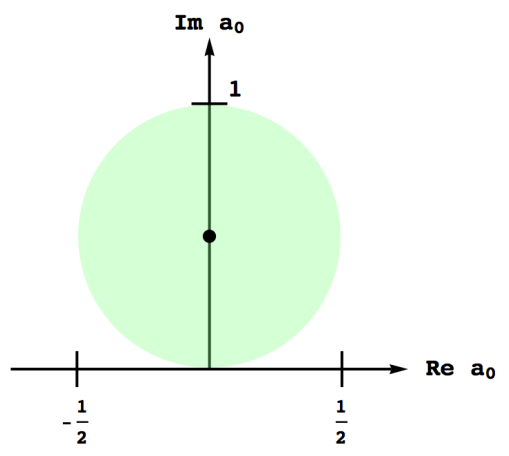

Figure 2.2. Argand diagram showing the unitarity circle. By expanding the matrix element in partial waves, the optical theorem tells us that the real part of the zeroth partial wave amplitude must be less than or equal to one-half.

A general matrix element can be expanded in partial waves,

$$
\mathcal{M}=16 \pi \sum_{j}(2 j+1) a_{j} P_{j}(\cos \theta)
$$

where $j$ is the orbital angular momentum of the final state and $P_{j}(\cos \theta)$ are the Legendre polynomials. From unitarity of the $S$-matrix and the optical theorem, we have 104

$$
\operatorname{Im} a_{j} \geq\left|a_{j}\right|^{2}=\left(\operatorname{Re} a_{j}\right)^{2}+\left(\operatorname{Im} a_{j}\right)^{2} .
$$

Re-arranging, we find

$$
\left(\operatorname{Re} a_{j}\right)^{2} \leq \operatorname{Im} a_{j}\left(1-\operatorname{Im} a_{j}\right)
$$

The right-hand side is a quadratic in $\operatorname{Im} a_{j}$, which has a maximum of $1 / 4$ when $\operatorname{Im} a_{j}=1 / 2$. Thus,

$$
\left|\operatorname{Re} a_{j}\right| \leq \frac{1}{2}
$$

We can express this graphically, as in Figure 2.2.

We will place limits on the zeroth partial wave amplitude, $a_{0}$ [1]. Figure 2.3 shows the possible diagrams involved in annihilating any multiplet member and its 


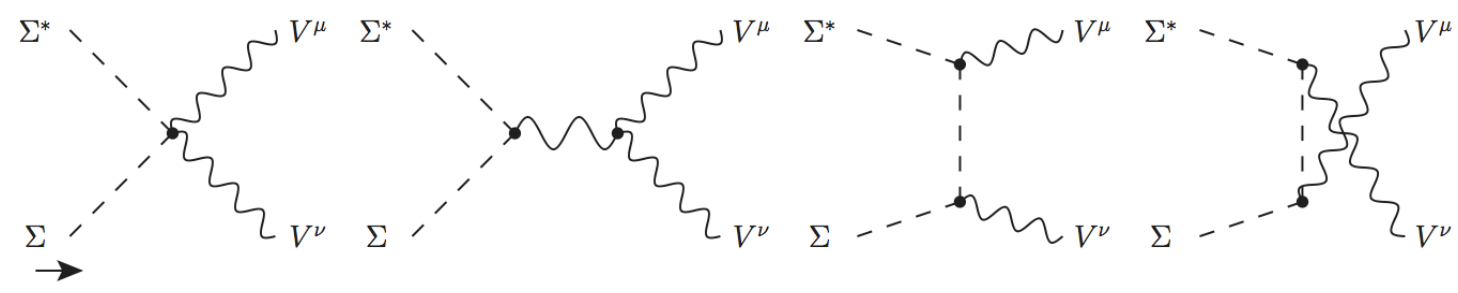

Figure 2.3. Feynman diagrams contributing to the annihilation of two multiplet members into two gauge bosons. In these diagrams, $\Sigma$ represents any member of the multiplet, and $V^{\alpha} \in\left\{W^{ \pm \alpha}, W^{3 \alpha}, B^{\alpha}\right\}$.

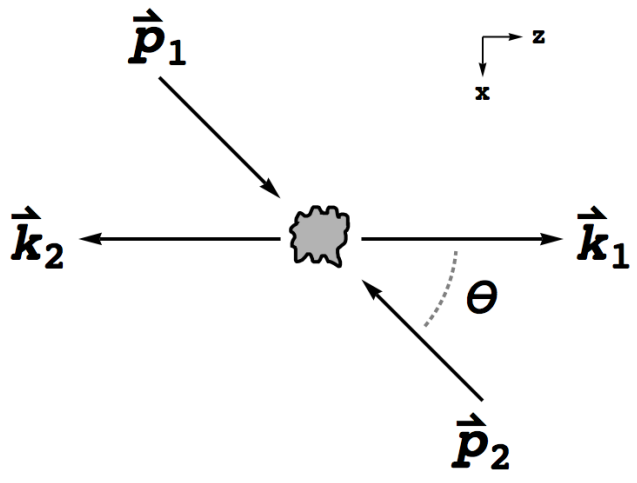

Figure 2.4. Kinematics for the process $\Sigma\left(p_{1}\right) \Sigma^{*}\left(p_{2}\right) \rightarrow V^{\mu}\left(k_{1}\right) V^{\nu}\left(k_{2}\right)$. In this diagram, $\vec{p}_{1} \& \vec{p}_{2}$ are the 3 -momenta of the incoming particles, and $\vec{k}_{1} \& \vec{k}_{2}$ are the 3 -momenta of the outgoing particles.

antiparticle, $\Sigma$ and $\Sigma^{*}$ respectively, into two gauge bosons, $V^{\mu}$ and $V^{\nu}$. We would like to separate constraints arising from each of $S U(2)_{L}$ and $U(1)_{Y}$, and so we work in the unbroken basis of $\left\{W^{ \pm}=\left(W^{1} \pm W^{2}\right) / \sqrt{2}, W^{3}, B\right\}$.

We consider the scattering $\Sigma\left(p_{1}\right) \Sigma^{*}\left(p_{2}\right) \rightarrow V_{1}^{\mu}\left(k_{1}\right) V_{2}^{\nu}\left(k_{1}\right)$ in the $x z$-plane, where the four-momenta are given by

$$
\begin{array}{ll}
p_{1}^{\mu}=\left(E_{p_{1}},\left|\vec{p}_{1}\right| \sin \theta, 0,\left|\vec{p}_{1}\right| \cos \theta\right), & k_{1}^{\mu}=\left(E_{k_{1}}, 0,0,\left|\vec{k}_{1}\right|\right), \\
p_{2}^{\mu}=\left(E_{p_{2}},-\left|\vec{p}_{2}\right| \sin \theta, 0,-\left|\vec{p}_{2}\right| \cos \theta\right), & k_{2}^{\mu}=\left(E_{k_{2}}, 0,0,-\left|\vec{k}_{2}\right|\right),
\end{array}
$$


and the polarization vectors of the gauge boson are given by

$$
\begin{aligned}
\epsilon_{\mathrm{in}}^{\mu}\left(k_{1}\right) & =(0,1,0,0), \quad \epsilon_{\mathrm{in}}^{\mu}\left(k_{2}\right)=(0,1,0,0), \\
\epsilon_{\text {out }}^{\mu}\left(k_{1}\right) & =(0,0,1,0), \quad \epsilon_{\text {out }}^{\mu}\left(k_{2}\right)=(0,0,-1,0),
\end{aligned}
$$

where the subscripts "in" and "out" refer to polarizations in and out of the plane.

We start with the annihilation to hypercharge bosons, $\Sigma\left(p_{1}\right) \Sigma^{*}\left(p_{2}\right) \rightarrow B^{\mu}\left(k_{1}\right) B^{\nu}\left(k_{2}\right)$. The matrix elements for the annihilation processes Figure 2.3 there is no $s$-channel process in this case) are given by

$$
\begin{aligned}
\mathcal{M}\left(\Sigma^{*} \Sigma \rightarrow B B\right)_{4 \mathrm{pt}} & =g^{\prime 2} \frac{Y_{\Sigma}^{2}}{2} \epsilon^{\mu}\left(k_{1}\right) \epsilon_{\mu}\left(k_{2}\right) \\
\mathcal{M}\left(\Sigma^{*} \Sigma \rightarrow B B\right)_{t} & =-g^{\prime 2} \frac{Y_{\Sigma}^{2}}{4}\left(p_{1}+q\right)^{\mu} \epsilon_{\mu}\left(k_{1}\right)\left(q-p_{2}\right)^{\nu} \epsilon_{\nu}\left(k_{2}\right) \frac{1}{q^{2}-m_{i}^{2}} \\
\mathcal{M}\left(\Sigma^{*} \Sigma \rightarrow B B\right)_{u} & =-g^{\prime 2} \frac{Y_{\Sigma}^{2}}{4}\left(q^{\prime}-p_{2}\right)^{\mu} \epsilon_{\mu}\left(k_{1}\right)\left(p_{1}+q^{\prime}\right)^{\nu} \epsilon_{\nu}\left(k_{2}\right) \frac{1}{q^{\prime 2}-m_{i}^{2}}
\end{aligned}
$$

where $q=p_{1}-k_{1}=k_{2}-p_{2}$ and $q^{\prime}=p_{1}-k_{2}=k_{1}-p_{2}$ are the $t$ - and $u$-channel propagator momenta respectively. Adding these together and making use of the transverse polarizations given in Equation (2.35), we get the total scattering matrix element. For both bosons polarized out of the plane, we get

$$
\mathcal{M}_{B B}^{\perp}=\mathcal{M}_{4 \mathrm{pt}}^{\perp}+\mathcal{M}_{t}^{\perp}+\mathcal{M}_{u}^{\perp}=g^{\prime 2} \frac{Y_{\Sigma}^{2}}{2}+0+0=g^{\prime 2} \frac{Y_{\Sigma}^{2}}{2}
$$

while for both bosons polarized in the plane, we get

$$
\begin{gathered}
\mathcal{M}\left(\Sigma^{*} \Sigma \rightarrow B B\right)_{4 \mathrm{pt}}^{\|}=-g^{\prime 2} \frac{Y_{\Sigma}^{2}}{2} \\
\mathcal{M}\left(\Sigma^{*} \Sigma \rightarrow B B\right)_{t}^{\|}=-g^{\prime 2} Y_{\Sigma}^{2} \frac{\left|\vec{p}_{1}\right|\left|\vec{p}_{2}\right| \sin ^{2} \theta}{q^{2}-m_{i}^{2}} \\
\mathcal{M}\left(\Sigma^{*} \Sigma \rightarrow B B\right)_{u}^{\|}=-g^{\prime 2} Y_{\Sigma}^{2} \frac{\left|\vec{p}_{1}\right|\left|\vec{p}_{2}\right| \sin ^{2} \theta}{q^{\prime 2}-m_{i}^{2}}
\end{gathered}
$$


Working in the centre-of-momentum frame, we have the following

$$
\begin{aligned}
\left|\vec{p}_{1}\right|=\left|\vec{p}_{2}\right| & =\frac{\sqrt{s}}{2}, \\
q^{2}=t & =-\frac{s(1-\cos \theta)}{2}, \\
q^{\prime 2}=u & =-\frac{s(1+\cos \theta)}{2} .
\end{aligned}
$$

In the high-energy limit, $q^{2}, q^{2} \gg m_{i}^{2}$, we can simplify the matrix elements of Equation (2.38)

$$
\begin{aligned}
\mathcal{M}\left(\Sigma^{*} \Sigma \rightarrow B B\right)_{t}^{\|} & =-g^{\prime 2} Y_{\Sigma}^{2} \frac{\left|\vec{p}_{1}\right|\left|\vec{p}_{2}\right| \sin ^{2} \theta}{q^{2}-m_{i}^{2}} \\
& \approx-g^{\prime 2} Y_{\Sigma}^{2} \frac{(\sqrt{s} / 2)(\sqrt{s} / 2)(1+\cos \theta)(1-\cos \theta)}{-\frac{s(1-\cos \theta)}{2}} \\
& =g^{\prime 2} \frac{Y_{\Sigma}^{2}}{2}(1+\cos \theta)
\end{aligned}
$$

Similarly,

$$
\mathcal{M}\left(\Sigma^{*} \Sigma \rightarrow B B\right)_{u}^{\|} \approx g^{\prime 2} \frac{Y_{\Sigma}^{2}}{2}(1-\cos \theta)
$$

Adding together, we find in the high-energy limit,

$$
\mathcal{M}_{B B}^{\|}=-g^{\prime 2} \frac{Y_{\Sigma}^{2}}{2}+g^{\prime 2} \frac{Y_{\Sigma}^{2}}{2}(1+\cos \theta)+g^{\prime 2} \frac{Y_{\Sigma}^{2}}{2}(1-\cos \theta)=g^{\prime 2} \frac{Y_{\Sigma}^{2}}{2}
$$

Regardless of whether the bosons are both polarized out of or in the plane, they give the same scattering matrix element. Notice that if the polarizations of the bosons are perpendicular, the matrix elements all vanish.

The scattering matrix elements to $W^{3} B$ and $W^{3} W^{3}$ can be computed in the same manner, or by noting that the only difference is the couplings and making the 
substitutions

$$
\begin{array}{ll}
\left(g^{\prime} \frac{Y_{\Sigma}}{2}\right)^{2} \rightarrow\left(g^{\prime} \frac{Y_{\Sigma}}{2}\right)\left(g T_{i}^{3}\right) & \text { for } W^{3} B, \\
\left(g^{\prime} \frac{Y_{\Sigma}}{2}\right)^{2} \rightarrow\left(g T_{i}^{3}\right)^{2} & \text { for } W^{3} W^{3},
\end{array}
$$

where $T_{i}^{3}$ is the third component of isospin of the $i$ th member of $\Sigma$. These substitutions give the total matrix element for each annihilation channel as

$$
\begin{gathered}
\mathcal{M}_{W^{3} B}=g g^{\prime} Y_{\Sigma} T_{i}^{3} \\
\mathcal{M}_{W^{3} W^{3}}=2 g^{2}\left(T_{i}^{3}\right)^{2} .
\end{gathered}
$$

For the annihilation to charged boson pairs, an additional $s$-channel diagram contributes, giving the matrix elements as

$$
\begin{aligned}
& \mathcal{M}_{4 \mathrm{pt}}\left(\Sigma^{*} \Sigma \rightarrow W^{+} W^{-}\right)=\frac{g^{2}}{2}\left[T^{+} T^{-}+T^{-} T^{+}\right] \epsilon^{\mu}\left(k_{1}\right) \epsilon_{\mu}\left(k_{2}\right), \\
& \mathcal{M}_{t}\left(\Sigma^{*} \Sigma \rightarrow W^{+} W^{-}\right)=\frac{g^{2}}{2} T^{+} T^{-} \frac{\left(q+p_{2}\right)^{\mu}\left(p_{1}-q\right)^{\nu} \epsilon_{\mu}\left(k_{1}\right) \epsilon_{\nu}\left(k_{2}\right)}{q^{2}-m_{i-1}^{2}} \\
& \mathcal{M}_{u}\left(\Sigma^{*} \Sigma \rightarrow W^{+} W^{-}\right)=\frac{g^{2}}{2} T^{-} T^{+} \frac{\left(q^{\prime}-p_{1}\right)^{\mu}\left(p_{2}+q^{\prime}\right)^{\nu} \epsilon_{\mu}\left(k_{1}\right) \epsilon_{\nu}\left(k_{2}\right)}{q^{\prime 2}-m_{i-1}^{2}} \\
& \mathcal{M}_{s}\left(\Sigma^{*} \Sigma \rightarrow W^{+} W^{-}\right)=-g^{2} T^{3} \frac{1}{k^{2}}\left[g^{\mu \nu}\left(k_{2}-k_{1}\right)^{\sigma}+2 g^{\mu \sigma} k_{1}^{\nu}-2 g^{\nu \sigma} k_{2}^{\mu}\right] \\
& \times \epsilon_{\mu}\left(k_{1}\right) \epsilon_{\nu}\left(k_{2}\right)\left(p_{1}-p_{2}\right)_{\sigma} .
\end{aligned}
$$

Using the polarizations of Equation (2.35), with both bosons polarized out of the plane, the only contributions are from $\mathcal{M}_{4 \mathrm{pt}}$ and $\mathcal{M}_{s}$, where $k^{2}=\left(p_{1}+p_{2}\right)^{2}=s$ and $\left(p_{1}-p_{2}\right) \cdot\left(k_{2}-k_{1}\right)=s \cos \theta$

$$
\begin{aligned}
\mathcal{M}_{4 \mathrm{pt}}^{\perp} & =g^{2}\left[T(T+1)-\left(T^{3}\right)^{2}\right], \\
\mathcal{M}_{s}^{\perp} & =-g^{2} T^{3} \cos \theta .
\end{aligned}
$$


However, note that $\mathcal{M}_{s}^{\perp} \propto \cos \theta \sim P_{1}(\cos \theta)$, and so contributes to $a_{1}$ but not $a_{0}$. Thus,

$$
\mathcal{M}_{W^{ \pm} W^{\mp}}^{0, \perp}=g^{2}\left[T(T+1)-\left(T^{3}\right)^{2}\right]
$$

Now, if we set one polarization in the plane and the other out of the plane, all diagrams give zero. Finally, if we set both polarizations in the plane, we get

$$
\begin{aligned}
\mathcal{M}_{4 \mathrm{pt}}^{\|} & =-g^{2}\left[T(T+1)-\left(T^{3}\right)^{2}\right], \\
\mathcal{M}_{t}^{\|} & =g^{2} T^{+} T^{-}(1+\cos \theta) \\
\mathcal{M}_{u}^{\|} & =g^{2} T^{-} T^{+}(1-\cos \theta), \\
\mathcal{M}_{s}^{\|} & =g^{2} T^{3} \cos \theta .
\end{aligned}
$$

Adding these together, and dropping the terms proportional to $\cos \theta$ (which contribute to $a_{1}$ but not $\left.a_{0}\right)$,

$$
\mathcal{M}_{W^{ \pm} W^{\mp}}^{0, \|}=g^{2}\left[T(T+1)-\left(T^{3}\right)^{2}\right]
$$

which is equivalent to Equation (2.47)

Summarizing, the total matrix elements for the annihilation of two large multiplet members into two SM gauge bosons are given by Equations (2.42), (2.44) and (2.47), Collecting these together, we have

$$
\begin{aligned}
\mathcal{M}^{0}\left(\Sigma^{*} \Sigma \rightarrow B B\right) & =g^{\prime 2} \frac{Y_{\Sigma}^{2}}{2}, \\
\mathcal{M}^{0}\left(\Sigma^{*} \Sigma \rightarrow W^{3} B\right) & =g g^{\prime} Y_{\Sigma} T_{i}^{3}, \\
\mathcal{M}^{0}\left(\Sigma^{*} \Sigma \rightarrow W^{3} W^{3}\right) & =2 g^{2}\left(T_{i}^{3}\right)^{2}, \\
\mathcal{M}^{0}\left(\Sigma^{*} \Sigma \rightarrow W^{ \pm} W^{\mp}\right) & =g^{2}\left[T(T+1)-\left(T_{i}^{3}\right)^{2}\right],
\end{aligned}
$$


where $T$ is the total isospin of the multiplet, $Y_{\Sigma}$ is the hypercharge of the multiplet, and $T_{i}^{3}$ is the third component of isospin of the $i$ th multiplet member.

Recall the unitarity bound condition,

$$
\left|\operatorname{Re} a_{0}\right| \leq \frac{1}{2}
$$

If there exist amplitudes that couple the same initial or final state to multiple final or initial states, respectively, the strongest bound comes from applying Equation (2.51) to the largest of those amplitudes. This is done by diagonalizing a matrix formed by the coupled channels and applying Equation (2.51) to the largest eigenvalue. In this way, we perform a coupled-channel analysis.

The zeroth partial wave amplitude can be obtained from Equation (2.30),

$$
a_{0}=\frac{1}{16 \pi} \mathcal{M}^{0}\left(\Sigma^{*} \Sigma \rightarrow V_{1} V_{2}\right)
$$

where $V_{i} \in\left\{B, W^{3}, W^{+}, W^{-}\right\}$. From Equation (2.50), we find

$$
\begin{aligned}
a_{0}\left(\Sigma_{i} \Sigma_{i}^{*} \rightarrow B B / \sqrt{2}\right) & =\frac{g^{2}}{16 \pi} \frac{s_{W}^{2}}{c_{W}^{2}} \frac{Y_{\Sigma}^{2}}{2 \sqrt{2}}, \\
a_{0}\left(\Sigma^{*} \Sigma \rightarrow W^{3} B\right) & =\frac{g^{2}}{16 \pi} \frac{s_{W}}{c_{W}} Y_{\Sigma} T_{i}^{3}, \\
a_{0}\left(\Sigma^{*} \Sigma \rightarrow W^{3} W^{3} / \sqrt{2}\right) & =\frac{g^{2}}{16 \pi} \sqrt{2}\left(T_{i}^{3}\right)^{2}, \\
a_{0}\left(\Sigma^{*} \Sigma \rightarrow W^{ \pm} W^{\mp}\right) & =\frac{g^{2}}{16 \pi}\left[T(T+1)-\left(T_{i}^{3}\right)^{2}\right],
\end{aligned}
$$

where $s_{W}$ is the sine of the weak mixing angle, $g^{\prime} / g=s_{W} / c_{W}$, and we have included a factor of $1 / \sqrt{2}$ to account for initial or final states involving identical particles.

For purely $U(1)_{Y}$ interactions, $\Sigma^{*} \Sigma \rightarrow B B$, we note that the zeroth partial wave amplitude is the same for all members of the multiplet. The coupled-channel matrix 
is then given by

$$
\mathbf{a}_{0}^{U(1)_{Y}}=\sqrt{2} \frac{g^{2}}{16 \pi} \frac{s_{W}^{2}}{c_{W}^{2}} \frac{Y_{\Sigma}^{2}}{2 \sqrt{2}}{ }^{B B / \sqrt{2}}\left(\begin{array}{ccccc}
B B / \sqrt{2} & \Sigma_{1} \Sigma_{1}^{*} & \cdots & \Sigma_{n} \Sigma_{n}^{*} \\
0 & 1 & \cdots & 1 \\
& \vdots \\
\Sigma_{n} \Sigma_{n}^{*} & 0 & \cdots & 0 \\
\vdots & \vdots & & \vdots \\
1 & 0 & \cdots & 0
\end{array}\right),
$$

where the additional factor of $\sqrt{2}$ in front comes from the two possible polarization states of the $B$ bosons. Diagonalizing the matrix of integers results in two nonzero eigenvalues, $\pm \sqrt{n}$, and $n-1$ zero eigenvalues. The eigenvectors corresponding to the nonzero eigenvalues are

$$
\frac{1}{\sqrt{2}}\left(\frac{B B}{\sqrt{2}} \pm\left[\Sigma^{*} \Sigma\right]_{0}\right)
$$

where the properly normalized symmetric combination of all $n$ states with zero total isospin is given by

$$
\left[\Sigma^{*} \Sigma\right]_{0} \equiv \frac{1}{\sqrt{n}} \sum_{i} \Sigma_{i}^{*} \Sigma_{i} .
$$

The maximum eigenvalue, $a_{0}^{\mathrm{MAX}, U(1)_{Y}}$, is then given by

$$
a_{0}^{\mathrm{MAX}, U(1)_{Y}}=\frac{g^{2}}{16 \pi} \frac{s_{W}^{2}}{c_{W}^{2}} \frac{Y_{\Sigma}^{2}}{2} \sqrt{n} .
$$

Imposing Equation (2.51), we obtain a constraint on the multiplet's hypercharge, $Y_{\Sigma}$,

$$
\left|Y_{\Sigma}\right| \leq \frac{4 c_{W}}{g s_{W}}\left(\frac{\pi^{2}}{n}\right)^{\frac{1}{4}} \approx \frac{19.84}{n^{\frac{1}{4}}}
$$


For the mixed interaction, $\Sigma^{*} \Sigma \rightarrow W^{3} B$, we obtain a limit on the multiplet's hypercharge of

$$
\left|Y_{\Sigma}\right| \lesssim \frac{1144.12}{\sqrt{n\left(n^{2}-1\right)}}
$$

This limit is much less restrictive than that found in Equation (2.58), So long as $n \lesssim 25$, the purely $U(1)_{Y}$ interaction will provide the most stringent constraint on the hypercharge.

In the basis $\left\{W^{+} W^{-}, W^{3} W^{3} / \sqrt{2}, \Sigma_{1}^{*} \Sigma_{1}, \ldots, \Sigma_{n}^{*} \Sigma_{n}\right\}$, the purely $S U(2)_{L}$ interactions will form a coupled-channel matrix

$$
\mathbf{a}_{0}^{S U(2)_{L}}=\sqrt{2} \frac{g^{2}}{16 \pi}{ }^{W^{+} W^{-}} \begin{gathered}
\Sigma_{1}^{*} \Sigma_{1} \\
\vdots \\
\Sigma_{n}^{*} \Sigma_{n}
\end{gathered}\left(\begin{array}{cccccc}
W^{+} W^{-} & W^{3} W^{3} / \sqrt{2} & \Sigma_{1}^{*} \Sigma_{1} & \cdots & \Sigma_{n}^{*} \Sigma_{n} \\
0 & 0 & A_{1} & \cdots & A_{n} \\
0 & 0 & B_{1} & \cdots & B_{n} \\
A_{1} & B_{1} & 0 & \cdots & 0 \\
\vdots & \vdots & \vdots & & \vdots \\
A_{n} & B_{n} & 0 & \cdots & 0
\end{array}\right)
$$

where, unlike the $U(1)_{Y}$ case, the scattering amplitude is dependent on which member of the multiplet is scattering. The matrix entries are given by

$$
A_{i}=T(T+1)-\left(T_{i}^{3}\right)^{2}, \quad B_{i}=\sqrt{2}\left(T_{i}^{3}\right)^{2},
$$

with, as usual, $T$ the total isospin of the multiplet and $T_{i}^{3}$ being the third component of isospin for the $i$ th multiplet member. This matrix has $n-2$ zero eigenvalues and two pairs of nonzero eigenvalues. The largest is given by $\pm a_{0}^{\mathrm{MAX}, S U(2)_{L}}$, such that

$$
a_{0}^{\mathrm{MAX}, S U(2)_{L}}=\frac{g^{2}}{16 \pi} 2 T(T+1) \sqrt{\frac{n}{3}}=\frac{g^{2}}{16 \pi} \frac{\left(n^{2}-1\right) \sqrt{n}}{2 \sqrt{3}} .
$$


The eigenvectors corresponding to $\pm a_{0}^{\mathrm{MAX}, S U(2)_{L}}$ are then

$$
\frac{1}{\sqrt{2}}\left([W W]_{0} \pm\left[\Sigma^{*} \Sigma\right]_{0}\right)
$$

where

$$
[W W]_{0} \equiv \frac{1}{\sqrt{6}}\left[W^{1} W^{1}+W^{2} W^{2}+W^{3} W^{3}\right]
$$

and $\left[\Sigma^{*} \Sigma\right]_{0}$ is the total isospin-zero combination given in Equation (2.56),

Applying Equation (2.51) to Equation (2.62), we find a limit on the size of the multiplet, $n$, given by

$$
\left(n^{2}-1\right) \sqrt{n} \leq \frac{16 \pi}{g^{2}} \sqrt{3}
$$

where $g=\sqrt{4 \pi \alpha_{\mathrm{EM}}} / s_{W} \approx 0.651$. This can be solved numerically, recalling that $n$ is an integer, yielding

$$
n \leq 8
$$

We have now determined an upper-bound on the hypercharge and size of large electroweak scalar multiplets.

\subsection{Chapter summary}

In this chapter, we briefly discussed the mathematical structure of the Standard Model of particle physics and, in particular, representations of the electroweak sector. We then extended the SM by the addition of a single large electroweak scalar mul-

tiplet. Using the scattering of multiplet members to pairs of gauge bosons, we were able to set limits on the hypercharge (Equation (2.58) $)$ and the size (Equation (2.66) 


\begin{tabular}{l|cccccccc}
\hline \hline$n$ & 1 & 2 & 3 & 4 & 5 & 6 & 7 & 8 \\
\hline$T$ & 0 & $1 / 2$ & 1 & $3 / 2$ & 2 & $5 / 2$ & 2 & $7 / 2$ \\
\hline$\left|Y_{\Sigma}^{\mathrm{MAX}}\right|$ & 19.8 & 16.7 & 15.1 & 14.0 & 13.0 & 12.1 & 10.8 & 8.3 \\
\hline
\end{tabular}

TABLE 2.4. Maximum hypercharge, weak isospin, and size of large multiplets. The hypercharge is bounded by Equation (2.58), The isospin can be determined using $n=2 T+1$. The size is bounded by Equation (2.66)

of such multiplets. Algebraically, we have

$$
\begin{aligned}
\left|Y_{\Sigma}\right| & \leq \frac{4 c_{W}}{g s_{W}}\left(\frac{\pi^{2}}{n}\right)^{\frac{1}{4}} \\
n & \leq 8 \\
T & \leq \frac{7}{2}
\end{aligned}
$$

Numerically, we find the limits as given in Table 2.4. These results were originally published in Ref. [1]. 


\section{CHAPTER 3}

\section{$Z_{2}$-symmetric models}

In Chapter 2, we described what a large multiplet is, how large it can be (or, equivalently, how large its total isospin can be), and the maximum hypercharge carried by the multiplet. We stated that the multiplet contains a dark matter candidate if the isospin and hypercharge are chosen in a particular way. The Lagrangian in Section 2.5 describes only the interactions between the multiplet members and the gauge bosons of the SM. We would like to include a mass term as well as interactions with the Higgs boson. However, we also want to ensure a DM candidate in our multiplets. This means that we cannot allow the DM candidate to decay. To do this, we must impose a symmetry that forbids such decays. The two simplest symmetries to choose from are $Z_{2}$ and $U(1)$. Models whose Lagrangian preserves a $U(1)$ symmetry are experimentally ruled out, as we showed in Ref. [5], and we have summarized in Appendix E.

In this chapter, we will detail the models whose Lagrangian preserves a $Z_{2}$ symmetry. To obtain these models, we set the hypercharge of the multiplet as $Y_{\Sigma}=1$ and introduce a scalar potential that includes a mass term for the multiplet and interactions with the Higgs boson which will induce mass differences between the states of $\Sigma$. We will begin with a description of the models, their mixing, and mass spectrum. We will then present an alternate parameterization of the models which is more convenient for the collider studies of Chapter 5. We will then describe how the heavier members of the multiplet will decay over the parameter space. 


\subsection{Model description}

The class of models in which we are interested are those large multiplet models whose Lagrangian preserves a $Z_{2}$ symmetry. Simply put, this means that the Lagrangian is invariant under a transformation of the fields, $\varphi \rightarrow-\varphi$. In this case, the large multiplet is denoted $\Sigma$, and is odd under the $Z_{2}$ transformation, while the Standard Model fields are all even:

$$
\Sigma \rightarrow-\Sigma, \quad \Phi \rightarrow \Phi, \quad Q_{L} \rightarrow Q_{L}, \quad W_{\mu}^{ \pm} \rightarrow W_{\mu}^{ \pm}, \quad \text { and so on }
$$

The Lagrangian for this class of models is

$$
\mathcal{L} \supset\left(\mathcal{D}_{\mu} \Sigma\right)^{\dagger}\left(\mathcal{D}^{\mu} \Sigma\right)-V(\Phi, \Sigma)
$$

where $\Phi$ is the SM Higgs doublet, $\Sigma$ is the large multiplet, and $V$ is the scalar potential. The most general scalar potential is given by

$$
\begin{aligned}
V(\Phi, \Sigma) & =\mu^{2} \Phi^{\dagger} \Phi+\lambda_{1}\left(\Phi^{\dagger} \Phi\right)^{2} \\
& +M^{2} \Sigma^{\dagger} \Sigma+\lambda_{2} \Phi^{\dagger} \Phi \Sigma^{\dagger} \Sigma+\lambda_{3} \Phi^{\dagger} T_{\Phi}^{a} \Phi \Sigma^{\dagger} T_{\Sigma}^{a} \Sigma \\
& +\left(\lambda_{4} \widetilde{\Phi}^{\dagger} T_{\Phi}^{a} \Phi \Sigma^{\dagger} T_{\Sigma}^{a} \widetilde{\Sigma}+\text { h.c. }\right)+\left(\lambda_{5}[\Sigma \Sigma]_{3}^{\dagger}[\Phi \Sigma]_{3}+\text { h.c. }\right) \\
& +\lambda_{6}[\Sigma \Sigma]_{1}^{\dagger}[\Sigma \Sigma]_{1}+\lambda_{7}[\Sigma \Sigma]_{3}^{\dagger}[\Sigma \Sigma]_{3} \\
& +\lambda_{8}[\Sigma \Sigma]_{5}^{\dagger}[\Sigma \Sigma]_{5}+\delta_{n 8} \lambda_{9}[\Sigma \Sigma]_{7}^{\dagger}[\Sigma \Sigma]_{7}
\end{aligned}
$$

where $n=6$ or 8 is the size of the multiplet, $T_{\Phi}^{a}$ and $T_{\Sigma}^{a}$ are the $2 \times 2$ and $n \times n S U(2)$

generators, respectively and $\widetilde{\Phi}=i \sigma^{2} \Phi^{*}$ and $\widetilde{\Sigma}=C \Sigma^{*}$ are the conjugate multiplets. The conjugation matrix, $C$, is a skew-symmetric matrix with entries along the antidiagonal alternating between 1 and -1 . The exact form is given in Appendix Calong with the generators $T^{a}$ for $n=2, \ldots, 8$. 
The terms in brackets, $[\Sigma \Sigma]_{T}$, are the total isospin $T$ combinations of pairs of multiplets. They are given in Appendix B. The Kronecker delta, $\delta_{n 8}$ reinforces that the $\lambda_{9}$ term appears only for multiplets of size eight - the maximum possible isospin combination of two $\Sigma$ for $n=6$ is $T+T=5 / 2+5 / 2=5$. For the remainder of this chapter, we will ignore the terms quartic in $\Sigma$, since these terms will not affect the present analysis. We will return to these terms in Sections 4.2 and 4.4 .

We wish for our potential to preserve a $Z_{2}$ symmetry, so we must further impose that $\lambda_{5}=0$ due to the presence of three $\Sigma$ fields. The (slightly reduced) potential which we use for our present analysis takes the form

$$
\begin{aligned}
V(\Phi, \Sigma) & =\mu^{2} \Phi^{\dagger} \Phi+M^{2} \Sigma^{\dagger} \Sigma+\lambda_{1}\left(\Phi^{\dagger} \Phi\right)^{2}+\lambda_{2} \Phi^{\dagger} \Phi \Sigma^{\dagger} \Sigma \\
& +\lambda_{3} \Phi^{\dagger} T_{\Phi}^{a} \Phi \Sigma^{\dagger} T_{\Sigma}^{a} \Sigma+\left(\lambda_{4} \widetilde{\Phi}^{\dagger} T_{\Phi}^{a} \Phi \Sigma^{\dagger} T_{\Sigma}^{a} \widetilde{\Sigma}+\text { h.c. }\right)
\end{aligned}
$$

Why do we consider models containing a multiplet with only six or eight members? From Chapter 2, we know that $n \leq 8$. The $\lambda_{4}$ term of Equation (3.4) couples two $\Phi$ to two $\Sigma^{*}$ (and two $\Phi^{*}$ to two $\Sigma$ ), each pair in an isospin triplet configuration with total hypercharge \pm 2 . If we consider a multiplet with odd $n$, for example $n=5$, the the $\lambda_{4}$ term is identically zero:

$$
\begin{aligned}
& \Sigma^{\dagger} T_{\Sigma}^{3}\left(C \Sigma^{*}\right) \\
& =\left(\zeta^{1 *}, \zeta^{2 *}, \zeta^{3 *}, \zeta^{4 *}, \zeta^{5 *}\right)\left(\begin{array}{ccccc}
2 & 0 & 0 & 0 & 0 \\
0 & 1 & 0 & 0 & 0 \\
0 & 0 & 0 & 0 & 0 \\
0 & 0 & 0 & -1 & 0 \\
0 & 0 & 0 & 0 & -2
\end{array}\right)\left(\begin{array}{ccccc}
0 & 0 & 0 & 0 & -1 \\
0 & 0 & 0 & 1 & 0 \\
0 & 0 & -1 & 0 & 0 \\
0 & 1 & 0 & 0 & 0 \\
-1 & 0 & 0 & 0 & 0
\end{array}\right)\left(\begin{array}{l}
\zeta^{1 *} \\
\zeta^{2 *} \\
\zeta^{3 *} \\
\zeta^{4 *} \\
\zeta^{5 *}
\end{array}\right)
\end{aligned}
$$




$$
\begin{aligned}
& =\left(2 \zeta^{1 *}, \zeta^{2 *}, 0,-\zeta^{4 *},-2 \zeta^{5 *}\right)\left(\begin{array}{c}
-\zeta^{5 *} \\
\zeta^{4 *} \\
-\zeta^{3 *} \\
\zeta^{2 *} \\
-\zeta^{1 *}
\end{array}\right) \\
& =-2 \zeta^{1 *} \zeta^{5 *}+\zeta^{2 *} \zeta^{4 *}-\zeta^{4 *} \zeta^{2 *}+2 \zeta^{5 *} \zeta^{1 *} \\
& =0,
\end{aligned}
$$

and similarly for $\Sigma^{\dagger} T_{\Sigma}^{1}\left(C \Sigma^{*}\right)$ and $\Sigma^{\dagger} T_{\Sigma}^{2}\left(C \Sigma^{*}\right)$. The same holds for $n=7$. As we will see, the $\lambda_{4}$ term is required to break the would-be $U(1)$ symmetry to a $Z_{2}$. This means that the models of interest have $n=6$ or 8 .

In the charge basis, we write the multiplets as

$$
\begin{aligned}
& \Sigma_{(n=6)}=\left(\zeta^{+3}, \zeta^{+2}, \zeta^{+1}, \zeta^{0}, \zeta^{-1}, \zeta^{-2}\right)^{T} \\
& \Sigma_{(n=8)}=\left(\zeta^{+4}, \zeta^{+3}, \zeta^{+2}, \zeta^{+1}, \zeta^{0}, \zeta^{-1}, \zeta^{-2}, \zeta^{-3}\right)^{T}
\end{aligned}
$$

Note that the conjugate state of $\zeta^{Q}$ is written $\zeta^{Q *}$, and that this is not the same as $\zeta^{-Q}$.

Further restricting the size of the multiplet is not the only consequence of the $\lambda_{4}$ term. It splits the neutral component, $\zeta^{0}$, into two real particles: a scalar, $\zeta^{0, r}=$ $\sqrt{2} \operatorname{Re} \zeta^{0}$, and a pseudoscalar, $\zeta^{0, i}=\sqrt{2} \operatorname{Im} \zeta^{0}$. It will also induce mixing between the charged states $\zeta^{+Q}$ and $\zeta^{-Q *}$. The two combinations in the $\lambda_{4}$ terms can be expanded 
using

$$
\begin{aligned}
& \Sigma^{\dagger} T^{-} \widetilde{\Sigma}=\frac{n}{2}(-1)^{\frac{n}{2}+1} \zeta^{0 *} \zeta^{0 *}+\sum_{Q=1}^{n / 2} \sqrt{n^{2}-4 Q^{2}}(-1)^{\frac{n}{2}+Q+1} \zeta^{+Q *} \zeta^{-Q *}, \\
& \widetilde{\Sigma}^{\dagger} T^{+} \Sigma=\frac{n}{2}(-1)^{\frac{n}{2}+1} \zeta^{0} \zeta^{0}+\sum_{Q=1}^{n / 2} \sqrt{n^{2}-4 Q^{2}}(-1)^{\frac{n}{2}+Q+1} \zeta^{+Q} \zeta^{-Q},
\end{aligned}
$$

where $T^{ \pm}=T^{1} \pm i T^{2}$ can be calculated from the generators given in Appendix C. The masses of the neutral states are then

$$
\begin{aligned}
& m_{\zeta^{0, r}}^{2}=M^{2}+\frac{1}{2} v^{2}\left[\lambda_{2}+\frac{\lambda_{3}}{4}+\frac{n}{2}(-1)^{\frac{n}{2}+1} \lambda_{4}\right] \\
& m_{\zeta^{0, i}}^{2}=M^{2}+\frac{1}{2} v^{2}\left[\lambda_{2}+\frac{\lambda_{3}}{4}+\frac{n}{2}(-1)^{\frac{n}{2}} \lambda_{4}\right]=m_{\zeta^{0, r}}^{2}+\frac{n}{2}(-1)^{\frac{n}{2}} v^{2} \lambda_{4} .
\end{aligned}
$$

The mass eigenstates of charge $Q, H_{k}^{Q}(k=1,2)$, are defined for $Q>0\left(H_{k}^{Q *}=\right.$ $\left.H_{k}^{-Q}\right)$ in terms of a mixing angle, $\alpha_{Q}$, such that

$$
\begin{gathered}
\zeta^{Q}=\cos \alpha_{Q} H_{1}^{Q}-\sin \alpha_{Q} H_{2}^{Q}, \\
\zeta^{-Q}=\sin \alpha_{Q} H_{1}^{-Q}+\cos \alpha_{Q} H_{2}^{-Q}
\end{gathered}
$$

that is

$$
\begin{aligned}
& H_{1}^{Q}=\cos \alpha_{Q} \zeta^{Q}+\sin \alpha_{Q} \zeta^{-Q *}, \\
& H_{2}^{Q}=-\sin \alpha_{Q} \zeta^{Q}+\cos \alpha_{Q} \zeta^{-Q *},
\end{aligned}
$$

with $m_{H_{1}^{Q}}<m_{H_{2}^{Q}}$. The mixing angle, $\alpha_{Q} \in[-\pi / 2, \pi / 2]$, is then defined as

$$
\begin{aligned}
\tan \alpha_{Q} & =(-1)^{\frac{n}{2}+Q+1} \frac{Q \lambda_{3}-\sqrt{Q^{2} \lambda_{3}^{2}+\left(n^{2}-4 Q^{2}\right) \lambda_{4}^{2}}}{\lambda_{4} \sqrt{n^{2}-4 Q^{2}}} \\
& =(-1)^{\frac{n}{2}+Q} \frac{\lambda_{4} \sqrt{n^{2}-4 Q^{2}}}{Q \lambda_{3}+\sqrt{Q^{2} \lambda_{3}^{2}+\left(n^{2}-4 Q^{2}\right) \lambda_{4}^{2}}} .
\end{aligned}
$$


The mass matrices for the pairs of scalars with electric charge $Q=1, \ldots, n / 2-1$ are given in the basis $\left(\zeta^{+Q}, \zeta^{-Q *}\right)$ by

$$
M_{\zeta^{ \pm Q}}^{2}=\left(\begin{array}{ll}
M^{2}+\frac{1}{8} v^{2}\left(4 \lambda_{2}-(2 Q-1) \lambda_{3}\right) & \frac{1}{4} v^{2} \lambda_{4} \sqrt{n^{2}-4 Q^{2}}(-1)^{\frac{n}{2}+Q+1} \\
\frac{1}{4} v^{2} \lambda_{4} \sqrt{n^{2}-4 Q^{2}}(-1)^{\frac{n}{2}+Q+1} & M^{2}+\frac{1}{8} v^{2}\left(4 \lambda_{2}+(2 Q+1) \lambda_{3}\right)
\end{array}\right)
$$

which we diagonalize to find the mass eigenvalues,

$$
\begin{aligned}
m_{H_{1,2}^{Q}}^{2} & =M^{2}+\frac{1}{2} v^{2}\left(\lambda_{2}+\frac{\lambda_{3}}{4} \mp \frac{1}{2} \sqrt{Q^{2} \lambda_{3}^{2}+\left(n^{2}-4 Q^{2}\right) \lambda_{4}^{2}}\right) \\
& =m_{\zeta^{0, r}}^{2}+\frac{1}{4} v^{2}\left(n(-1)^{\frac{n}{2}} \lambda_{4} \mp \sqrt{Q^{2} \lambda_{3}^{2}+\left(n^{2}-4 Q^{2}\right) \lambda_{4}^{2}}\right) .
\end{aligned}
$$

Finally, there is one unmixed state with $Q=n / 2$. Its mass squared is given by

$$
m_{\zeta^{\frac{n}{2}}}^{2}=M^{2}+\frac{1}{8} v^{2}\left[4 \lambda_{2}-(2 Q-1) \lambda_{3}\right]=m_{\zeta^{0, r}}^{2}-\frac{n}{8} v^{2}\left[\lambda_{3}+2(-1)^{\frac{n}{2}+1} \lambda_{4}\right]
$$

Since we desire a dark matter candidate, we require that the lightest member of the multiplet be stable. The $Z_{2}$ symmetry ensures this stability, but we are free to choose which member will be lightest. This will place constraints on the possible values of $M, \lambda_{2}, \lambda_{3}$, and $\lambda_{4}$. There are stringent limits on charged relics [105], and we would like to ensure that our dark matter candidate is dark (i.e. it does not interact with photons), so we choose that the neutral component of $\Sigma$ is the dark matter candidate. This requires that $\left|\lambda_{3}\right|<2\left|\lambda_{4}\right|$. Since $\lambda_{4}$ splits $\zeta^{0}$ into two physical states, we could choose either $\zeta^{0, r}$ or $\zeta^{0, i}$ to be the lightest (and, hence, our DM candidate). Without loss of generality, we pick $\zeta^{0, r}$. This then sets $\lambda_{4}^{(6)}<0$ and $\lambda_{4}^{(8)}>0$. 

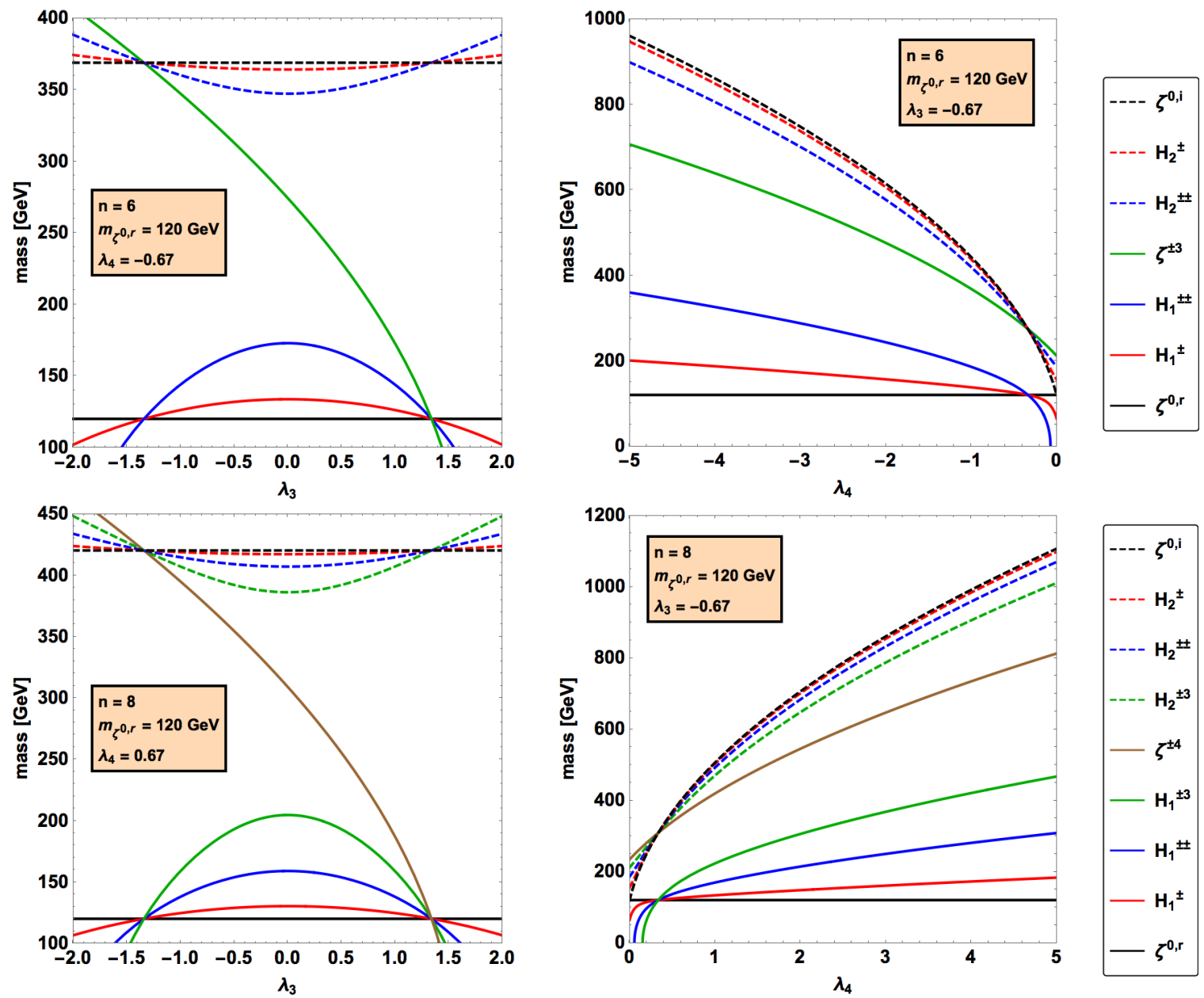

Figure 3.1. Mass vs. $\lambda_{3}$ (left-hand plots) and $\lambda_{4}$ (right-hand plots) for $n=6$ (top plots) and $n=8$ (bottom plots). In these plots, the value of either $\lambda_{3}$ or $\lambda_{4}$ is held constant (as labelled in the inset box) and the other is varied.

The physical scalars arising from the large multiplet occur in the following mass hierarchy. In the region of interest, $\left|\lambda_{3}\right|<2\left|\lambda_{4}\right|$, from lightest to heaviest,

$$
\begin{aligned}
& \Sigma_{(n=6)} \rightarrow \zeta^{0, r}, H_{1}^{ \pm}, H_{1}^{ \pm \pm}, \zeta^{+3}, H_{2}^{ \pm \pm}, H_{2}^{ \pm}, \zeta^{0, i} \\
& \Sigma_{(n=8)} \rightarrow \zeta^{0, r}, H_{1}^{ \pm}, H_{1}^{ \pm \pm}, H_{1}^{ \pm 3}, \zeta^{+4}, H_{2}^{ \pm 3}, H_{2}^{ \pm \pm}, H_{2}^{ \pm}, \zeta^{0, i}
\end{aligned}
$$

Sample spectra for various values of $n, m_{\zeta^{0, r}}, \lambda_{3}$, and $\lambda_{4}$ are shown in Figure 3.1. 
Recall that $\left|\lambda_{3}\right|<2\left|\lambda_{4}\right|$ for $\zeta^{0, r}$ to be the lightest state. One of the constraints which will derive in Chapter 4 will show that $\lambda_{3} \simeq-2\left|\lambda_{4}\right|$ unless $\lambda_{3}$ and $\lambda_{4}$ are quite small. This leads to a clustering of the mass eigenstates in two groups: $\left\{\zeta^{0, r}, H_{1}^{Q}\right\}$ and $\left\{\zeta^{\frac{n}{2}}, H_{2}^{Q}, \zeta^{0, i}\right\}$, where $Q=1, \ldots, \frac{n}{2}-1$. In the limit $\lambda_{3}=-2\left|\lambda_{4}\right|$, these clusters each have degenerate mass. This can be most easily seen in the mass vs. $-\lambda_{3}$ plots of Figure 3.1.

From Equation (3.4), we know that the states of $\Sigma$ interact with the Standard Model through a coupling to the Higgs doublet as well as through the gauge-kinetic terms. Due to the $Z_{2}$ symmetry, all couplings will involve two members of $\Sigma$, but may include either one or two SM states. The full list of couplings is given in Appendix A. The couplings to the Higgs boson and the photon are diagonal (these couplings involve a state from $\Sigma$ and its anti-particle) and so cannot induce decays. However, the couplings to the $W$ and $Z$ bosons have off-diagonal elements. Decays of heavier states in $\Sigma$ will thus involve emission of a $W$ or $Z$ which may be on- or off-shell. Examples of spectra showing mass versus charge are given in Figure 3.2 for various parameter points. The vertical and diagonal arrows in these plots show decays from the heavier states to the lighter states. Solid lines indicate that the decay is on-shell, while dashed lines indicate an off-shell decay.

\subsection{Re-parameterization of the models}

For the LHC direct search constraints we will detail in Section 5.3, it is more convenient to describe the parameter space in terms of the physical mass differences $\Delta m_{0}$ and $\Delta m_{\frac{n}{2}}$, since these splittings are monotonic in $-\lambda_{3}$ and $\lambda_{4}$ respectively. The 

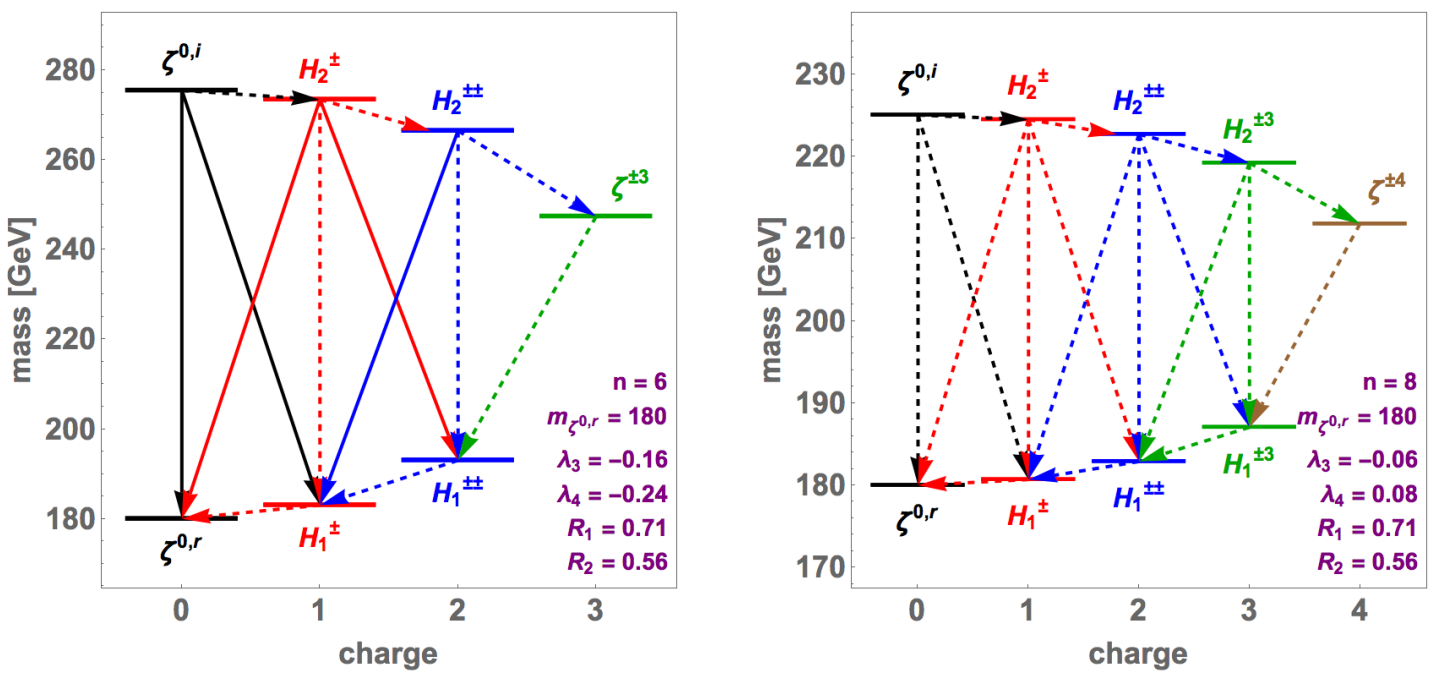

Figure 3.2. Mass vs. charge plots for $m_{\zeta^{0, r}}=180 \mathrm{GeV}$ in both $n=6$, 8. In these plots, the solid vertical and diagonal lines represent possible decays where the emitted particle is on-shell, while the dashed vertical and diagonal lines represent decays where the emitted particle is offshell.

mass splittings are given by

$$
\begin{aligned}
& \Delta m_{0} \equiv m_{\zeta^{0, i}}-m_{\zeta^{0, r}}=\sqrt{m_{\zeta^{0, r}}^{2}+\frac{n}{2}(-1)^{\frac{n}{2}} v^{2} \lambda_{4}}-m_{\zeta^{0, r}}, \\
& \Delta m_{\frac{n}{2}} \equiv m_{\zeta^{+\frac{n}{2}}}-m_{\zeta^{0, r}}=\sqrt{m_{\zeta^{0, r}}^{2}-\frac{n}{8} v^{2}\left[\lambda_{3}+2(-1)^{\frac{n}{2}+1} \lambda_{4}\right]}-m_{\zeta^{0, r}} .
\end{aligned}
$$

We define normalized versions of these splittings, each lying in the range [0, 1], as

$$
\begin{aligned}
R_{1} & \equiv \frac{\Delta m_{\frac{n}{2}}}{\Delta m_{0}}=\frac{m_{\zeta^{+\frac{n}{2}}}-m_{\zeta^{0, r}}}{m_{\zeta^{0, i}}-m_{\zeta^{0, r}}} \\
R_{2} & \equiv \frac{\Delta m_{0}}{\Delta m_{0}^{\mathrm{MAX}}}=\frac{m_{\zeta^{0, i}}-m_{\zeta^{0, r}}}{\left(m_{\zeta^{0, i}}-m_{\zeta^{0, r}}\right)^{\mathrm{MAX}}} .
\end{aligned}
$$


The relations in Equations (3.16) and (3.17) can be inverted to obtain $\lambda_{3}$ and $\lambda_{4}$ as follows:

$$
\begin{aligned}
& \lambda_{3}=\frac{4 R_{2} \Delta m_{0}^{\mathrm{MAX}}}{n v^{2}}\left[2\left(1-2 R_{1}\right) m_{\zeta^{0, r}}+\left(1-2 R_{1}^{2}\right) R_{2} \Delta m_{0}^{\mathrm{MAX}}\right], \\
& \lambda_{4}=(-1)^{\frac{n}{2}} \frac{2 R_{2} \Delta m_{0}^{\mathrm{MAX}}}{n v^{2}}\left[2 m_{\zeta^{0, r}}+R_{2} \Delta m_{0}^{\mathrm{MAX}}\right] .
\end{aligned}
$$

Notice as $R_{1} \rightarrow 0$, it is clear that $\lambda_{3} \rightarrow+2\left|\lambda_{4}\right|$, while as $R_{1} \rightarrow 1, \lambda_{3} \rightarrow-2\left|\lambda_{4}\right|$. As seen in Figure 3.1, the mass splittings are maximized when $R_{1} \sim 0.5\left(\right.$ or $\lambda_{3} \sim 0$ ), while the spectrum collapses into two clusters when $R_{1} \rightarrow 0$, or 1 . Furthermore, since $R_{2}$ describes the splitting between the two neutral states (i.e. the overall splitting in the model), when $R_{2} \rightarrow 0$ the entire spectrum becomes degenerate whereas the overall splitting is maximized when $R_{2} \rightarrow 1$.

The physical masses can also be expressed as

$$
\begin{aligned}
m_{\zeta^{0, i}} & =m_{\zeta^{0, r}}+R_{2} \Delta m_{0}^{\mathrm{MAX}} \\
m_{H_{1,2}^{+Q}} & =\sqrt{m_{\zeta^{0, r}}^{2}+\frac{1}{2} R_{2}^{2}\left(\Delta m_{0}^{\mathrm{MAX}}\right)^{2}+R_{2}\left[m_{\zeta^{0, r}} \mp \frac{\mathbb{M}}{2 n}\right] \Delta m_{0}^{\mathrm{MAX}}} \\
m_{\zeta^{+\frac{n}{2}}} & =m_{\zeta^{0, r}}+R_{1} R_{2} \Delta m_{0}^{\mathrm{MAX}}
\end{aligned}
$$

where

$$
\begin{aligned}
& \mathbb{M}^{2}=\left(n^{2}-4 Q^{2}\right)(\left.2 m_{\zeta^{0, r}}+R_{2} \Delta m_{0}^{\mathrm{MAX}}\right)^{2} \\
&+4 Q^{2}\left[2\left(1-2 R_{1}\right) m_{\zeta^{0, r}}+\left(1-2 R_{1}^{2}\right) R_{2} \Delta m_{0}^{\mathrm{MAX}}\right]^{2} .
\end{aligned}
$$

The maximum value of the neutral mass splitting allowed by the theoretical and indirect experimental constraints, $\Delta m_{0}^{\mathrm{MAX}}$, will be derived in Chapters 4 and 5 . It 


\begin{tabular}{ccc}
\hline \hline & \multicolumn{2}{c}{$\Delta m_{0}^{\mathrm{MAX}}[\mathrm{GeV}]$} \\
$m_{\zeta^{0, r}}[\mathrm{GeV}]$ & $(n=6)$ & $(n=8)$ \\
\hline 80 & 76.98 & 39.29 \\
120 & 114.38 & 55.75 \\
180 & 170.48 & 80.43 \\
300 & 282.68 & 129.81 \\
\hline \hline
\end{tabular}

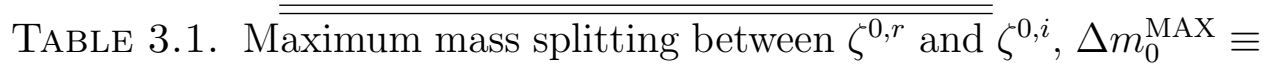
$m_{\zeta^{0, i}}-m_{\zeta^{0, r}}$, for particular values of $m_{\zeta^{0, r}}$ in the two models. Values were obtained numerically by applying the theoretical and indirect experimental constraints described in Sections 4.3 and 5.4 .

can be written parametrically for the $n=6$ and $n=8$ models as

$$
\begin{gathered}
\Delta m_{0}^{\operatorname{MAX}(n=6)} / \mathrm{GeV}= \begin{cases}2.2+0.94\left(m_{\zeta^{0, r}} / \mathrm{GeV}\right) & m_{\zeta^{0, r}} \leq 530 \mathrm{GeV} \\
809.4-0.72\left(m_{\zeta^{0, r}} / \mathrm{GeV}\right)+0.00024\left(m_{\zeta^{0, r}} / \mathrm{GeV}\right)^{2} & m_{\zeta^{0, r}}>530 \mathrm{GeV}\end{cases} \\
\Delta m_{0}^{\operatorname{MAX}(n=8)} / \mathrm{GeV}=\left\{\begin{array}{ll}
6.37+0.41\left(m_{\zeta^{0, r}} / \mathrm{GeV}\right) & m_{\zeta^{0, r}} \leq 809 \mathrm{GeV} \\
687.7-0.57\left(m_{\zeta^{0, r}} / \mathrm{GeV}\right)+0.00017\left(m_{\zeta^{0, r}} / \mathrm{GeV}\right)^{2} & m_{\zeta^{0, r}}>809 \mathrm{GeV}
\end{array} .\right.
\end{gathered}
$$

The values for a variety of mass points is given in Table 3.1. As will be seen in Section 5.4, the limit for $n=6$ (8) below $530 \mathrm{GeV}(809 \mathrm{GeV})$ comes mainly from the electroweak observables $S T U$, while above the given mass the limit comes from unitarity on the $\lambda_{i}$ parameters.

We show in Figures 3.3 and 3.4 lines of constant $\lambda_{3}$ (solid red) and $\lambda_{4}$ (dashed blue) in the $R_{1}-R_{2}$ plane for four values of $m_{\zeta^{0, r}}$. The $m_{\zeta^{0, r}}$ values chosen here reflect the choices made for the analysis in Section 5.3. Notice that although the same curves appear in each of the plots, the corresponding $\lambda_{3}$ and $\lambda_{4}$ values vary widely. 

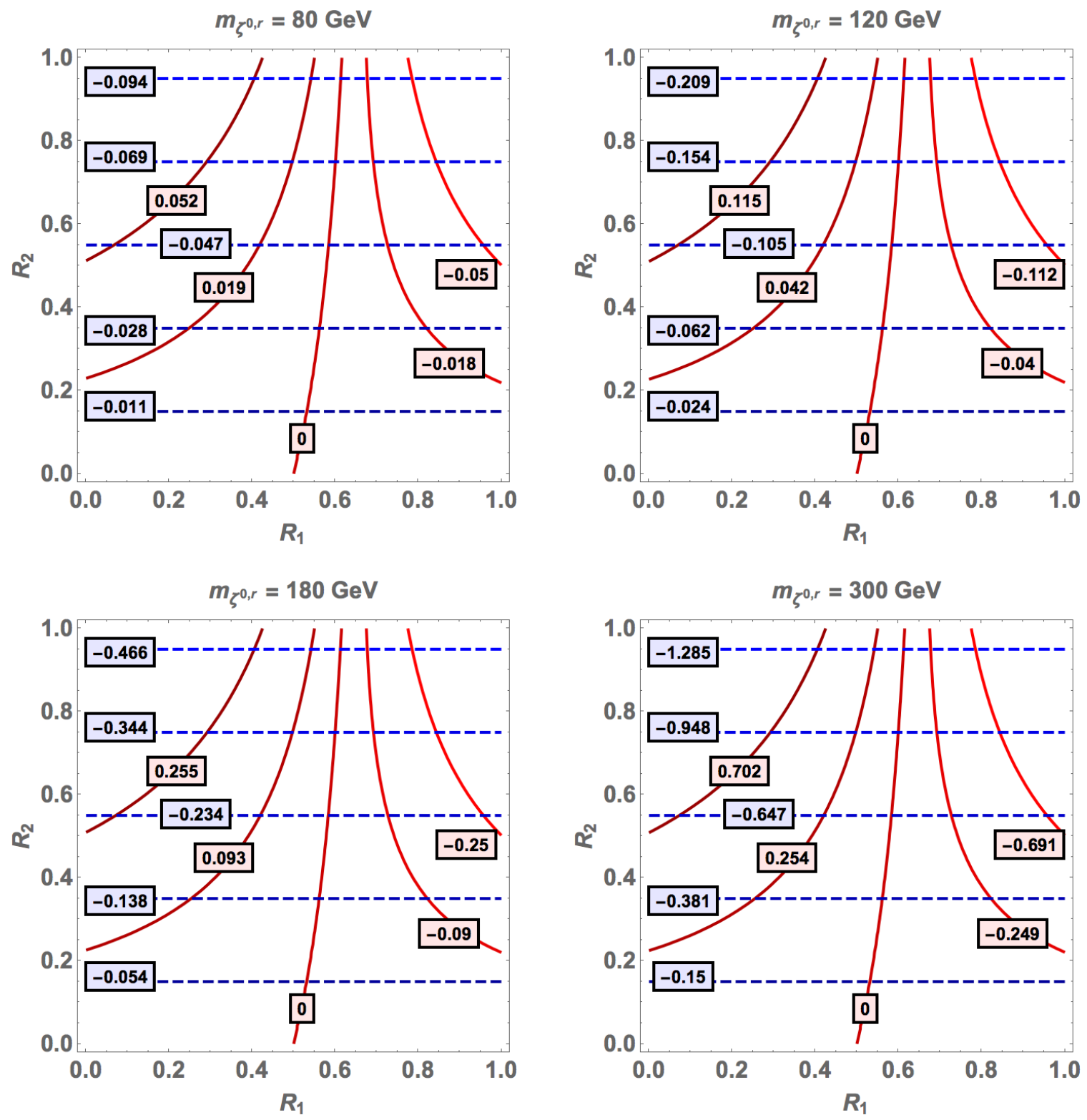

Figure 3.3. Plots showing the translation between $\lambda_{3,4}$ and $R_{1,2}$ for $n=6$. Curves of constant $\lambda_{3}$ are shown in solid red, while curves of constant $\lambda_{4}$ are shown in dashed blue. Each curve is labelled with the given value of $\lambda_{3,4}$. 

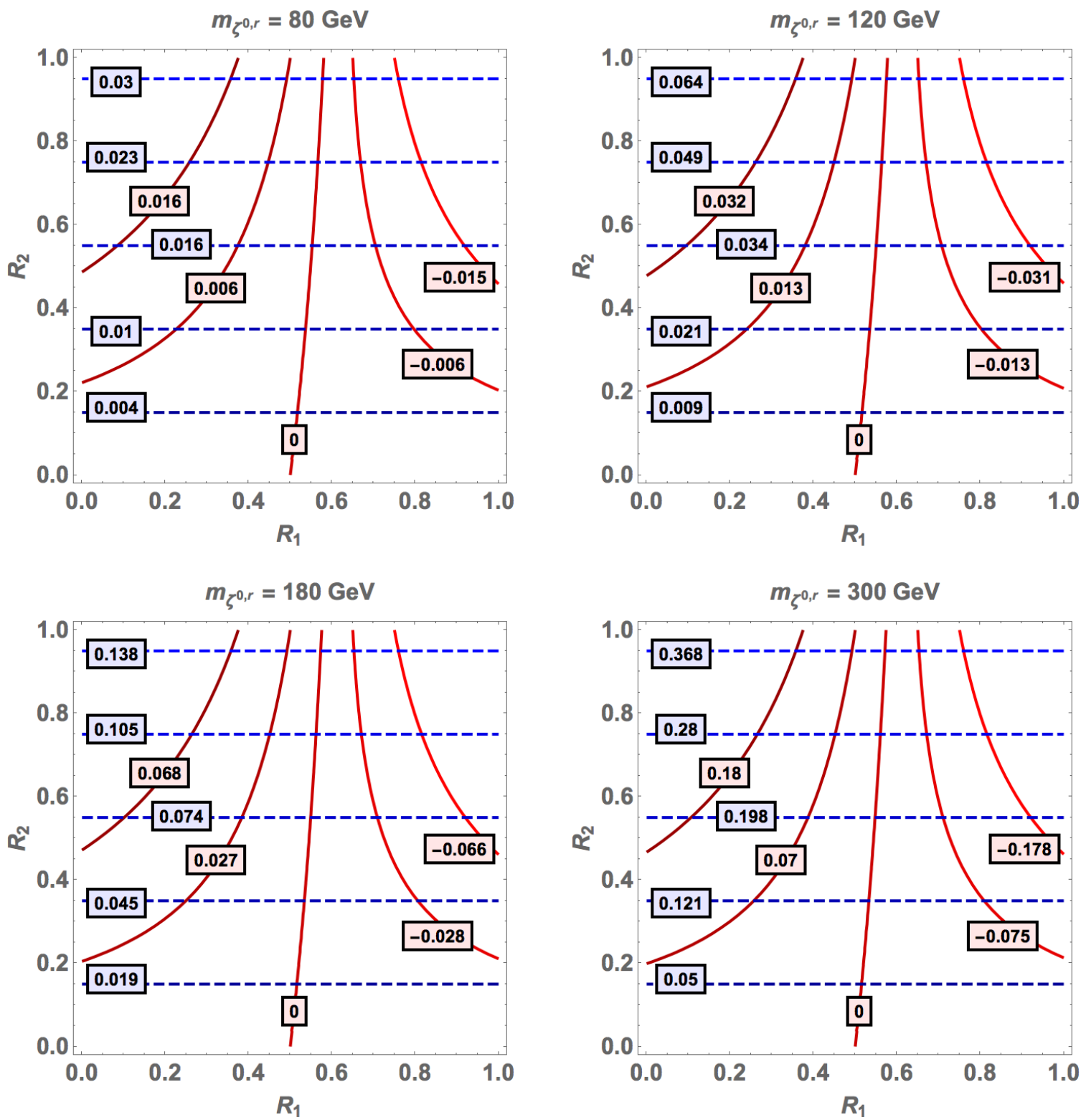

Figure 3.4. Plots showing the translation between $\lambda_{3,4}$ and $R_{1,2}$ for $n=8$. Curves of constant $\lambda_{3}$ are shown in solid red, while curves of constant $\lambda_{4}$ are shown in dashed blue. Each curve is labelled with the given value of $\lambda_{3,4}$.

\subsection{Branching ratios and preferred decay modes}

The mass splittings and coupling strengths of the scalars in the models will vary substantially over the $R_{1}-R_{2}$ plane. From Figure 3.2, we see that knowing a priori 

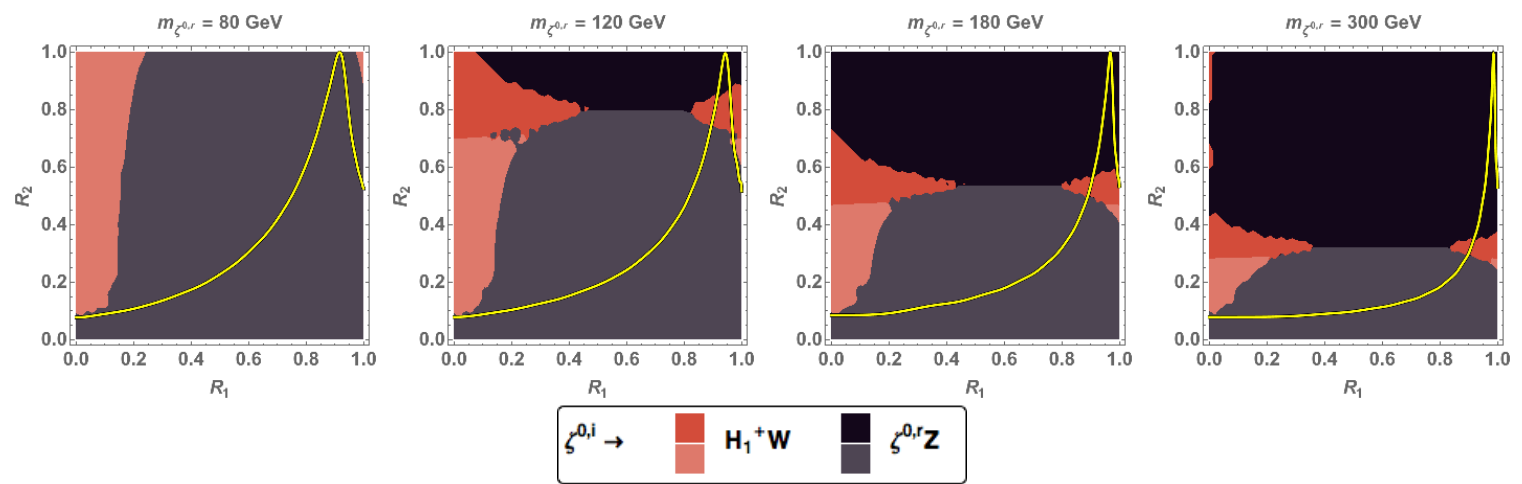

Figure 3.5. Dominant decay modes of $\zeta^{0, i}$ in the $n=6$ model. Shown are $\zeta^{0, i} \rightarrow H_{1}^{ \pm} W^{\mp}$ (orange/red) and $\zeta^{0, i} \rightarrow \zeta^{0, r} Z$ (purple/black). The decay $\zeta^{0, i} \rightarrow H_{2}^{ \pm} W^{\mp}$ is severely kinematically suppressed over the entire parameter space and never dominates. The darker shade of each colour indicates that the gauge boson is emitted on-shell, while the lighter shade indicates it is off-shell. The region below the yellow curve is allowed by indirect and precision electroweak constraints.

whether an emitted gauge boson is on- or off-shell can be complicated-although $R_{1}$ is the same in both cases, the mass splitting in the $n=6$ (left-hand plot) is sufficient to allow some on-shell decays. Furthermore, states such as $\zeta^{0, i}$ and the charged $H_{2}^{Q}$, will have three or four possible decays. Which decay will occur will affect the collider phenomenology. It is then advantageous to examine the branching ratios of these unstable states more closely. In this section, we describe the primary decay modes of the higher-mass states over the full $R_{1}-R_{2}$ plane for $m_{\zeta^{0, r}}=80,120,180$, and $300 \mathrm{GeV}$ (the mass points of interest for the collider study of Section 5.3. Decays in the $n=6$ model are shown in Figures 3.5 to 3.7 and decays in the $n=8$ model are shown in Figures 3.8 to 3.11. We do not plot the decays of the lighter $\left(\zeta^{\frac{n}{2}}\right.$ and $\left.H_{1}^{Q}\right)$ states, as they will always decay through a $W$ boson.

The numerical results were obtained as follows. We implemented the two types of models in FeynRules (version 2.0.26) [106] and generated the corresponding Universal FeynRules Output (UFO) [107] files. We then used MadGraph5_aMC@NLO (version 

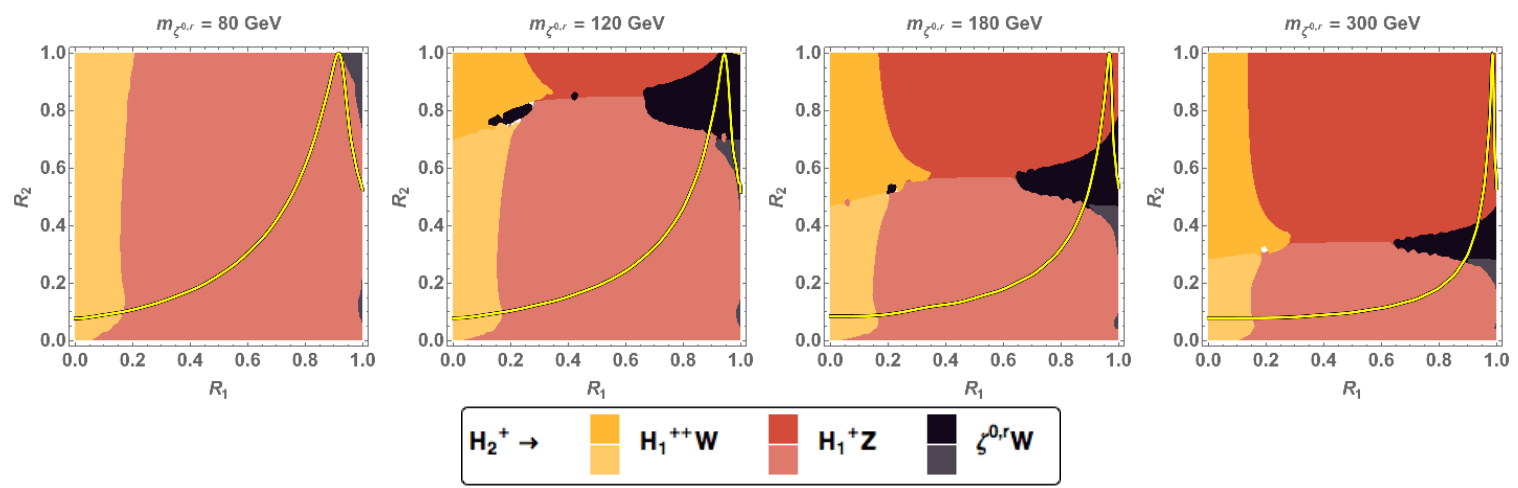

Figure 3.6. Dominant decay modes of $H_{2}^{+}$in the $n=6$ model. Shown are $\mathrm{H}_{2}^{+} \rightarrow \mathrm{H}_{1}^{++} \mathrm{W}^{-}$(yellow), $\mathrm{H}_{2}^{+} \rightarrow \mathrm{H}_{1}^{+} \mathrm{Z}$ (orange/red), and $H_{2}^{+} \rightarrow \zeta^{0, r} W^{+}$(purple/black). The decay $H_{2}^{+} \rightarrow H_{2}^{++} W^{-}$is severely kinematically suppressed over the entire parameter space and never dominates. The darker shade of each colour indicates that the gauge boson is emitted on-shell, while the lighter shade indicates it is off-shell. The region below the yellow curve is allowed by indirect and precision electroweak constraints.
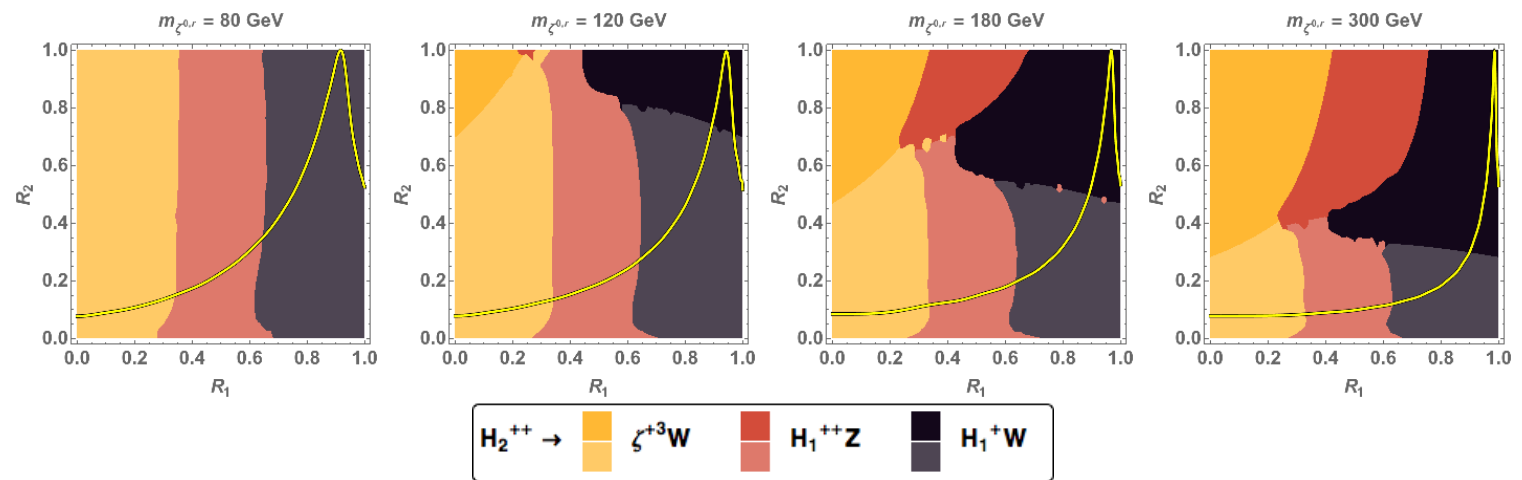

Figure 3.7. Dominant decay modes of $H_{2}^{++}$in the $n=6$ model. Shown are $H_{2}^{++} \rightarrow \zeta^{+3} W^{-}$(yellow), $H_{2}^{++} \rightarrow H_{1}^{++} Z$ (orange/red), and $\mathrm{H}_{2}^{++} \rightarrow \mathrm{H}_{1}^{+} \mathrm{W}^{+}$(purple/black). The darker shade of each colour indicates that the gauge boson is emitted on-shell, while the lighter shade indicates it is off-shell. The region below the yellow curve is allowed by indirect and precision electroweak constraints.

2.1.2) [108, 109] to automatically compute the branching ratios. This calculates the branching ratios for all necessary SM particles $(h, W, Z$, and $t)$ as well as for all new 

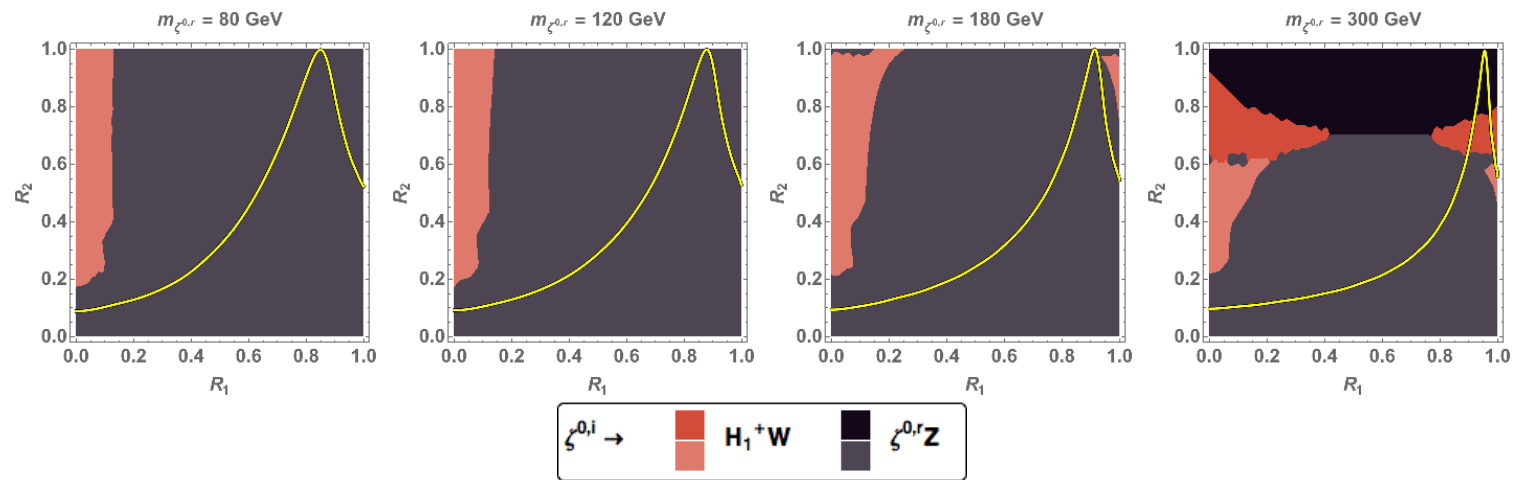

Figure 3.8. Dominant decay modes of $\zeta^{0, i}$ in the $n=8$ model. Shown are $\zeta^{0, i} \rightarrow H_{1}^{ \pm} W^{\mp}$ (orange/red) and $\zeta^{0, i} \rightarrow \zeta^{0, r} Z$ (purple/black). The decay $\zeta^{0, i} \rightarrow H_{2}^{ \pm} W^{\mp}$ is severely kinematically suppressed over the entire parameter space and never dominates. The darker shade of each colour indicates that the gauge boson is emitted on-shell, while the lighter shade indicates it is off-shell. The region below the yellow curve is allowed by indirect and precision electroweak constraints.
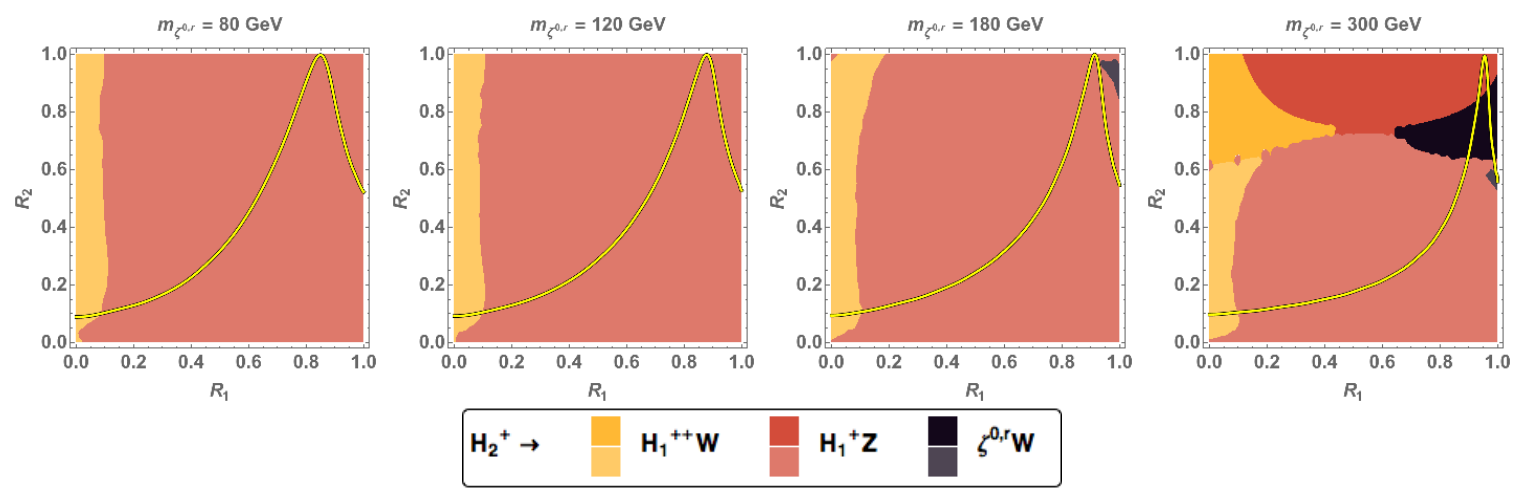

Figure 3.9. Dominant decay modes of $H_{2}^{+}$in the $n=8$ model. Shown are $H_{2}^{+} \rightarrow H_{1}^{++} W^{-}$(yellow), $H_{2}^{+} \rightarrow H_{1}^{+} Z$ (orange/red), and $\mathrm{H}_{2}^{+} \rightarrow \zeta^{0, r} W^{+}$(purple/black). The decay $\mathrm{H}_{2}^{+} \rightarrow \mathrm{H}_{2}^{++} \mathrm{W}^{-}$is severely kinematically suppressed over the entire parameter space and never dominates. The darker shade of each colour indicates that the gauge boson is emitted on-shell, while the lighter shade indicates it is off-shell. The region below the yellow curve is allowed by indirect and precision electroweak constraints.

scalars (either 2- or 3-body, depending on the mass splitting). We divided the $R_{1}-R_{2}$ plane in a grid for each of the four mass points of interest. 

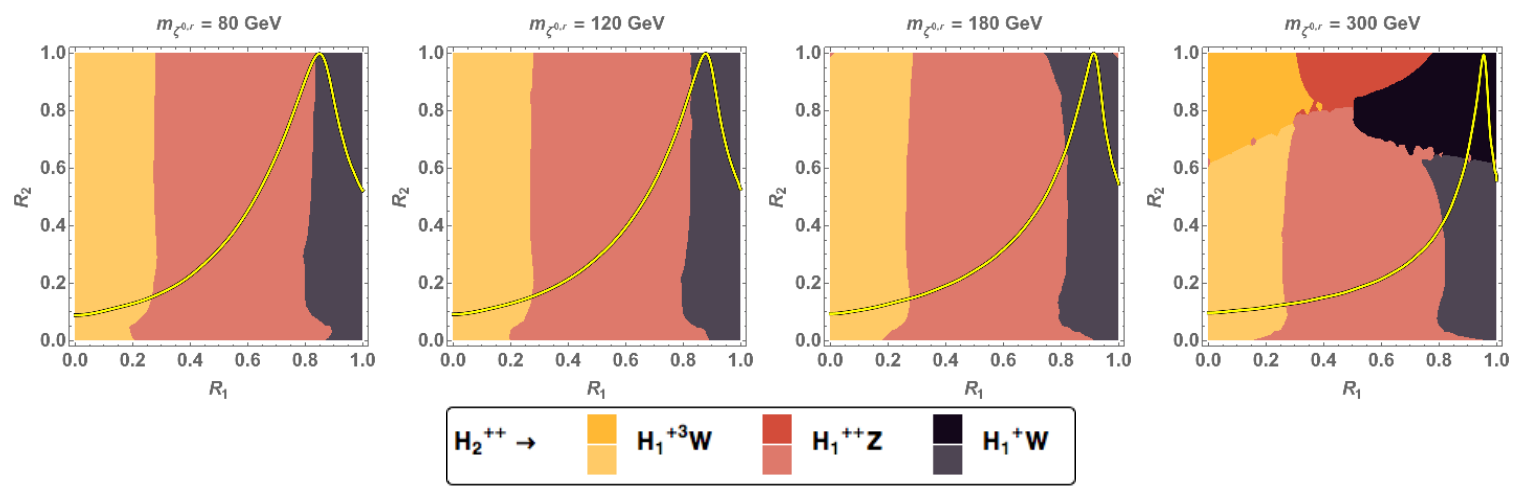

Figure 3.10. Dominant decay modes of $\mathrm{H}_{2}^{++}$in the $n=8$ model. Shown are $\mathrm{H}_{2}^{++} \rightarrow \mathrm{H}_{1}^{+3} \mathrm{~W}^{-}$(yellow), $\mathrm{H}_{2}^{++} \rightarrow \mathrm{H}_{1}^{++} \mathrm{Z}$ (orange/red), and $H_{2}^{++} \rightarrow H_{1}^{+} W^{+}$(purple/black). The decay $H_{2}^{++} \rightarrow H_{2}^{+3} W^{-}$is severely kinematically suppressed over the entire parameter space and never dominates. The darker shade of each colour indicates that the gauge boson is emitted on-shell, while the lighter shade indicates it is off-shell. The region below the yellow curve is allowed by indirect and precision electroweak constraints.
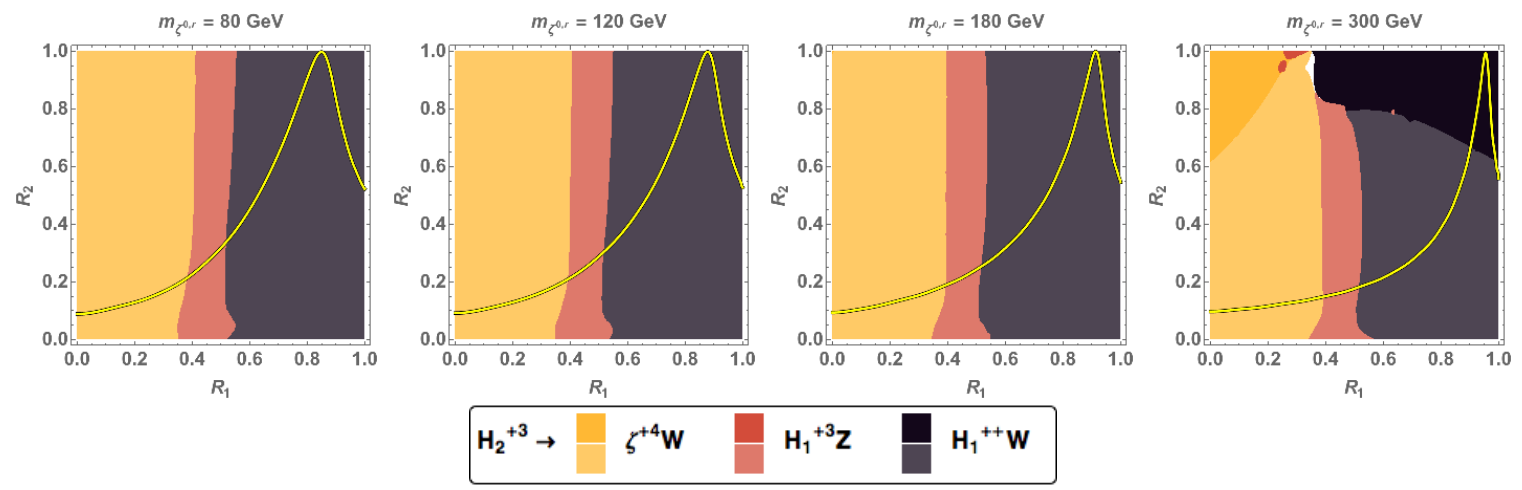

Figure 3.11. Dominant decay modes of $H_{2}^{+3}$ in the $n=8$ model. Shown are $H_{2}^{+3} \rightarrow \zeta^{+4} W^{-}$(yellow), $H_{2}^{+3} \rightarrow H_{1}^{+3} Z$ (orange/red), and $H_{2}^{+3} \rightarrow H_{1}^{++} W^{-}$(purple/black). The darker shade of each colour indicates that the gauge boson is emitted on-shell, while the lighter shade indicates it is off-shell. The region below the yellow curve is allowed by indirect and precision electroweak constraints.

The variation of the dominant branching ratio over the $R_{1}-R_{2}$ plane is due to the interplay between mass splittings and the mixing angles, $\alpha_{Q}$. The variation of the dominant decay mode with $R_{1}$ is primarily due to how the decay couplings change. 
This leads to the nearly vertical striping in Figures 3.5 to 3.11. The mass-splittingdependence appears in regions of parameter space where one or more decays are transitioning between off- and on-shell. For example, in Figure 3.7, the $H_{2}^{ \pm \pm} \rightarrow H_{1}^{ \pm} W$ goes on-shell before the decay $H_{2}^{ \pm \pm} \rightarrow H_{1}^{ \pm \pm} Z$ and so the former decay encroaches into the parameter space that would otherwise be occupied by the latter decay.

This effect also shows up in Figure 3.6. because $m_{\zeta^{0, r}}<m_{H_{1}^{++}}$, the decay of $\mathrm{H}_{2}^{+}$ to $\zeta^{0, r} W^{+}$goes on-shell while the decay to $H_{1}^{++} W^{-}$is still off-shell, leading to a small region of parameter space in which the former branching ratio surpasses the latter (the small island of black in the yellow region near the left side of the plot for $\left.m_{\zeta^{0, r}}=120 \mathrm{GeV}\right)$, despite the relative size of the couplings favouring decays to the latter 11 These features will play a role in which parts of parameter space are excluded by the LHC searches used in Section 5.3.

To illustrate the interplay of coupling strengths and mass splittings in the pattern of branching ratios, we examine in more detail two slices through the parameter space of Figures 3.7 and 3.8. In Figure 3.12 we show a slice through the $m_{\zeta^{0, r}}=180 \mathrm{GeV}$ panel of Figure 3.8 (decays of $\zeta^{0, i}$ in the $n=8$ model) at $R_{2}=0.562$. The relevant mass splittings (left plot in Figure 3.12, normalized to the mass of the daughter gauge boson) are nearly constant with $R_{1}$, as are the $\zeta^{0, i} H_{2}^{ \pm} W^{\mp}$ and $\zeta^{0, i} \zeta^{0, r} Z$ couplings (middle plot in Figure 3.12). Instead, the variation in the branching ratios is due to the variation in the $\zeta^{0, i} H_{1}^{ \pm} W^{\mp}$ coupling, which passes through zero around $R_{1} \simeq 0.6$. This behaviour can be traced back to the variation of the mixing angle $\alpha_{1}$. This results in a big dip in the $\zeta^{0, i} \rightarrow H_{1}^{ \pm} W^{\mp}$ branching ratio in favour of the decay $\zeta^{0, i} \rightarrow \zeta^{0, r} Z$ (right plot in Figure 3.12). For small $R_{1}$ where the decay to $H_{1}^{ \pm} W^{\mp}$ does dominate over $\zeta^{0, r} Z$, the $\zeta^{0, i} H_{1}^{ \pm} W^{\mp}$ coupling is still smaller than the $\zeta^{0, i} \zeta^{0, r} Z$

\footnotetext{
${ }^{1}$ The appearance of these islands depends somewhat on the grid spacing of our scan.
} 

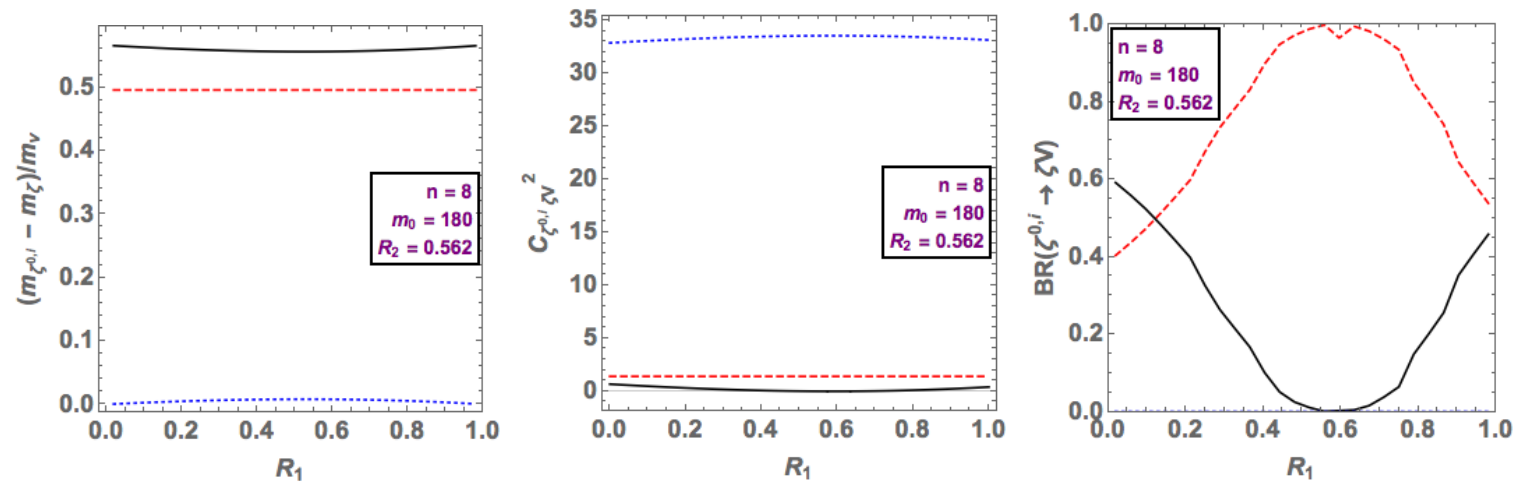

FiguRE 3.12. Scalar mass splittings normalized to the emitted gauge boson mass (left), coupling strengths squared (middle), and branching ratios (right) for $\zeta^{0, i}$ as a function of $R_{1}$ in the $n=8$ model for $m_{\zeta^{0, r}}=$ $180 \mathrm{GeV}$ and $R_{2}=0.562$ (compare Figure 3.8). Shown are the decays to $H_{1}^{ \pm} W^{\mp}$ (solid black curves), $\zeta^{0, r} Z$ (dashed red curves), and $H_{2}^{ \pm} W^{\mp}$ (dotted blue curves). Note that the $\zeta^{0, i} H_{1}^{ \pm} W^{\mp}$ squared coupling (solid black curve) touches zero near $R_{1} \simeq 0.6$.

coupling; the larger branching fraction is instead due to the larger available phase space for the former mode.

Note also that, although the $\zeta^{0, i} H_{2}^{ \pm} W^{\mp}$ coupling is significantly stronger than either of the others, the resulting decay is severely kinematically suppressed and never dominates. This is a generic feature of our models: due to the pattern of mixings among the isospin eigenstates, the gauge couplings between pairs of scalars tend to be largest when their mass splittings are smallest. The exception is the $\zeta^{0, i} \zeta^{0, r} Z$ coupling, which does not depend on any mixing angles.

In Figure 3.13 we show a slice through the $m_{\zeta^{0, r}}=120 \mathrm{GeV}$ panel of Figure 3.7 (decays of $H_{2}^{++}$in the $n=6$ model) at $R_{2}=0.429$. The three-band structure in Figure 3.7 emerges in a natural way from the interplay between couplings and mass splittings. At small $R_{1}$ the $H_{2}^{ \pm \pm} \zeta^{\mp 3} W^{ \pm}$coupling is sizeable and the available phase space is large, so this decay dominates. As $R_{1}$ increases, the mass of $\zeta^{+3}$ increases (see also Figure 3.1), and the $H_{2}^{ \pm \pm} \rightarrow \zeta^{ \pm 3} W^{\mp}$ branching fraction drops due 

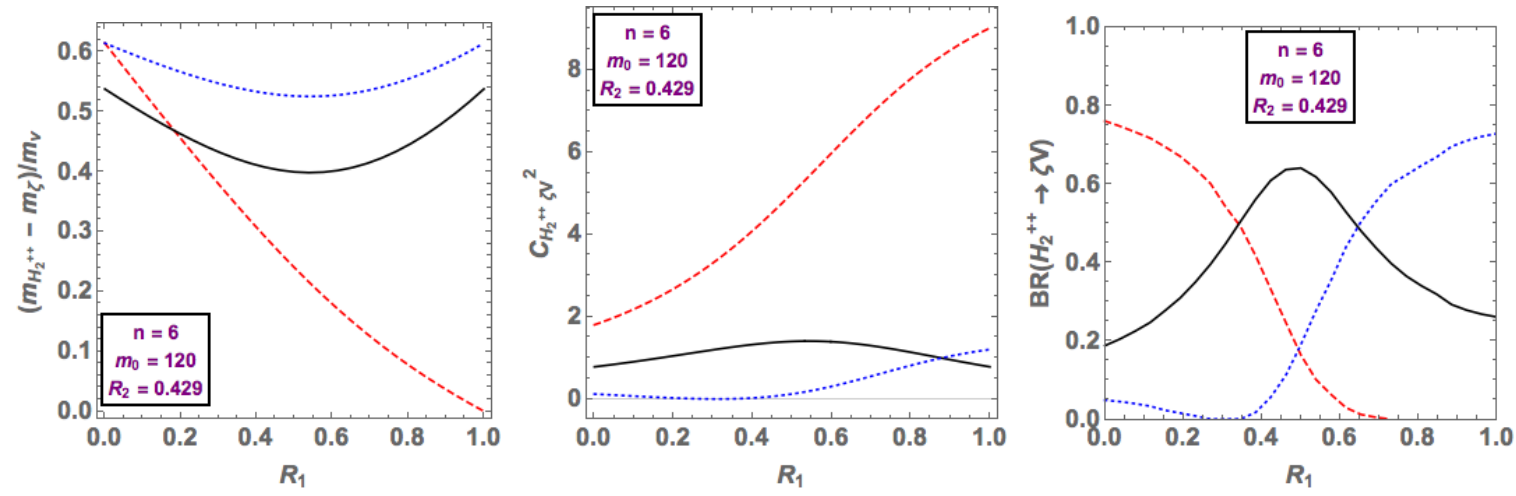

FiguRE 3.13. Scalar mass splittings normalized to the emitted gauge boson mass (left), coupling strengths squared (middle), and branching ratios (right) for $H_{2}^{++}$as a function of $R_{1}$ in the $n=6$ model for $m_{\zeta^{0, r}}=120 \mathrm{GeV}$ and $R_{2}=0.429$ (compare Figure 3.7). Shown are the decays to $\zeta^{+3} W^{-}$(dashed red curves), $H_{1}^{++} Z$ (solid black curves), and $H_{1}^{+} W^{+}$(dotted blue curves). Note that the $H_{2}^{++} H_{1}^{-} W^{-}$squared coupling (dotted blue curve) touches zero near $R_{1} \simeq 0.3$.

to the squeezing of the phase space, in spite of the growth of the $H_{2}^{ \pm \pm} \zeta^{\mp 3} W^{ \pm}$coupling. Meanwhile, the $H_{2}^{ \pm \pm} H_{1}^{\mp} W^{\mp}$ coupling is passing through zero around $R_{1} \simeq 0.3$. This allows the decay to $H_{1}^{ \pm \pm} Z$ to dominate for $R_{1}$ between about 0.3 and 0.7 . For high values of $R_{1}$, the rising $H_{2}^{ \pm \pm} H_{1}^{\mp} W^{\mp}$ coupling and its larger phase space allow the decay to $H_{1}^{ \pm} W^{ \pm}$to become dominant.

Finally, we note that the maximum allowed mass splitting $\Delta m_{0}^{\mathrm{MAX}} \equiv m_{\zeta^{0, i}}-m_{\zeta^{0, r}}$ is considerably smaller in the $n=8$ model than in the $n=6$ model (see Table 3.1). While on-shell decays first appear in the $m_{\zeta^{0, r}}=120 \mathrm{GeV}$ plots for the $n=6$ model, they only appear in the $m_{\zeta^{0, r}}=300 \mathrm{GeV}$ plots for the $n=8$ model.

\subsection{Chapter summary}

In this chapter, we detailed the large multiplet models which preserve a $Z_{2}$ symmetry of the Lagrangian. For these models, the hypercharge is specified as $Y_{\Sigma}=1$. 
We then described the scalar potential and derived the mass eigenstates. The hypercharge assignment allows to write a term (with coupling parameter $\lambda_{4}$ ) which splits the neutral member of $\Sigma$ into its real and imaginary components, and mixes the charged states into mass eigenstates $H_{k}^{Q}$. The most highly charged state, $\zeta^{\frac{n}{2}}$, is not mixed with any other state by the $\lambda_{4}$ term and thus remains a mass eigenstate. The full list of Feynman rules is quite long, and so is relegated to Appendix A.

We demand that the neutral member of the multiplet be the lightest member, which requires $\left|\lambda_{3}\right|<2\left|\lambda_{4}\right|$. We chose the real component, $\zeta^{0, r}$, to be our DM candidate, which sets a constraint of $\lambda_{4}<0$ in the $n=6$ model and $\lambda_{4}>0$ in the $n=8$ model. A re-parameterization in terms of the mass splittings $\Delta m_{0}$ and $\Delta m_{\frac{n}{2}}$ was presented. We then examined the decays of the heavier states of $\Sigma$, and showed that the dominant decay mode of each state varies considerably over the parameter space. The model description and determination of mass eigenstates was originally published in Ref. [2], and the re-parameterization and branching ratios were originally published in Ref. [3]. 


\section{CHAPTER 4}

\section{Theoretical constraints on $Z_{2}$-symmetric models}

We have used the symmetries of the Standard Model to set out the particle content and interactions of our large multiplet extension. This was done in order to furnish the SM with a viable dark matter candidate. There are, however, further considerations beyond those of Chapters 2 and 3 . As Eugene Wigner wrote [10]:

"The miracle of the appropriateness of the language of mathematics for the formulation of the laws of physics is a wonderful gift which we neither understand nor deserve. We should be grateful for it and hope that it will remain valid in future research and that it will extend, for better or for worse, to our pleasure, even though perhaps also to our bafflement, to wide branches of learning."

We make use of the wonderful mathematical tools and intuition which informed the construction of the SM and other viable extensions to constrain our large multiplet models.

In this chapter, we will first detail the source and consequences of the $Z_{2}$ symmetry imposed in Chapter 3. We will then use the requirements of stability and boundedfrom-below to constrain parameters of the scalar potential. As in Section 2.5, we will use perturbative unitarity of the scattering amplitude to place further constraints on the scalar potential parameters. Finally, we will examine the (one-loop) renormalization group equations of the large multiplet models to determine their viability up to high energy scales. 
Mass dimension

\begin{tabular}{cc}
\hline Action $(\mathcal{S})$ & 0 \\
Lagrangian $(\mathcal{L})$ & 4 \\
differential $(d x)$ & -1 \\
derivative $\left(\partial / \partial x^{\mu}\right)$ & 1 \\
mass $(m)$ & 1 \\
momentum $\left(p^{\mu}\right)$ & 1 \\
scalar $(\phi)$ & 1 \\
$\operatorname{vector}\left(A^{\mu}\right)$ & 1 \\
spinor $(\psi)$ & $3 / 2$
\end{tabular}

TABLE 4.1. Mass dimensions of common fields in four spacetime dimensions. In natural units, all quantities may be expressed in terms of one quantity. In this case, the convention we follow is energy, typically measured in $\mathrm{GeV}$. For instance, mass is given in $\mathrm{GeV}$, while length is in $\mathrm{GeV}^{-1}$, and time is also in $\mathrm{GeV}^{-1}$.

\subsection{Symmetry of the potential}

Since the action, $\mathcal{S}$, describing particle interactions is dimensionless, the terms in the Lagrangian, $\mathcal{L}$, must have dimension four, since

$$
\mathcal{S}=\int d^{4} x \mathcal{L}
$$

Any term in the Lagrangian with dimension less than four must be multiplied by a dimensionful coupling, and any term with dimension higher than four must be multiplied by a coupling with negative dimension (i.e., divided by a mass to some power such that the overall dimension of the term is four). Mass dimensions of common fields and operators are given in Table 4.1. 
The theories in which we are interested are renormalizable. That is, we would like that our theories do not give infinite results for calculations of physical values. A renormalizable theory has two types of parameters: those with positive mass dimension, and dimensionless couplings [111. This dictates the form of the terms we may include in our Lagrangians. For our models, the scalar potential involves two fields: the Standard Model Higgs doublet, $\Phi$, and the large multiplet, $\Sigma$. In general, then, we may have terms with some combination of one, two, three, or four scalar fields.

The most general scalar potential that is gauge-invariant (terms with only one or three scalar fields are not gauge invariant) is given by

$$
\begin{aligned}
V(\Phi, \Sigma) & =\mu_{h}^{2} \Phi^{\dagger} \Phi+\lambda_{1}\left(\Phi^{\dagger} \Phi\right)^{2} \\
& +M^{2} \Sigma^{\dagger} \Sigma+\lambda_{2} \Phi^{\dagger} \Phi \Sigma^{\dagger} \Sigma+\lambda_{3} \Phi^{\dagger} T_{\Phi}^{a} \Phi \Sigma^{\dagger} T_{\Sigma}^{a} \Sigma \\
& +\left(\lambda_{4} \widetilde{\Phi}^{\dagger} T_{\Phi}^{a} \Phi \Sigma^{\dagger} T_{\Sigma}^{a} \widetilde{\Sigma}+\text { h.c. }\right)+\left(\lambda_{5}[\Sigma \Sigma]_{3}^{\dagger}[\Phi \Sigma]_{3}+\text { h.c. }\right) \\
& +\lambda_{6}[\Sigma \Sigma]_{1}^{\dagger}[\Sigma \Sigma]_{1}+\lambda_{7}[\Sigma \Sigma]_{3}^{\dagger}[\Sigma \Sigma]_{3} \\
& +\lambda_{8}[\Sigma \Sigma]_{5}^{\dagger}[\Sigma \Sigma]_{5}+\delta_{n 8} \lambda_{9}[\Sigma \Sigma]_{7}^{\dagger}[\Sigma \Sigma]_{7}
\end{aligned}
$$

where the terms $[\Sigma \Sigma]_{T}$ are the properly-normalized isospin- $T$ combinations of two $\Sigma$ fields. The potential is organized in this manner to make manifest the counting of independent terms. Each valid combination of isospin and hypercharge is present. Recall that the large multiplet carries total isospin $T=\frac{n-1}{2}$ and hypercharge $Y_{\Sigma}=1$. Even-isospin combinations are zero because they would be odd under interchange of two identical $\Sigma$.

For the model to have a dark matter candidate, it must have a stable neutral particle. This means that any term with an odd number of $\Sigma$ fields would lead to 


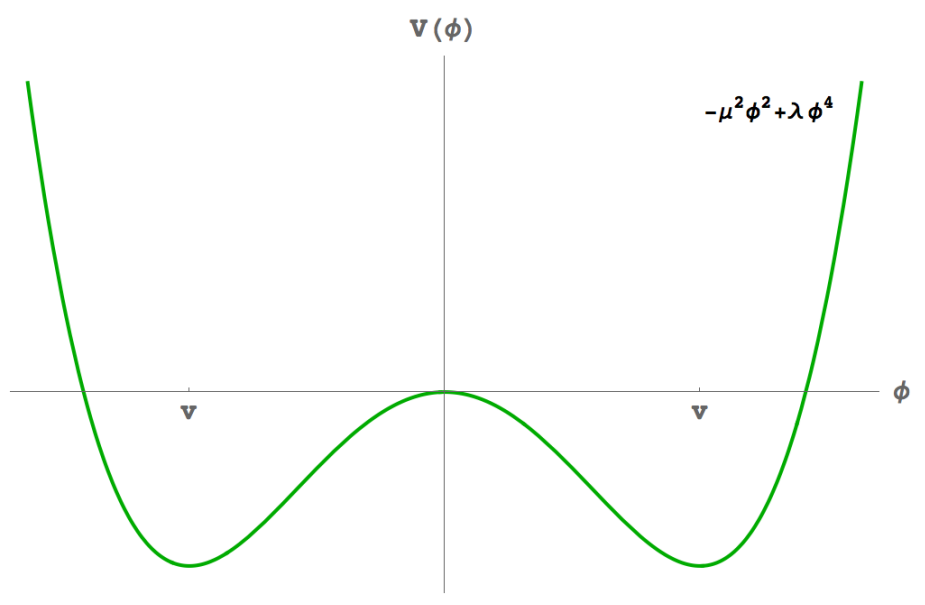

Figure 4.1. Plot of a simple quartic scalar potential, $V=-\mu^{2} \phi^{2}+$ $\lambda \phi^{4}$. In this figure, the minima occur at $\phi=v \equiv \pm^{\mu} / \sqrt{2 \lambda}$.

decays of the lightest state. Thus, we impose a $Z_{2}$ symmetry on the scalar potential under which $\Sigma \rightarrow-\Sigma$ and any SM field goes to itself. This eliminates the $\lambda_{5}$ term.

\subsection{Stability of the potential and bounded from below conditions}

We have discussed the Higgs mechanism in qualitative terms so far, but now we will provide more mathematical detail. In doing so, we will illuminate some characteristics that our scalar potential must satisfy in order that the vacuum does not become unstable.

First, we consider a very simple scalar potential involving a real singlet field, $\phi$,

$$
V=-\mu^{2} \phi^{2}+\lambda \phi^{4}
$$

for which we would like to find the extrema in $\phi$-space [112]. This is done simply by

$$
0=\frac{\partial V}{\partial \phi}=-2 \mu^{2} \phi+4 \lambda \phi^{3}=2 \phi\left(-\mu^{2}+2 \lambda \phi^{2}\right)
$$

which will have extrema at $\phi=0$ and $\phi^{2}=\mu^{2} / 2 \lambda$. We can plot this, as in Figure 4.1. 
Assuming $\mu^{2} / \lambda>0$, as we have in the plot, then $\phi^{2}=\mu^{2} / 2 \lambda$ is the global minimum of the potential, which we call the vacuum expectation value (VEV). If we add additional scalar fields to the theory, it will add further dimensions in the field space, changing the shape and thus the minima of the potential.

The situation in the SM is not as simple as that above (see, e.g., [14 for a more detailed presentation). We have measured masses for the $W$ and $Z$ bosons, but the $S U(3)_{c} \otimes S U(2)_{L} \otimes U(1)_{Y}$ gauge group predicts that they should be massless. The solution to this is the Higgs mechanism, which breaks $S U(2)_{L} \otimes U(1)_{Y}$ to $U(1)_{\mathrm{EM}}$. To make mass-generating terms for the gauge bosons, a single real scalar particle such as $\phi$ would not be sufficient since it needs to be charged under both $S U(2)_{L}$ and $U(1)_{Y}$. However, to generate masses for the fermions with a scalar field, we require a doublet of $S U(2)_{L}$, since the fermion mass term would be of the form

$$
m \bar{\psi} \psi=m \bar{\psi}_{R} \psi_{L}-m \bar{\psi}_{L} \psi_{R}
$$

which is not gauge-invariant. If we introduce a complex scalar $S U(2)_{L}$ doublet with hypercharge $Y_{\Phi}=1, \Phi=\left(\phi^{+}, \phi^{0}\right)$, and use, e.g., $L_{L}$ and $e_{R}$ from Section 2.2, we may write a gauge-invariant mass term for the electron as

$$
y_{e} \bar{e}_{R} \Phi^{\dagger} L_{L}+y_{e}^{*} \bar{L}_{L} \Phi e_{R} \rightarrow-\frac{y_{e} v}{\sqrt{2}} \bar{e} e-\frac{y_{e}}{\sqrt{2}} h \bar{e} e=-m_{e} \bar{e} e-\frac{m_{e}}{v} h \bar{e} e
$$

where we took $\phi^{0} \rightarrow(h+v) / \sqrt{2}$ (see below).

The Higgs doublet, $\Phi$, is a complex scalar field, so it can be written in terms of four real scalar fields,

$$
\Phi=\left(\begin{array}{c}
\phi^{+} \\
\phi^{0}
\end{array}\right)=\frac{1}{\sqrt{2}}\left(\begin{array}{c}
\phi_{1}+i \phi_{2} \\
\phi_{3}+i \phi_{4}
\end{array}\right)
$$


From the scalar potential,

$$
\begin{aligned}
V & =-\mu_{h}^{2} \Phi^{\dagger} \Phi+\lambda\left(\Phi^{\dagger} \Phi\right)^{2} \\
& =-\frac{1}{2} \mu_{h}^{2}\left(\phi_{1}^{2}+\phi_{2}^{2}+\phi_{3}^{2}+\phi_{4}^{2}\right)+\frac{1}{4} \lambda\left(\phi_{1}^{2}+\phi_{2}^{2}+\phi_{3}^{2}+\phi_{4}^{2}\right)^{2},
\end{aligned}
$$

we again minimize to find

$$
\left\langle\Phi^{\dagger} \Phi\right\rangle=\frac{\mu_{h}^{2}}{2 \lambda} \equiv \frac{v^{2}}{2}
$$

The VEV, $v$, is then the magnitude of $2|\Phi|^{2}=\left(\phi_{1}^{2}+\phi_{2}^{2}+\phi_{3}^{2}+\phi_{4}^{2}\right) \equiv v^{2}$. This describes a four-dimensional sphere, and anywhere on the surface of that sphere gives the minimum value. As such, the VEV may be distributed between these four components in any combination so that Equation (4.9) is satisfied. By convention, we choose that

$$
\begin{aligned}
& \left\langle\phi_{1}\right\rangle=0, \quad\left\langle\phi_{2}\right\rangle=0, \\
& \left\langle\phi_{3}\right\rangle=v, \quad\left\langle\phi_{4}\right\rangle=0 .
\end{aligned}
$$

We can further define an additional real scalar, $h$, with $\langle h\rangle=0$, so that $\phi_{3}=h+v$. The Higgs doublet then becomes

$$
\Phi=\frac{1}{\sqrt{2}}\left(\begin{array}{c}
\phi_{1}+i \phi_{2} \\
h+v+i \phi_{4}
\end{array}\right) \quad,\langle\Phi\rangle=\frac{1}{\sqrt{2}}\left(\begin{array}{l}
0 \\
v
\end{array}\right)
$$

Substituting this into the scalar potential gives us a mass term for $h$,

$$
\frac{1}{2} m_{h}^{2} \equiv \frac{1}{2}\left(3 v^{2} \lambda-\mu_{h}^{2}\right)=\frac{1}{2}\left(2 \lambda v^{2}\right)
$$

where we have used Equation (4.9) to eliminate $\mu_{h}$. The mass of $h$ is thus $m_{h}=$ $\sqrt{2 \lambda v^{2}}$. 
We have measured - through muon decay measurements of the Fermi constant, $G_{F}[\mathbf{6}$ - the VEV to be $v \approx 246 \mathrm{GeV}$. If any new fields are added to the SM which affect electroweak symmetry breaking, their contribution is limited such that the total VEV is still $\sim 246 \mathrm{GeV}$. In the two Higgs doublet models (2HDM [113; see, eg., Ref. [114] for a review), each doublet contributes, so that $v_{\Phi_{1}}^{2}+v_{\Phi_{2}}^{2}=(246 \mathrm{GeV})^{2}$, and their ratio, $v_{\Phi_{2}} / v_{\Phi_{1}} \equiv \tan \beta$ allows experiments to probe their parameter space through a single parameter.

In addition, since the $W$ and $Z$ boson masses are proportional to the VEV, any new contribution is constrained by the ratio of their masses, defined by the $\rho$-parameter,

$$
\rho \equiv \frac{m_{W}^{2}}{m_{Z}^{2} \cos ^{2} \theta_{W}}
$$

which the SM predicts to be 1 and has been measured to be $\rho=1.00040 \pm 0.00024$ [6] Isospin multiplets that carry hypercharge will contribute to the $\rho$-parameter as

$$
\rho=\frac{\sum_{i}\left[T_{i}\left(T_{i}+1\right)-Y_{i}^{2}\right] v_{i}^{2}}{2 \sum_{i} Y_{i}^{2} v_{i}^{2}},
$$

where the sum is over all fields that acquire a VEV, $T_{i}$ is the field's total isospin, $Y_{i}$ is the field's hypercharge, and $v_{i}$ is its VEV. Recall that we must still also satisfy $\sum_{i}\left[T_{i}\left(T_{i}+1\right)-Y_{i}^{2}\right] v_{i}^{2}=(246 \mathrm{GeV})^{2}$.

To ensure that the neutral component of $\Sigma$ is a DM candidate (i.e., it will not decay), we have set the tree-level VEV of the large multiplet to zero. We would expect that this should be sufficient to ensure the stability of the scalar potential. Recall that in the scalar potential, Equation (4.2), we have $V \supset M^{2} \Sigma^{2}+\widetilde{\lambda} \Sigma^{4}$ (where $\widetilde{\lambda}$ is some combination of $\lambda_{6,7,8,9}$ ). There are two possible situations that arise: $M^{2}<0$ and

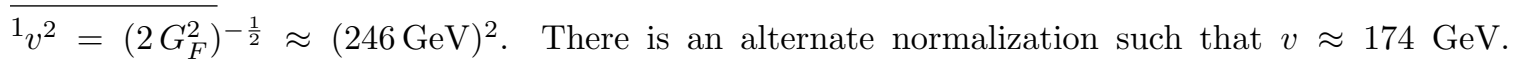
Throughout this thesis, we use $v \approx 246 \mathrm{GeV}$. 

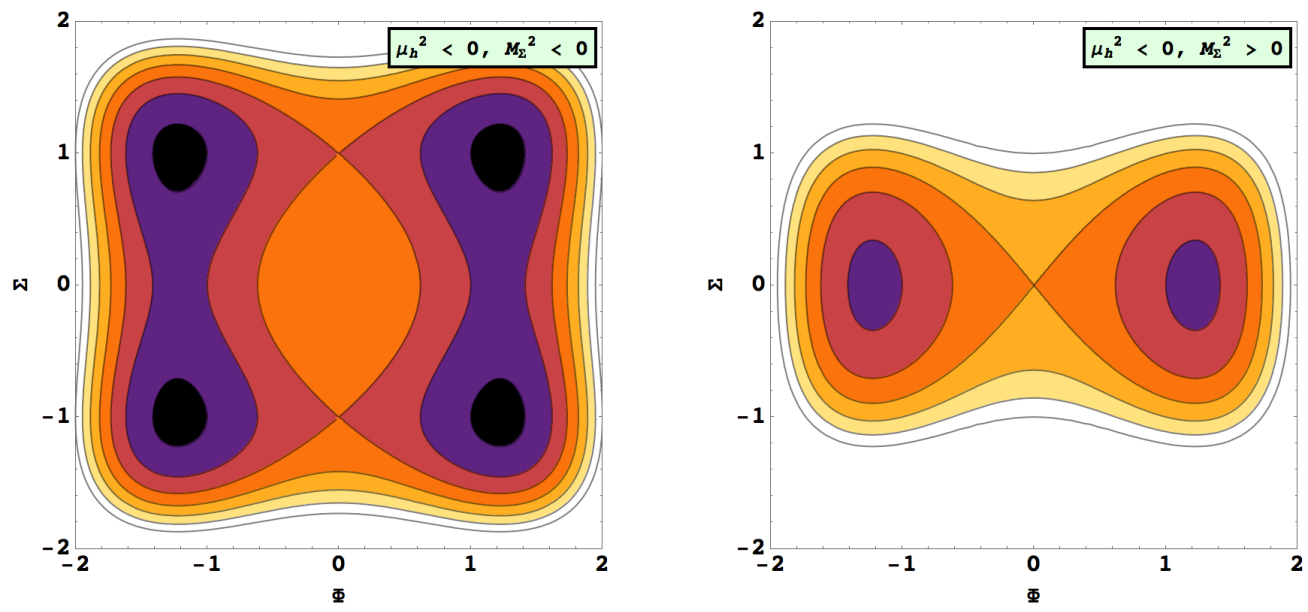

Figure 4.2. Plot of a scalar potential, $V(\Phi, \Sigma)$. The darker the colour, the deeper the potential: Black corresponds to $V<-3$, orange to $V=0$, and white to $V>2$ (the units are arbitrary). In the left-hand plot, the coefficients of both the $\Phi^{2}$ term and the $\Sigma^{2}$ term are negative. This situation leads to a deeper VEV for non-zero values of the $\Sigma$ field. In the right-hand plot, the coefficient of the $\Phi^{2}$ term is negative, but the coefficient of the $\Sigma^{2}$ term is positive. In this case, the only minima are along $\Sigma=0$ in the $\Phi$ direction. We have suppressed the three other $\Phi$ directions and $2 n-1 \Sigma$ directions.

$M^{2}>0$. If $M^{2}<0$, then the potential will dip in that direction as in the left-hand plot of Figure 4.2. The minimum of the potential is no longer at $\Sigma=0$. However, if we set $M^{2}>0$ in the scalar potential, the the only minimum will be at $\Sigma=0$ (see the right-hand plot of Figure 4.2. Thus, we choose $M^{2}>0$ in Equation (4.2).

While we have ensured that there are no alternate minima in the scalar potential, we must now consider the large $\Sigma$ behaviour. As the field values increase, the quartic terms overpower the quadratic terms and so their coefficients become important. If they are negative, then the potential may run to negative infinity - it would have no global minimum, and the vacuum would be unstable. To counter this, we must impose conditions that bound the potential from below. In the SM, there is only one quartic term, $\lambda|\Phi|^{4}$, and the potential will be bounded from below provided that 
$\lambda>0$. For our large multiplet models there are two fields, $\Phi$ and $\Sigma$, and 6 (7) quartic combinations of these fields in the $n=6$ (8) model.

We can parameterize the scalar potential in the following manner [115, 116]. Let $r^{2}=\Phi^{\dagger} \Phi+\Sigma^{\dagger} \Sigma$. Then

$$
r \cos \gamma \equiv \sqrt{\Phi^{\dagger} \Phi}, \quad r \sin \gamma \equiv \sqrt{\Sigma^{\dagger} \Sigma}
$$

and we can make the following definitions:

$$
\begin{array}{rlrl}
a & \equiv \frac{\Phi^{\dagger} T_{\Phi}^{i} \Phi \Sigma^{\dagger} T_{\Sigma}^{i} \Sigma}{\left(\Phi^{\dagger} \Phi\right)\left(\Sigma^{\dagger} \Sigma\right)}, & b \equiv \frac{\widetilde{\Phi}^{\dagger} T_{\Phi}^{i} \Phi \Sigma^{\dagger} T_{\Sigma}^{i} \widetilde{\Sigma}}{\left(\Phi^{\dagger} \Phi\right)\left(\Sigma^{\dagger} \Sigma\right)}, \\
c \equiv \frac{[\Sigma \Sigma]_{3}^{\dagger}[\Phi \Sigma]_{3}}{\left(\Phi^{\dagger} \Phi\right)^{1 / 2}\left(\Sigma^{\dagger} \Sigma\right)^{3 / 2}}, & d \equiv \frac{[\Sigma \Sigma]_{1}^{\dagger}[\Sigma \Sigma]_{1}}{\left(\Sigma^{\dagger} \Sigma\right)^{2}}, \\
e \equiv \frac{[\Sigma \Sigma]_{3}^{\dagger}[\Sigma \Sigma]_{3}}{\left(\Sigma^{\dagger} \Sigma\right)^{2}}, & f \equiv \frac{[\Sigma \Sigma]_{5}^{\dagger}[\Sigma \Sigma]_{5}}{\left(\Sigma^{\dagger} \Sigma\right)^{2}}, \\
g & \equiv \frac{[\Sigma \Sigma]_{7}^{\dagger}[\Sigma \Sigma]_{7}}{\left(\Sigma^{\dagger} \Sigma\right)^{2}} . &
\end{array}
$$

Recall that our potential, Equation (4.2), must satisfy a $Z_{2}$ symmetry, so the coefficient of the $c$ term will vanish. We can then rewrite the quartic terms of the potential of Equation (4.2) as

$$
\begin{aligned}
V^{(4)}=\frac{r^{4}}{\left(1+\tan ^{2} \gamma\right)^{2}}\left\{\lambda_{1}+\right. & {\left[\lambda_{2}+a \lambda_{3}+2 \operatorname{Re}\left(b \lambda_{4}\right)\right] \tan ^{2} \gamma } \\
& \left.+\left[d \lambda_{6}+e \lambda_{7}+f \lambda_{8}+g \lambda_{9} \delta_{n 8}\right] \tan ^{4} \gamma\right\} .
\end{aligned}
$$

The potential is bounded from below if $V^{(4)}$ is always positive. The expression in brackets is of the form $a_{1}+a_{2} y^{2}+a_{3} y^{4}$. If we would like this to be greater than zero 
for all values of $y \equiv \tan \gamma$, then

$$
a_{1}>0, \quad a_{3}>0, \quad a_{2}+2 \sqrt{a_{1} a_{3}}>0,
$$

leading to three conditions which must be satisfied for all possible field configurations,

$$
\begin{aligned}
\lambda_{1} & >0, \\
d \lambda_{6}+e \lambda_{7}+f \lambda_{8}+g \lambda_{9} \delta_{n 8} & >0, \\
\lambda_{2}+a \lambda_{3}+2 \operatorname{Re}\left(b \lambda_{4}\right)+2 \sqrt{\lambda_{1}\left[d \lambda_{6}+e \lambda_{7}+f \lambda_{8}+g \lambda_{9} \delta_{n 8}\right]} & >0 .
\end{aligned}
$$

We must find, then, the combinations of $\lambda_{i}$ such that these always hold. If we do so, then the potential will be bounded from below, and no alternate minima will occur. To ensure that no combination of $a, b, d, e, f,(g)$ violates these inequalities for any given set of values for $\lambda_{i}$ would require a scan over an unfeasibly large number of parameters. We can, however, reduce the range of certain scan parameters (notably, $a, b$, and either $f$ or $g$ ).

First, notice that

$$
\left(\Sigma^{\dagger} \Sigma\right)^{2}=[\Sigma \Sigma]_{1}^{\dagger}[\Sigma \Sigma]_{1}+[\Sigma \Sigma]_{3}^{\dagger}[\Sigma \Sigma]_{3}+[\Sigma \Sigma]_{5}^{\dagger}[\Sigma \Sigma]_{5}+\delta_{n 8}[\Sigma \Sigma]_{7}^{\dagger}[\Sigma \Sigma]_{7}
$$

Dividing both sides by $\left(\Sigma^{\dagger} \Sigma\right)^{2}$, we have

$$
1=d+e+f+\delta_{n 8} g
$$

For $n=6$, we can then get rid of $f$ in favour of $f=1-d-e$. Similarly, in $n=8$, $g=1-d-e-f$. 
Next, we can determine the range of values that the $a$ and $b$ terms take. Let $\Phi$ acquire its VEV, then

$$
\Phi^{\dagger} \Phi=\frac{v^{2}}{2} \quad, \quad \Phi^{\dagger} T_{\Phi}^{i} \Phi=-\frac{\delta_{i 3}}{2} \frac{v^{2}}{2}=-\frac{\delta_{i 3}}{2} \Phi^{\dagger} \Phi
$$

Then,

$$
a=\frac{\Phi^{\dagger} T_{\Phi}^{i} \Phi \Sigma^{\dagger} T_{\Sigma}^{i} \Sigma}{\left(\Phi^{\dagger} \Phi\right)\left(\Sigma^{\dagger} \Sigma\right)}=\frac{-\frac{\delta_{i 3}}{2} \Phi^{\dagger} \Phi}{\Phi^{\dagger} \Phi} \frac{\Sigma^{\dagger} T_{\Sigma}^{i} \Sigma}{\Sigma^{\dagger} \Sigma}=-\frac{1}{2} \frac{\Sigma^{\dagger} T_{\Sigma}^{3} \Sigma}{\Sigma^{\dagger} \Sigma} .
$$

Now, the values of $T^{3}$ run from $-T$ to $T$, so the range of possible values for $a$ is given by

$$
a \in\left[-\frac{T}{2}, \frac{T}{2}\right]
$$

Similarly, for $b$, we need

$$
\widetilde{\Phi}^{\dagger} T_{\Phi}^{i} \Phi \Sigma^{\dagger} T_{\Sigma}^{i} \widetilde{\Sigma}=\frac{1}{2} \widetilde{\Phi}^{\dagger} T_{\Phi}^{+} \Phi \Sigma^{\dagger} T_{\Sigma}^{-} \widetilde{\Sigma}+\frac{1}{2} \widetilde{\Phi}^{\dagger} T_{\Phi}^{-} \Phi \Sigma^{\dagger} T_{\Sigma}^{+} \widetilde{\Sigma}
$$

since $\widetilde{\Phi}^{\dagger} T_{\Phi}^{3} \Phi=0$ when keeping only the VEV. From the matrices for $T^{ \pm}$, the range of values for $n=6$ will be $[-\sqrt{2}, \sqrt{5} / 2]$, and for $n=8$ will be $[-\sqrt{3}, \sqrt{15} / 2]$.

In summary, we have for $n=6$ the following finite ranges,

$$
\begin{aligned}
& a \in\left[-\frac{5}{4}, \frac{5}{4}\right], \\
& b \in[-\sqrt{2}, \sqrt{5} / 2],
\end{aligned}
$$

and the inequalities

$$
\begin{aligned}
\lambda_{1} & >0, \\
d \lambda_{6}+e \lambda_{7}+(1-d-e) \lambda_{8} & >0, \\
\lambda_{2}+a \lambda_{3}+2 \operatorname{Re}\left(b \lambda_{4}\right)+2 \sqrt{\lambda_{1}\left[d \lambda_{6}+e \lambda_{7}+(1-d-e) \lambda_{8}\right]} & >0 .
\end{aligned}
$$


For $n=8$, we have

$$
\begin{aligned}
& a \in\left[-\frac{7}{4}, \frac{7}{4}\right], \\
& b \in[-\sqrt{3}, \sqrt{15} / 2],
\end{aligned}
$$

and the inequalities

$$
\begin{aligned}
\lambda_{1} & >0, \\
d \lambda_{6}+e \lambda_{7}+f \lambda_{8}+(1-d-e-f) \lambda_{9} & >0, \\
\lambda_{2}+a \lambda_{3}+2 \operatorname{Re}\left(b \lambda_{4}\right)+2 \sqrt{\lambda_{1}\left[d \lambda_{6}+e \lambda_{7}+f \lambda_{8}+(1-d-e-f) \lambda_{9}\right]} & >0 .
\end{aligned}
$$

Thus, we have determined that a sufficient condition to avoid alternate minima of the scalar potential is to set $M^{2}>0$. To ensure that the potential is boundedfrom-below, we use the conditions in Equations (4.25) to (4.28). At this stage, an implementation of the bounded-from-below conditions still requires a high-dimensional parameterization. Further simplification of these conditions is beyond the scope of this work. Next, we turn to the $\lambda_{i}$ parameters themselves.

\subsection{Unitarity constraints}

The determination of the maximum size of the $\lambda_{i}$ couplings follows a similar coupled-channel analysis as in Section 2.5. The scalar quartic couplings $\lambda_{2}, \lambda_{3}$ and $\lambda_{4}$ given in Equation (4.2) can be bounded by requiring perturbative unitarity of the zeroth partial wave scattering amplitudes. The partial wave amplitudes are related to scattering matrix elements according to

$$
\mathcal{M}=16 \pi \sum_{J}(2 J+1) a_{J} P_{J}(\cos \theta)
$$

where $J$ is the orbital angular momentum of the final state and $P_{J}(\cos \theta)$ is the corresponding Legendre polynomial. Perturbative unitarity of the zeroth partial wave 
amplitude dictates the tree-level constraint,

$$
\left|\operatorname{Re} a_{0}\right| \leq \frac{1}{2}
$$

We perform a coupled-channel analysis for processes $\Sigma \Sigma \rightarrow \Phi \Phi$ and $\Sigma \Sigma \rightarrow V V$, where $\Sigma \Sigma$ denotes any pair of scalars from the large multiplet, $\Phi \Phi$ denotes any pair of scalars from the Higgs doublet, and $V V$ denotes any pair of transversely-polarized electroweak gauge bosons.

We work in the high-energy limit and treat the Goldstone bosons as physical particles in place of the longitudinal components of the electroweak gauge bosons. For simplicity, we further neglect contributions from the quartic coupling $\lambda_{1}$, which is known to be small now that the SM-like Higgs boson mass has been measured 2 , and from quartic couplings involving four $\Sigma$ fields in the present analysis, but will return to these at the end of the section. We find numerically that including such contributions leads to tighter constraints on $\lambda_{2}, \lambda_{3}$, and $\lambda_{4}$. As such, our bounds are conservative.

The scattering amplitudes are conveniently classified according to the total isospin and total hypercharge of the initial and final two-particle states. The relevant amplitudes for the isospin-zero, hypercharge-zero channels are [5]

$$
\begin{aligned}
a_{0}\left(\left[\Sigma^{*} \Sigma\right]_{0} \rightarrow\left[\Phi^{*} \Phi\right]_{0}\right) & =-\frac{\sqrt{n}}{8 \sqrt{2} \pi} \lambda_{2}, \\
a_{0}\left(\left[\Sigma^{*} \Sigma\right]_{0} \rightarrow[W W]_{0}\right) & =\frac{g^{2}}{16 \pi} \frac{\left(n^{2}-1\right) \sqrt{n}}{2 \sqrt{3}}, \\
a_{0}\left(\left[\Sigma^{*} \Sigma\right]_{0} \rightarrow[B B]_{0}\right) & =\frac{g^{2}}{16 \pi} \frac{s_{W}^{2}}{c_{W}^{2}} \frac{Y_{\Sigma}^{2} \sqrt{n}}{2},
\end{aligned}
$$

$\overline{{ }^{2} \lambda_{1}=m_{h}^{2} / 2 v^{2}} \approx 0.13$ 
where the $\Sigma^{*} \Sigma \rightarrow W W, B B$ amplitudes include both of the contributing transverse gauge boson polarization combinations [1]. Here $g$ is the $S U(2)_{L}$ gauge coupling and $s_{W}, c_{W} \equiv \sin \theta_{W}, \cos \theta_{W}$ are the sine and cosine of the weak mixing angle, and $Y_{\Sigma}=1$ for our models. We define the following normalized isospin-zero, hyperchargezero field combinations,

$$
\begin{aligned}
{\left[\Phi^{*} \Phi\right]_{0} } & =\frac{1}{\sqrt{2}}\left(\phi^{+} \phi^{-}+\phi^{0 *} \phi^{0}\right) \\
{\left[\Sigma^{*} \Sigma\right]_{0} } & =\frac{1}{\sqrt{n}} \sum_{Q} \zeta^{Q *} \zeta^{Q}, \\
{[W W]_{0} } & =\frac{1}{\sqrt{3}}\left(\sqrt{2} W^{+} W^{-}+\frac{1}{\sqrt{2}} W^{3} W^{3}\right), \\
{[B B]_{0} } & =\frac{1}{\sqrt{2}} B B
\end{aligned}
$$

where the sum over $Q$ runs over the $n$ isospin eigenstates in $\Sigma$ as shown in Equation (3.6), The relevant amplitudes for the isospin-one, hypercharge-zero channels are 5

$$
\begin{aligned}
& a_{0}\left(\left[\Sigma^{*} \Sigma\right]_{1} \rightarrow\left[\Phi^{*} \Phi\right]_{1}\right)=-\frac{\sqrt{n\left(n^{2}-1\right)}}{32 \sqrt{6} \pi} \lambda_{3}, \\
& a_{0}\left(\left[\Sigma^{*} \Sigma\right]_{1} \rightarrow[W B]_{1}\right)=\frac{g^{2}}{16 \pi} \frac{s_{W}}{c_{W}} \frac{Y \sqrt{n\left(n^{2}-1\right)}}{\sqrt{6}},
\end{aligned}
$$

where again the $\zeta^{*} \zeta \rightarrow W B$ amplitude includes both of the contributing transverse gauge boson polarization combinations [1]. Here we used the following normalized isospin-one, hypercharge-zero field combinations,

$$
\begin{aligned}
& {\left[\Phi^{*} \Phi\right]_{1}=\frac{1}{\sqrt{2}}\left(\phi^{+} \phi^{-}-\phi^{0 *} \phi^{0}\right)} \\
& {\left[\Sigma^{*} \Sigma\right]_{1}=\sqrt{\frac{12}{n\left(n^{2}-1\right)} \sum_{Q} \zeta^{Q *} T^{3} \zeta^{Q}}} \\
& {[W B]_{1}=W^{3} B}
\end{aligned}
$$




\begin{tabular}{c|ccccccc}
\hline \hline$n$ & $\left|\lambda_{2}\right|^{\text {MAx }}$ & $\left|\lambda_{3}\right|^{\text {MAx }}$ & $\left|\lambda_{4}\right|^{\text {MAX }}$ & $\left|\lambda_{6}\right|^{\text {MAx }}$ & $\left|\lambda_{7}\right|^{\text {MAX }}$ & $\left|\lambda_{8}\right|^{\text {MAx }}$ & $\left|\lambda_{9}\right|^{\text {MAX }}$ \\
\hline 6 & 6.59 & 8.48 & 4.25 & 25.13 & 25.13 & 25.13 & - \\
8 & 3.10 & 5.46 & 2.74 & 25.13 & 25.13 & 25.13 & 25.13 \\
\hline \hline
\end{tabular}

TABLE 4.2. Upper bounds on the scalar quartic couplings from perturbative unitarity. The values for $\lambda_{2,3,4}$ are obtained using a coupledchannel analysis as describe in the text. The values for $\lambda_{6,7,8,9}$ are identical $(8 \pi)$ since these terms have already been properly normalized.

Finally, the relevant amplitude for the isospin-one, hypercharge-two channel is

$$
a_{0}\left([\Sigma \Sigma]_{1} \rightarrow[\Phi \Phi]_{1}\right)=-\frac{\sqrt{n\left(n^{2}-1\right)}}{16 \sqrt{6} \pi} \lambda_{4}
$$

where the normalized isospin-one, hypercharge-two field combinations are

$$
\begin{aligned}
& {[\Phi \Phi]_{1}=\phi^{+} \phi^{0},} \\
& {[\Sigma \Sigma]_{1}=\sqrt{\frac{6}{n\left(n^{2}-1\right)}(-1)^{n / 2} \sum_{j=1}^{n / 2}(-1)^{j}(2 j-1) \zeta^{j} \zeta^{-j+1} .}}
\end{aligned}
$$

Finding the eigenvalues of the coupled-channel amplitude matrices and applying the constraint of Equation (4.30), we obtain the following upper bounds on $\left|\lambda_{2}\right|,\left|\lambda_{3}\right|$ and $\left|\lambda_{4}\right|:$

$$
\begin{aligned}
& \left|\lambda_{2}\right| \leq \sqrt{\frac{32 \pi^{2}}{n}-\frac{g^{4}\left(n^{2}-1\right)^{2}}{24}-\frac{g^{4} s_{W}^{4}}{8 c_{W}^{4}}}, \\
& \left|\lambda_{3}\right| \leq 2 \sqrt{\frac{384 \pi^{2}}{n\left(n^{2}-1\right)}-g^{4} \frac{s_{W}^{2}}{c_{W}^{2}}}, \\
& \left|\lambda_{4}\right| \leq 8 \pi \sqrt{\frac{6}{n\left(n^{2}-1\right)}} .
\end{aligned}
$$

Numerical values $\mathrm{S}^{3}$ for $n=6$ and 8 are given in Table 4.2 .

$\overline{{ }^{3} \text { We use } g^{2}=} 4 \pi \alpha_{\mathrm{EM}} / s_{W}^{2}, s_{W}^{2}=0.231$, and $\alpha_{\mathrm{EM}}=1 / 128$. 


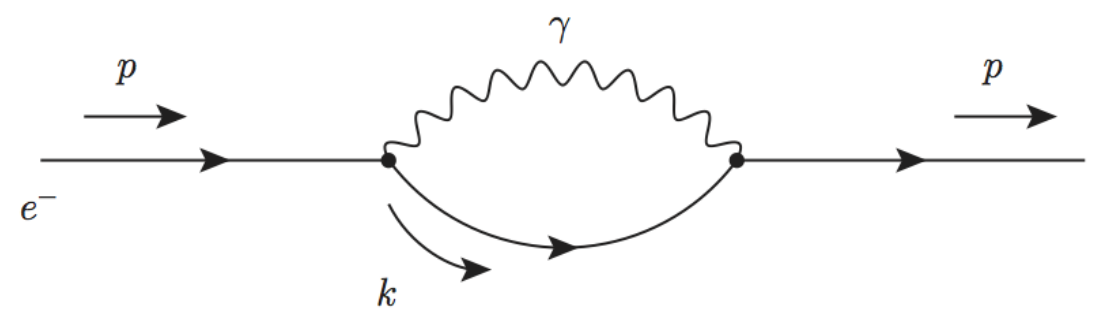

Figure 4.3. Feynman diagram for the electron propagator one-loop correction in QED.

The quartic couplings involving four $\Sigma$ fields in Equation (4.2) $-\lambda_{6}, \lambda_{7}, \lambda_{8}$, and $\lambda_{9}$ - do not need to undergo the same analysis since the combinations $[\Sigma \Sigma]_{T}^{\dagger}[\Sigma \Sigma]_{T}$ (where $T$ is the total isospin) are already properly normalized. Thus, these couplings will be constrained by

$$
\left|\lambda_{i}\right| \leq 8 \pi
$$

where we have used $\mathcal{M}=-\lambda_{i}$ for the scattering of $[\Sigma \Sigma]_{T} \rightarrow[\Sigma \Sigma]_{T}$ in Equations (4.29) and (4.30), The full form of these combinations is given in Appendix B.

\subsection{Renormalization group equations}

The parameters of a Lagrangian will receive radiative and loop corrections which may diverge at low (IR divergences) or high (UV divergences) momenta. For example, the 1-loop correction to the mass of an electron comes in the form of Figure 4.3.

The tree-level propagator for the electron is given by

$$
i \frac{\not p+m_{0}}{p^{2}-m_{0}^{2}}
$$

while the 1-loop correction to the propagator is given by $\mathbf{1 1 2}$

$$
\left(i \frac{\not p+m_{0}}{p^{2}-m_{0}^{2}}\right)\left[-i \Sigma_{2}(p)\right]\left(i \frac{\not p+m_{0}}{p^{2}-m_{0}^{2}}\right) \text {. }
$$


The two-point function, $\Sigma_{2}(p)$ accounts for integration over all possible virtual momenta, $k$. It is given by

$$
-i \Sigma_{2}(p)=(-i e)^{2} \int \frac{d^{4} k}{(2 \pi)^{4}} \gamma^{\mu} \frac{i\left(\not k+m_{0}\right)}{k^{2}-m_{0}^{2}} \gamma_{\mu} \frac{-i}{(p-k)^{2}},
$$

which diverges in both the low- $k$ (IR) and high- $k$ (UV).

The IR divergence will be cancelled by tree-level contributions from the real photon radiation, but the UV divergence remains, and increasing the number of loops will only make this divergence worse. Clearly, this is undesirable behaviour, as we know the electron's mass is finite. One way to alleviate these divergences is to cut off the integral at some definite momentum. The mass parameter will then depend implicitly on this cutoff. However, we may take the infinite part of the result and subtract it from the parameter so that the physical prediction matches the measured value at a particular energy scale. This process is known as renormalization. To relate the values of the parameters at one scale to their values at another scale, we use their renormalization group equations (RGEs).

The RGE for a parameter, $g$, is given by

$$
(4 \pi)^{2} \frac{d g}{d t}=\beta(g)
$$

where $t=\ln \mu$ is the renormalization scale, $g \equiv g(t)$ is the parameter value, and $\beta$ is some function of $g$ [112].

In the SM, we have three gauge couplings, $g_{1}, g_{2}$, and $g_{3}$, corresponding to $U(1)_{Y}$, $S U(2)_{L}$, and $S U(3)_{c}$, respectively ${ }^{4}$. If the SM were to give way to a complete theory, then we would expect that these coupling parameters unify to some common value at an energy scale where the group encompassing the SM gauge group is unbroken.

${ }^{4}$ Where $g^{\prime}=\sqrt{\frac{3}{5}} g_{1}, g=g_{2}$, and $g_{s}=g_{3}$. 


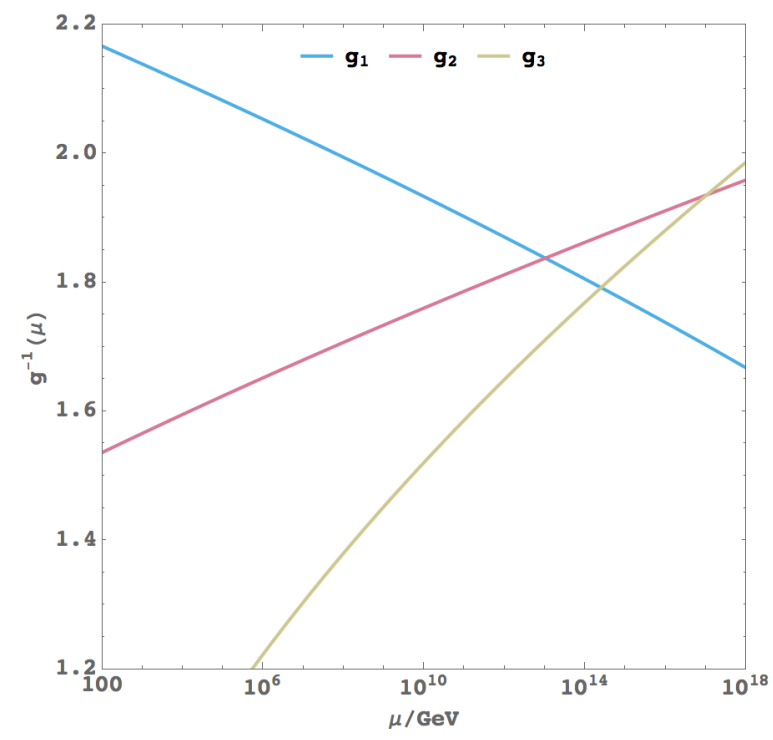

Figure 4.4. Plot of the renormalization group equation running for the SM gauge couplings. The reference scale is $\mu=m_{Z}$.

The SM one-loop RGEs for the gauge couplings are given by 93

$$
\begin{aligned}
& 16 \pi^{2} \frac{d g_{1}}{d t}=\frac{41}{10} g_{1}^{3}, \\
& 16 \pi^{2} \frac{d g_{2}}{d t}=-\frac{19}{6} g_{2}^{3}, \\
& 16 \pi^{2} \frac{d g_{3}}{d t}=-7 g_{3}^{3} .
\end{aligned}
$$

Using the values at the weak scale (measured at LEP for $\mu=m_{Z}[\mathbf{6}]$ ) as the initial conditions, $g_{1}=0.461, g_{2}=0.652$, and $g_{3}=1.22$, we solve the differential equations to get the coupling parameters as a function of the energy scale. The resulting solutions are plotted in Figure 4.4 .

To calculate RGEs for the parameters of a Lagrangian, we must first enumerate all of the diagrams that may contribute at the desired order, and then calculate the divergent parts of these diagrams (the wavefunction, charge, and vertex renormalization functions). In models that extend the SM Lagrangian, this can quickly become 
unwieldy. Thankfully, the generic forms for calculating the 1-loop RGE 5 of models that involve simple extensions of the SM by $S U(2)_{L} \otimes U(1)_{Y}$ scalars have been worked out and published in, e.g. Ref [117]. This reference gives generic expressions for an extension with a gauge group and a scalar in some representation of that group. To extend the SM with more than one gauge group, and multiple scalars, the method will need to be extended.

We would like to extend the formalism for calculating renormalization group equations given in Ref. [117] to a Lagrangian involving more than one gauge group and more than one multiplet of scalar fields. In Ref. [117], the authors consider a Lagrangian of the form

$$
\mathcal{L}=-\frac{1}{4} F_{\mu \nu}^{a} F^{a \mu \nu}+\frac{1}{2}\left(\mathcal{D}_{\mu} \phi\right)_{i}\left(\mathcal{D}^{\mu} \phi\right)_{i}+i \bar{\psi} \gamma^{\mu} \mathcal{D}_{\mu} \psi-\bar{\psi} m_{0} \psi-\bar{\psi} h_{i} \psi \phi_{i}-V(\phi)
$$

where

$$
\begin{aligned}
F_{\mu \nu}^{a} & =\partial_{\mu} A_{\nu}^{a}-\partial_{\nu} A_{\mu}^{a}-g C^{a b c} A_{\mu}^{b} A_{\nu}^{c}, \\
\left(\mathcal{D}_{\mu} \phi\right)_{i} & =\partial_{\mu} \phi_{i}+i g \theta_{i j}^{a} \phi_{j} A_{\mu}^{a}, \\
\left(\mathcal{D}_{\mu} \psi\right)_{\alpha} & =\partial_{\mu} \psi_{\alpha}+i g t_{\alpha \beta}^{a} \psi_{\beta} A_{\mu}^{a},
\end{aligned}
$$

and the relevant term from the scalar potential, $V(\phi)$, is given by

$$
V(\phi) \supset \frac{1}{4 !} f_{i j k l} \phi_{i} \phi_{j} \phi_{k} \phi_{l}
$$

where the $\phi_{i}$ are real scalar fields. Often, the potential will be written in terms of complex fields. It is then simply a matter of expanding each field into its real and ${ }^{5}$ Generically, the 1-loop corrections will be the largest, since the contribution of each loop order is suppressed by a factor $\sim 1 /(4 \pi)^{2 L}$, where $L$ is the loop order. 
imaginary parts,

$$
\begin{aligned}
\varphi_{1} & \rightarrow \frac{1}{\sqrt{2}}\left(\phi_{1}+i \phi_{2}\right), \\
\varphi_{2} & \rightarrow \frac{1}{\sqrt{2}}\left(\phi_{3}+i \phi_{4}\right), \\
\vdots & \\
\varphi_{N} & \rightarrow \frac{1}{\sqrt{2}}\left(\phi_{2 N-1}+i \phi_{2 N}\right) .
\end{aligned}
$$

The quartic coupling functions, $f_{i j k l}$, are given by

$$
f_{i j k l}=4 ! \times \frac{\text { coefficient of } \phi_{i} \phi_{j} \phi_{k} \phi_{l} \text { in } V}{\text { number of permutations of }(i j k l)} .
$$

The RGE for the quartic scalar coupling will then be given by Equation (2.8) from Ref. [117],

$$
16 \pi^{2} \frac{d f_{i j k l}}{d t}=\beta_{i j k l}
$$

where

$$
\begin{aligned}
\beta_{i j k l} \equiv & f_{i j m n} f_{m n k l}+f_{i k m n} f_{m n j l}+f_{i l m n} f_{m n j k} \\
& -12 S_{2}(S) g^{2} f_{i j k l}+3 A_{i j k l} g^{4}+8 \operatorname{Tr}\left[h_{i} h_{m}\right] f_{m j k l}-12 H_{i j k l} .
\end{aligned}
$$

The diagrams contributing to the one-loop RGEs are shown in Figure 4.5.

In Equation (4.50), the following expressions are used

$$
\begin{gathered}
A_{i j k l} \equiv\left\{\theta^{a}, \theta^{b}\right\}_{i j}\left\{\theta^{a}, \theta^{b}\right\}_{k l}+\left\{\theta^{a}, \theta^{b}\right\}_{i k}\left\{\theta^{a}, \theta^{b}\right\}_{j l}+\left\{\theta^{a}, \theta^{b}\right\}_{i l}\left\{\theta^{a}, \theta^{b}\right\}_{j k}, \\
S_{2}(S) \delta_{i j} \equiv\left[\theta^{a} \theta^{a}\right]_{i j}
\end{gathered}
$$

and

$$
H_{i j k l} \equiv \frac{1}{3 !} \operatorname{Tr}\left[h_{i} h_{j}\left\{h_{k}, h_{l}\right\}+h_{i} h_{k}\left\{h_{j}, h_{l}\right\}+h_{i} h_{l}\left\{h_{j}, h_{k}\right\}\right]
$$


where $\theta^{a}$ are the generators of the gauge group and $h_{i}$ is the Yukawa coupling of the $i$ th scalar to the fermion $\psi$.

From Equations (4.44) and (4.50), we see that this formalism assumes a single gauge group and representation for each of a single fermion and scalar. As we know in the SM, and simple extensions of the SM such as the Inert Doublet Model (IDM) [99, 118 and Georgi-Machacek model [119, 120], there are three gauge groups, $S U(3)_{c} \otimes$ $S U(2)_{L} \otimes U(1)_{Y}$, each with their own coupling $\left(g_{s}, g\right.$, and $\left.g^{\prime}\right)$ and generators $\left(\lambda^{a}, t^{a}\right.$, and $Y / 2$ ). That means that Equation (4.45) will be modified in the following way

$$
\begin{aligned}
\left(\mathcal{D}_{\mu} \phi\right)_{i} & =\partial_{\mu} \phi_{i}+i g t_{i j}^{a} \phi_{j} A_{\mu}^{a}+i g^{\prime} \frac{Y_{i}}{2} \phi_{i} B_{\mu} \\
\left(\mathcal{D}_{\mu} \psi\right)_{\alpha} & =\partial_{\mu} \psi_{\alpha}+i g t_{\alpha \beta}^{a} \psi_{\beta} A_{\mu}^{a}+i g^{\prime} \frac{Y_{\alpha}}{2} \psi_{\alpha} B_{\mu}+i g_{s} \lambda_{\alpha \beta}^{a} \psi_{\beta} G_{\mu}^{a} .
\end{aligned}
$$

This will, of course, necessitate modifying Equation (4.50), which we do as follows:

$$
\begin{aligned}
\beta_{i j k l} \equiv & f_{i j m n} f_{m n k l}+f_{i k m n} f_{m n j l}+f_{i l m n} f_{m n j k}+3 \bar{A}_{i j k l}-12 H_{i j k l} \\
& -12 S_{2}^{\prime}(S) g^{\prime 2} f_{i j k l}-12 S_{2}(S) g^{2} f_{i j k l}+8 \operatorname{Tr}\left[h_{i}^{a} h_{m}^{a}\right] f_{m j k l} .
\end{aligned}
$$

The Feynman diagrams for contributions to each term in this expression are shown in Figure 4.5. The terms quadratic in $f_{i j k l}$, Equation (4.48), correspond to the diagrams in section $(a)$.

\footnotetext{
${ }^{6}$ The gauge field strength tensor, $F_{\mu \nu}^{a}$, is unaffected by this change.
} 


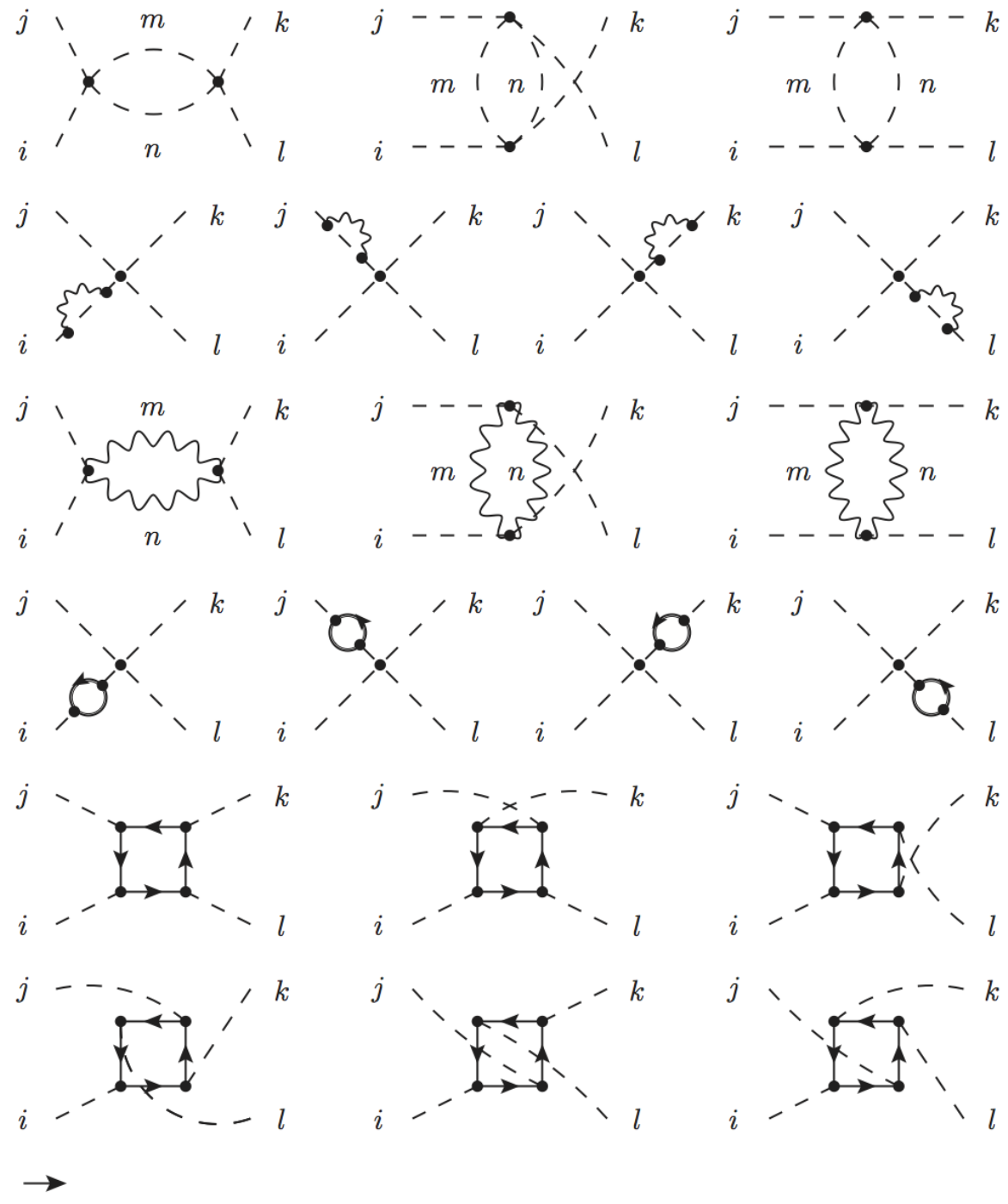

FIgURE 4.5. Feynman diagrams showing the contributions from scalar loops. Based on Figure 3 of [117].

For $U(1)_{Y}$, the generators are $Y / 2$. Then the $g^{\prime 2}$ term (section $(b)$ of Figure 4.5 with the loop representing the $B$ boson) is given by

$$
\begin{aligned}
-12 S_{2}^{\prime}(S) g^{\prime 2} & =-3 \sum_{\operatorname{legs}}\left[\theta^{a} \theta^{a}\right]_{n m} \delta_{n m} g^{\prime 2} \\
& =-3 \sum_{\operatorname{legs}}\left[\frac{Y}{2} \frac{Y}{2}\right]_{n} g^{\prime 2} \\
& =-3\left[\left(\frac{Y_{i}}{2}\right)^{2}+\left(\frac{Y_{j}}{2}\right)^{2}+\left(\frac{Y_{k}}{2}\right)^{2}+\left(\frac{Y_{l}}{2}\right)^{2}\right] g^{\prime 2},
\end{aligned}
$$


where the $i j k l$ correspond to the same indices as in Equation (4.55), and the sum is over the external leg on which the loop appears.

The coefficient of the $g^{2}$ (again, section (b) of Figure 4.5, but with the loop representing the $W^{a}$ bosons) term will have different values depending on the representation of the scalars. For $S U(2)$ doublets, each leg on which the loop is attached will have a factor

$$
\begin{aligned}
S_{2}(S)_{\text {leg }}^{\text {doublet }} \delta_{i j} & =\left[\theta^{a} \theta^{a}\right]_{i j}=\left[\sum_{a} \frac{1}{4} \sigma^{a} \sigma^{a}\right]_{i j}=\frac{1}{4}\left(\begin{array}{ll}
3 & 0 \\
0 & 3
\end{array}\right)_{i j}=\frac{3}{4} \delta_{i j} \\
& \Longrightarrow S_{2}(S)_{\text {leg }}^{\text {doublet }}=\frac{3}{4} .
\end{aligned}
$$

For $S U(2)_{L}$ triplets, each leg will have a factor of

$$
\begin{aligned}
S_{2}(S)_{\text {leg }}^{\text {triplet }} \delta_{i j} & =\left[\theta^{a} \theta^{a}\right]_{i j}=\left[\sum_{a} T^{a} T^{a}\right]_{i j}=\left(\begin{array}{lll}
2 & 0 & 0 \\
0 & 2 & 0 \\
0 & 0 & 2
\end{array}\right)_{i j}=2 \delta_{i j} \\
& \Longrightarrow S_{2}(S)_{\text {leg }}^{\text {triplet }}=2
\end{aligned}
$$

In general, the value of $S_{2}(S)_{\operatorname{leg}}$ for the $n$-dimensional representation of $S U(2)$ will be

$$
S_{2}\left[S U(2)^{(n)}\right]_{\text {leg }}=\frac{n^{2}-1}{4} .
$$

Summing over the four legs of section $(b)$ in Figure 4.5, we find

$$
\begin{aligned}
-12 S_{2}(S) g^{2} f_{i j k l} & =-3\left[S_{2}(S)_{i}+S_{2}(S)_{j}+S_{2}(S)_{k}+S_{2}(S)_{l}\right] g^{2} f_{i j k l} \\
& =-\frac{3}{4}\left(n_{i}^{2}+n_{j}^{2}+n_{k}^{2}+n_{l}^{2}-4\right) g^{2} f_{i j k l}
\end{aligned}
$$

where $n_{m}=2 T_{m}+1$ is the size of the multiplet corresponding to the $m$ th leg. 
The terms in $\bar{A}_{i j k l}$ involving $g^{4}, g^{\prime 4}$, and $g^{2} g^{\prime 2}$ (section (c) in Figure 4.5) arise from exchange of gauge bosons. The coupling of two scalars to two gauge bosons can be found by examining the anti-commutation relations between the generators of the given gauge groups. We have combined these together into the single $\bar{A}_{i j k l}$ term in Equation (4.55), We derive its form as follows. First, we redefine the generators to take the form

$$
t^{1} \equiv g T^{1}, \quad t^{2} \equiv g T^{2}, \quad t^{3} \equiv g T^{3}, \quad t^{4} \equiv g^{\prime} \frac{Y_{i}}{2} \mathbb{1}
$$

We then note that, for complex scalar multiplets $\Sigma$, containing real fields $\phi_{i}$,

$$
2 \Sigma^{\dagger}\left(t^{a} t^{b}+t^{b} t^{a}\right) \Sigma=\sum_{i, j} \phi_{i} \phi_{j} \alpha_{i j}^{a b}
$$

while for real multiplets, a factor of one-half is required for proper normalization,

$$
\Sigma^{\mathrm{T}}\left(t^{a} t^{b}+t^{b} t^{a}\right) \Sigma=\sum_{i, j} \phi_{i} \phi_{j} \alpha_{i j}^{a b}
$$

Here $a, b \in\{1,2,3,4\}, i, j \in\{1, \ldots, N\}$ for $N$ real scalar fields (e.g., for $N / 2$ complex scalars, $\left.\varphi_{1}=\left(\phi_{1}+i \phi_{2}\right) / \sqrt{2}, \varphi_{2}=\left(\phi_{3}+i \phi_{4}\right) / \sqrt{2}, \ldots, \varphi_{N / 2}=\left(\phi_{N-1}+i \phi_{N}\right) / \sqrt{2}\right)$. Notice that $\alpha_{i j}^{a b}$ is symmetric under $i \leftrightarrow j$. Thus, e.g., the coefficient of $\phi_{5} \phi_{6}$ in $\sum_{i, j} \phi_{i} \phi_{j} \alpha_{i j}^{a b}$ is $\alpha_{56}^{a b}+\alpha_{65}^{a b}$, while the coefficient of $\phi_{3} \phi_{3}$ in $\sum_{i, j} \phi_{i} \phi_{j} \alpha_{i j}^{a b}$ is $\alpha_{33}^{a b}$. So, care must be taken with factors of 2 in these terms. Then, finally,

$$
\bar{A}_{i j k l}=\sum_{a, b=1}^{4} \alpha_{i j}^{a b} \alpha_{k l}^{a b}+\alpha_{i k}^{a b} \alpha_{j l}^{a b}+\alpha_{i l}^{a b} \alpha_{k j}^{a b}
$$

For SM-like Yukawa interactions (i.e., the additional scalars do not interact with SM fermions, or interact only via mixing with the SM Higgs), we can write the Yukawa 
interaction matrices as

$$
Y_{m}^{u}=\left(\begin{array}{ccc}
y_{u} & 0 & 0 \\
0 & y_{c} & 0 \\
0 & 0 & y_{t}
\end{array}\right), Y_{m}^{d}=\left(\begin{array}{ccc}
y_{d} & 0 & 0 \\
0 & y_{s} & 0 \\
0 & 0 & y_{b}
\end{array}\right), \quad Y_{m}^{e}=\left(\begin{array}{ccc}
y_{e} & 0 & 0 \\
0 & y_{\mu} & 0 \\
0 & 0 & y_{\tau}
\end{array}\right)
$$

for $m=1,2,3,4$ (the SM Higgs fields of Equation (4.7) and

$$
Y_{m}^{q}=\left(\begin{array}{lll}
0 & 0 & 0 \\
0 & 0 & 0 \\
0 & 0 & 0
\end{array}\right)
$$

for $q \in\{u, d, e\}$ and $m>4$. The Yukawa terms of Equation (4.55) can then be written as

$$
8 \operatorname{Tr}\left[h_{i}^{a} h_{m}^{a}\right] f_{m j k l}=\left(\Upsilon_{i}+\Upsilon_{j}+\Upsilon_{k}+\Upsilon_{l}\right) f_{i j k l}
$$

where

$$
\Upsilon_{m}=\operatorname{Tr}\left[\sum_{q \in\{u, d, e\}} N_{c}^{q} Y_{m}^{q} Y_{m}^{q}\right],
$$

and $N_{c}^{q}$ is the number of colours of particle $q$. The quartic Yukawa term will be

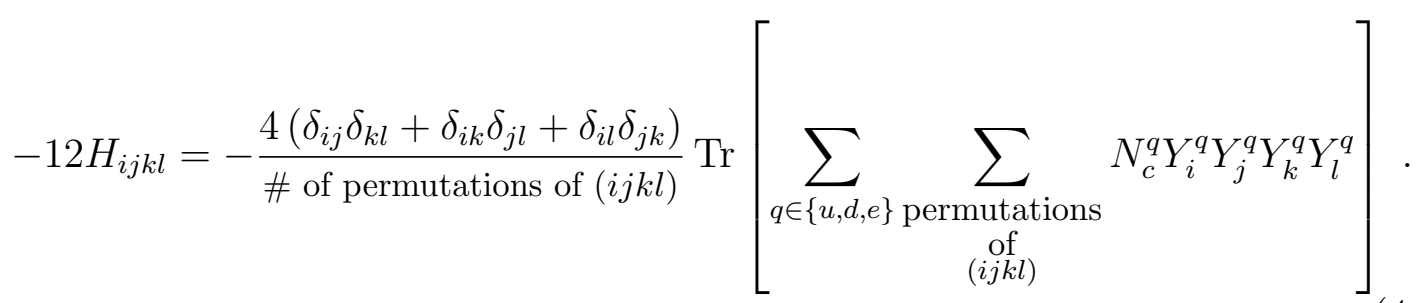

To calculate RGEs for trilinear or mass terms, we can simply append an inert real field or pair of inert real fields respectively (e.g., $\mu_{h}^{2} \Phi^{\dagger} \Phi \rightarrow \mu_{h}^{2} \phi_{0}^{2} \Phi^{\dagger} \Phi$ ) and follow the same procedure described above setting $l=0$ or $k l=00$. Since it has no interactions with any other fields, $\phi_{0}$ will have no effect on the RGEs [121. 
Substituting Equations (4.48), (4.56), (4.59), (4.63), (4.66) and (4.68) into Equation (4.55), we may now calculate the one-loop RGEs for the scalar couplings. The expressions for all of the scalar quartic couplings are given in Appendix D. We present here only one to discuss some of their main features. In the $n=6$ model, the one-loop expression for $\lambda_{7}$ is given by Equation (D.14),

$$
\begin{aligned}
16 \pi^{2} \frac{d \lambda_{7}}{d t}= & \frac{27}{200} g_{1}^{4}+\frac{297}{8} g_{2}^{4}-\frac{99}{20} g_{1}^{2} g_{2}^{2}-\frac{9}{5} g_{1}^{2} \lambda_{7}-105 g_{2}^{2} \lambda_{7}+2 \lambda_{2}^{2}-\frac{11}{8} \lambda_{3}^{2} \\
& +\frac{44}{175} \lambda_{6}^{2}+\frac{21152}{2025} \lambda_{7}^{2}+\frac{1496}{567} \lambda_{8}^{2}+\frac{4108}{1575} \lambda_{6} \lambda_{7}+\frac{44}{9} \lambda_{6} \lambda_{8}+\frac{10868}{567} \lambda_{7} \lambda_{8} .
\end{aligned}
$$

Notice that there are terms quadratic in the $\lambda_{i}$, terms quadratic in the gauge couplings times a single $\lambda_{i}$, and terms quartic in the gauge couplings. Since we know that the gauge couplings are non-zero, the scalar quartic couplings will be generated by these gauge contributions even in the case where they initially vanish. The quadratic $\lambda_{i}$ terms on the right-hand side will then provide a feedback mechanism that will cause the $\lambda_{i}$ to run. Typically, this running leads to quartic couplings diverging-leading to a so-called Landau pole - at an energy scale not too far above the weak scale.

We now determine at what scale these couplings diverge. For convenience, we numerically calculate where $\lambda^{-1}=0$. This is the scale where perturbativity of our theory breaks down - the theory becomes strongly coupled, and we cannot compute processes following the usual perturbative expansion. The results are shown in Figure 4.6. In these plots, our initial conditions are set at the weak scale, $\mu_{0}=100 \mathrm{GeV}$, 

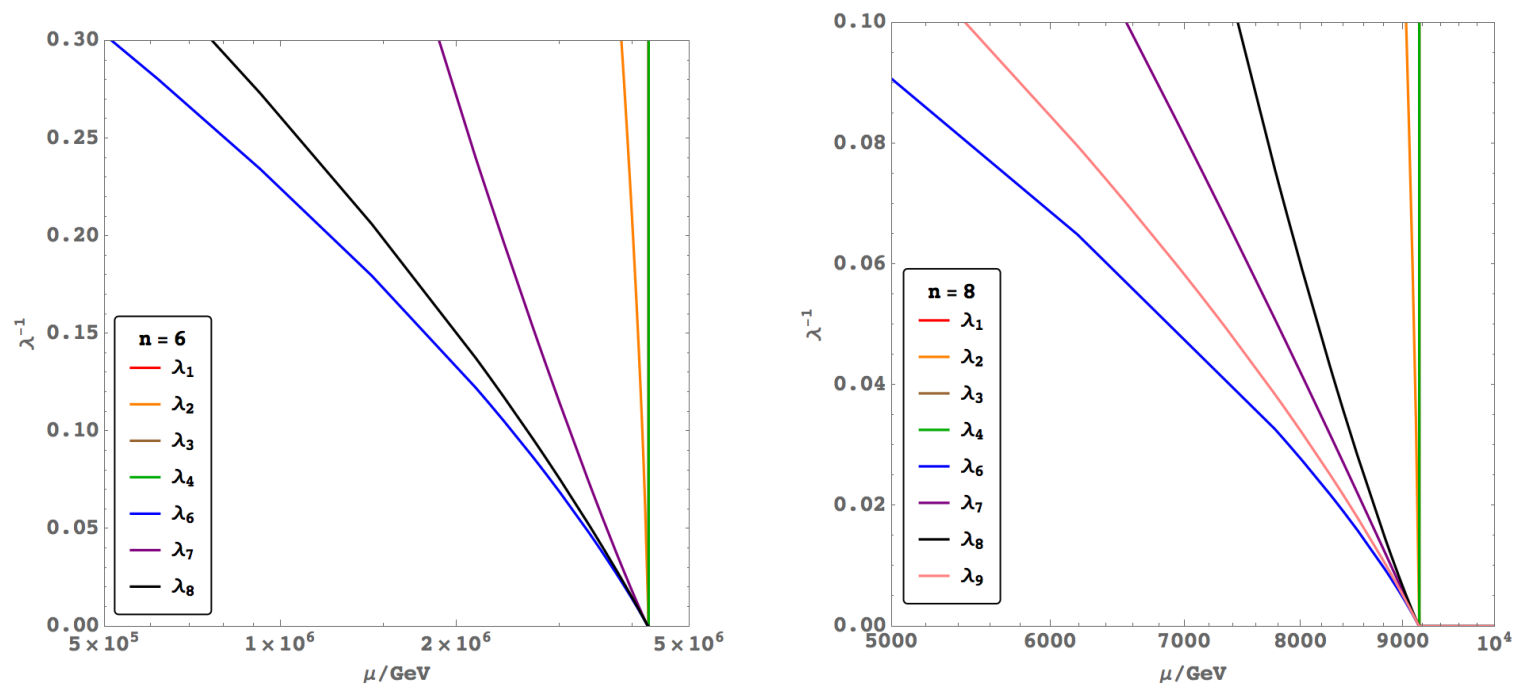

Figure 4.6. Plot showing the Landau pole location for the scalar quartic couplings of the large multiplet $n=6$ (left-hand plot) and $n=8$ (right-hand plot) models. The initial conditions in both plots are $\lambda_{1}=m_{h}^{2} / 2 v^{2}, \lambda_{i \neq 1}=0, \mu=m_{h}$, and $M=100 \mathrm{GeV}$. At this scale, the curves for $\lambda_{1}, \lambda_{3}$, and $\lambda_{4}$ overlap. The Landau pole occurs at $\mu=4.2 \times 10^{6} \mathrm{GeV}$ in $n=6$ and $\mu=9.2 \times 10^{3} \mathrm{GeV}$ in $n=8$.

and where we define the values

$$
\begin{aligned}
\lambda_{1}\left(\mu_{0}\right) & =\frac{m_{h}^{2}}{2 v^{2}}, & \lambda_{i \neq 1}\left(\mu_{0}\right) & =0, \\
g_{1}\left(\mu_{0}\right) & =\sqrt{\frac{5}{3}} \frac{\sqrt{4 \pi \alpha_{\mathrm{EM}}}}{c_{W}}, & g_{2}\left(\mu_{0}\right) & =\frac{\sqrt{4 \pi \alpha_{\mathrm{EM}}}}{s_{W}}, \\
g_{3}\left(\mu_{0}\right) & =\sqrt{4 \pi \alpha_{\mathrm{s}}}, & y_{t}\left(\mu_{0}\right) & =\sqrt{2} \frac{m_{t}}{v}, \\
\mu_{h}^{2}\left(\mu_{0}\right) & =m_{h}^{2}, & M^{2}\left(\mu_{0}\right) & =\mu_{0}^{2} .
\end{aligned}
$$

The Landau pole $\left(\lambda^{-1} \rightarrow 0\right)$ occurs at $\mu=4.2 \times 10^{6} \mathrm{GeV}$ in $n=6$ and $\mu=$ $9.2 \times 10^{3} \mathrm{GeV}$ in $n=8$. That is, they occur roughly 4 (2) orders of magnitude above the initial mass scale, $\mu_{0}=100 \mathrm{GeV}$. Despite the initial conditions above, the $\lambda_{i \neq 1}$ parameters are generated by the gauge couplings and will grow due to the feedback of 


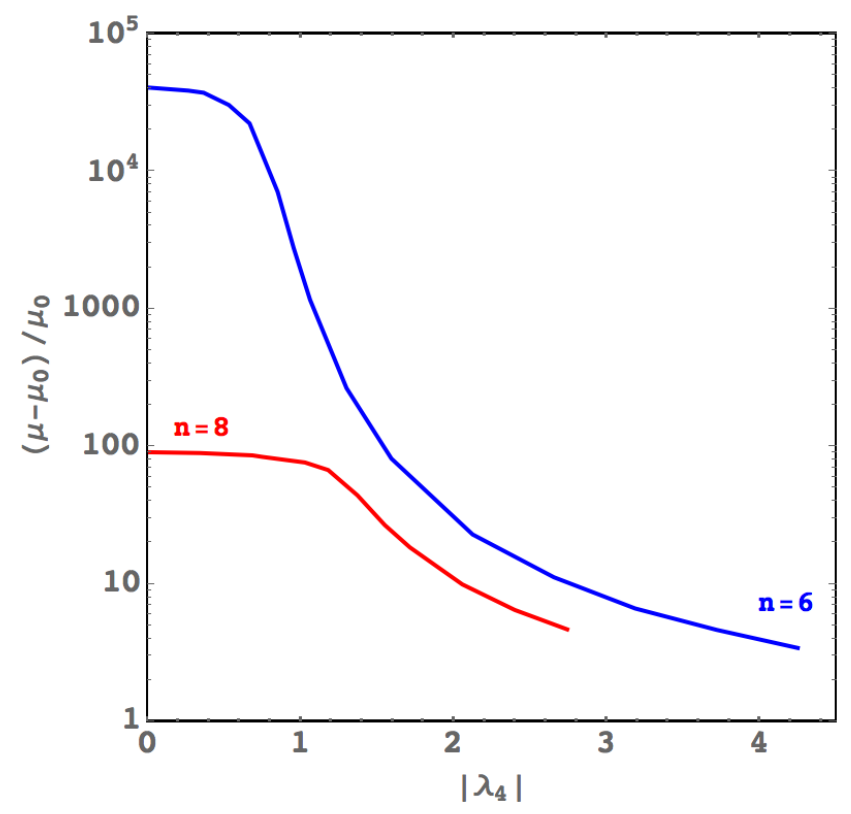

FiguRE 4.7. Normalized difference between Landau pole and initial conditions as a function of $\left|\lambda_{4}\right|$. In these figures, we set the initial conditions as in Equation (4.71). The upper blue curve corresponds to $n=6$ and the lower red curve to $n=8$. The endpoint of each curve is the unitarity bound of Section 4.3.

the $\lambda_{i}$ on the right-hand sides of the RGEs. If we set the $\lambda_{i \neq 1} \neq 0$, then the Landau pole appears at a much lower scale. This is due to the feedback of the $\lambda_{i}$ in the RGEs.

The RGEs of generic $S U(2)_{L} \otimes U(1)_{Y}$ scalars in representations of $S U(2)_{L}$ up to $n=7$ were calculated in Ref. [122] for various combinations of $n$ and $Y$. They set the same initial conditions for the $\lambda_{i \neq 1}$. The precise numerical values of the gauge couplings differ at the percent level. For $n=6$ and $Y=1$, Ref. [122] finds the Landau pole occurs at $4.6 \times 10^{6} \mathrm{GeV}$.

This scaling is relative to $M^{2}$ : recall that we have set the mass scale, $M^{2}\left(\mu_{0}\right)=\mu_{0}^{2}$, in the above. For $M \sim \mathcal{O}(10 \mathrm{TeV})$, the Landau pole will be correspondingly higher. Furthermore, if we set any of the $\lambda_{i \neq 1} \neq 0$, then the Landau pole will be significantly closer to the initial scale; the new physics must come much sooner. 
To see that this is indeed the case, in Figure 4.7, we have set the following initial conditions:

$$
\begin{aligned}
\mu_{0} & =m_{\zeta^{0, r}}, & \lambda_{1}\left(\mu_{0}\right) & =\frac{m_{h}^{2}}{2 v^{2}}, \\
\lambda_{i \neq 1,4}\left(\mu_{0}\right) & =0, & \lambda_{4}\left(\mu_{0}\right) & =\lambda_{4}, \\
g_{1}\left(\mu_{0}\right) & =\sqrt{\frac{5}{3} \frac{\sqrt{4 \pi \alpha_{\mathrm{EM}}}}{c_{W}},} & g_{2}\left(\mu_{0}\right) & =\frac{\sqrt{4 \pi \alpha_{\mathrm{EM}}}}{s_{W}}, \\
g_{3}\left(\mu_{0}\right) & =\sqrt{4 \pi \alpha_{\mathrm{s}}}, & y_{t}\left(\mu_{0}\right) & =\sqrt{2} \frac{m_{t}}{v}, \\
\mu_{h}^{2}\left(\mu_{0}\right) & =m_{h}^{2}, & M^{2}\left(\mu_{0}\right) & =\mu_{0}^{2}+\frac{1}{2} v^{2} \Lambda_{n} .
\end{aligned}
$$

We vary $m_{\zeta^{0, r}}$ for each given value of $\lambda_{4}$ so as to correspond to the correct relic abundance, which will be discussed in Section 6.4.3. This requirement is to ensure that the model has a DM candidate that accounts for all of the DM density.

We then numerically solve the RGEs to determine where $\lambda^{-1}=0$, the location of the Landau pole. We plot the difference between the Landau pole, $\mu$, and initial scale, $\mu_{0}$, normalized by $\mu_{0}$ in Figure 4.7 . In this figure, we choose $m_{\zeta^{0, r}}$ such that the correct relic abundance is achieved, as detailed in Section 6.4.3. The upper blue curve gives the ratio for $n=6$, while the lower red curve is for $n=8$. The endpoint of each curve corresponds to the unitarity bound given in Section 4.3.

We see that for relatively small quartic coupling $\left(\left|\lambda_{4}\right| \lesssim 1\right)$, the Landau pole remains roughly $4(2)$ orders of magnitude above the mass scale in the $n=6(8)$ model. As $\left|\lambda_{4}\right|$ goes beyond this value, the feedback mechanism kicks in and causes the Landau pole to occur at much lower energy; at the unitarity bound, it is less than an order of magnitude in both models. This indicates that, if our models are coupled such that $\lambda_{4}$ is near the unitarity bound, some other form of new physics must necessarily also be present. 


\subsection{Chapter summary}

In this chapter, we used the mathematical tools and intuition developed in the construction of the SM to examine our large electroweak scalar multiplet models. First, we showed how the $Z_{2}$ symmetry required for a DM candidate to be present in the models affects the type of terms we may add to the scalar potential. Next, we used the requirement of no alternate minima in the scalar potential to constrain $M^{2}>0$. We then derived a set of inequality relations (given in Equations (4.25) to (4.28) on field configurations to ensure that the potential is bounded-from-below. We used partial wave unitarity to place upper-bounds on the coefficients of the scalar quartic terms (given in Table 4.2). Finally, we examined the scaling of the scalar quartic couplings with energy, and found that, for the large multiplet at $100 \mathrm{GeV}$, the models contain a Landau pole at a scale of $\mu=4.2 \times 10^{6} \mathrm{GeV}$ in $n=6$ and $\mu=9.2 \times 10^{3} \mathrm{GeV}$ in $n=8$. The symmetry, stability, and unitarity conditions were originally published in Ref. [2]. 


\section{CHAPTER 5}

\section{Collider constraints on $Z_{2}$-symmetric models}

Mathematical requirements on a theory, while helpful, are not the only way of examining physics models. We would like our models to correspond to (at least part of) reality. In order to evaluate how well our models describe the Universe around us, we must make predictions of physical observables and perform experimental measurements. We can then compare these quantities and subsequently celebrate agreement, or go back and determine why the model failed.

For a very large number of predictions made by the SM, we fall into the former. As seen in Figure 1.2, the SM proves to be a formidable description of reality. On the other hand, we also saw how the SM is incomplete. However, since the measurements are so precise, there are few places for new physics (NP) to hide. We can use the agreement between the SM and experimental results to constrain any NP that interacts with the SM in some way. This may take one of two forms: direct searches for $\mathrm{NP}$, or through the effects of NP on SM processes. In this chapter, we will examine how direct searches for NP will constrain our $Z_{2}$-symmetric large multiplet models, and describe how our models affect SM processes at the loop level. First, we must give a brief account of how particle colliders perform their measurements and how computer software may simulate these experiments. 


\subsection{Particle colliders and event simulation}

Particle accelerators use electric fields to accelerate charged particles and steer them using magnetic fields so that they will collide in the midst of detectors so that the products of these collisions may be collected and measured. Some examples include

- Large Electron-Positron collider (LEP [123, 124]), which collided electrons and positrons;

- Hadron-Elektron Ring Anlage (HERA [125]), which collided electrons and protons;

- Super Proton Synchrotron (when using anti-protons, Sp $\bar{p} S$ [126]) and Tevatron [127], which collided protons and anti-protons; and

- Large Hadron Collider (LHC [128, 129]), which collides protons on protons or lead ions on lead ions.

These are all examples of circular colliders. The particles are accelerated in opposite directions in a ring and made to collide at specified points along the ring. There are also linear accelerators (such as SLC, the Stanford Linear Collider [130]). The constraints discussed in this chapter will come mainly from experiments at LEP and the LHC.

Once the particles have been brought together they will interact in some way and the products of this interaction will then leave the interaction point. We place detecting material around the interaction point to collect information about the products. These products may be stable - interacting with the detector until they are captured by the detector material itself - or unstable - decaying to other particles which will themselves either be stable or unstable. We see a schematic picture of a detector 

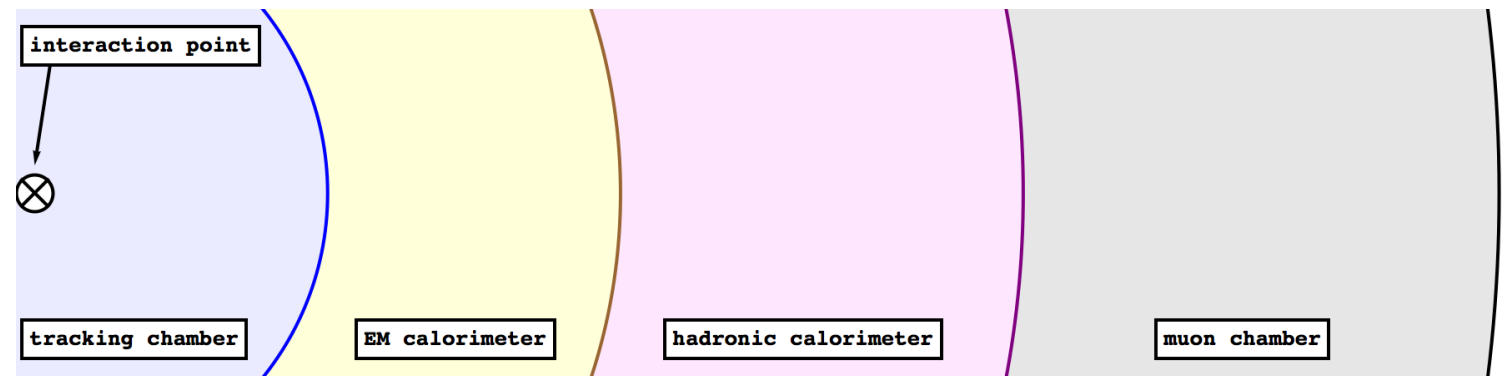

FiguRE 5.1. Simplified view of particle detectors used in collider experiments. The interaction point is in the left side of the diagram. Within the tracking chamber is a magnetic field to allow particle identification through $Q / M$ measurements. Photons and electrons will shower in the electromagnetic calorimeter, while hadrons will shower in the hadronic calorimeter. Muon identification will occur in the muon chambers. Particles such as neutrinos and dark matter will likely pass through the whole of the detector without interacting.

in Figure 5.1 [131. The sections are: (1) the interaction point; (2) the tracking chamber; (3) the electromagnetic calorimeter; (4) the hadron calorimeter; and (5) the muon chamber. These will generally be further subdivided to be able to capture more fine detail about the products of the interaction: the shape of a particle's path can tell us about its charge and momentum, or how far and wide a hadron shower is can help us identify what hadron produced the shower.

Most of the particles that a high-energy collider would produce at the interaction point (the primary vertex) will be unstable. Even through relativistic time dilation, such particles will decay almost instantaneously. To reach the innermost tracking layer of the detectors at the LHC, the remaining particles must satisfy $c \tau \gamma \gtrsim 4 \mathrm{~cm}$, where $c$ is the speed of light, $\tau$ is the particle's lifetime, and $\gamma$ is the particle's relativistic boost factor ${ }^{1}$. This reduces the number of particles we may detect to eight: leptons

\footnotetext{
${ }^{1}$ The ATLAS inner tracker is placed at $3.3 \mathrm{~cm}$ from the beam line $\mathbf{1 3 2}$. The CMS inner tracker is placed at $4 \mathrm{~cm}$ from the beam line $\mathbf{1 3 3}$.
} 
(electrons and muons), hadrons (protons, neutrons, $\pi^{ \pm}, K^{ \pm}, K_{L}^{0}$ ), and photons [134]. They will each interact differently in the various parts of the detector [131].

For example, an electron will ionize the tracking chamber as it passes through, forming a track, and then shower in the EM calorimeter. It will emit photons which will, in turn form electron-positron pairs, repeating the process. As the electron is scattered by charged particles in the detector, it will emit bremsstrahlung radiation. Through Compton scattering, photons will lose energy to electrons in the detector material. These additional (bremsstrahlung) photons and (Compton) electrons will contribute to the shower.

Gluons and quarks from the primary interaction will produce "hard" jets, while those emitted just prior to the interaction (initial-state radiation) and just after the interaction (final-state radiation) tend to form "soft," or lower-energy, jets. We know that quarks and gluons cannot exist as singular objects, since they carry colour charge, so they will form quark-antiquark pairs out of the vacuum. Through a process known as hadronization, the quarks formed in this way will combine into mesons and baryons. Adding together the energy from the particles that make up these jets will give the energy of the initial parton from the primary interaction. Energy from the hadronic shower will (mostly) be captured by the hadronic calorimeter. The hadrons interact with the detector material through the strong force, and the collisions between the hadron and a nucleus (very roughly, one nuclear collision per metre of detector material) lead to the hadronic shower.

Muons will produce tracks in the tracking chamber, but are too heavy to shower efficiently in the EM or hadronic calorimeters (they do not participate in the strong interaction, and so do not suffer the same interaction rate as hadrons). They will pass through the whole of the detector and form tracks through the muon chamber. 
Matching a track from the muon chamber with a track from the tracking chamber will provide evidence that a muon was created by the primary vertex.

Of all the quantities that detectors measure, the two of most interest in the search for $\mathrm{DM}$ are transverse momenta, $p_{T}$, and missing transverse momenta, $\mathbb{E}_{T}$ (often called missing transverse energy). Since the particles being collided at the LHC are the constituents of the protons (collectively known as partons), rather than the protons themselves, there will typically be some boost of the collision centre-of-mass system along the direction of the beam. We can account for this by making measurements transverse (perpendicular) to the direction of the beams. In that case, we measure the transverse momentum, $p_{T}$, of a particle and infer any missing momentum, $\vec{E}_{T}=$ $-\sum_{\text {visible }} \vec{p}_{T}$. We know that momentum must be conserved at every interaction, so if we measure some momenta in one direction (e.g., from the energy in a hadronic shower) with no corresponding momenta in the opposite direction to balance it, then we can infer that we have missed something. That "something" may be many lowenergy SM particles that did not trigger the detectors, SM particles that happened to pass through dead spots in a detector, very high-energy SM particles that are lost down the beam pipe, neutrinos that do not interact at all with the detector, or just maybe the DM itself.

Once all of this information has been collected we can work backwards to obtain a picture of the interaction of the initial particles. This picture is built up over many collision events and we can describe a process of interest by its interaction rate. The number of times a given final state results from a particular initial state is given by 135

$$
N=\sigma \times \int \mathcal{L} d t
$$


where $\sigma$ is the cross section of the process of interest and $\mathcal{L}$ is the instantaneous luminosity of the particle beams.

Trying to sort out what the signals mean for the SM and models beyond it is an onerous task. Experimentalists would like to find BSM physics, but each search will have unique signatures, and trying to test them all against the available data is far too time consuming. They can, however, use the most reasonable benchmark models whose signals overlap theorists' models. The theorists can do the time-consuming task of comparing the observed signals to their own particular model. How may we perform this comparison, given the complexity of high-energy collider processes? Thankfully, we may make use of computer simulations to aid our calculations.

\subsection{Event simulation}

When colliding composite particles such as protons, the calculation of the cross section in Equation (5.1) is not a simple matter of collecting the relevant diagrams and adding them together. The interaction of the two protons, as mentioned above, will be interactions between the constituents of the proton. The amount of each type of parton in the proton is given by a parton density function (PDF), $f_{i}(x)$, where $x$ is the momentum fraction carried by parton $i$. In a proton, $i$ can be any one of the six quarks or a gluon. In addition to the usual phase-space integration, we must convolve the parton-level cross sections with their PDFs. The cross section for a given final state, $F$, initiated by the collision of two protons, $I$ and $J$, is given by $1 \mathbf{1 3 6}$

$$
\sigma(I J \rightarrow F X)=\sum_{i \in I, j \in J} \int d x_{1} d x_{2} f_{i}\left(x_{1}\right) f_{j}\left(x_{2}\right) \hat{\sigma}(i j \rightarrow F)
$$


where $X$ is the collection of particles resulting from the collision which are not involved in our final state of interest, and $\hat{\sigma}$ is the parton-level cross section. Performing this integration cannot, in general, be done analytically due to the presence of the PDFs.

In that case, we use Monte Carlo techniques to evaluate the integral. Given an integral, $\mathcal{I}=\int_{a}^{b} f(x) d x$, we can approximate it by taking $N$ uniformly distributed random values $x_{i} \in[a, b]$, such that

$$
\mathcal{I} \approx \frac{1}{N} \sum_{i=1}^{N}(b-a) f\left(x_{i}\right)=\frac{1}{N} \sum_{i=1}^{N} W_{i}
$$

where $W_{i}$ is called the weight, and the integral is simply the average of the weights.

The process of simulation involves calculating the weight at a set of uniformly chosen random points in the PDF and phase space directions. These weights are then normalized to the maximum weight, $R_{i}=W_{i} / W_{\mathrm{MAx}}$, and those with $R_{i}>R$ for some $R \in(0,1)$ are selected, until the desired number of events is accrued. This process is known as unweighting.

An event may be factorized into two main subprocesses: the hard process at the parton-level, and the shower, decay and hadronization. Each of these has its own set of specialized computer tools. The most common toolchain for simulating events at the LHC is FeynRules [106], MadGraph5_aMC@NLO [137, 108], Pythia [138], and Delphes [139]. The first of these, FeynRules, translates a Lagrangian into model files that allow MadGraph5_aMC@NLO to construct matrix elements and calculate partonlevel events which are then passed to Pythia for showering and hadronization, and finally Delphes simulates the effects of the detector components. The output of this is a cross section, or using Equation (5.1), a number of events. This number of events may then be compared to an observed number of events coming from a given search, where some form of kinematic cuts (requirements on, e.g., $p_{T}$ or $\mathbb{E}_{T}$ for the 

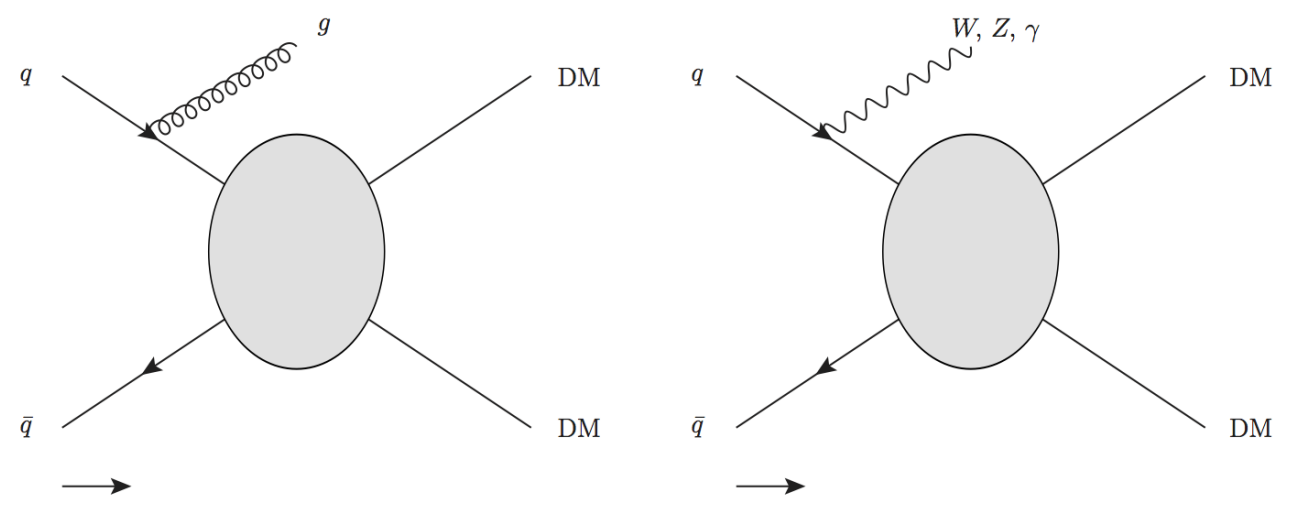

Figure 5.2. Possible visible missing transverse momentum interactions. An initial-state gluon or gauge boson would provide a visible signal against which to balance the $\mathbb{E}_{T}$ from the DM particles.

specific search) and triggers (thresholds on kinematic variables required by detector components to record the event) have been imposed. We are now in a position to discuss constraints on our models from searches for NP at the LHC.

\subsection{Direct search constraints from LHC data}

Against what sort of searches should we compare our models? If our model had only a single new particle - the DM candidate - as in singlet extensions of the SM [98], our best chance to find NP would be through the use of "mono-X" searches (where $\mathrm{X}$ could be a jet, a $W, Z$, or $h$ boson, or a photon), as in Figure 5.2.

Without the initial (ISR) or final (FSR) state radiation, there would be no visible signal against which to balance the $\mathbb{E}_{T}$, meaning we would see nothing in the detector. The mono-X searches look for ISR/FSR signals with large $\mathbb{E}_{T}$ and provide limits on the spin-independent and -dependent cross section which are better than those of direct detection in the low-DM-mass region $\left(m_{\mathrm{DM}} \lesssim 10 \mathrm{GeV}\right)$ [140, 141, 82, 81]. The analyses in these searches use an effective field theory approach to model the DM-SM interactions. 


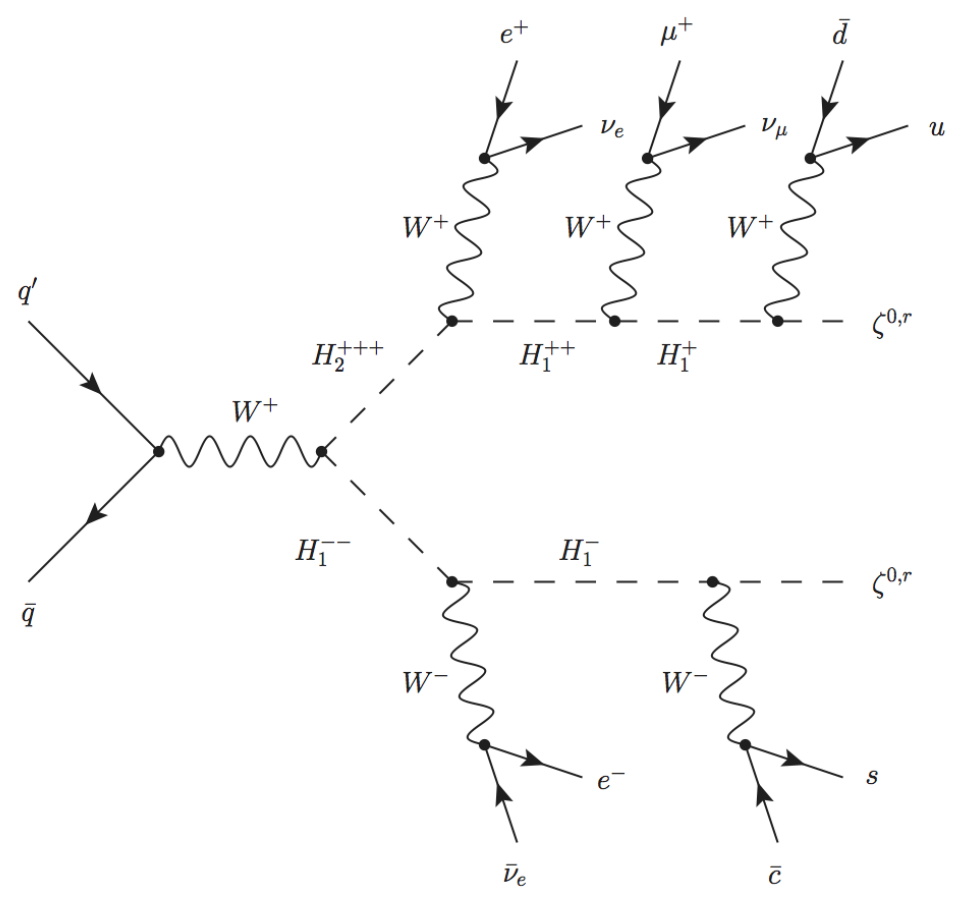

Figure 5.3. Sample Feynman diagram for a collider process in the large multiplet models. The final state would consist of four jets, an electron, positron, anti-muon, and $\mathbb{E}_{T}$ from the neutrinos as well as the two $\zeta^{0, r}$. The generally small mass splitting between the $H_{1}^{ \pm 1}$ and the $\zeta^{0, r}$ means that the jets would be very soft.

For models that add much more than a single particle, such as our large multiplet models, we can produce many more states than just the DM candidate. The heavier states will then decay to a lighter state from $\Sigma$ and a $W$ or $Z$ boson, which will in turn decay to SM particles. This will continue until we are left with two $\zeta^{0, r}$ particles and a large number of SM particles, as in Figure 5.3.

Currently, searches for jets $+\mathbb{E}_{T}$ are typically only constraining for signals produced via QCD-strength interactions because of the large $W / Z+$ jets backgrounds; nevertheless, we include them here for completeness. Signatures involving leptons $+\mathbb{E}_{T}$, for which the SM backgrounds are smaller, are typically more constraining for models 
in which the new physics is produced with electroweak-strength cross sections, as in our models. These leptons $+\mathbb{E}_{T}$ signatures will be responsible for the direct-search constraints on our models. We take advantage of the large number of possible pairs of scalars that can be produced via s-channel $\gamma, Z$, or $W$ exchange in our models by simulating all such processes and combining the resulting signal events. We compare to searches performed by the ATLAS and CMS collaborations using data from the LHC at a centre-of-mass energy of $8 \mathrm{TeV}$ (LHC8), collected in 2012.

The following searches were those which had similar final states to our own models:

- ATLAS opposite-sign dileptons with $\mathbb{E}_{T}$ and no jets [142];

- ATLAS trilepton plus $\mathbb{E}_{T}[\mathbf{1 4 3}, 144$ (the two studies employ distinct methodologies, and are compared separately);

- ATLAS four or more leptons [145];

- ATLAS dileptons with razor variables [146];

- ATLAS hadronic di- $\tau$ plus $\mathbb{E}_{T}$ [147];

- ATLAS same-sign dileptons plus jets [148];

- ATLAS monojet [149, 150];

- ATLAS multi-jets plus $\mathbb{E}_{T}$ [151];

- CMS multi-leptons (dilepton, trilepton, multi-lepton) with $\mathbb{E}_{T}$ [152]; and - CMS monojet [153].

We performed the simulation following the methodology of Ref. [154]. For each of the four mass slices, $m_{\zeta^{0, r}}=80,120,180$, and $300 \mathrm{GeV}$ in each model, we generated parameter points on a grid in $R_{1}$ and $R_{2}$ (the green points shown in Figure 5.4), focusing on the region that is allowed by the theoretical and indirect experimental constraints from Sections 4.2, 4.3 and 5.4 (below the cyan curves in Figure 5.4). 


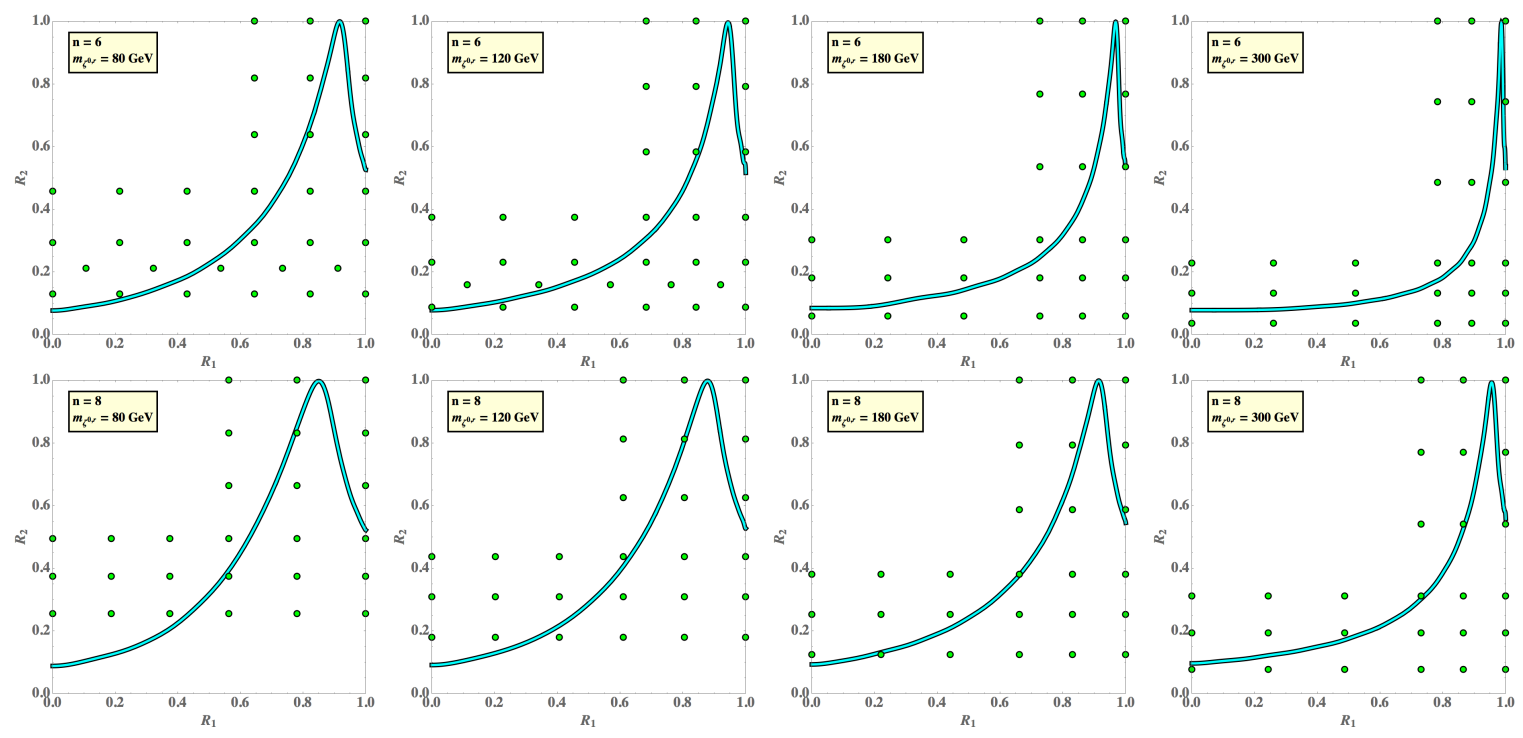

FiguRE 5.4. Scan points for the LHC direct searches. The region below the cyan curve is allowed by the theoretical and indirect constraints derived in Sections 4.2, 4.3 and 5.4 .

We also simulated a few additional points for added sensitivity in regions where the experimental sensitivity was varying rapidly.

We do not simulate parameter points for which $\Delta m_{0} \equiv m_{\zeta^{0, i}}-m_{\zeta^{0, r}}<10 \mathrm{GeV}$. As we will see, this compressed-spectrum region is particularly difficult to detect because the leptons resulting from the decays tend to be too soft to pass the selection cuts. Indeed, we will find that parameter regions with $\Delta m_{0} \lesssim 20 \mathrm{GeV}$ cannot be excluded using the LHC8 searches that we recast. This parameter region could be picked up by jets $+\mathbb{E}_{T}$ searches, but we find that the scalar pair production cross sections are too small for LHC8 to have any sensitivity in this channel. Very small mass splittings could result in macroscopic decay lengths for charged scalars; while dedicated searches for such signatures exist, we will not consider them here.

We used an inclusive production methodology due to the large particle content of the model and the complicated interplay between production and decay rates, and 
the identification and acceptance rates at the LHC. Using MadGraph5_aMCONLO, we generated 50,000 events for each of the possible pairs of scalars that can be produced via $s$-channel $W^{ \pm}, \gamma, Z$, or $h$ exchange, in association with zero or one hard jet(s). The new scalars were produced in pairs due to the $Z_{2}$ symmetry - under this symmetry, $\Sigma$ is odd and the SM particles are all even, so to conserve this "DM parity," we require two multiplet members in each interaction. This amounted to 32 possible scalar pairings in the $n=6$ model and 44 in the $n=8$ model.

The addition of a hard jet to our production step introduces a potential problem for the analysis: the event with one hard jet at the matrix-element level may be indistinguishable from a matrix-element level event with no hard jet, but which produces one during the parton shower. The possible double-counting cross section contributions leads to the concept of matching and merging [155, 156, 138]. The parton-level events are calculated using the matrix element technique (e.g., by MadGraph), then the shower is performed (e.g., by Pythia). The events are matched at some scale for the jet $p_{T}$, and how well these samples are matched can be determined by looking at how smooth is the distribution in the matching scale [157].

We do not consider gluon fusion production of $h$ in our main analysis, despite it being the main production mechanism at the LHC. Over much of the parameter space, the coupling of pairs of scalars to the Higgs is much smaller than the corresponding coupling to $Z$ or photons. Since the cross section most affected by the inclusion of this production mode is many orders of magnitude smaller than the production mode that causes the exclusion, leaving out $g g \rightarrow h$ will have no effect on the final result. As a check, we investigated the effects of including the effective $g g h$ vertex by adding the HiggsEffective model file $\mathrm{2}^{2}$ to our FeynRules model. We found that the addition 2 http://feynrules.irmp.ucl.ac.be/wiki/HiggsEffectiveTheory 
of this production mechanism does not affect the overall exclusion, adding far fewer than a single event to any of the parameter scan points. We are then justified in omitting gluon fusion Higgs production to reduce the computation time required.

Each simulated event sample was then matched and merged, decayed, showered, and hadronized using Pythia 6 [138. The events were then passed through the Delphes 3 [139] detector simulation multiple times: for each of the experimental searches that we recast, we adjusted the identification efficiencies and jet algorithm settings in Delphes 3 to match the working point used in the experimental analysis. We then used the Seer [158 analysis program to apply the trigger and kinematic cuts of each experimental analysis and determine the total signal cross section from all production processes that fall into each of the signal regions. Additional Gaussian smearing of the $\mathbb{E}_{T}$ beyond that already present in Delphes 3 was introduced via Seer to account for the effect of pileup [159]. This has been shown to be necessary in order to reproduce the event numbers in cut-flow tables for each of the experimental searches [154].

Our models include parameter regions in which the spectrum is highly compressed. In such regions, simulation problems can arise because the automatic parton-level computation of partial widths and branching ratios using MadWidth 108 does not take into account hadronization effects arising from decays involving a highly off-shell $W$ or $Z$ boson.

For example, consider the case of $H_{1}^{ \pm} \rightarrow \zeta^{0, r}+W^{ \pm}$decay for a parameter point at which the mass splitting $m_{H_{1}^{+}}-m_{\zeta^{0, r}}$ is larger than the mass of a $\pi^{ \pm}$but smaller than the mass of the $\pi^{ \pm} \pi^{0}$ system, as in Table 5.1. Physically, a hadronically-decaying offshell $W$ will emerge in this case as a single $\pi^{ \pm}$. However, MadWidth treats all decay products as bare partons and will decay the off-shell $W$ to a quark-antiquark pair. 


\begin{tabular}{ccc}
\hline \hline State & Mass $[\mathrm{GeV}]$ & $\Delta m_{i, i-1}[\mathrm{GeV}]$ \\
\hline$\zeta^{0, r}$ & 80.0000 & - \\
$H_{1}^{ \pm}$ & 80.1960 & 0.196 \\
$H_{1}^{ \pm \pm}$ & 80.8320 & 0.636 \\
$\zeta^{ \pm 3}$ & 82.1524 & 1.3204 \\
$H_{2}^{ \pm \pm}$ & 89.2535 & 7.1011 \\
$H_{1}^{ \pm}$ & 89.8254 & 0.5719 \\
$\zeta^{0, i}$ & 90.0000 & 0.1746 \\
\hline \hline
\end{tabular}

TABLE $\overline{\overline{5.1 .} \text { Mass spectrum and splittings for a sample point }}$ in the compressed-spectrum region, $\Delta m_{0}=10 \mathrm{GeV}$. For this point, $n=6$, $m_{\zeta^{0, r}}=80 \mathrm{GeV}, R_{1}=0.215$ and $R_{2}=0.130$. Compare the charged pion mass at $0.140 \mathrm{GeV}$.

When Pythia [138] hadronizes the resulting quark-antiquark pair, it tries to create two pions, finds that this is forbidden by the available phase space, and discards the event.

This is a problem because it removes generated events that could otherwise potentially be detected via, e.g., a hard initial-state jet radiation. We solve this problem by manually removing any hadronic $W$ branching ratio from the MadGraph param_card files in which the $W$ invariant mass is less than the mass of two correspondinglyflavored mesons and rescaling the remaining $W$ decay branching ratios to sum to one.

Should we worry about changing the potential detector signature of the very soft $W$ boson? A simple kinematical argument indicates no. In the rest frame of the decaying scalar, the momentum of the outgoing decay products must satisfy, e.g., $\left|p_{\zeta^{0, r}}\right|+\left|p_{\text {jets }}\right| \leq m_{H_{1}^{+}}-m_{\zeta^{0, r}}$ for the above example. In this frame the decay product(s) of the $W$ will have momentum less than the scalar mass splitting. To make the $W$ 
decay product(s) visible in the detector, they must be boosted to an ultrarelativistic velocity; for example, a $10 \mathrm{GeV}$ charged pion requires a relativistic boost factor $\gamma \simeq 70$. This requires that the parent scalar itself be boosted by the same relativistic boost factor: for a scalar mass around $80 \mathrm{GeV}$, this implies a transverse momentum of more than $5 \mathrm{TeV}$, which is not even kinematically possible at the $8 \mathrm{TeV}$ LHC.

At each simulated parameter point, we select as most sensitive the experimental search that yields the largest value of $\log _{10}\left(N_{\text {sig }} / N_{95}\right)$, where $N_{\text {sig }}$ is the number of signal events computed from the cross section in the signal region for the appropriate integrated luminosity and $N_{95}$ is the corresponding $95 \%$ confidence level exclusion threshold from the experimental analysis. If $\log _{10}\left(N_{\mathrm{sig}} / N_{95}\right)>0$ for the most sensitive search, the parameter point is excluded since that would mean that there were more signal events than the limit set by the experimental analyses. We do not combine significances from different search channels - to do so would require knowledge of the statistical correlations among the various searches which we do not have. In between parameter points, we interpolate linearly in the value of $\log _{10}\left(N_{\mathrm{sig}} / N_{95}\right)$ found for the most sensitive search at each point.

We find that the most sensitive analyses are the ATLAS dilepton, trilepton and four-lepton searches. Several other searches also exclude parameter space for low values of $m_{\zeta^{0, r}}$, but this is primarily due to the large pair production rates when the scalar masses are low, which overcome the inherently smaller acceptances and/or larger backgrounds in these searches.

We present our results in Figure 5.5 for the $n=6$ model and Figure 5.6 for the $n=8$ model. These plots are in the $R_{1}-R_{2}$ plane as described in Section 3.2 which allows us to explore the parameter space in better detail based on other constraints. 
Each plot consists of three lines and twenty-seven points overlaid on a coloured region. The darker the colour of the region around a point, the higher the value of $\log _{10}\left(N_{\text {sig }} / N_{95}\right)$. The green points correspond to the parameter points chosen for the scans, as in Figure 5.4. The solid black line encompasses the region excluded at 95\% confidence level by one or more of the searches used. The dashed black line indicates how the excluded region would be expanded if the signal cross section were increased by a factor of 1.2 . This $k$-factor is used to account for the difference between leadingorder cross sections (as calculated in this analysis) and higher-order cross sections which were not included. The thick horizontal pink line indicates the $R_{2}$ value that corresponds to $\Delta m_{0} \equiv m_{\zeta^{0, i}}-m_{\zeta^{0, r}} \lesssim 20 \mathrm{GeV}$. Below this line, the overall spectrum of the models is very highly compressed and a separate detailed analysis would be required. The region above the thick teal curve is excluded by the unitarity Section 4.3 and alternate minima Section 4.2 constraints from the previous chapter, along with the $S T U$ and $h \rightarrow \gamma \gamma$ constraints which will be discussed later in this chapter. This curve illustrates why we did not generate any scan points in the upperleft of each scan region. We now give the interpretation of the excluded regions in each mass slice for both of our models.

\subsubsection{Results for the $n=6$ model.}

In the $n=6$ model, the parameter space is strongly excluded at low $m_{\zeta^{0, r}}$ so long as $\Delta m_{0}>20 \mathrm{GeV}$. As $m_{\zeta^{0, r}}$ increases, the excluded region shrinks until only a small sliver of parameter space around $R_{2} \sim 0.3$ is excluded for $m_{\zeta^{0, r}}=180 \mathrm{GeV}$. LHC8 makes no exclusion at $m_{\zeta^{0, r}}=300 \mathrm{GeV}$ or above.

For $m_{\zeta^{0, r}}=80 \mathrm{GeV}$, shown in the upper left panel of Figure 5.5, the total scalar pair production cross section before cuts ranges from $\sim 30 \mathrm{pb}$ for small $R_{2}$ to $\sim$ $16 \mathrm{pb}$ for large $R_{2}$. The ATLAS trilepton $+\mathbb{E}_{T}$ searches [143, 144] provide the 
most sensitive exclusion for all parameter points scanned. Due to the large signal cross sections, even small acceptance rates result in large numbers of events in the experimental signal regions, resulting in the strong exclusion as indicated by dark colours. The excluded region ends abruptly at $\Delta m_{0} \simeq 20 \mathrm{GeV}$. For $R_{2}$ values below this boundary, the highest lepton $p_{T}$ is rarely above $10 \mathrm{GeV}$, unless the scalars are produced boosted or in association with an energetic jet, which is rare. The ATLAS and CMS offline leptonic triggers are typically set at $p_{T} \geq 10 \mathrm{GeV}$, resulting in a drastic plunge in acceptance for softer leptons. Similarly, the $\mathbb{E}_{T}$ tends to be low when the spectrum is compressed; most (but not all) of the searches require $\mathbb{E}_{T} \geq 50 \mathrm{GeV}$, further reducing sensitivity in the compressed-spectrum region. This kinematic acceptance boundary is apparent in many of the exclusion plots for both the $n=6$ and $n=8$ models.

For $m_{\zeta^{0, r}}=120 \mathrm{GeV}$, shown in the upper right panel of Figure 5.5, the total scalar pair production cross section before cuts ranges from $\sim 8.2 \mathrm{pb}$ for small $R_{2}$ to $\sim 3.5 \mathrm{pb}$ for large $R_{2}$. The ATLAS trilepton $+\mathbb{E}_{T}$ searches [143, 144] provide the most sensitive exclusion for $R_{2} \lesssim 0.5$, in particular the search region with same-flavor opposite-sign dilepton mass smaller than the $Z$ mass, which picks up the off-shell $Z$ boson decays. For $R_{2} \gtrsim 0.5$ and $R_{1} \gtrsim 0.75$, the most sensitive search is the ATLAS four-lepton search [145], due to the smaller backgrounds and the predominance of $\zeta^{0, i} \rightarrow \zeta^{0, r} Z$ and $H_{2}^{+} \rightarrow H_{1}^{+} Z$ decays in this region (see Figures 3.5 and 3.6). The unexcluded region in the upper right corner of this plot results from the loss of the four-lepton signatures as the decays involving $W$ emission go on shell (the resulting two-lepton final states suffer from larger backgrounds).

For $m_{\zeta^{0, r}}=180 \mathrm{GeV}$, shown in the lower left panel of Figure 5.5, the total scalar pair production cross section before cuts ranges from $\sim 1.7 \mathrm{pb}$ for small $R_{2}$ 
to $\sim 0.7 \mathrm{pb}$ for large $R_{2}$. The ATLAS triplepton $+\mathbb{E}_{T}$ searches [143, 144] are responsible for the exclusion between $R_{2}$ values of about 0.2 and 0.3 , while the ATLAS four-lepton search [145] is responsible for the thin strip of excluded parameter space near $R_{1} \simeq 0.75$. Within the parameter region allowed by theoretical and indirect experimental constraints, only a small sliver of parameter space around $R_{2} \sim 0.3$ is excluded by the ATLAS triplepton $+\mathbb{E}_{T}$ searches. The largest contribution to the trilepton $+\mathbb{E}_{T}$ signal is from the $H_{2}^{ \pm \pm} H_{2}^{\mp}$ pair production mode, which decays in this region of parameter space mainly to $H_{1}^{ \pm} W^{ \pm} H_{1}^{\mp} Z$, followed by very soft decays of the $H_{1}^{ \pm}$states. As $R_{2}$ increases from 0.3 to 0.4 , the $H_{2}^{\mp}$ decay transitions to $\zeta^{0, r} W^{\mp}$ due to the nearby kinematic threshold (see Figure 3.6) so that the third lepton is lost. The production cross sections for the heavier scalars also decrease with increasing $R_{2}$.

For $m_{\zeta^{0, r}}=300 \mathrm{GeV}$, shown in the lower right panel of Figure 5.5, the total scalar pair production cross section before cuts ranges from $\sim 0.2 \mathrm{pb}$ for small $R_{2}$ to $\sim 0.08 \mathrm{pb}$ for large $R_{2}$. After accounting for branching ratios and acceptances, it is clear that there is simply insufficient luminosity collected at LHC8 to result in any sensitivity to the model for this and higher masses.

\subsubsection{Results for the $n=8$ model.}

In the $n=8$ model, the parameter space at low $m_{\zeta^{0, r}}$ is again excluded so long as $\Delta m_{0} \gtrsim 20 \mathrm{GeV}$. The excluded region shrinks rapidly with increasing $m_{\zeta^{0, r}}$ until LHC8 can make no exclusion at $m_{\zeta^{0, r}}=180 \mathrm{GeV}$ or above.

The most important difference between the two models is that the maximum mass splitting between the lightest and heaviest scalars, $\Delta m_{0}^{\mathrm{MAX}} \equiv m_{\zeta^{0, i}}-m_{\zeta^{0, r}}$, is considerably smaller in the $n=8$ model than in the $n=6$ model, by about a factor of two (see Table 3.1). Therefore, a much larger fraction of the allowed $n=8$ model parameter space lies in the compressed region with $\Delta m_{0}<20 \mathrm{GeV}$ (below the thick 
light pink horizontal line in Figure 5.6). The smaller mass splittings over the entire $n=8$ parameter space result in softer leptons, jets, and $\mathbb{E}_{T}$ and hence overall lower acceptance rates in the LHC8 analyses. This results in weaker exclusions in the $n=8$ model in spite of the larger number of scalars, their higher weak and electric charges (leading to more like-sign leptons from longer decay chains), and the correspondingly higher total scalar pair production cross sections before cuts. The emitted leptons are just too soft.

For $m_{\zeta^{0, r}}=80 \mathrm{GeV}$, shown in the upper left panel of Figure 5.6, the total scalar pair production cross section before cuts ranges from $\sim 90 \mathrm{pb}$ for small $R_{2}$ to $\sim 62 \mathrm{pb}$ for large $R_{2}$. The entire parameter space with $R_{2} \gtrsim 0.5$ is excluded. Similarly to the $n=6$ model, the excluded region ends around $\Delta m_{0} \simeq 20 \mathrm{GeV}$, below which the highest lepton $p_{T}$ is rarely above $10 \mathrm{GeV}$, so that most events fail the offline leptonic triggers. The most sensitive exclusion comes from the published ATLAS trilepton $+\mathbb{E}_{T}$ study [144]. This study captures a broader range of signal characteristics than the corresponding conference note [143], and includes signal regions that are more sensitive to low dilepton invariant masses (called the $S R 0 \tau a$ bins $1-4$ in Ref. [144]). In particular, the best constraints on the $n=8$ model come from $S R 0 \tau a$ bin 2 , in which the invariant mass of the same-flavor opposite-sign lepton pair lies between 12 and $40 \mathrm{GeV}$, the transverse mass of the remaining lepton and the $\mathbb{E}_{T}$ lies below $80 \mathrm{GeV}$, and $\mathbb{E}_{T}>90 \mathrm{GeV}$. The most constraining bin in Ref. [143] is similar, but with a softer cut on $\mathbb{E}_{T}$, which results in larger background. The ATLAS four-lepton search [145] also redundantly excludes part of the parameter space, but loses sensitivity for larger values of $R_{1}$, where the dominant decay of $H_{2}^{ \pm \pm}$changes from $H_{1}^{ \pm \pm} Z$ to $H_{1}^{ \pm} W^{ \pm}$. 
For $m_{\zeta^{0, r}}=120 \mathrm{GeV}$, shown in the upper right panel of Figure 5.6, the total scalar pair production cross section before cuts ranges from $\sim 20$ pb for small $R_{2}$ to $\sim 12 \mathrm{pb}$ for large $R_{2}$. The excluded region is understood similarly to the previous mass slice, with the same mechanisms at work. When the smaller cross sections are combined with branching ratios and acceptance rates, the signal events in the published ATLAS trilepton $+\mathbb{E}_{T}$ selection [144] wind up falling into multiple signal bins, rather than being as concentrated in a single bin as in the $m_{\zeta^{0, r}}=80 \mathrm{GeV}$ slice. Since we do not have information on the correlations of the systematic uncertainties among different bins, we cannot employ the full usefulness of the $C L_{s}$ method to combine the bins to extend the exclusion boundaries. In addition, decays to pairs of off-shell $Z$ bosons are common, which reduces the trilepton signal from $W Z$ production. A four-lepton search is intrinsically less sensitive than a trilepton search due to the smaller leptonic branching ratio of the $Z$ compared to the $W$.

For $m_{\zeta^{0, r}}=180 \mathrm{GeV}$, shown in the lower left panel of Figure 5.6, the total scalar pair production cross section before cuts ranges from $\sim 4.7 \mathrm{pb}$ for small $R_{2}$ to $\sim 2.7 \mathrm{pb}$ for large $R_{2}$. None of the scanned parameter space is excluded by LHC8 searches. A small region of parameter space at $R_{2} \gtrsim 0.9$ and $R_{1} \sim 0.7$ would be excluded if a signal $k$-factor of 1.2 were included. The sensitivity in this region comes from the ATLAS opposite-sign dileptons $+\mathbb{E}_{T}$ search with no jets [142], which is sensitive to the off-shell $Z$ decays from the $H_{2}^{ \pm \pm}$and $\zeta^{0, i}$ that are produced in association with $H_{1}$ states. Jets from the decays of the $H_{1}$ states are soft enough that they evade the jet veto in the search.

For $m_{\zeta^{0, r}}=300 \mathrm{GeV}$, shown in the lower right panel of Figure 5.6, the signal rate after cuts in any channel is less than $10 \%$ of that required for an exclusion over the entire scanned parameter space. 

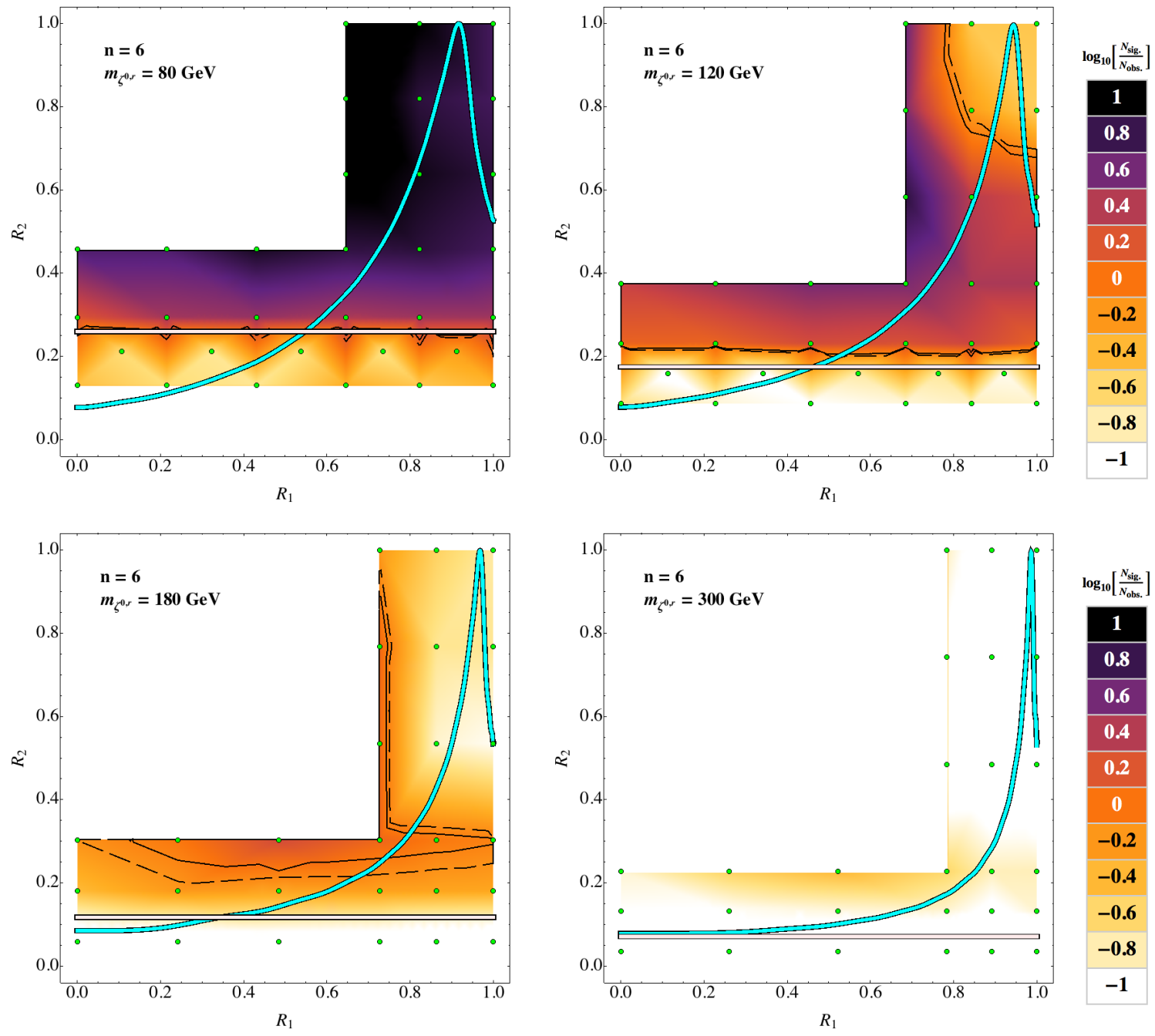

Figure 5.5. Combined exclusion regions in the $n=6$ model from all examined LHC8 analyses. The darker-colored regions above and to the left of the solid black line are excluded at $95 \%$ confidence level by at least one analysis. The dashed black line indicates how the excluded region would expand if the signal cross sections were all increased by a $k$-factor of 1.2 . The parameter region below the thick teal curve is allowed by the theoretical and indirect experimental constraints. The thick horizontal light pink line indicates the $R_{2}$ value at which $\Delta m_{0} \equiv$ $m_{\zeta^{0, i}}-m_{\zeta^{0, r}}=20 \mathrm{GeV}$. 

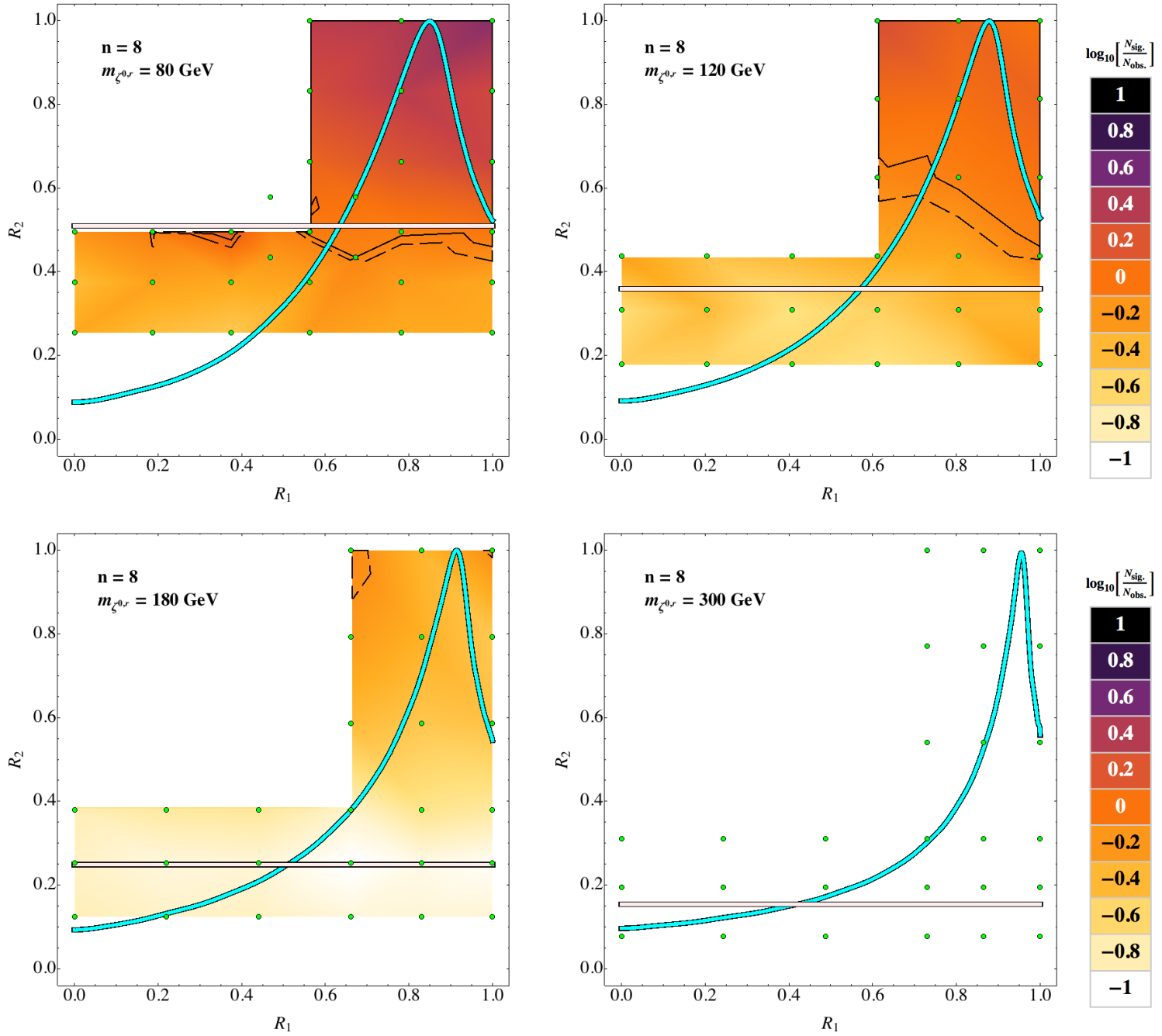

Figure 5.6. Combined exclusion regions in the $n=8$ model from all examined LHC8 analyses. The darker-colored regions above the solid black line are excluded at 95\% confidence level by at least one analysis. The dashed black line indicates how the excluded region would expand if the signal cross sections were all increased by a $k$-factor of 1.2 . The parameter region below the thick teal curve is allowed by the theoretical and indirect experimental constraints. The thick horizontal light pink line indicates the $R_{2}$ value at which $\Delta m_{0} \equiv m_{\zeta^{0, i}}-m_{\zeta^{0, r}}=20 \mathrm{GeV}$. 


\subsubsection{Summary of direct search constraints.}

In the above, we probed our large multiplet models by recasting a subset of the searches for supersymmetric particles performed by the CMS and ATLAS collaborations using LHC8 data. We have seen that these searches provide nontrivial constraints on the parameter space. The strongest of these constraints is on the low-mass regions $\left(m_{\zeta^{0, r}}=80,120 \mathrm{GeV}\right)$, specifically where the mass splitting is $m_{\zeta^{0, r}} \gtrsim 20 \mathrm{GeV}$. The loss of sensitivity in the regions where $m_{\zeta^{0, r}} \lesssim 20 \mathrm{GeV}$ is due to the softness of the decay products, meaning that they fail to trigger the detector. The LHC8 constraints rapidly disappear for $m_{\zeta^{0, r}}>120 \mathrm{GeV}$, until there is little sensitivity above $m_{\zeta^{0, r}}=180 \mathrm{GeV}$. These results are in general agreement with the LHC8 constraints on the electroweak gauginos in supersymmetric models studied in Ref. [154], in which the same collection of LHC8 searches showed sensitivity to masses of the lightest supersymmetric particle primarily smaller than $100 \mathrm{GeV}$.

The LHC8 constraints on our models are much weaker than those on the scalar septet extension of the SM Higgs sector [160 studied in Ref. [161]. Using similar searches from ATLAS and CMS, Ref. [161] found that septet masses below $\sim 400 \mathrm{GeV}$ were robustly excluded, and that multi-lepton searches provided the strongest constraints. The dramatically enhanced LHC8 sensitivity to the septet model compared to our sextet and octet models is due to the nonzero vacuum expectation value carried by the septet and the mixing between the neutral scalar in the septet model and the SM Higgs boson in that model. These features allow the lightest septet state to decay into SM particles, particularly into energetic gauge boson pairs, leading to final states with two to four high-energy leptons. The mass reach of the LHC8 exclusion is then controlled by the decrease in the production cross section with increasing scalar masses. 
With Run 2 of the LHC underway at $13 \mathrm{TeV}$ centre-of-mass energy, the next round of searches will likely provide stronger constraints on our models, extending to higher scalar masses. The pair production cross section for fixed scalar masses grows with increasing proton-proton centre-of-mass energy due mainly to the rise in the antiquark parton density. Higher integrated luminosity will also aid the searches, but with diminishing returns. The analyses that we use compare an observed event number to theoretical predictions for the background cross section; unless these backgrounds can be normalized from data, such searches suffer from systematic uncertainties in the background prediction that ultimately limit the benefit of greater luminosity. In addition, higher instantaneous luminosity will necessitate an increase in trigger thresholds,

which will further reduce the sensitivity to the compressed spectrum regions. Discovery prospects at a higher energy proton-proton collider would face similar issues.

Moving from these tree-level, direct search constraints, we next examine how our large multiplet models will affect SM processes at the loop level, and derive constraints from measurements of these processes.

\subsection{Effects of new physics in loops affecting SM processes}

To detect NP such as DM, it must couple to the SM in some way, otherwise we would be unable to probe its nature beyond its gravitational effects. Tree-level processes involving NP are constrained by direct searches, as seen in the previous section. On the other hand, if the NP does couple to the SM as we expect it must, then it will affect SM processes at the loop level. As measurements and calculations of SM processes improve, discrepancies between these two may indicate the presence of NP. However, as we pointed out at the beginning of this section, the agreement between SM calculations and experimental results leaves few places for NP to hide. 


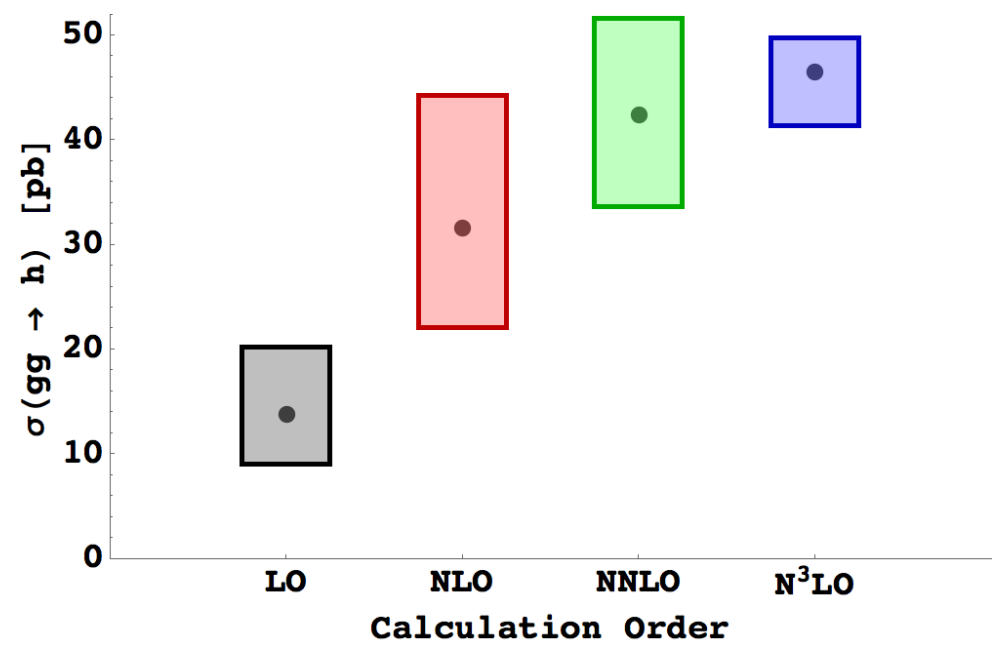

Figure 5.7. Comparison between QCD loop-order calculations of gluon fusion Higgs production. The number of "N"s indicate one less than the number of loops ( $g g \rightarrow h$ at leading-order is 1-loop). Plot uses values from Table 3 of Ref. 162. The height of each box corresponds to the estimated uncertainty from the missing higher orders of the calculation.

SM processes will also be affected by SM particles at the loop level. For example, in Figure 5.7, the calculation of the inclusive Higgs production from gluon fusion is shown at the one-loop (LO), two-, three-, and four-loop level (NLO, NNLO, and $\mathrm{N}^{3} \mathrm{LO}$ in QCD, respectively [162]). The increased precision of the calculation allows better comparison to the measured production cross section. Notice that, although the LO and NLO calculations differ by a large amount, subsequent orders in the calculation shows complete agreement.

The members of $\Sigma$ couple to the Higgs boson, as well as to the photons and the $W$ and $Z$ bosons. The decay of the Higgs to two photons, $h \rightarrow \gamma \gamma$, has been observed, and an upper-limit has been placed on the decay to a $Z$ boson and a photon, $h \rightarrow Z \gamma$, both at the LHC. Since our multiplet couples to the particles on either side of the loop (the $h, Z$, and $\gamma$ ), it can run in the loops and will affect the decay rates. In 
the following sections, we will see how these measurements lead to constraints on the parameter space of our large multiplet models.

\subsubsection{STU.}

Thanks to experiments such as MuLan, Tevatron, LEP, and LHC, we have very precisely measured certain parameters of the electroweak sector of the SM. We can relate some of these to compare experimental measurements. NP contributions to any of these parameters may then be indicated by discrepancies among the measurements. For example, three of the most precisely measured parameters are the electromagnetic fine structure constant, $\alpha_{\mathrm{EM}}$, the Fermi constant, $G_{F}$, and the mass of the $Z$ boson, $m_{Z}$ [6]. We can use as a standard of reference the value of the weak mixing angle that employs these variables,

$$
\sin ^{2} 2 \theta_{W}=\frac{4 \pi \alpha_{\mathrm{EM}}}{\sqrt{2} G_{F} m_{Z}}
$$

The deviation from the SM prediction due to new physics is then [163, 164

$$
s_{W}^{2}=\hat{s}_{W}^{2}\left[1-\frac{\hat{c}_{W}^{2}}{\hat{c}_{W}^{2}-\hat{s}_{W}^{2}}\left(\delta \hat{\Pi}_{\gamma \gamma}(0)-\delta \hat{\Pi}_{Z Z}\left(m_{Z}^{2}\right)+\frac{\delta \Pi_{W W}(0)}{m_{W}^{2}}\right)\right],
$$

where $s_{W}^{2}=\sin ^{2} \theta_{W}$ is the corrected value, $\hat{s}_{W}^{2}=1-\hat{c}_{W}^{2}$ is the tree-level SM value, $\delta \Pi_{a b}(x)$ is the one-loop contribution to the two-point $a-b$ function, and $\delta \hat{\Pi}(x) \equiv$ $\delta \Pi_{a b}(x) / x$. We can then directly calculate what effect the NP will have on the predicted value of $s_{W}^{2}$. In the SM, the contributions will vanish so that $s_{W}^{2}=\hat{s}_{W}^{2}$.

If we consider the scattering of two light fermions, as in Figure 5.8, we will have a contribution from the two gauge propagators and the self-energy of the gauge particle itself,

$$
\left(A g_{\alpha \mu}+B q_{\alpha} q_{\mu}\right)\left[\Pi\left(q^{2}\right) g^{\mu \nu}+\Delta\left(q^{2}\right) q^{\mu} q^{\nu}\right]\left(A g_{\nu \beta}+B q_{\nu} q_{\beta}\right)
$$




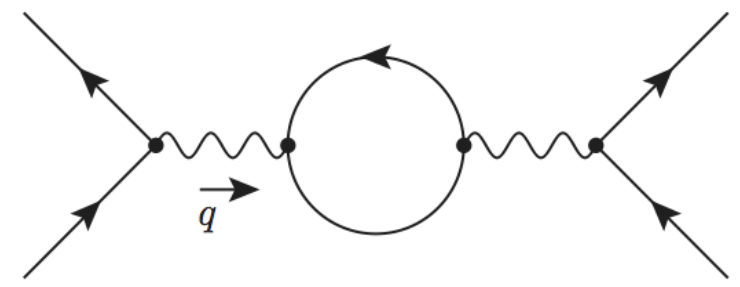

FiguRE 5.8. Feynman diagram of the scattering of light fermions showing the one-loop fermion correction to the gauge boson propagator.

The propagator momenta, $q^{2}$, will all couple with the gamma matrices of the external fermions and result in terms proportional to the fermion masses. At high energy, these masses will be negligible and so, in the above expression, we can ignore terms containing $q^{\lambda}$. The part of interest is then the two-point function $\Pi\left(q^{2}\right)$.

In the limit $q^{2} \rightarrow 0$, we consider the expansion of this function,

$$
\Pi\left(q^{2}\right) \approx \Pi(0)+q^{2} \Pi^{\prime}(0)+\cdots
$$

where $\Pi^{\prime}(0)=\partial \Pi /\left.\partial q^{2}\right|_{q^{2}=0}$.

We will be interested in the following self-energies: $W \rightarrow W, Z \rightarrow Z, Z \leftrightarrow \gamma$, and $\gamma \rightarrow \gamma$. The are given by

$$
\begin{aligned}
\Pi_{W W}\left(q^{2}\right) & =\Pi_{W W}(0)+q^{2} \Pi_{W W}^{\prime}(0) \\
\Pi_{Z Z}\left(q^{2}\right) & =\Pi_{Z Z}(0)+q^{2} \Pi_{Z Z}^{\prime}(0) \\
\Pi_{Z \gamma}\left(q^{2}\right) & =q^{2} \Pi_{Z \gamma}^{\prime}(0) \\
\Pi_{\gamma \gamma}\left(q^{2}\right) & =q^{2} \Pi_{\gamma \gamma}^{\prime}(0)
\end{aligned}
$$

where $\Pi_{Z \gamma}(0)=\Pi_{\gamma \gamma}(0)=0$. Of the six parameters on the right-hand sides of these equations, we can absorb a linear combination three of them into the measured $\alpha_{\mathrm{EM}}, G_{F}$, and $m_{Z}$ through renormalization. There are then three orthogonal linear 


\begin{tabular}{c|c|ccc}
\hline \hline & Fit result & \multicolumn{3}{|c}{ Correlations } \\
\hline$S$ & $0.03 \pm 0.10$ & \multicolumn{2}{|c}{1} & \\
$T$ & $0.05 \pm 0.12$ & 0.89 & 1 & \\
$U$ & $0.03 \pm 0.10$ & -0.54 & -0.83 & 1 \\
\hline \hline
\end{tabular}

TABLE 5.2. Best-fit values for the electroweak oblique parameters from a global fit of electroweak data [165. The correlations indicate the interdependence of the measurements. Along the diagonal, each measurement is correlated with itself, then $S$ and $T$ are $89 \%$ correlated, and $U$ is anti-correlated with both $S$ and $T$.

combinations that can be used to probe the self-energies. These combinations are defined as

$$
\begin{aligned}
& \alpha_{\mathrm{EM}} S=4 s_{W}^{2} c_{W}^{2}\left[\Pi_{Z Z}^{\prime}(0)-\frac{c_{W}^{2}-s_{W}^{2}}{s_{W} c_{W}} \Pi_{Z \gamma}^{\prime}(0)-\Pi_{\gamma \gamma}^{\prime}(0)\right], \\
& \alpha_{\mathrm{EM}} T=\frac{\Pi_{W W}(0)}{m_{W}^{2}}-\frac{\Pi_{Z Z}(0)}{m_{Z}^{2}}, \\
& \alpha_{\mathrm{EM}} U=4 s_{W}^{2}\left[\Pi_{W W}^{\prime}(0)-c_{W}^{2} \Pi_{Z Z}^{\prime}(0)-2 s_{W} c_{W} \Pi_{Z \gamma}^{\prime}(0)-s_{W}^{2} \Pi_{\gamma \gamma}^{\prime}(0)\right] .
\end{aligned}
$$

The parameters $S, T$, and $U$ are known as the oblique parameters or the PeskinTakeuchi parameters [164].

Written in this way, we can relate back to the physical parameters such as the masses of the $W$ and $Z$ bosons,

$$
m_{Z}^{2}=\hat{m}_{Z}^{2} \frac{1-\alpha_{\mathrm{EM}} T}{1-G_{F} \hat{m}_{Z}^{2} \frac{S}{\sqrt{8 \pi^{2}}}} \quad, \quad m_{W}^{2}=\hat{m}_{W}^{2} \frac{1}{1-G_{F} \hat{m}_{W}^{2} \frac{(S+U)}{\sqrt{8 \pi^{2}}}}
$$

where, again, $\hat{m}$ is the SM predicted value. Notice that if $S=T=U=0, m=\hat{m}$. Measurements of $S, T$, and $U$ are given in Table 5.2. The close agreement with the SM value of zero allows us to constrain the parameters $\lambda_{3}$ and $\lambda_{4}$ through the mixing 

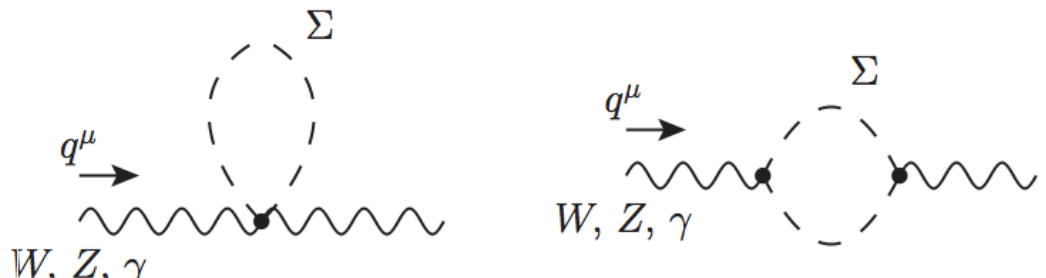

Figure 5.9. Feynman diagrams showing the one-loop contributions of the large multiplets to the gauge boson propagators. $\Sigma$ represents any valid multiplet member that can contribute.

angle $\alpha_{Q}$ and the masses of the states $H_{k}^{Q}$, since the oblique parameters depend on the NP masses and their couplings to SM gauge bosons.

The parameters $S, T$, and $U$ were previously calculated for a large scalar multiplet with arbitrary isospin and hypercharge in the case of a global U(1) symmetry in Ref. [166]. We recomputed the contributions of a large scalar multiplet to $S, T$, and $U$ for the global $Z_{2}$-preserving case, in which the mass eigenstates do not always correspond to isospin eigenstates.

For the $S$ parameter we find,

$$
S=\frac{2 s_{W}^{2} c_{W}^{2}}{\pi} \sum_{i, j}\left(\left|C_{i j Z}\right|^{2}-\frac{c_{W}^{2}-s_{W}^{2}}{s_{W} c_{W}} C_{i j Z} C_{i j \gamma}^{*}-\left|C_{i j \gamma}\right|^{2}\right) f_{1}\left(m_{i}, m_{j}\right)
$$

where the couplings $C_{i j V}$ involving scalars $i, j$ and vector boson $V$ are defined with an overall factor of $e$ removed (see Appendix A.2). The sums over states $i$ and $j$ run over $i j=\left\{\zeta^{0, r} \zeta^{0, i}, H_{k}^{+Q} H_{l}^{-Q}, \zeta^{n / 2} \zeta^{-n / 2}\right\}$, where $k l=11,12,21,22$ and $Q>0$. The 
dimensionless function $f_{1}\left(m_{1}, m_{2}\right)$ is defined as

$$
\begin{aligned}
& f_{1}\left(m_{1}, m_{2}\right)=f_{1}\left(m_{2}, m_{1}\right)=\int_{0}^{1} d x x(1-x) \log \left[x m_{1}^{2}+(1-x) m_{2}^{2}\right] \\
& =\left\{\begin{array}{lc}
\frac{1}{3} \log m, & m_{1}=m_{2} \equiv m \\
\frac{m_{1}^{6}-3 m_{1}^{4} m_{2}^{2}}{3\left(m_{1}^{2}-m_{2}^{2}\right)^{3}} \log m_{1}+\frac{m_{2}^{6}-3 m_{2}^{4} m_{1}^{2}}{3\left(m_{2}^{2}-m_{1}^{2}\right)^{3}} \log m_{2}-\frac{5 m_{1}^{4}-22 m_{1}^{2} m_{2}^{2}+5 m_{2}^{4}}{36\left(m_{1}^{2}-m_{2}^{2}\right)^{2}}, & m_{1} \neq m_{2}
\end{array} .\right.
\end{aligned}
$$

For the $T$ parameter we find,

$$
\begin{aligned}
T=\frac{1}{4 \pi m_{Z}^{2}} & {\left[-\sum_{r} S_{r}\left(\frac{C_{r r^{*} W^{+} W^{-}}}{c_{W}}-C_{r r^{*} Z Z}\right) f_{2}\left(m_{r}, m_{r}\right)\right.} \\
& \left.-2 \sum_{s, t} \frac{\left|C_{s t W^{+}}\right|^{2}}{c_{W}} f_{2}\left(m_{s}, m_{t}\right)+2 \sum_{i, j}\left|C_{i j k}\right|^{2} f_{2}\left(m_{i}, m_{j}\right)\right]
\end{aligned}
$$

where the couplings $C_{r r^{*} X Y}$ involving scalars $r r^{*}$ and vector bosons $X, Y$ are defined with an overall factor of $e^{2}$ removed (see Appendix A.2). The sum over states $r$ runs over $r=\left\{\zeta^{0, r}, \zeta^{0, i}, H_{k}^{Q}, \zeta^{n / 2}\right\}$, with $Q>0$ and $k=1,2$. For these couplings, $S_{r}$ is a symmetry factor given by $S_{r}=1 / 2$ for $r=\zeta^{0, r}$ or $\zeta^{0, i}$ and $S_{r}=1$ otherwise. The sums over states $s$ and $t$ run over $s t=\left\{\zeta^{0, r} H_{k}^{-}, \zeta^{0, i} H_{k}^{-}, H_{k}^{+Q} H_{l}^{-Q-1}, \zeta^{n / 2} H_{k}^{-n / 2-1}\right\}$, where again $k=1,2, k l=11,12,21,22$, and $Q>0$. The sums over states $i$ and $j$ run over the same set of states given below Equation (5.11).

The function $f_{2}\left(m_{1}, m_{2}\right)$ has dimensions of mass-squared and is defined as

$$
\begin{aligned}
f_{2}\left(m_{1}, m_{2}\right) & =f_{2}\left(m_{2}, m_{1}\right)=\int_{0}^{1} d x\left(x m_{1}^{2}+(1-x) m_{2}^{2}\right) \log \left[x m_{1}^{2}+(1-x) m_{2}^{2}\right] \\
& =\left\{\begin{array}{ll}
2 m^{2} \log m, & m_{1}=m_{2} \equiv m \\
\frac{m_{1}^{4}}{m_{1}^{2}-m_{2}^{2}} \log m_{1}+\frac{m_{2}^{4}}{m_{2}^{2}-m_{1}^{2}} \log m_{2}-\frac{1}{4}\left(m_{1}^{2}+m_{2}^{2}\right) & m_{1} \neq m_{2}
\end{array} .\right.
\end{aligned}
$$


For the $U$ parameter we find,

$$
\begin{aligned}
U=\frac{s_{W}^{2}}{\pi} & {\left[\sum_{s, t}\left|C_{s t W^{+}}\right|^{2} f_{1}\left(m_{s}, m_{t}\right)\right.} \\
& \left.-\sum_{i, j}\left(c_{W}^{2}\left|C_{i j Z}\right|^{2}+2 s_{W} c_{W} C_{i j Z} C_{i j \gamma}^{*}+s_{W}^{2}\left|C_{i j \gamma}\right|^{2}\right) f_{1}\left(m_{i}, m_{j}\right)\right],
\end{aligned}
$$

where the sums over states $i j$ and st run over the same sets of states given below Equations (5.11) and (5.13),

We impose a $95 \%$ confidence level constraint from the oblique parameters using a $\chi^{2}$ built from the experimental values given in Table 5.2. The $95 \%$ confidence level $\chi^{2}$ constraint in the 3 -dimensional parameter space is given by ${ }^{3}$

$$
\chi^{2}=\sum_{i, j}\left(\mathcal{O}_{i}-\mathcal{O}_{i}^{\exp }\right)\left(\mathcal{O}_{j}-\mathcal{O}_{j}^{\exp }\right)\left[\sigma^{2}\right]_{i j}^{-1} \leq 7.815
$$

where $\mathcal{O}_{i}$ is the $i$ th observable of the set $S, T, U$ and $\left[\sigma^{2}\right]_{i j}^{-1}$ is the inverse of the matrix of uncertainties,

$$
\left[\sigma^{2}\right]_{i j}=\Delta \mathcal{O}_{i} \Delta \mathcal{O}_{j} \rho_{i j}
$$

where $\rho_{i j}$ are the relative correlations (note that $\rho_{i i} \equiv 1$ ).

The contributions of the large multiplet to $S, T$, and $U$ depend only on the mass spectrum and mass-eigenstate compositions. Thus the oblique parameters constrain only $\lambda_{3}, \lambda_{4}$, and the overall mass scale, which can be parameterized by $m_{\zeta^{0, r}}$. The effects of the $S T U$ constraints on the parameter space in the $\lambda_{3} \lambda_{4}$-plane is shown ${ }^{3}$ We use a $\chi^{2}$ constraint, rather than a $\Delta \chi^{2} \equiv \chi^{2}-\chi_{\min }^{2}$ relative to the minimum $\chi^{2}$ obtained in the model, so that parameter points that yield $S, T, U$ values too far from the experimental measurements will be excluded. For comparison, the SM point $(S, T, U)=(0,0,0)$ has a $\chi^{2}$ value of 4.40 and the minimum $\chi^{2}$ obtained in the models is about 1.4 for $n=6$ and 1.3 for $n=8$. 

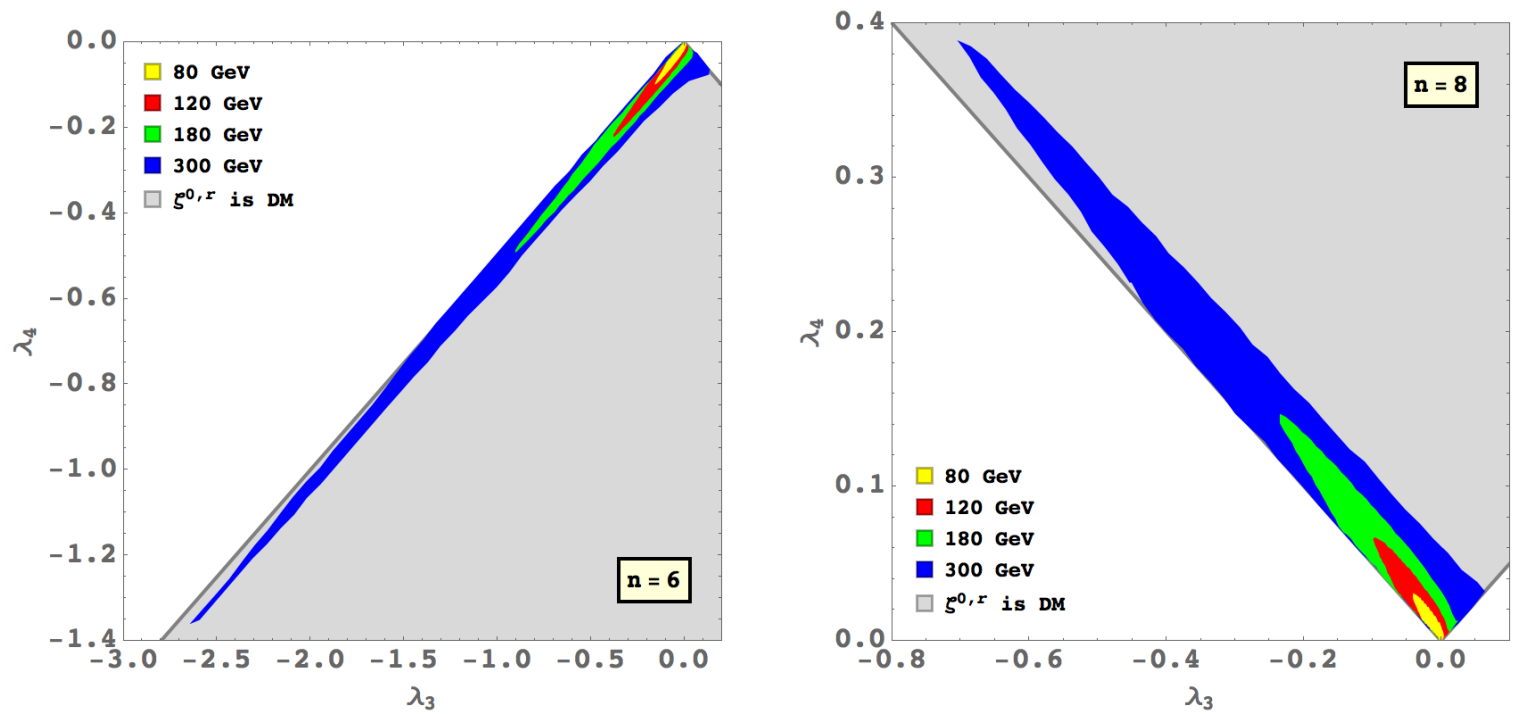

Figure 5.10. Allowed regions in the $\lambda_{3} \lambda_{4}$-plane with $S T U$ bounds imposed. The yellow region corresponds to the points at $m_{\zeta^{0, r}}=80 \mathrm{GeV}$, red to $m_{\zeta^{0, r}}=120 \mathrm{GeV}$, green to $m_{\zeta^{0, r}}=180 \mathrm{GeV}$, and blue to $m_{\zeta^{0, r}}=300 \mathrm{GeV}$. The grey region is the required region for $\zeta^{0, r}$ to be the DM $\left(\left|\lambda_{3}\right| \leq 2\left|\lambda_{4}\right|\right)$. The unitarity bounds from Table 4.2 would scale the plots such that the low- $m_{\zeta^{0, r}}$ regions would not be visible.

in Figure 5.10, where we have imposed the additional DM constraint of $\left|\lambda_{3}\right| \leq 2\left|\lambda_{4}\right|$. From this, we see that small values of $\left(\lambda_{3}, \lambda_{4}\right)$ are allowed, with $\left|\lambda_{3}\right| \approx 2\left|\lambda_{4}\right|$.

We also plot the effect of $S T U$ constraints on the overall mass splitting in Figure 5.11. While the $S T U$ bound (dashed black line) is stronger than the unitarity bound (dotted red curve) at low- $m_{\zeta^{0, r}}$, it becomes weaker as the mass increases until the unitarity bound is the dominant constraint once $m_{\zeta^{0, r}} \gtrsim 530 \mathrm{GeV}(809 \mathrm{GeV})$ in the $n=6(8)$ model.

\subsection{2. $h \rightarrow \gamma \gamma$.}

The SM Higgs boson is not a stable particle. Its decay width is predicted to be $\sim 4 \mathrm{MeV}$, which corresponds to a lifetime of $\sim 10^{-21} \mathrm{~s}$ (for comparison, the lifetime of a $Z$ boson is $\sim 10^{-25} \mathrm{~s}$, while that of a muon is $\sim 10^{-6} \mathrm{~s}$, and that of a neutron 

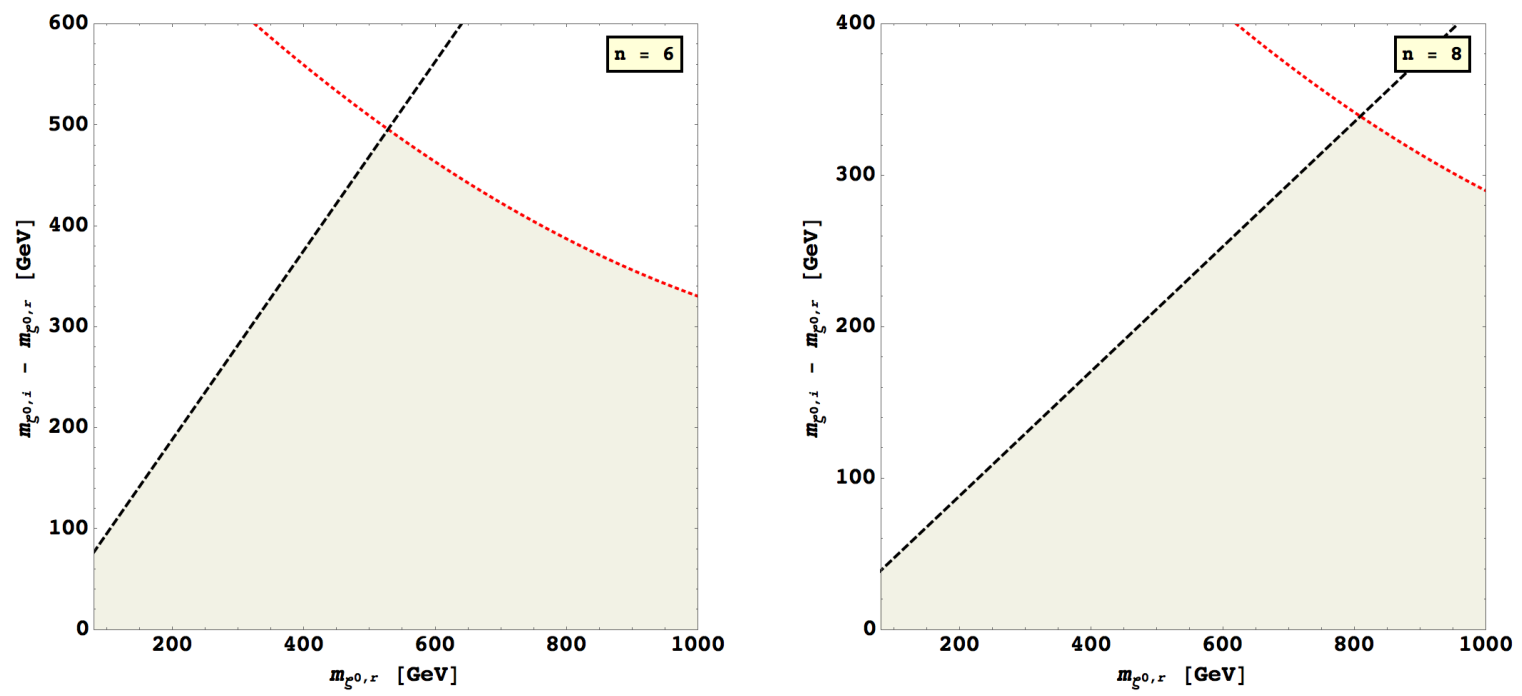

FiguRE 5.11. Mass splitting between $\zeta^{0, r}$ and $\zeta^{0, i}$ with both unitarity and $S T U$ bounds imposed. The plot on the left is for $n=6$ and the plot on the right is for $n=8$. The shaded region is that which is allowed by both constraints. The dotted red curve shows the upperbound coming from unitarity Table 4.2, and the dashed black line shows the upper-bound coming from the $S T U$ constraints.

is $\sim 15$ minutes). This means that we cannot observe the Higgs directly, but rather only its decay products.

One of the most important discovery modes of the Higgs boson was through its decay to two photons, yet it does not couple directly to photons (its electric charge is zero). How can this be? Processes in the SM do not occur only via tree-level diagrams, they will also proceed through loops of particles. In the SM, the decay $h \rightarrow \gamma \gamma$ will proceed through loops containing all the particles that couple to the Higgs and carry electric charge. In the SM, the coupling of a particle to the Higgs is proportional to its mass, which means that the most important contributions to this decay will come from the heaviest charged particles: the top quark and the $W$ boson. Contributions from lighter SM states will only serve to tighten the constraints slightly. The relevant diagrams at one loop are shown in Figure 5.12. 

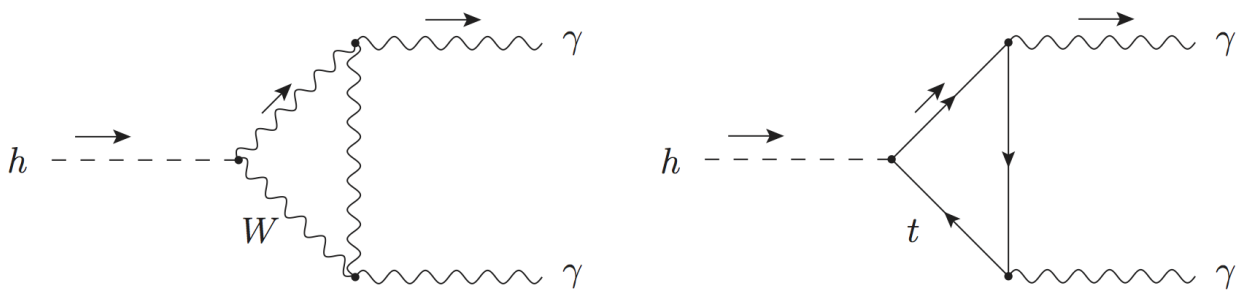

Figure 5.12. Sample Feynman diagrams showing the most important SM contributions to $h \rightarrow \gamma \gamma$. The $W$ boson and the $t$ quark are the most massive (electrically charged) particles of each type, thus their couplings to the Higgs are the strongest.

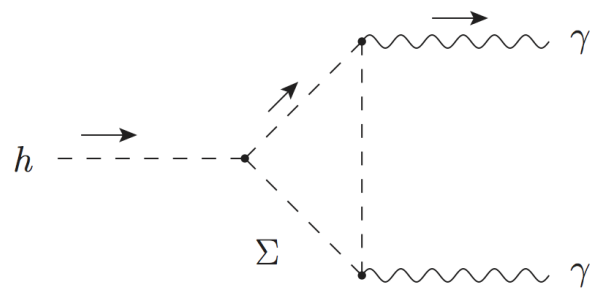

FiguRE 5.13. Sample Feynman diagrams showing the large multiplet contributions to $h \rightarrow \gamma \gamma$. In this case, $\Sigma$ represents any electricallycharged member of the large multiplet.

Recall that the scalar potential, Equation (3.4), couples particles from $\Sigma$ to the SM Higgs doublet, and that the charged scalars from $\Sigma$ will also couple to photons. That means that they will contribute to the process $h \rightarrow \gamma \gamma$, shown in Figure 5.13.

To constrain the parameter $\lambda_{2}$, we consider the one-loop contributions of the charged scalars to the Higgs decay $\rfloor^{4} h \rightarrow \gamma \gamma$. The decay partial width for $h \rightarrow \gamma \gamma$ can be written as [169],

$$
\Gamma(h \rightarrow \gamma \gamma)=\frac{\alpha_{\mathrm{EM}}^{2} g^{2}}{1024 \pi^{3}} \frac{m_{h}^{3}}{m_{W}^{2}}\left|\sum_{i} N_{c i} Q_{i}^{2} F_{i}\right|^{2}
$$

where $i$ sums over charged particles of spin $0,1 / 2$, and $1, Q_{i}$ is the electric charge of the particle in the loop in units of $e, N_{c i}$ is the color multiplicity ( 3 for quarks and 1 ${ }^{4}$ Contributions to $h \rightarrow \gamma \gamma$ from multiply-charged scalars in the loop have also been considered in Refs. [167, 168. 
for color-singlet particles) and $F_{i}$ is a function that depends on the spin and mass of the particle in the loop [169]:

$$
\begin{aligned}
F_{1} & =2+3 \tau+3 \tau(2-\tau) f(\tau), \\
F_{1 / 2} & =-2 \tau[1+(1-\tau) f(\tau)], \\
F_{0} & =\beta \tau[1-\tau f(\tau)] .
\end{aligned}
$$

Here $\tau_{i}=4 m_{i}^{2} / M_{h}^{2}$, where $m_{i}$ is the mass of the particle in the loop, and the function $f(\tau)$ is given by $[\mathbf{1 6 9}$,

$$
f(\tau)= \begin{cases}{\left[\arcsin \left(\sqrt{\frac{1}{\tau}}\right)\right]^{2}} & \tau \geq 1 \\ -\frac{1}{4}\left[\log \left(\frac{\eta_{+}}{\eta_{-}}\right)-i \pi\right]^{2} & \tau<1\end{cases}
$$

with $\eta_{ \pm} \equiv 1 \pm \sqrt{1-\tau}$. In the numerical computation of partial widths we include only the top quark and $W$ boson contributions, as well as the new scalars; contributions from the lighter fermions are small.

For a scalar particle in the loop, we have inserted the factor $\beta$ into the definition of $F_{0}$ to capture the coupling of the scalar to the Higgs,

$$
\beta=C_{h s s} \frac{m_{W}}{g m_{s}^{2}}=C_{h s s} \frac{v}{2 m_{s}^{2}} .
$$

The couplings $C_{h s s}$ for our models are collected in Appendix A. Note that all couplings of the Higgs to pairs of scalars from the large multiplet are diagonal in the mass basis. The functions $\beta$ for the scalars $H_{i}^{Q}$ and $\zeta^{+n / 2}$ in the loop can be written as

$$
\begin{aligned}
\beta_{H_{1,2}^{Q}} & =\frac{v^{2}}{2 m_{H_{1,2}^{2}}^{2}}\left[\lambda_{2}+\frac{\lambda_{3}}{4} \mp \frac{1}{2} \sqrt{Q^{2} \lambda_{3}^{2}+\left(n^{2}-4 Q^{2}\right) \lambda_{4}^{2}}\right], \\
\beta_{\zeta^{+n / 2}} & =\frac{v^{2}}{2 m_{\zeta^{+n / 2}}^{2}}\left[\lambda_{2}-\frac{n-1}{4} \lambda_{3}\right] .
\end{aligned}
$$




\begin{tabular}{c|cc}
\hline \hline Observable & ATLAS & CMS \\
\hline$\mu_{\gamma \gamma}$ & $1.65_{-0.30}^{+0.32}[\mathbf{1 7 0}]$ & $0.78_{-0.26}^{+0.28}[\mathbf{1 7 1}]$ \\
$\mu_{Z \gamma}$ & $<18.2[\mathbf{1 7 2}]$ & $<10[\mathbf{1 7 3}]$ \\
\hline
\end{tabular}

TABLE 5.3. Measurements of the signal strengths for $h \rightarrow \gamma \gamma$ and $h \rightarrow Z \gamma$ relative to the SM predictions. For $h \rightarrow Z \gamma$ we quote the $95 \%$ confidence level upper bounds for $m_{h}=125 \mathrm{GeV}$.

Because these couplings depend on $\lambda_{2}$, measurements of the Higgs decay $h \rightarrow \gamma \gamma$ can be used to put additional constraints on $\lambda_{2}$ as a function of $\lambda_{3}, \lambda_{4}$, and $m_{\zeta^{0, r}}$. The ATLAS and CMS experiments have measured the Higgs signal strength $\mu_{\gamma \gamma}$ in the $\gamma \gamma$ final state, defined relative to the SM prediction. Because Higgs production rates are not modified in our models, and because the only significant effect of the new scalars on Higgs decays is through modification of the partial widths of the rare loop-induced processes $h \rightarrow \gamma \gamma$ and $h \rightarrow Z \gamma$, we have to a very good approximation

$$
\mu_{\gamma \gamma} \simeq R_{\gamma \gamma} \equiv \frac{\Gamma(h \rightarrow \gamma \gamma)}{\Gamma_{\mathrm{SM}}(h \rightarrow \gamma \gamma)}
$$

and an analogous expression for $R_{Z \gamma}$. The measured values of this rate from ATLAS and CMS are summarized in Table 5.3.

We find the allowed range of $\lambda_{2}$ as a function of $m_{\zeta^{0, r}}$ in two cases. First, we scan over the values of $\lambda_{2,3,4}$ allowed by perturbative unitarity (see Table 4.2), and calculate Equation (5.18), rejecting those points which fall outside the union of the $2 \sigma$ ranges for $\mu_{\gamma \gamma}$ of the ATLAS and CMS measurements. The regions which satisfy these constraints are shown in blue and green in Figure 5.14. Next, we include the constraints coming from the oblique parameters, $S T U$. The regions allowed by the combination of unitarity, $S T U$, and $h \rightarrow \gamma \gamma$ are shown in green in Figure 5.14. The region excluded by perturbative unitarity, $\left|\lambda_{2}\right| \leq 6.59$ (3.10) for $n=6(8)$, is is shown 

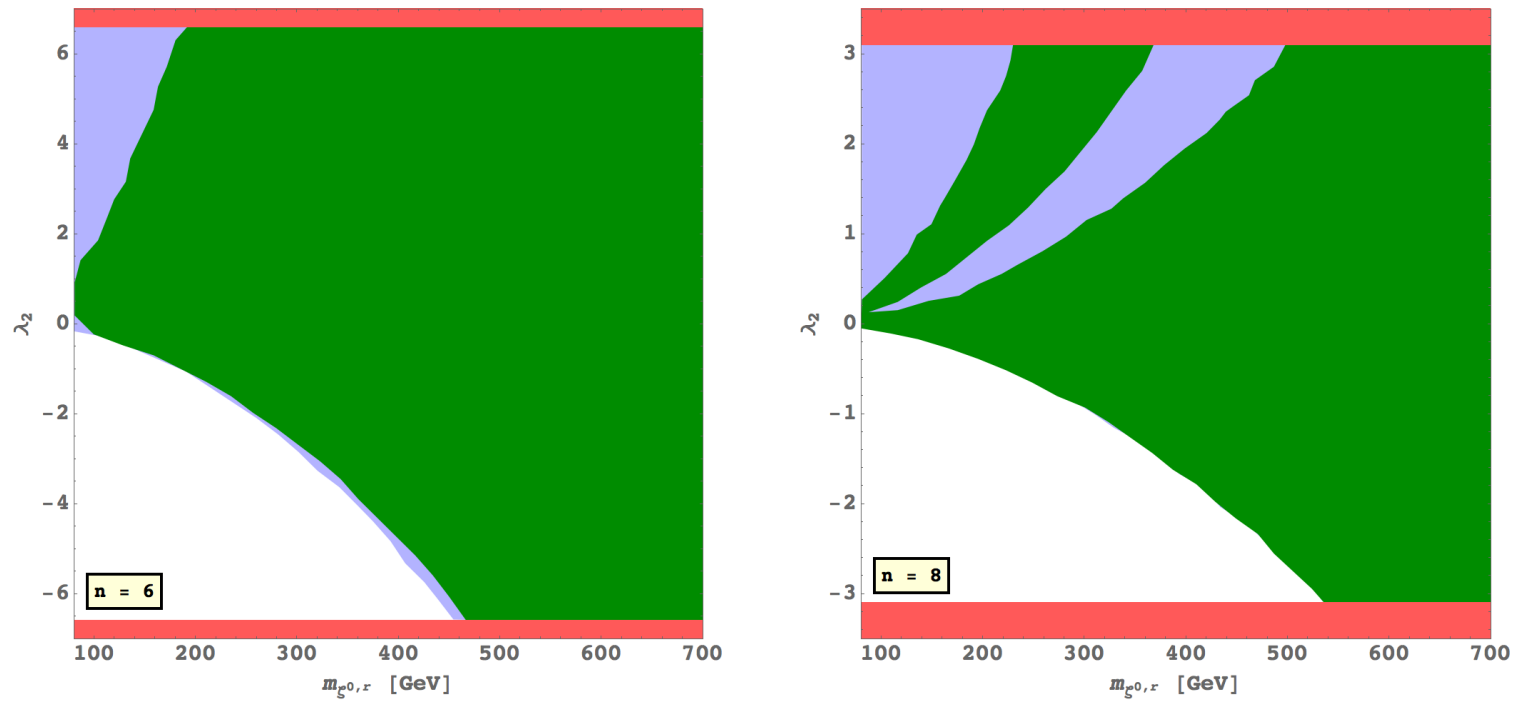

Figure 5.14. Allowed $\lambda_{2}$ values as a function of $m_{\zeta^{0, r}}$ with $S T U$ and $h \rightarrow \gamma \gamma$ constraints imposed. The (darker) green region shows those points allowed by both $S T U$ and $h \rightarrow \gamma \gamma$ constraints. The (lighter) blue region shows those points allowed only by $h \rightarrow \gamma \gamma$. The red regions are the bounds imposed by unitarity from Table 4.2 .

in red in these plots. The upper branch of allowed $\lambda_{2}$ values, clearly visible in the green region in the right panel of Figure 5.14 for $n=8$, corresponds to a sign flip of the total $h \rightarrow \gamma \gamma$ amplitude relative to the SM prediction. This is separated from the rest of the points due to the lower bound on $\mu_{\gamma \gamma}$. The same feature does not appear in the $n=6$ case (the left-hand plot of Figure 5.14 due to the wider allowed ranges of $\lambda_{3}$ and $\lambda_{4}$.

The application of the $h \rightarrow \gamma \gamma$ constraint does not significantly restrict the range of $\lambda_{3}$ or $\lambda_{4}$ beyond the constraints already obtained from the oblique parameters and perturbative unitarity.

\subsection{3. $h \rightarrow Z \gamma$.}

Another possibility for the loop-induced decay of the Higgs is $h \rightarrow Z \gamma$ (other processes will occur at tree-level or not at all). Overall, the structure of these decays 


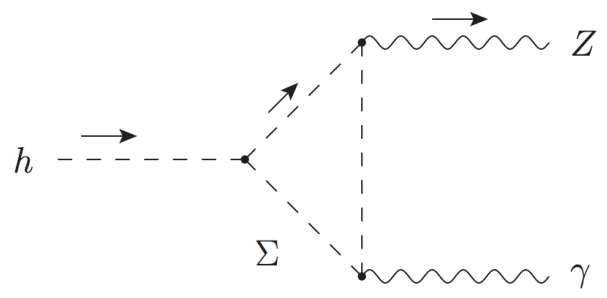

Figure 5.15. Feynman diagrams showing the large multiplet contributions to $h \rightarrow Z \gamma$. In this case, $\Sigma$ represents any member of the large multiplet.

will be similar to $h \rightarrow \gamma \gamma$, but with a different coupling, the resulting expressions will be necessarily more complex. In addition, subsequent decays of the $Z$ may be difficult to detect (buried in background or invisible in the case of neutrinos). The constraint will then be less restrictive than in the $h \rightarrow \gamma \gamma$ case; indeed, the current ATLAS and CMS searches for the mode only place an upper-bound on the rate of ten times the SM prediction (see Table 5.3). The decay partial width for this process can be written as (see, e.g., [169])

$$
\Gamma(h \rightarrow Z \gamma)=\frac{\alpha_{\mathrm{EM}}^{2}}{512 \pi^{3}}\left|\frac{2}{v}\left(A_{F}+A_{W}\right)+A_{S}\right|^{2} m_{h}^{3}\left[1-\frac{m_{Z}^{2}}{m_{h}^{2}}\right]^{3},
$$


The contributions to the amplitude from fermions, the $W$ boson, and scalars are given by $5^{5}$

$$
\begin{gathered}
A_{F}=\sum_{f} N_{c f} \frac{-2 Q_{f}\left(T_{f}^{3 L}-2 Q_{f} s_{W}^{2}\right)}{s_{W} c_{W}}\left[I_{1}\left(\tau_{f}, \lambda_{f}\right)-I_{2}\left(\tau_{f}, \lambda_{f}\right)\right], \\
A_{W}=-\cot \theta_{W}\left\{4\left(3-\tan ^{2} \theta_{W}\right) I_{2}\left(\tau_{W}, \lambda_{W}\right)\right. \\
\left.+\left[\left(1+\frac{2}{\tau_{W}}\right) \tan ^{2} \theta_{W}-\left(5+\frac{2}{\tau_{W}}\right)\right] I_{1}\left(\tau_{W}, \lambda_{W}\right)\right\}, \\
A_{S}=2 \sum_{s} \frac{C_{h s s} C_{s s Z} Q_{s}}{m_{s}^{2}} I_{1}\left(\tau_{s}, \lambda_{s}\right),
\end{gathered}
$$

where the scalar couplings $C_{h s s}$ and $C_{s s}$ are given in Appendix $\mathrm{A}$ and $\lambda_{i}$ is defined analogously to $\tau_{i}$ but with $m_{h}$ replaced by $m_{Z}$ :

$$
\lambda_{i}=\frac{4 m_{i}^{2}}{m_{Z}^{2}} .
$$

The sums over $f$ and $s$ run over all charged fermions and scalars in the model (in our numerical calculation, we neglect all fermions other than the top quark).

The functions $I_{i}(a, b)$ are defined as 169

$$
\begin{aligned}
& I_{1}(a, b)=\frac{a b}{2(a-b)}+\frac{a^{2} b^{2}}{2(a-b)^{2}}[f(a)-f(b)]+\frac{a^{2} b}{(a-b)^{2}}[g(a)-g(b)], \\
& I_{2}(a, b)=-\frac{a b}{2(a-b)}[f(a)-f(b)],
\end{aligned}
$$

${ }^{5}$ It was pointed out in Ref. [174 that there are some discrepancies in the literature among different calculations of the contributions of charged scalars to the amplitude for $h \rightarrow Z \gamma$. Our formulas are consistent with those of Refs. [169, 174, 175. 
where the function $f(\tau)$ was given in Equation (5.20) and the function $g(\tau)$ is defined as

$$
g(\tau)= \begin{cases}\sqrt{\tau-1} \arcsin \left(\sqrt{\frac{1}{\tau}}\right) & \text { if } \tau \geq 1, \\ \frac{1}{2} \sqrt{1-\tau}\left[\log \left(\frac{\eta_{+}}{\eta_{-}}\right)-i \pi\right] & \text { if } \tau<1 .\end{cases}
$$

The LHC experiments currently constrain the rate for $h \rightarrow Z \gamma$ to be less than about 10 times the SM prediction (see Table 5.3). This does not further constrain the parameter space of our models once the constraints from perturbative unitarity, oblique parameters, and $h \rightarrow \gamma \gamma$ have been applied.

\subsection{Chapter summary}

In this chapter, we examined how experimental searches for physics beyond the SM and precise measurements of SM processes constrain the parameter space of the large multiplet models. We began with direct searches for NP performed by the ATLAS and CMS collaborations at the LHC. Using the results of these searches, we found that the low- $m_{\zeta^{0, r}}$ regions of parameter space $\left(m_{\zeta^{0, r}}=80,120 \mathrm{GeV}\right)$ are mostly excluded in both the $n=6$ and 8 models, and while there is some exclusion at $m_{\zeta^{0, r}}=180 \mathrm{GeV}$ in $n=6$, the higher mass regions remain unconstrained by the LHC searches. These results were originally published in Ref. [3].

We also examined the effects our model will have on SM processes at the loop level. The strongest constraints in this regard come from the electroweak precision observables, $S T U$, which constrain the values of $\lambda_{3,4}$ such that $\lambda_{3} \approx-2\left|\lambda_{4}\right|$ unless they are both small. The rate of Higgs decays to two photons relative to the SM prediction give some constraint to $\lambda_{2}$, but the upper-bound on the rate for decays to

a $Z$ boson and a photon are too loose to provide any further constraint beyond the unitarity bound. These results were originally published in Ref. [2]. 


\section{CHAPTER 6}

\section{Dark matter constraints on $Z_{2}$-symmetric models}

Although the previous chapters held, in the background, that our models contained a dark matter (DM) candidate, only two constraints were derived from this requirement. The first was that the multiplet did not acquire a vacuum expectation value, and the second was in choosing one of the neutral components to be the lightest member, resulting in $\left|\lambda_{3}\right| \leq 2\left|\lambda_{4}\right|$. The other constraints we derived-perturbative unitarity, stability of the potential, direct collider, and loop-induced constraintscould just as easily be applied to any model of beyond the Standard Model (BSM) physics. Recall Figure 1.6, in which is described the generic interaction of two DM particles with two SM particles.

Chapter 5 used the production $(\uparrow)$ arrow in its direct searches and (indirectly) the annihilation $(\downarrow)$ in the loop-induced processes. In this chapter, we will use both the production and annihilation arrows $(\uparrow)$ to determine the amount of DM produced by our models in the early Universe - its relic abundance. We will also use the scattering $(\leftrightarrow)$ arrow to determine the prospects for direct detection experiments.

When we examine the parameter space of our models, we find two somewhat overlapping regions of parameter values based on the mass of the DM candidate, $m_{\zeta^{0, r}}$. In the weak-scale $\mathrm{DM}$ mass region, $80 \mathrm{GeV} \lesssim m_{\zeta^{0, r}} \lesssim 1 \mathrm{TeV}$, there are different effects at play in the determination of the relic abundance than in the multiTeV DM mass region, $1 \mathrm{TeV} \lesssim m_{\zeta^{0, r}} \lesssim 100 \mathrm{TeV}$. The bounds from Chapters 4 and 5 will provide more stringent constraints on the weak-scale region, meaning that $\zeta^{0, r}$ 
will not be able to constitute all of the DM. In the multi-TeV region, the constraints are much weaker, and so we are interested in constraints arising from demanding that $\zeta^{0, r}$ be all of the DM.

For this reason, we will describe the results for the two regions separately. The remainder of this chapter will be divided as follows. First, we will give an overview of the standard thermal history of the Universe and how DM particles freeze out in the early Universe. We will then describe the effect of our previously constrained parameter space on the calculation of the DM relic abundance in the weak-scale region. Next, we will describe how co-annihilation and Sommerfeld enhancement will affect the relic abundance of $\zeta^{0, r}$ in the multi-TeV range, and the resulting constraints from demanding $\zeta^{0, r}$ be all of the DM. We then move on to discuss how DM may be detected directly on the surface (or, more precisely, below the surface) of Earth. Finally, we apply our constrained parameter space to the calculation of the DM-nucleon scattering cross section and determine the discovery prospects of both the weak-scale and multi-TeV DM mass regions at current and future DM search experiments.

\subsection{Thermal history of the Universe}

The Universe that we now see is not the same as it has always been. Measurements of galaxy spectra all across the sky show a nearly unanimous redshift, indicating that they are moving away from us. Turning this around, this means that at some point in the past, distant parts of the Universe were closer together. Taken to its extreme, all parts of the Universe were, at one time, together in a singularity. The change from singularity to expansion is what is known as the Big Bang.

When we go to count the galaxies around us, we find that - although their number and density may vary - they are remarkably evenly distributed in our skies. This 
indicates that, for at least the part of it that we see, our Universe is isotropic (the same in every direction) and homogenous (the same at every point) on large scales. If we want to describe the spacetime of an expanding, spatially isotropic and homogenous Universe, we would use a Friedmann-Lemaître-Robertson-Walker (FLRW) metric [17, 176],

$$
d s^{2}=d t^{2}-a^{2}(t)\left[\frac{d r^{2}}{1-k r^{2}}+r^{2} d \theta^{2}+r^{2} \sin \theta d \phi^{2}\right],
$$

where $k \in\{-1,0,+1\}$ describes the curvature of the spacetime and $a(t)$ is the timedependent scale factor. The expansion of the Universe is described by the Hubble parameter,

$$
H \equiv \frac{1}{a(t)} \frac{d a(t)}{d t}=\frac{\dot{a}}{a},
$$

which is usually given in terms of $H=(100 \mathrm{~km} / \mathrm{s} / \mathrm{Mpc}) h$. The current measurement of the Hubble parameter is $H_{0}=67.8 \pm 0.9 \mathrm{~km} / \mathrm{s} / \mathrm{Mpc}$ [25]. The age of the Universe may be determined from $t_{0}=H_{0}^{-1} \approx 13.7 \times 10^{9}$ years. We are bathed in black-body radiation with a temperature of $T_{0}=2.73 \mathrm{~K}$. The energy density of a black-body emitter is proportional to the fourth power of its temperature, $\rho \propto T^{4}$, while (for a relativistic species) it is also inversely proportional to the fourth power of the scale factor, $\rho \propto a^{-4}$. This means that the temperature is inversely proportional to the scale factor, $T \propto a^{-1}$. As the Universe shrinks back to a singularity $(a \rightarrow 0)$, the temperature will increase. On the other hand, as the Universe expands, the temperature will decrease. What can we learn from the evolution of the Universe? We now give a brief overview of the standard lore for the thermal history of the Universe [176, 177].

In the beginning, the Universe was infinitely hot and infinitely dense. At that time, it seems likely, all of the forces (including gravity) were unified. As it began 
to expand, the temperature dropped, breaking the symmetries present and inducing phase transitions. At a temperature $T_{P l} \sim 10^{31} \mathrm{~K} \sim 10^{18} \mathrm{GeV}\left(t \lesssim 10^{-36} \mathrm{~s}\right)$, the symmetry between gravity and the other forces was broken. All of the SM particles were strongly interacting in a plasma formed from all types of particles. Then there was a period of very fast expansion, called inflation, where the Universe grew in size by a factor $\sim 10^{26}$ between $10^{-36} \mathrm{~s}$ and $10^{-33} \mathrm{~s}$. Our multi-TeV DM freezes out after this epoch. At around $T_{E W P T} \sim 2 \times 10^{15} \mathrm{~K} \sim 200 \mathrm{GeV}\left(t \sim 10^{-10} \mathrm{~s}\right)$, electroweak symmetry will be broken, and particles such as the $W$ and $Z$ bosons will acquire their masses. At this point, quarks are still free, along with electrons, photons, and neutrinos. Our weak-scale DM freezes out at temperatures near the electroweak phase transition temperature. Once the temperature falls to the QCD phase transition temperature, $T_{Q C D} \sim 10^{12} \mathrm{~K} \sim 200 \mathrm{MeV}\left(t \sim 10^{-4} \mathrm{~s}\right)$, confinement will take over and quarks will bind together to form baryons. The lightest atomic nuclei will form when the temperature reaches $T_{H^{+}} \sim 10^{10} \mathrm{~K} \sim 1 \mathrm{MeV}(t \sim 1 \mathrm{~s})$. From this point on, we have strong experimental support (based on the light element abundances) for this picture of the thermal history. Electrons become bound to the nuclei once the temperature drops to the recombination temperature, $T_{\text {recomb. }} \sim 3000 \mathrm{~K} \sim 0.25 \mathrm{eV}$ $\left(t \sim 3 \times 10^{5} \mathrm{yr}\right)$. Then, photons no longer scatter off of the charged electrons, but rather stream freely through the Universe. This will create a surface of last scattering, which we observe today as the Cosmic Microwave Background (CMB) radiation with temperature $T_{0}=2.73 \mathrm{~K}\left(t_{0}=13.7 \times 10^{9} \mathrm{yr}\right)$. From the recombination temperature until today, no interesting particle physics occurs at cosmological scales, but we can still glean important information from the CMB.

Before the recombination temperature, $T_{\text {recomb. }}$, the plasma of baryons, electrons, and photons will be affected by two processes: the outward pressure from the elastic 
scattering of photons off of electrons (Thomson scattering), and the inward "pressure" from the gravitational attraction among the baryons. If these forces were in balance then, as the Universe expanded and cooled, the photons would have to travel a longer distance between the electrons, losing energy and allowing the Coulomb interaction to win out. In this way, small fluctuations in the matter density throughout the plasma, mainly caused by DM, will drive oscillations [178] (see also Ref. [179]). At $T_{\text {recomb. }}$ the phase of those oscillations is frozen into the CMB. The resulting variations in the temperature of the CMB from one point to another will give us information about the early Universe. We can measure the temperature over the whole sky, using experiments such as Cosmic Background Explorer (COBE, NASA, 1989-1993), Wilkinson Microwave Anisotropy Probe (WMAP, NASA, 2001-2010), and Planck (ESA, 2009-2013), to produce a map of the CMB temperature. We can then plot a spectrum as a function of the angular size of the variations.

The spectrum shown in Figure 6.1 provides us with certain key observations about the early Universe. The first is that the size of the variations is very small, $\Delta T / T \approx$ $\sqrt{5 \times 10^{-9} \mathrm{~K}^{2}} /(2.73 \mathrm{~K}) \approx 10^{-5}$ for the first peak, indicating that the energy density was remarkably homogenous at the surface of last scattering. This small inhomogeneity, however, accounts for all of the structure observed in the Universe today. The position of the first peak in Figure 6.1 gives a measure of the curvature of the Universe, and it is consistent with the position predicted by a flat Universe. That the second peak is lower than the first indicates the need for more baryons than are bound in stars. The third peak provides a measure of the density of DM in the Universe. From the current best-fit value, we find the dark matter density to be $\Omega_{\mathrm{DM}} h^{2}=0.1188$ and the baryon density to be $\Omega_{b} h^{2}=0.02230[\mathbf{2 5}$. How, then, do we obtain this value in our models of DM? 


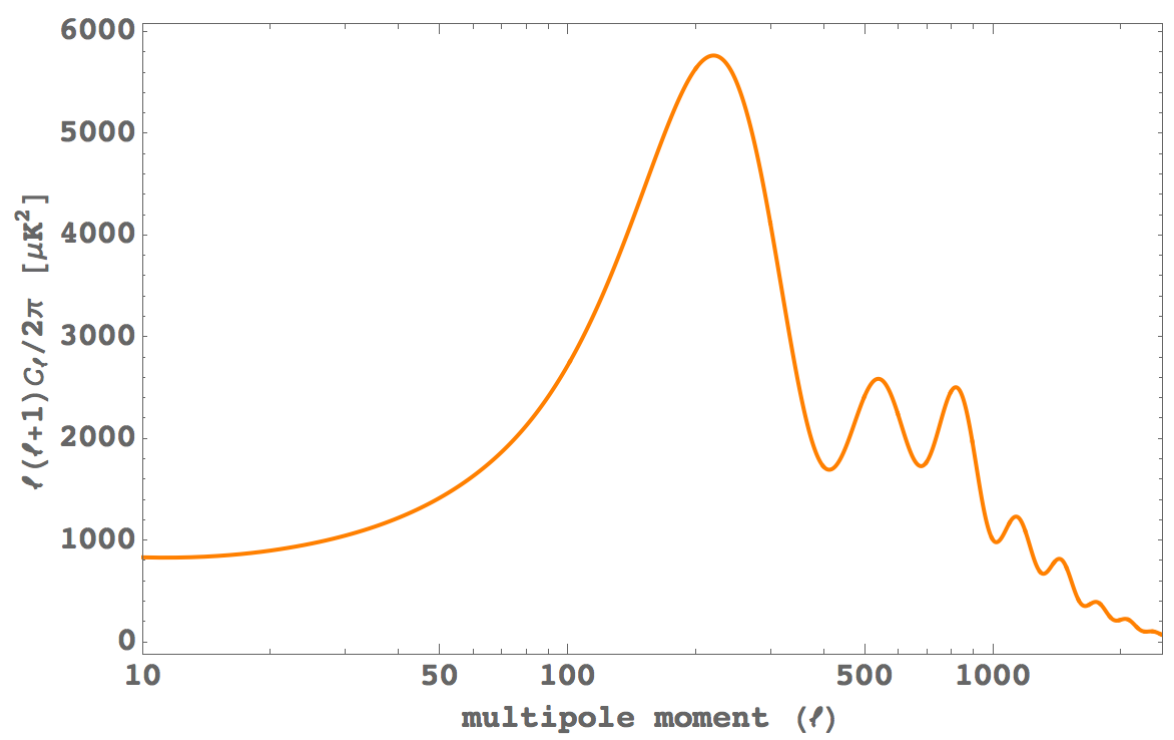

Figure 6.1. The CMB temperature power spectrum as a function of multipole, $\ell . \mathcal{C}_{\ell}=\frac{1}{2 \ell+1} \sum_{m=-\ell}^{\ell}\left\langle\left|a_{\ell m}\right|^{2}\right\rangle$ such that the temperature fluctuations are given by $\Theta=\sum_{\ell=0}^{\infty} \sum_{m=-\ell}^{\ell} a_{\ell m} Y_{\ell m}$. The ratio of the third peak $(\ell \sim 800)$ to the second peak $(\ell \sim 500)$ indicates the presence of DM in the Universe. Plot created using CMBquick (version 0.0.3) 180 with inputs from WMAP5 [181].

\subsection{Freeze-out}

The number density, $n$, of the particles of a gas with $g$ internal degrees of freedom is given in terms of its phase space distribution [182, 176], $f(\vec{p})$, by

$$
n=g \int \frac{d^{3} p}{(2 \pi)^{3}} f(\vec{p})
$$

For a non-relativistic species of mass $m$ at a temperature $T$ at zero chemical potential and in thermal equilibrium,

$$
n_{\mathrm{eq}}=g\left(\frac{m T}{2 \pi}\right)^{\frac{3}{2}} \mathrm{e}^{-\frac{m}{T}}
$$


Throughout the expansion of the Universe, the entropy per comoving volume is a constant,

$$
s=\frac{2 \pi^{2}}{45} g_{\star S} T^{3},
$$

where $g_{\star S}$ is the effective total number of relativistic degrees of freedom. We can then write the number density divided by the entropy density in the non-relativistic limit as

$$
Y_{\mathrm{eq}} \equiv \frac{n_{\mathrm{eq}}}{s}=\frac{45}{4 \sqrt{2} \pi^{5}} \frac{g}{g_{\star S}} x^{\frac{3}{2}} \mathrm{e}^{-x},
$$

where it is convenient to define $x=m / T$. If the species remained in thermal equilibrium to the present day ( $T \rightarrow T_{0} \sim 0$ ), then, as we see in the dashed black line in Figure 6.2, the number density divided by the entropy density will fall off to zero, $Y \sim 0$. However, if the interaction rate falls below the expansion rate, $\Gamma \lesssim H$, then the species will decouple from the plasma, the solid blue curve in Figure 6.2. That species will then have a significant relic abundance, also called its relic density. In these plots, $x$ acts as an analogue of time because $T$ falls monotonically with time. Notice that the dashed (in thermal equilibrium) curve drops to zero as "time" goes forward, while the solid (freeze-out) curve asymptotes to a constant value (its relic abundance).

To examine the freeze-out (or decoupling) of a particle species such as DM, we must use the Boltzmann equation, which relates the Liouville operator, $\widehat{\mathbf{L}}$, to the collision operator, $\widehat{\mathbf{C}}$. It is given by

$$
\widehat{\mathbf{L}}[f]=\widehat{\mathbf{C}}[f]
$$

where $f=f\left(x^{\mu}, p^{\mu}\right)$ is the phase-space distribution function, which may depend on the spacetime position and four-momentum of the particles. Recall that the FLRW 


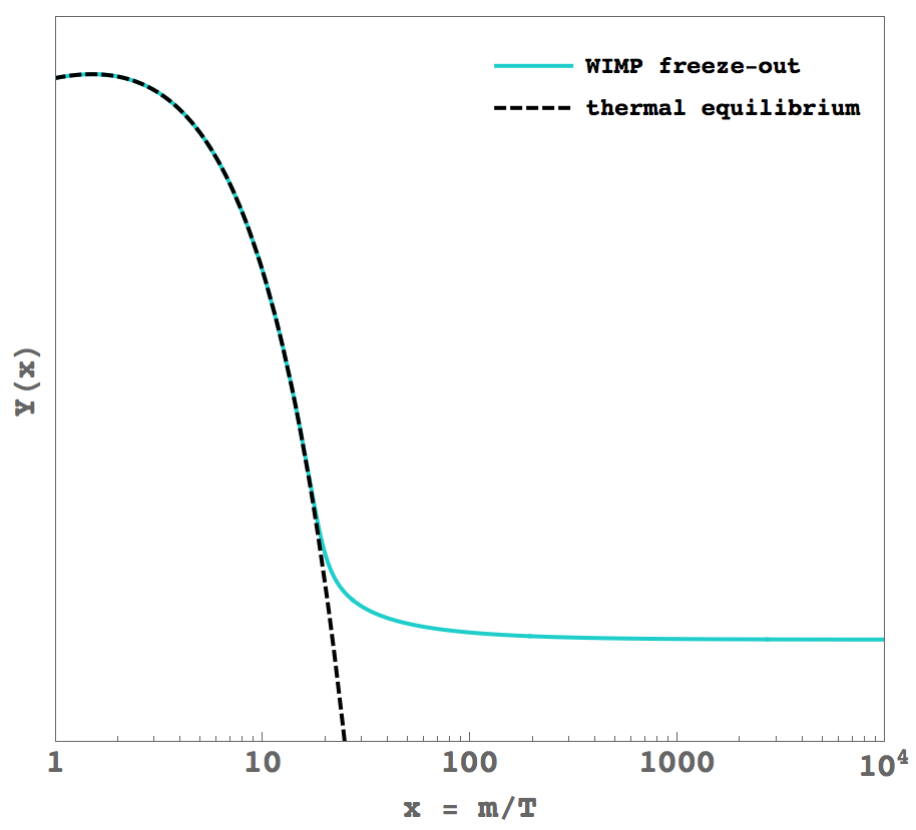

FiguRE 6.2. Freeze-out of a generic WIMP as a function of $x=m / T$. The dashed black line corresponds to a particle species that remains in thermal equilibrium to the present day. The solid light blue line corresponds to a WIMP particle that freezes out at $x \sim 20$. The vertical axis is the number density divided by the entropy density in arbitrary units on a log scale.

metric that we use to describe our Universe is homogenous and isotropic - that is, it is independent of position and direction. This reduces the parameter space of the phase space density function to only two degrees of freedom: energy and time. That 
is, $f\left(x^{\mu}, p^{\mu}\right) \rightarrow f(t, E)$. Then [17, 176],

$$
\begin{aligned}
\widehat{\mathbf{L}}[f(E, t)]=\frac{d f}{d \lambda} & =\frac{d x^{\mu}}{d \lambda} \frac{\partial f}{\partial x^{\mu}}+\frac{d p^{\mu}}{d \lambda} \frac{\partial f}{\partial p^{\mu}} \\
& =p^{\mu} \frac{\partial f}{\partial x^{\mu}}-\Gamma_{\alpha \beta}^{\mu} p^{\alpha} p^{\beta} \frac{\partial f}{\partial p^{\mu}} \\
& =E \frac{\partial f}{\partial t}-\Gamma_{\alpha \beta}^{0} p^{\alpha} p^{\beta} \frac{\partial f}{\partial E} \\
& =E \frac{\partial f}{\partial t}-\frac{\dot{a}}{a} h_{i j} p^{i} p^{j} \frac{\partial f}{\partial E} \\
& =E \frac{\partial f}{\partial t}-H|\vec{p}|^{2} \frac{\partial f}{\partial E}
\end{aligned}
$$

where we have used $x^{\mu}=(t, \vec{x}), p^{\mu}=(E, \vec{p})$, and $h_{i j}$ is the spatial part of the FLRW metriq ${ }^{1}$. This can be solved for $f(E, t)$ and substituted back into Equation (6.3). Integrating by parts gives

$$
\frac{d n}{d t}+3 H n=g \int \frac{d^{3} p}{(2 \pi)^{3} E} \widehat{\mathbf{C}}[f]
$$

In the case of scattering $12 \rightarrow 34$, the right-hand side becomes

$$
\begin{gathered}
-\sum_{\text {spin }} \int \frac{d^{3} p_{1}}{(2 \pi)^{3} E_{1}} \frac{d^{3} p_{2}}{(2 \pi)^{3} E_{2}} \frac{d^{3} p_{3}}{(2 \pi)^{3} E_{3}} \frac{d^{3} p_{4}}{(2 \pi)^{3} E_{4}}(2 \pi)^{4} \delta^{4}\left(p_{1}+p_{2}-p_{3}-p_{4}\right) \\
\times\left[f_{1}\left(\vec{p}_{1}\right) f_{2}\left(\vec{p}_{2}\right)\left(1 \pm f_{3}\left(\vec{p}_{3}\right)\right)\left(1 \pm f_{4}\left(\vec{p}_{4}\right)\right)\left|\mathcal{M}_{12 \rightarrow 34}\right|^{2}\right. \\
\left.\quad-f_{3}\left(\vec{p}_{3}\right) f_{4}\left(\vec{p}_{4}\right)\left(1 \pm f_{1}\left(\vec{p}_{1}\right)\right)\left(1 \pm f_{2}\left(\vec{p}_{2}\right)\right)\left|\mathcal{M}_{34 \rightarrow 12}\right|^{2}\right]
\end{gathered}
$$

where the \pm depends on whether the particle in question is a boson $(+)$ or a fermion $(-)$.

It is at this point that we make two assumptions. First, we assume invariance of $\mathcal{M}$ under time reversal during the freeze-out, so that $\left|\mathcal{M}_{34 \rightarrow 12}\right|^{2}=\left|\mathcal{M}_{12 \rightarrow 34}\right|^{2}=|\mathcal{M}|^{2}$. ${ }^{1}$ From the geodesic equation, $\frac{\partial p^{\mu}}{\partial \lambda}+\Gamma_{\alpha \beta}^{\mu} p^{\alpha} p^{\beta}=0$, where the Christoffel symbols (or affine connections), $\Gamma_{\beta \gamma}^{\alpha}$, are defined by partial derivatives of the metric tensor and are used to construct the Riemann tensor which describes the curvature at each point in a manifold [17. 
The second assumption is to use Maxwell-Boltzmann statistics rather than BoseEinstein or Fermi-Dirac, letting $1 \pm f \rightarrow 1$. This is a good approximation when $T \lesssim m$, as in the standard freeze-out process, so that the particles of interest are non-relativistic. Then Equation (6.9) becomes

$$
-\sum_{\text {spin }} \int \frac{d^{3} p_{3}}{(2 \pi)^{3} E_{3}} \frac{d^{3} p_{4}}{(2 \pi)^{3} E_{4}}(2 \pi)^{4} \delta^{4}\left(p_{1}+p_{2}-p_{3}-p_{4}\right)\left[f_{1}\left(\vec{p}_{1}\right) f_{2}\left(\vec{p}_{2}\right)-f_{3}\left(\vec{p}_{3}\right) f_{4}\left(\vec{p}_{4}\right)\right]|\mathcal{M}|^{2}
$$

which is simply the thermally-averaged cross section times the difference in individual number densities. If the particle is its own antiparticle, $n=n_{1}+n_{2}$, and

$$
\dot{n}+3 H n=-\langle\sigma \beta\rangle\left(n^{2}-n_{\mathrm{eq}}^{2}\right),
$$

where $\beta \equiv v_{\text {rel }} / c$. In terms of $Y \equiv n / s$,

$$
\dot{Y}=-s\langle\sigma \beta\rangle\left(Y^{2}-Y_{\mathrm{eq}}^{2}\right)
$$

To determine the relic density, we compare the present density of the $\mathrm{DM}^{2}$ (the present value of the number density divided by the entropy density, $Y_{0}$, should be the same as the value after freeze-out, $Y$ ) to the critical density (the density for a flat FLRW Universe),

$$
\Omega_{\mathrm{DM}} \equiv \frac{\rho_{0}}{\rho_{c}}=\frac{m s_{0} Y_{0}}{\frac{3}{8 \pi} H_{0}^{2} G} \propto \frac{1}{\langle\sigma \beta\rangle},
$$

where $s_{0}$ and $Y_{0}$ are the present-day values of entropy density and number density divided by entropy density, respectively. The value of the thermally-averaged cross section that corresponds to the observed DM density, $\Omega h^{2}=0.1188$, is roughly [183] given by

$$
\langle\sigma \beta\rangle_{\mathrm{STD}}=3 \times 10^{-26} \frac{\mathrm{cm}^{3}}{\mathrm{~s}} .
$$

\footnotetext{
${ }^{2}$ Notice $\rho_{0}=m n_{0} / a_{0}^{3}=m s_{0} n_{0} / s_{0} a_{0}^{3}=m s_{0} Y_{0} / a_{0}^{3}=m s_{0} Y_{0}$, since $a_{0}=a\left(t_{0}\right)=1$ by definition.
} 
In general, to perform the thermal average, we would convolve the cross section times velocity with a Boltzmann distribution of the velocities. In the non-relativistic limit, $\beta \ll 1$, we can expand the cross section times velocity in powers of $\beta, \sigma \beta=$ $a+b \beta^{2}+c \beta^{4}+\cdots$, to find

$$
\langle\sigma \beta\rangle=A+B\left\langle\beta^{2}\right\rangle+C\left\langle\beta^{4}\right\rangle+\cdots
$$

where $A=a, B=6 b / x$, and so on. In the simple case of s-wave annihilation (i.e., $a \neq 0$ rather than just $a \ll b)$, terms $B, C$, and higher can be neglected for $\beta \ll 1$, as is the case during freeze-out. In that case, to determine if our DM candidate can indeed be the DM observed in our Universe (or at least some fraction thereof), we compute the ratio

$$
\frac{\Omega_{X}}{\Omega_{\mathrm{DM}}}=\frac{\langle\sigma \beta\rangle_{\mathrm{STD}}}{\langle\sigma \beta(X X \rightarrow \mathrm{SM})\rangle},
$$

where $\langle\sigma \beta\rangle_{\mathrm{STD}}$ is given in Equation (6.15) and $\langle\sigma \beta(X X \rightarrow \mathrm{SM})\rangle$ is the cross section for the annihilation of our DM candidate to all relevant SM final states. We now describe how to perform this computation.

\subsection{Relic density of weak-scale large multiplet DM}

The $\zeta^{0, r}$ is a self-annihilating particle which interacts with the SM via gauge or Higgs boson exchange. As such, the final states for the annihilation of two $\zeta^{0, r}$ particles include $W^{+} W^{-}, Z Z, h h$, and $f \bar{f}$ (via $s$-channel Higgs exchange). The relevant Feynman diagrams are shown in Figure 6.3. We assume that all other members of the large multiplet, $\Sigma$, have decayed to $\zeta^{0, r}$ by this time, and will not affect the relic density. Thus, we neglect co-annihilations with other scalars from the large multiplet. We compute the annihilation cross sections in the zero-velocity limit. Because these 

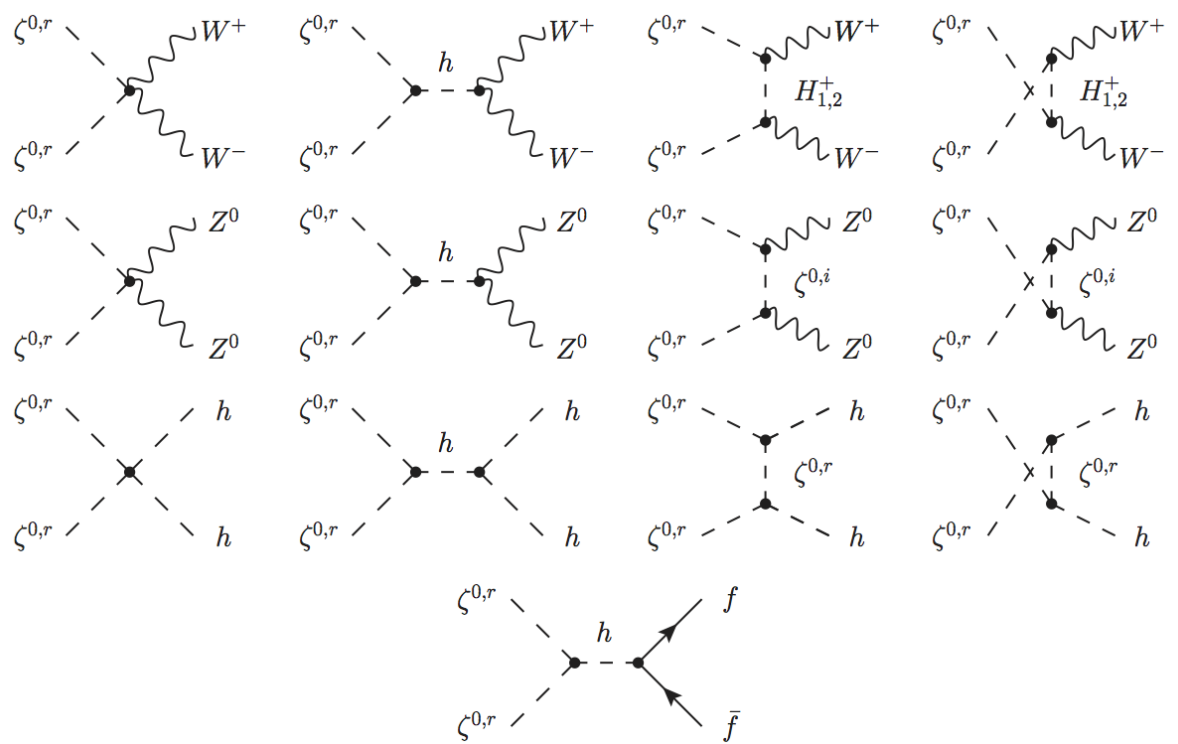

FiguRE 6.3. Feynman diagrams required for the computation of the relic abundance of $\zeta^{0, r}$.

cross sections are all nonzero in this limit, we do not need to average over the velocity distribution.

The annihilation cross sections to gauge boson two-body final states are given in the $\beta \rightarrow 0$ limit by,

$$
\begin{array}{r}
\sigma \beta\left(\zeta^{0, r} \zeta^{0, r} \rightarrow W^{+} W^{-}\right)=\frac{m_{W}^{4}}{8 \pi v} \sqrt{1-\frac{m_{W}^{2}}{m_{\zeta^{0, r}}^{2}}}\left[\frac{A_{W}^{2}}{m_{\zeta^{0, r}}^{2}}\left(3-4 \frac{m_{\zeta^{0, r}}^{2}}{m_{W}^{2}}+4 \frac{m_{\zeta^{0, r}}^{4}}{m_{W}^{4}}\right)\right. \\
+2 A_{W} B_{W}\left(1-3 \frac{m_{\zeta^{0, r}}^{2}}{m_{W}^{2}}+2 \frac{m_{\zeta^{0, r}}^{4}}{m_{W}^{4}}\right) \\
\left.+B_{W}^{2} m_{\zeta^{0, r}}^{2}\left(1-\frac{m_{\zeta^{0, r}}^{2}}{m_{W}^{2}}\right)^{2}\right]
\end{array}
$$


and

$$
\begin{array}{r}
\sigma \beta\left(\zeta^{0, r} \zeta^{0, r} \rightarrow Z Z\right)=\frac{m_{Z}^{4}}{16 \pi v} \sqrt{1-\frac{m_{Z}^{2}}{m_{\zeta^{0, r}}^{2}}}\left[\frac{A_{Z}^{2}}{m_{\zeta^{0, r}}^{2}}\left(3-4 \frac{m_{\zeta^{0, r}}^{2}}{m_{Z}^{2}}+4 \frac{m_{\zeta^{0, r}}^{4}}{m_{Z}^{4}}\right)\right. \\
+2 A_{Z} B_{Z}\left(1-3 \frac{m_{\zeta^{0, r}}^{2}}{m_{Z}^{2}}+2 \frac{m_{\zeta^{0, r}}^{4}}{m_{Z}^{4}}\right) \\
\left.+B_{Z}^{2} m_{\zeta^{0, r}}^{2}\left(1-\frac{m_{\zeta^{0, r}}^{2}}{m_{Z}^{2}}\right)^{2}\right]
\end{array}
$$

where the coefficients are given by

$$
\begin{aligned}
A_{Z} & =1+\frac{\Lambda_{n} v^{2}}{4 m_{\zeta^{0, r}}^{2}-M_{h}^{2}}, \\
B_{Z} & =\frac{4}{M_{Z}^{2}-m_{\zeta^{0, r}}^{2}-m_{\zeta^{0, i}}^{2}}, \\
A_{W} & =\frac{n^{2}-2}{2}+\frac{\Lambda_{n} v^{2}}{4 m_{\zeta^{0, r}}^{2}-M_{h}^{2}}, \\
B_{W} & =\frac{\left(n \cos \alpha_{1}-\sqrt{n^{2}-4} \sin \alpha_{1}\right)^{2}}{M_{W}^{2}-m_{\zeta^{0, r}}^{2}-m_{H_{1}^{+}}^{2}}+\frac{\left(-n \sin \alpha_{1}-\sqrt{n^{2}-4} \cos \alpha_{1}\right)^{2}}{M_{W}^{2}-m_{\zeta^{0, r}}^{2}-m_{H_{2}^{+}}^{2}}
\end{aligned}
$$

The mixing angles $\alpha_{Q}$ are given in Equation (3.11), defined by

$$
\begin{aligned}
\tan \alpha_{Q} & =(-1)^{\frac{n}{2}+Q+1} \frac{Q \lambda_{3}-\sqrt{Q^{2} \lambda_{3}^{2}+\left(n^{2}-4 Q^{2}\right) \lambda_{4}^{2}}}{\lambda_{4} \sqrt{n^{2}-4 Q^{2}}} \\
& =(-1)^{\frac{n}{2}+Q} \frac{\lambda_{4} \sqrt{n^{2}-4 Q^{2}}}{Q \lambda_{3}+\sqrt{Q^{2} \lambda_{3}^{2}+\left(n^{2}-4 Q^{2}\right) \lambda_{4}^{2}}} .
\end{aligned}
$$

The annihilation cross sections to Higgs and fermion final states are given by

$$
\sigma \beta\left(\zeta^{0, r} \zeta^{0, r} \rightarrow h h\right)=\frac{\Lambda_{n}^{2}}{64 \pi m_{\zeta^{0, r}}^{2}} \sqrt{1-\frac{m_{h}^{2}}{m_{\zeta^{0, r}}^{2}}}\left[1+\frac{3 m_{h}^{2}}{4 m_{\zeta^{0, r}}^{2}-m_{h}^{2}}-\frac{2 v \Lambda_{n}}{2 m_{\zeta^{0, r}}^{2}-m_{h}^{2}}\right]^{2},
$$


and

$$
\sigma \beta\left(\zeta^{0, r} \zeta^{0, r} \rightarrow f \bar{f}\right)=\frac{N_{c}}{4 \pi}\left[1-\frac{m_{f}^{2}}{m_{\zeta^{0, r}}^{2}}\right]^{\frac{3}{2}} \frac{m_{f}^{2} \Lambda_{n}^{2}}{\left(4 m_{\zeta^{0, r}}^{2}-m_{h}^{2}\right)^{2}},
$$

where $N_{c}$ is the number of colours of the final-state fermions, and recall that the Higgs coupling to $\zeta^{0, r} \zeta^{0, r}$ is given by

$$
\Lambda_{n}=\lambda_{2}+\frac{1}{4} \lambda_{3}+\frac{n}{2}(-1)^{n / 2+1} \lambda_{4}
$$

We note that when $m_{\zeta^{0, r}} \gg M_{W}, M_{Z}$, the annihilation cross sections for $\zeta^{0, r} \zeta^{0, r} \rightarrow$ $W^{+} W^{-}$and $Z Z$ go like $1 / m_{\zeta^{0, r}}^{2}$. In this limit, the new scalars become increasingly degenerate due to the unitarity constraints on the size of $\left|\lambda_{3}\right|$ and $\left|\lambda_{4}\right|$; the values of $A_{W, Z}$ and $B_{W, Z}$ are then related in such a way as to allow a cancellation of the $m_{\zeta^{0, r}}^{2} / M_{W, Z}^{2}$ and $m_{\zeta^{0, r}}^{4} / M_{W, Z}^{4}$ terms in the square brackets, which would otherwise make the cross section grow with increasing $m_{\zeta^{0, r}}$. This cancellation provides a nice cross-check of the matrix element calculation. We also checked our analytic results using CalcHEP [184].

We are now in a position to perform the calculation in Equation (6.17), where now

$$
\frac{\Omega_{\zeta}}{\Omega_{\mathrm{DM}}}=\frac{\langle\sigma \beta\rangle_{\mathrm{STD}}}{\left\langle\sigma \beta\left(\zeta^{0, r} \zeta^{0, r} \rightarrow W^{+} W^{-}, Z Z, h h, f \bar{f}\right)\right\rangle} .
$$

Simply scanning over all values of $m_{\zeta^{0, r}}, \lambda_{2}, \lambda_{3}$, and $\lambda_{4}$ would not be prudent, as we know from the previous chapters that many of these points fail to pass various theoretical and experimental constraints. In particular, we will make use of the unitarity and stability $\left(M^{2}>0\right)$ bounds from Chapter 4 , as well as the electroweak observables $(S T U)$ and Higgs decay $(h \rightarrow \gamma \gamma)$ bounds from Chapter 5 to reduce the number of calculations we perform. We generate a point in the $\left(m_{\zeta^{0, r}}, \lambda_{2}, \lambda_{3}, \lambda_{4}\right)$ space and check it against these four constraints. For each point that passes one 

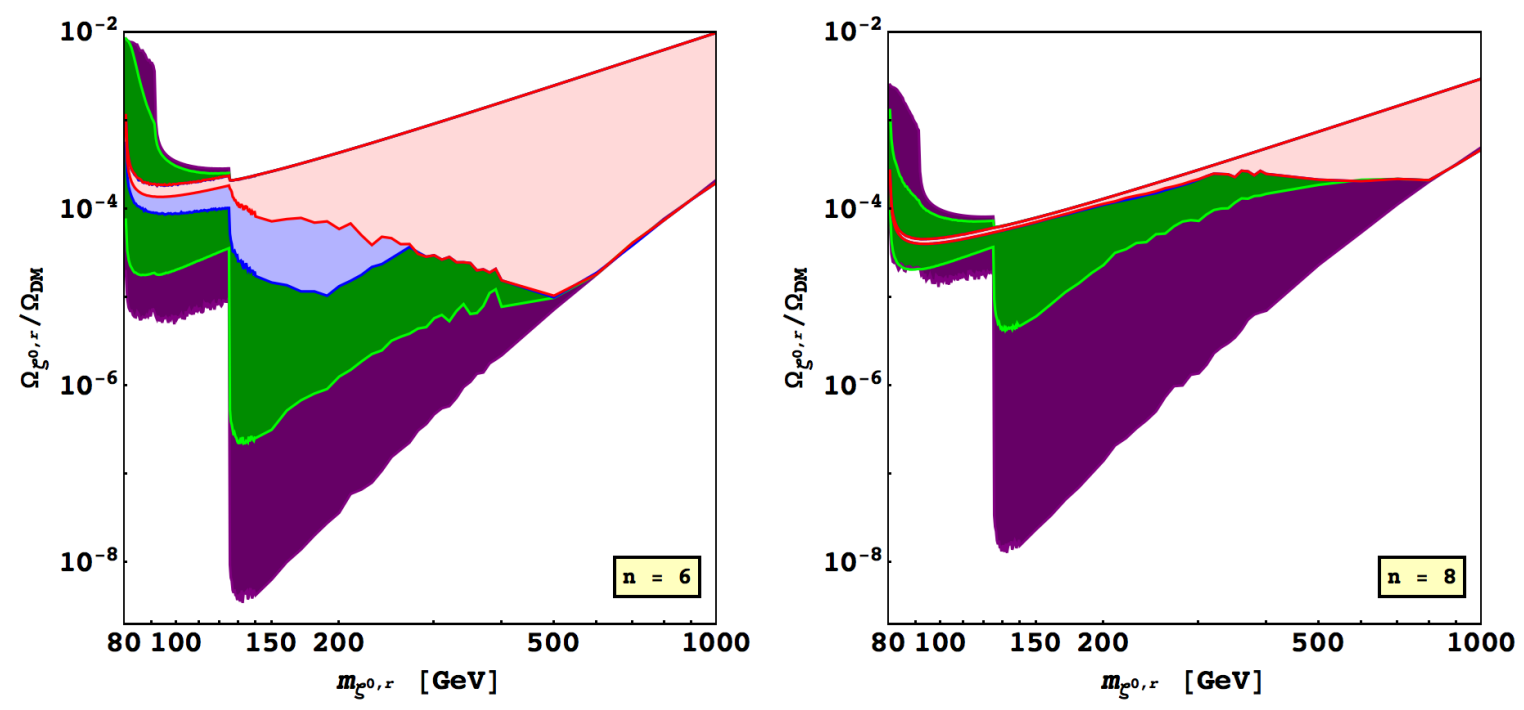

FiguRE 6.4. DM fraction for $\zeta^{0, r}$ as a function of $m_{\zeta^{0, r}}$. On the left is the DM fraction for $n=6$, and on the right is the DM fraction for $n=8$. The region allowed by all constraints in these plots is pink in both plots. $\zeta^{0, r}$ may make up only $\sim 0.01 \%$ of the total DM content over most of the weak-scale parameter space. The other regions are described in the text.

or more of the constraints, we calculate $\Omega_{\zeta} / \Omega_{\mathrm{DM}}$ and write it to a file. We then plot the regions that remain allowed after application of the constraints. The results are shown in Figure 6.4.

In these plots, we show the fraction of the total DM relic denisty which can be composed of $\zeta^{0, r}$. The region in pale pink is that which remains once all the constraints have been applied. We begin by scanning within the unitarity bounds on $\lambda_{2,3,4}$. This produces points in the dark purple, medium green, light blue, and pale pink regions. Any point in these regions falls within the unitarity bounds. Notice the quite obvious kinematic threshold for $\zeta^{0, r} \zeta^{0, r} \rightarrow h h$ at $m_{\zeta^{0, r}}=m_{h}$. Next, we use the bounds from the electroweak observables $S T U$. These points fall inside the medium green, light blue, and pale pink regions. Continuing on, we apply the bounds from $h \rightarrow \gamma \gamma$. This 
results in points in the light blue and pale pink regions. Finally, requiring $M^{2}>0$, we arrive at our fully constrained parameter space.

The final result of the relic density computation shows that, for masses below $1 \mathrm{TeV}, \zeta^{0, r}$ is certainly a DM candidate, but that there must be other sources of DM mass density. For the $n=6$ model, $\zeta^{0, r}$ may constitute at most $1 \%$ of the total DM content at $m_{\zeta^{0, r}}=1 \mathrm{TeV}$, while in the range $m_{W} \lesssim m_{\zeta^{0, r}} \lesssim 300 \mathrm{GeV}$, it may only constitute $\sim 0.01 \%$ of the total DM content. In the $n=8$ model, the situation is even more tightly constrained. At $m_{\zeta^{0, r}} \approx 1 \mathrm{TeV}, \zeta^{0, r}$ may only constitute $\sim 0.1 \%$ of the total DM content of the Universe, while in the range $m_{W} \lesssim m_{\zeta^{0, r}} \lesssim 200 \mathrm{GeV}$, it is less than $\sim 0.01 \%$.

We may wonder how the direct searches at the LHC Section 5.3) affect the parameter space. In those searches, we chose only four $\zeta^{0, r}$ mass values per model, and found constraints only on three of those mass values in $n=6$ and two in $n=8$. Because of this, it is difficult to translate these constraints onto Figure 6.4. We instead zoom-in on the appropriate mass range and perform the scan at those points. The results are shown in Figure 6.5. The points which are allowed by unitarity, STU, $h \rightarrow \gamma \gamma, M^{2}>0$, and the LHC direct search constraints are shown in pale green, while the pink region is those points which are allowed by only unitarity, $S T U, h \rightarrow \gamma \gamma$, and $M^{2}>0$ (as in Figure 6.4). Notice that, in the $n=8$ plot, there is essentially no additional constraint on the relic density arising from the LHC direct searches. In the $n=6$ plot, the relic density at $m_{\zeta^{0, r}} \geq 120 \mathrm{GeV}$ is similarly unaffected. Although the parameter space at $m_{\zeta^{0, r}}=80 \mathrm{GeV}$ is strongly constrained by the LHC direct search, the overall effect on the relic density is minimal. 

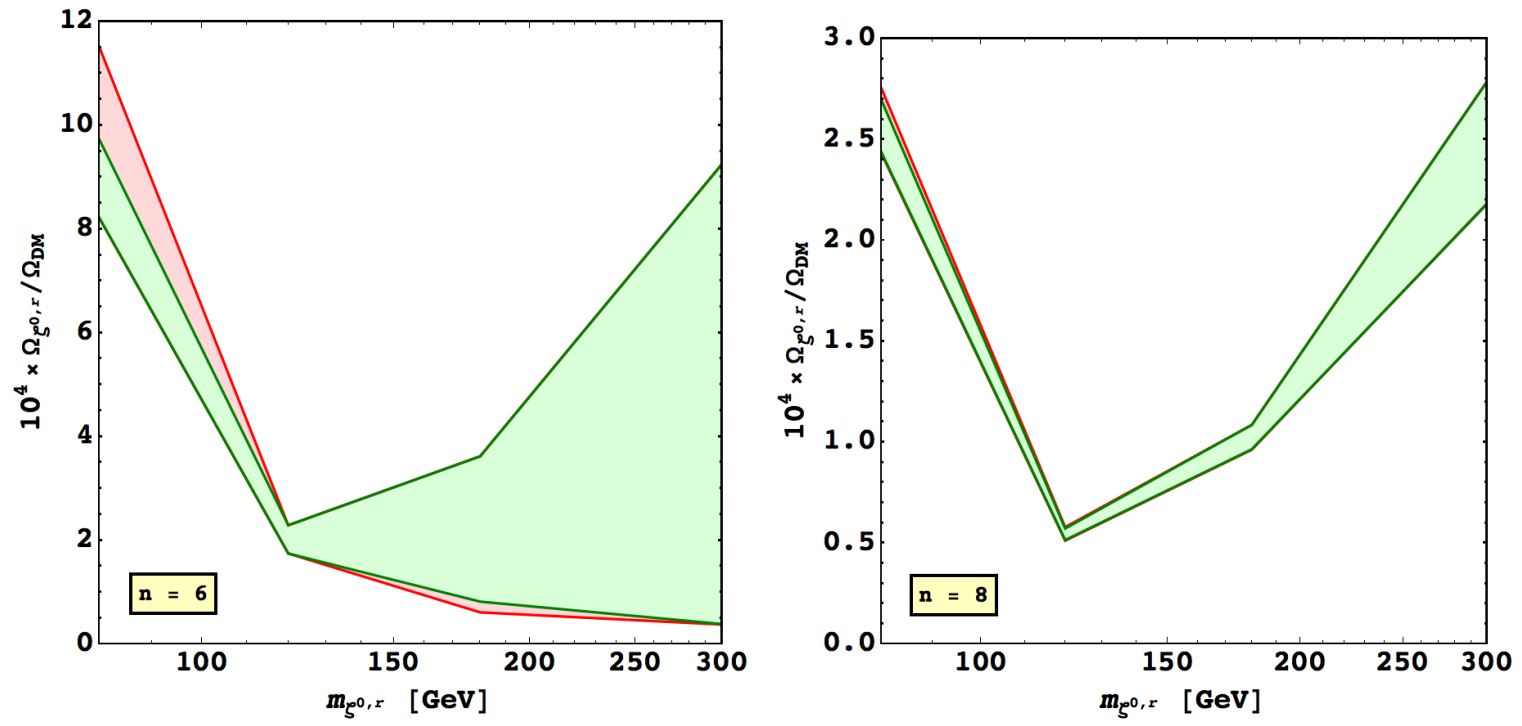

Figure 6.5. DM fraction for $\zeta^{0, r}$ as a function of $m_{\zeta^{0, r}}$. On the left is the DM fraction for $n=6$, and on the right is the DM fraction for $n=8$. The region allowed by all constraints in these plots is green in both plots, while the pink region is the same as in Figure 6.4.

\subsection{Relic density of multi-TeV large multiplet DM}

Based on the forms of the annihilation cross sections in Equations (6.18), (6.19), $(6.22)$, (6.23) and $(6.25)$, when $m_{\zeta^{0, r}} \gg m_{h}$,

$$
\frac{\Omega_{\zeta}}{\Omega_{\mathrm{DM}}} \propto m_{\zeta^{0, r}}^{2}\langle\sigma \beta\rangle_{\mathrm{STD}}
$$

From this, we expect there to be a DM mass where $\Omega_{\zeta} / \Omega_{\mathrm{DM}}=1$. From Equation (6.26), we would naïvely expect this to occur at $m_{\zeta^{0, r}} \approx 20 \mathrm{TeV}$. Since the only apparent constraint on the parameter space for $m_{\zeta^{0, r}}>1 \mathrm{TeV}$ appears to be from perturbative unitarity, we could simply apply this and solve for where $\Omega_{\zeta} / \Omega_{\mathrm{DM}}=1$. Indeed, we plot this in Figure 6.6. From these plots, we find that $\Omega_{\zeta} / \Omega_{\mathrm{DM}}=1$ corresponds to $10.1 \mathrm{TeV} \leq m_{\zeta^{0, r}} \leq 27.0 \mathrm{TeV}$ in the $n=6$ model and $18.4 \mathrm{TeV} \leq m_{\zeta^{0, r}} \leq 28.4 \mathrm{TeV}$ in the $n=8$ model. At this point, we could deduce the direct detection cross section, 

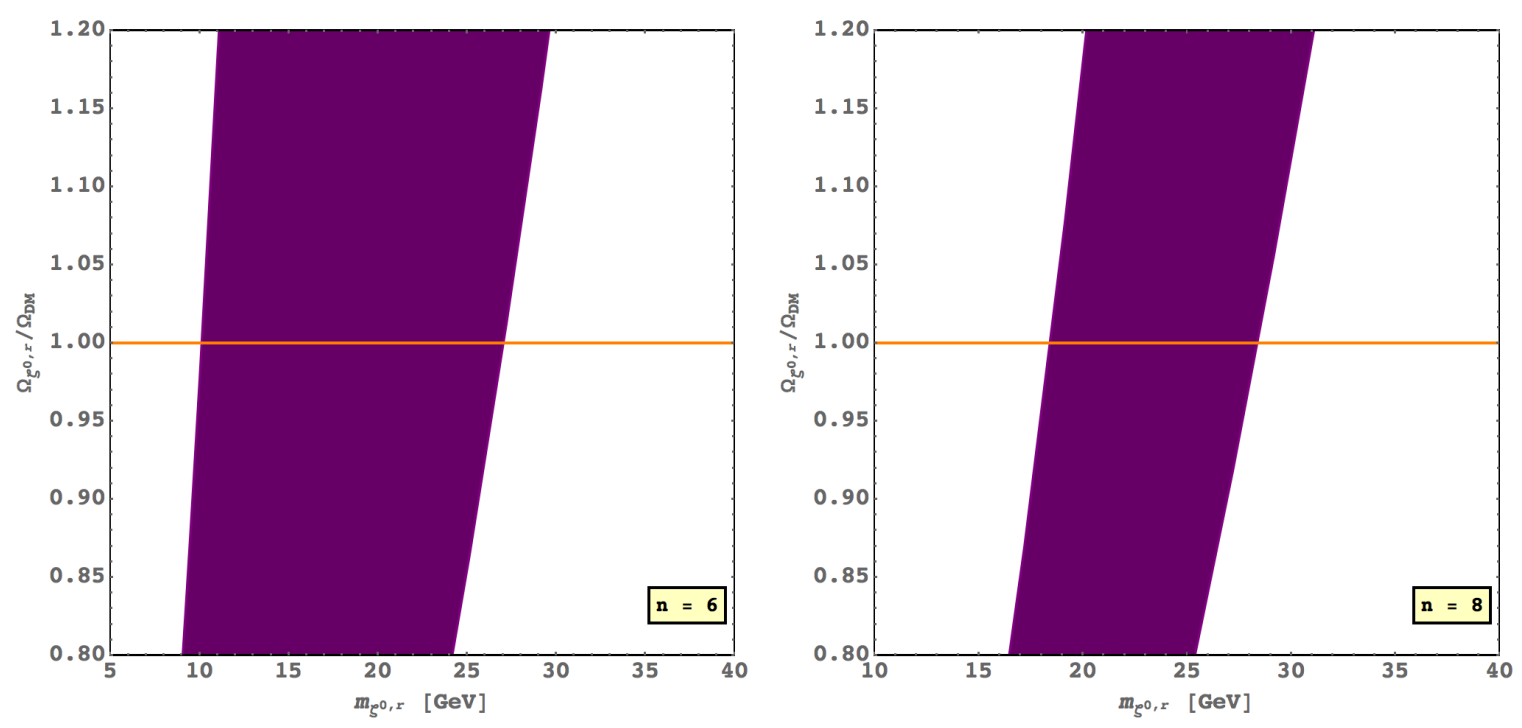

Figure 6.6. DM fraction as a function of $m_{\zeta^{0, r}}$ in the multi-TeV region, $n=6$ on the left and $n=8$ on the right. The allowed region in these plots is shaded in purple. The orange line corresponds to $\Omega_{\zeta} / \Omega_{\mathrm{DM}}=1$.

and end the story with those predictions. As the reader might expect, based on the remaining page count, this is not the whole of the story. There are two important effects for multi-TeV DM masses which did not need to be considered for weak-scale DM masses. The first is co-annihilation: when the heavier scalars in $\Sigma$ are close in mass to $\zeta^{0, r}$, they will be present in the thermal bath and affect the freeze-out calculation. The second is Sommerfeld enhancement: in the non-relativistic limit of particle annihilation or scattering, the perturbative approach breaks down and we must consider the effect of an effective long-range force between the interacting particles from the exchange of gauge and Higgs bosons.

\subsubsection{Co-annihilation.}

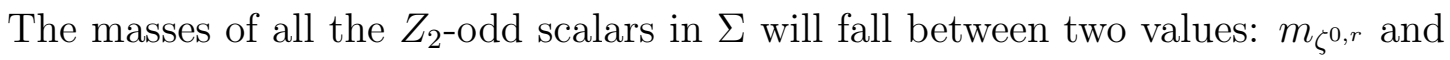
$m_{\zeta^{0, i}}$. The unitarity bounds, however, do not depend on the overall mass scale of $\Sigma$. 


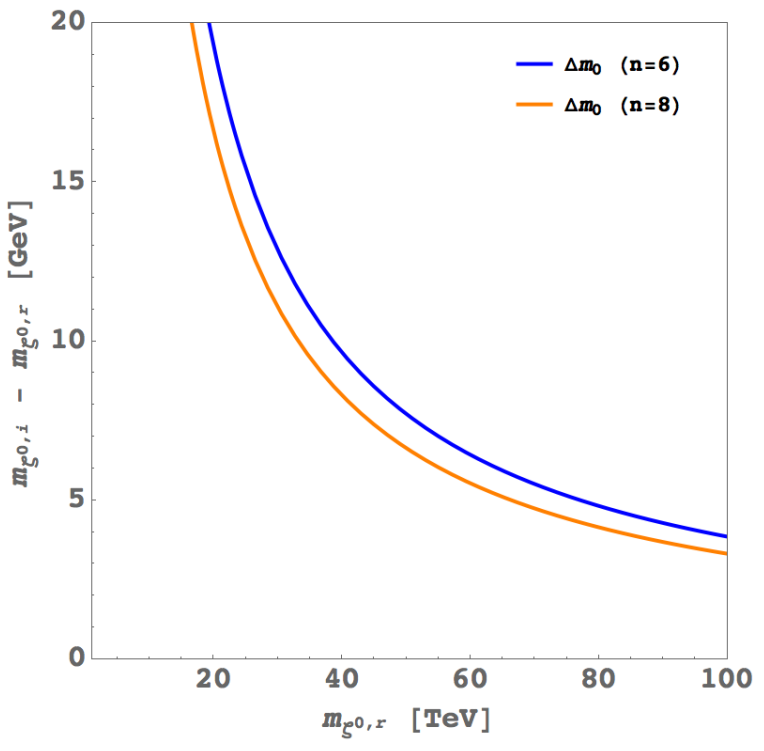

Figure 6.7. Mass splitting between $\zeta^{0, r}$ and $\zeta^{0, i}$ as a function of $m_{\zeta^{0, r}}$ in the multi-TeV region. The $n=6$ model is shown in blue and the $n=8$ model is shown in orange. As $m_{\zeta^{0, r}}$ increases, the overall mass splitting in the models will be very small. All of the charged states must have masses somewhere between the curve and the horizontal axis.

This means that the overall mass splitting will become squeezed as $m_{\zeta^{0, r}}$ increases, according to

$$
\begin{aligned}
\Delta m_{0} & =m_{\zeta^{0, i}}-m_{\zeta^{0, r}} \\
& =\sqrt{m_{\zeta^{0, r}}^{2}+\frac{n}{2}(-1)^{\frac{n}{2}} v^{2} \lambda_{4}}-m_{\zeta^{0, r}} \\
& \approx \frac{n}{4}\left|\lambda_{4}\right| \frac{v^{2}}{m_{\zeta^{0, r}}}+\mathcal{O}\left(\frac{v^{4}}{m_{\zeta^{0, r}}^{3}}\right) .
\end{aligned}
$$

Shown in Figure 6.7, we plot $\Delta m_{0}$ as a function of $m_{\zeta^{0, r}}$ for $n=6$ (solid blue) and $n=8$ (solid orange). For weak-scale DM as considered in Section 6.3, we made the assumption that all the heavier states of $\Sigma$ had decayed so that only $\zeta^{0, r}$ remained at freeze-out. When the mass splitting is strongly reduced, as in the multi-TeV DM case, the "heavier" states will still be present in the thermal bath. These co-annihilating 
states will affect the relic density of $\zeta^{0, r}$. In what follows, we make the approximation that all members of $\Sigma$ are degenerate as far as the equilibrium thermal density is concerned.

The DM fraction from Equation (6.17) becomes

$$
\frac{\Omega_{\zeta}}{\Omega_{\mathrm{DM}}}=\frac{\langle\sigma \beta\rangle_{\mathrm{STD}}}{\frac{1}{(2 n)^{2}} \sum_{Q}\left\langle\sigma \beta\left(\{\Sigma \Sigma\}^{Q} \rightarrow\left\{\mathrm{SM} \mathrm{SM}^{Q}\right)\right\rangle\right.},
$$

where the sum is over the appropriate charges, $Q=0, \pm 1, \pm 2$, and the factor of $1 /(2 n)^{2}$ in the denominator accounts for particles not meeting the "right" parter to annihilate in the early Universe. The required charge combinations from $\Sigma$ are, for $n=6$,

$$
\begin{aligned}
& \{\Sigma \Sigma\}^{0} \in\left\{\zeta^{0, r} \zeta^{0, r}, \zeta^{0, r} \zeta^{0, i}, \zeta^{0, i} \zeta^{0, r}, H_{k}^{+q} H_{\ell}^{-q}, \zeta^{+3} \zeta^{-3}\right\}, \\
& \{\Sigma \Sigma\}^{ \pm 1} \in\left\{\zeta^{0, r} H_{k}^{ \pm 1}, \zeta^{0, i} H_{k}^{ \pm 1}, H_{k}^{ \pm q} H_{\ell}^{\mp(q-1)}, \zeta^{ \pm 3} H_{k}^{\mp 2}\right\}, \\
& \{\Sigma \Sigma\}^{ \pm 2} \in\left\{\zeta^{0, r} H_{k}^{ \pm 2}, \zeta^{0, i} H_{k}^{ \pm 2}, H_{k}^{ \pm 1} H_{\ell}^{ \pm 1}, \zeta^{ \pm 3} H_{k}^{\mp 1}\right\},
\end{aligned}
$$

and for $n=8$,

$$
\begin{gathered}
\{\Sigma \Sigma\}^{0} \in\left\{\zeta^{0, r} \zeta^{0, r}, \zeta^{0, r} \zeta^{0, i}, \zeta^{0, i} \zeta^{0, r}, H_{k}^{+q} H_{\ell}^{-q}, \zeta^{+4} \zeta^{-4}\right\}, \\
\{\Sigma \Sigma\}^{ \pm 1} \in\left\{\zeta^{0, r} H_{k}^{ \pm 1}, \zeta^{0, i} H_{k}^{ \pm 1}, H_{k}^{ \pm q} H_{\ell}^{\mp(q-1)}, \zeta^{ \pm 4} H_{k}^{\mp 3}\right\}, \\
\{\Sigma \Sigma\}^{ \pm 2} \in\left\{\zeta^{0, r} H_{k}^{ \pm 2}, \zeta^{0, i} H_{k}^{ \pm 2}, H_{k}^{ \pm 1} H_{\ell}^{ \pm 1}, H_{k}^{ \pm 3} H_{\ell}^{\mp 1}, \zeta^{ \pm 4} H_{k}^{\mp 2}\right\},
\end{gathered}
$$

where $k, \ell=1,2$ and $q=1, \ldots, n / 2-1$, while the SM combinations are

$$
\begin{aligned}
&\left\{\mathrm{SM} \mathrm{SM}^{0} \in\left\{W^{+} W^{-}, Z Z, Z \gamma, \gamma \gamma, h h, h \gamma, h Z, f \bar{f}\right\}\right. \\
&\{\mathrm{SM} \mathrm{SM}\}^{ \pm 1} \in\left\{W^{ \pm} Z, W^{ \pm} \gamma, W^{ \pm} h, f f^{\prime}\right\}, \\
&\{\mathrm{SM} \mathrm{SM}\}^{ \pm 2} \in\left\{W^{ \pm} W^{ \pm}\right\},
\end{aligned}
$$




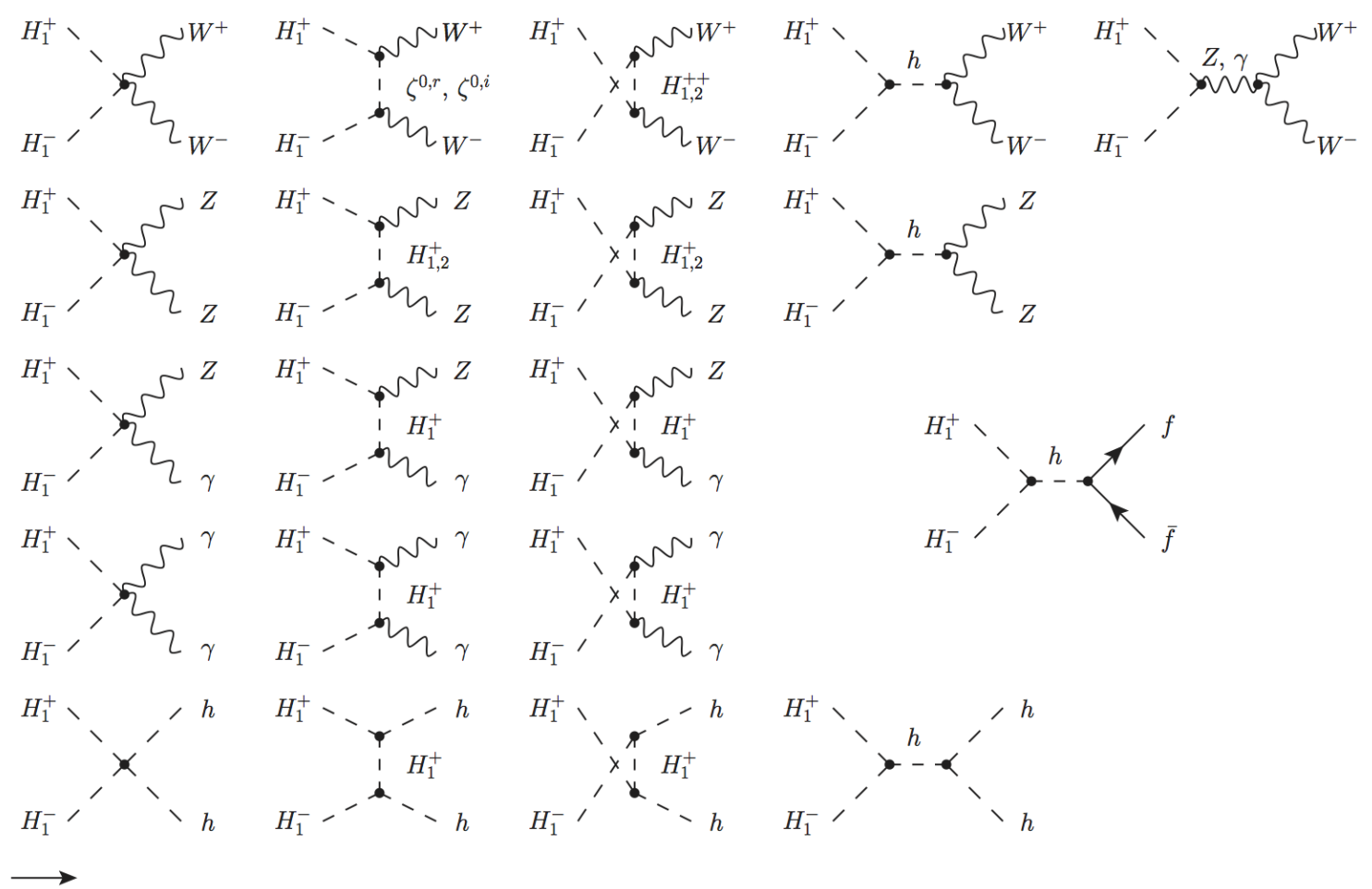

Figure 6.8. Feynman diagrams used in calculating the relic abundance of $H_{1}^{+1}$. The valid final states are $h h, W^{+} W^{-}, Z Z, Z \gamma, \gamma \gamma$, and $f \bar{f}$. The other states in the multiplet have similarly large numbers of diagrams.

Feynman diagrams for just the $H_{1}^{+} H_{1}^{-} \rightarrow\{\mathrm{SMSM}\}^{0}$ contribution are shown in Figure 6.8. Other annihilation processes will have similarly large numbers of diagrams.

Recall that we are interested here in the region of parameter space where $\Omega_{\zeta} / \Omega_{\mathrm{DM}}=$ 1. In the multi-TeV mass region, the effect of $\lambda_{2,3}$ on the relic density is small (less than a $10 \%$ effect). For this reason, we choose to scan only over $\Lambda_{n}$, the coupling of two $\Sigma$ fields to the Higgs boson, as given in Equation (6.24). We therefore set $\lambda_{2}=\lambda_{3}=0$ and $\lambda_{4}=(-1)^{\frac{n}{2}} \frac{2 \Lambda_{n}}{n}$. We plot the result in Figure 6.9. The dashed line shows where $\Omega_{\zeta} / \Omega_{\mathrm{DM}}=1$ for no co-annihilations, Equation (6.25), while the solid line corresponds to $\Omega_{\zeta} / \Omega_{\mathrm{DM}}=1$ for the co-annihilation case, Equation (6.28). The 

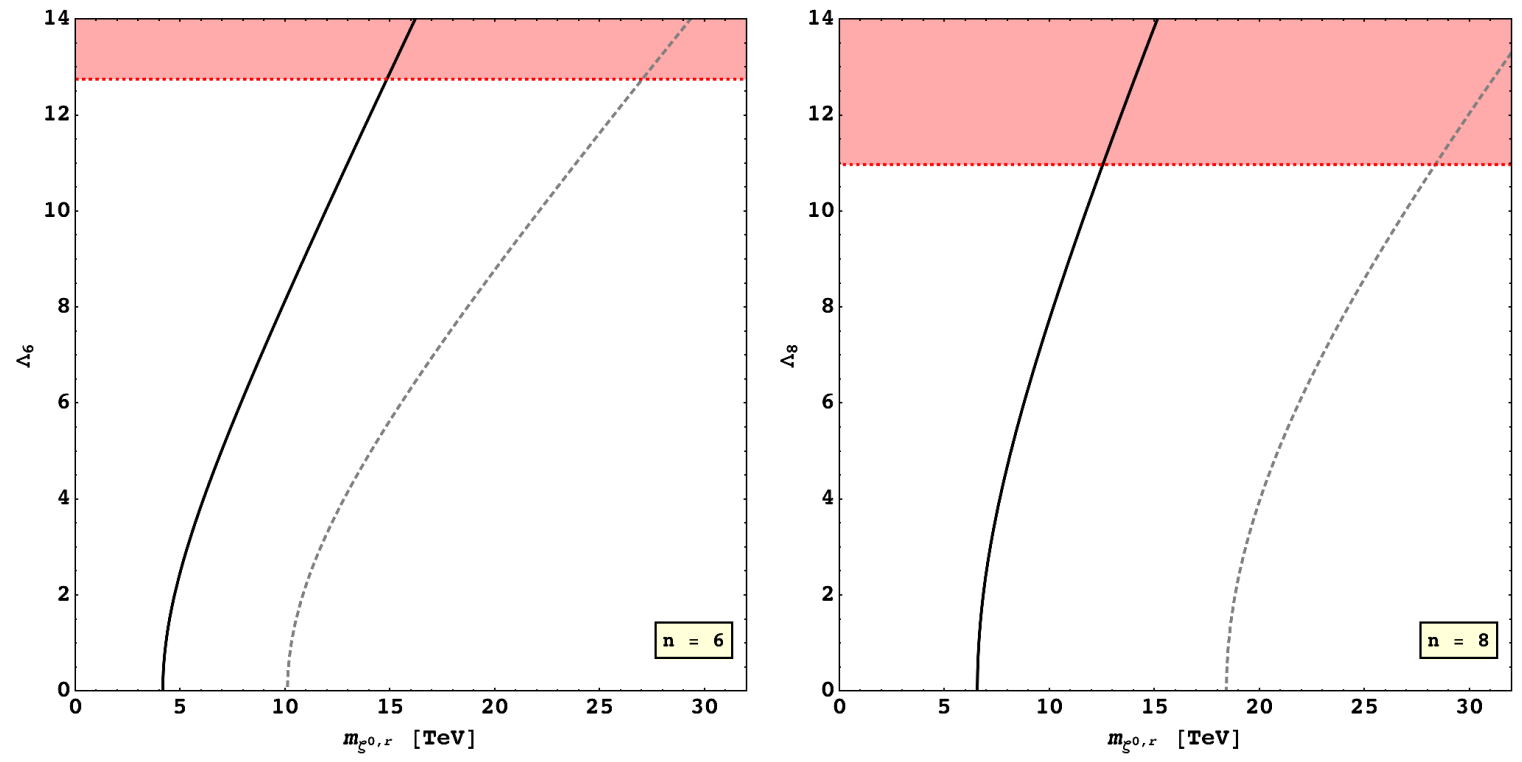

FIGURE 6.9. Plots showing where $\Omega_{\zeta} / \Omega_{\mathrm{DM}}=1$ incorporating coannihilation, with $n=6$ on the left and $n=8$ on the right. The solid line shows the curve where $\Omega_{\zeta} / \Omega_{\mathrm{DM}}=1$ including co-annihilations, and the dashed line shows where $\Omega_{\zeta} / \Omega_{\mathrm{DM}}=1$ without co-annihilations.

horizontal dashed red line gives the unitarity bound on $\Lambda_{n}^{\text {(unit.) }}=\frac{n}{2}\left|\lambda_{4}^{\text {(unit.) }}\right|$, where the numerical value of $\lambda_{4}^{\text {(unit.) }}$ is given in Table 4.2 .

When we compare the expressions for the DM fraction, Equations (6.25) and (6.28), we would expect that the denominator in the co-annihilation case would numerically be much larger than in the naïve single particle annihilation case - more contributions to the cross section will necessarily increase the total cross section, and decrease the DM fraction. This is the case as $\Lambda_{n} \rightarrow \Lambda_{n}^{\text {(unit.) }}$. However, being that there are more members of the multiplet present in the thermal bath, it is more likely that any two particles that meet will not be able to annihilate (e.g., there is no SM final state that could accommodate the situation where $\zeta^{0, r}$ meets $\left.\zeta^{+4}\right)$. Thus, we must average over the number of states in the multiplet by multiplying the sum in the denominator 
of Equation (6.28) by $1 /(2 n)^{2}$. This factor in the denominator will substantially decrease the total cross section over the entire mass range, increasing the DM fraction. In both cases, the fraction is pushed up so that the mass range required for $\Omega_{\zeta} / \Omega_{\mathrm{DM}}=1$ is lower, and also narrower (10.7 TeV and $6 \mathrm{TeV}$ rather than $16.9 \mathrm{TeV}$ and $10 \mathrm{TeV})$. In the case where co-annihilations are present, we find that $\Omega_{\zeta} / \Omega_{\mathrm{DM}}=1$ corresponds to $4.2 \mathrm{TeV} \leq m_{\zeta^{0, r}} \leq 14.9 \mathrm{TeV}$ in $n=6$ and $6.5 \mathrm{TeV} \leq m_{\zeta^{0, r}} \leq 12.5 \mathrm{TeV}$ in $n=8$.

\subsubsection{Sommerfeld enhancement.}

Consider a particle moving non-relativistically through space. The wave-function that describes the particle is a solution to the time-independent Schrödinger equation 185,

$$
-\frac{1}{2 m} \nabla^{2} \psi^{(0)}(\vec{r})=E \psi^{(0)}(\vec{r})
$$

For a particle moving along the positive $z$-axis, the solution will be

$$
\psi^{(0)}(\vec{r})=\mathrm{e}^{i k z}
$$

where $k \equiv \sqrt{2 m E}$. The amplitude at the origin will be $\left|\psi^{(0)}(0)\right|^{2}$.

If we now introduce a central potential, $V(r)$, which may be attractive or repulsive, then we will induce scattering and modify the wave function at the origin.

Rather than Equation (6.32), we have

$$
-\frac{1}{2 m} \nabla^{2} \psi(\vec{r})+V(r) \psi(\vec{r})=E \psi(\vec{r})
$$

In this case, there is no general solution for arbitrary $V(r)$. For $r$ such that $|E| \lesssim|V|$, the potential will distort the wave function. If we consider the case where we have plane waves coming in, which scatter from the potential, $V(r)$, and spherical waves 
are seen exiting at large $r$, then we have the asymptotic solution

$$
\psi(\vec{r}) \stackrel{r \rightarrow \infty}{\longrightarrow} \mathrm{e}^{i k z}+f(\theta) \frac{\mathrm{e}^{i k r}}{r}
$$

The amplitude at the origin will be $|\psi(0)|^{2}$. Because of the potential, the amplitude of the wave function at the origin may be larger or smaller than if the potential was not there.

More generally, the cross section for a short-distance annihilation process will be proportional to the square of the amplitude of the wave function at the origin. If we compare the cross section to its unperturbed value,

$$
\frac{\sigma}{\sigma_{0}}=\frac{|\psi(0)|^{2}}{\left|\psi^{(0)}(0)\right|^{2}} \equiv \mathcal{S}
$$

where $\mathcal{S}$ is the Sommerfeld factor [186]. We can then write a Sommerfeld-enhanced cross section as

$$
\sigma=\mathcal{S} \sigma_{0}
$$

Sommerfeld enhancement was first applied to the radiative corrections to pair annihilation for a Coulomb potential [187, 188]. Its importance for DM annihilation was first described in Ref. [189].

In particle physics, we examine annihilation processes through the perturbative process of Feynman diagrams. Sommerfeld enhancement is an inherently nonperturbative process that describes an infinite series of loops in a ladder diagram, as in the top half of Figure 6.10. The exchange of particles between the DM act as a long-range force, which affects the annihilation cross section as in Equation (6.37), Because it affects the cross section only as a multiplicative factor, we may factorize 


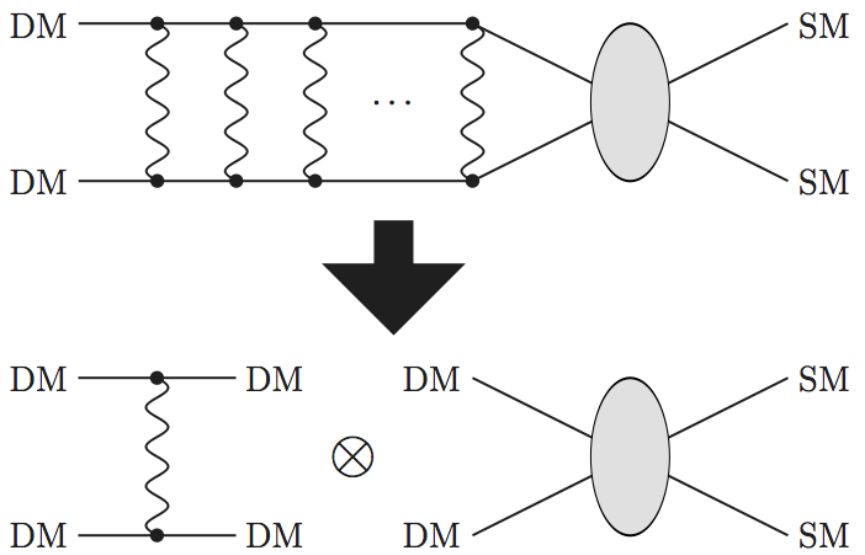

Figure 6.10. Feynman diagrams corresponding to Sommerfeld enhancement. In the top figure, the non-perturbative nature of Sommerfeld enhancement is manifest. The exchange of bosons act as an effective long-range force, increasing or decreasing the annihilation cross section. We can account for the Sommerfeld enhancement as a multiplicative factor (bottom diagram).

the calculation into a long-range (Sommerfeld) part and a short-range (annihilation) part (bottom half of Figure 6.10).

In the case of elementary particles we consider the two-particle wave-function, $\psi$, and the evolution of their long-range interaction is guided by the time-independent Schrödinger equation,

$$
-\frac{1}{2 \mathcal{M}} \frac{d^{2} \psi(r)}{d r^{2}}+V(r) \psi(r)=E \psi(r)
$$

where $\mathcal{M}$ is the reduced mass of the two-particle state, $E$ is its energy, and $V(r)$ is the potential caused by the interaction between the two particles prior to annihilation.

For Coulomb scattering,

$$
V_{\text {Coul. }}=\mp \frac{\alpha}{r},
$$


where - corresponds to an attractive potential, + corresponds to a repulsive potential, $\alpha$ is the coupling strength, and given the particle speed, $\beta$, we find [187]

$$
\mathcal{S}_{\text {Coul. }}=\eta \frac{2 \pi}{\epsilon_{\beta}} \frac{1}{1-\mathrm{e}^{-\eta \frac{2 \pi}{\epsilon_{\beta}}}},
$$

where $\epsilon_{\beta} \equiv \beta / \alpha$ and $\eta=+1(-1)$ for an attractive (repulsive) Coulomb potential. The effect is strongest when $\beta$ is small; formally, as $\beta \rightarrow \infty, \mathcal{S} \rightarrow 1$, there is no enhancement [190]. For small $\beta$, the attractive potential gives $\mathcal{S} \sim 2 \pi \alpha / \beta$, while the repulsive potential gives $\mathcal{S} \sim \mathrm{e}^{-2 \pi \alpha / \beta}$. We plot contours of constant $\mathcal{S}_{\text {Coul. }}(\beta, \alpha)$ in Figure 6.11. The left-hand plot shows the attractive case, $V=-\alpha / r$, and the right-hand plot shows the repulsive case, $V=+\alpha / r$. In both cases, $\alpha>0$. In the attractive case, the Sommerfeld factor is greater than one, and quickly increases as $\beta \rightarrow 0$. In the repulsive case, the inverse effect occurs: as $\beta \rightarrow 0$, the Sommerfeld factor decreases quickly to 0 .

For the case of a Yukawa-like potential,

$$
V_{\text {Yuk. }}= \pm \frac{\alpha}{r} \mathrm{e}^{-m r}
$$

where, as in the Coulomb case, - corresponds to an attractive potential, + corresponds to a repulsive potential, $\alpha$ is the coupling strength, and now $m$ is the mass of the exchanged particle. Unfortunately, this does not have a nice, analytic solution like $V_{\text {Coul. }}$, and we would need to determine $\mathcal{S}$ numerically. Fortunately, though, there is a similar potential, the Hulthén potential [191, 192], which exhibits the same behaviour in both the small- $r$ and large- $r$ limits. It was shown in Ref. [45] that the Hulthén potential reproduces the numerical results for the Yukawa potential to better than 10\%, and accurately describe the resonant behaviour which will be 

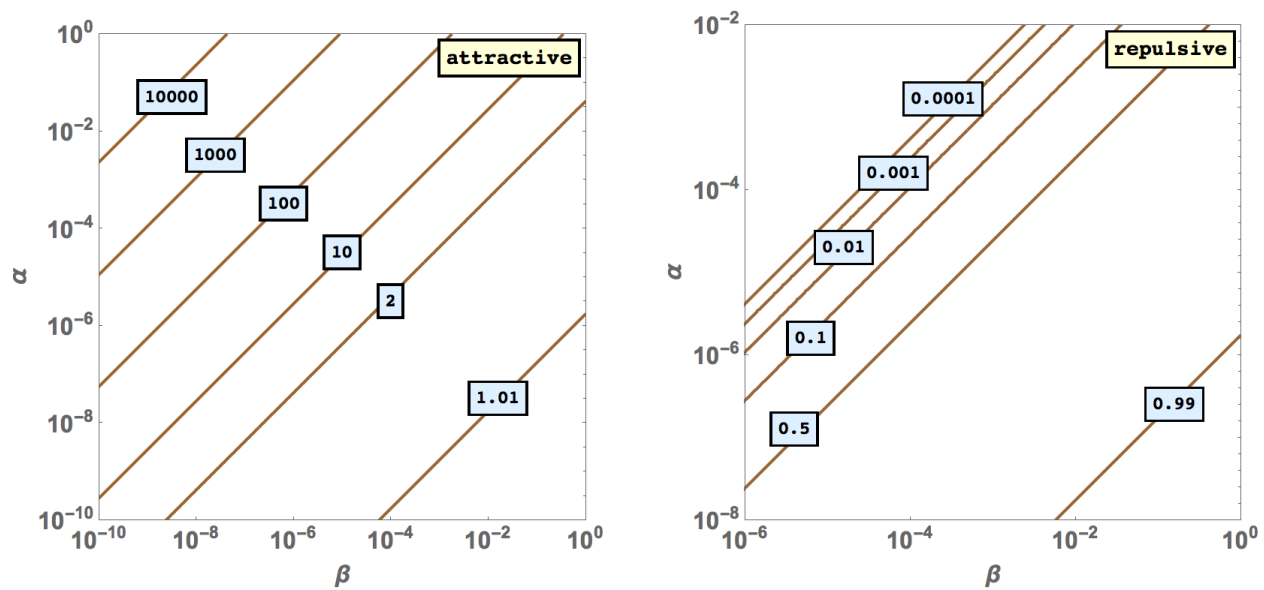

FigurE 6.11. Sommerfeld enhancement with a Coulomb potential. The left-hand plot shows contours of constant $\mathcal{S}_{\text {Coul. }}$ for $V=-\alpha / r$ (attractive), while the right-hand plot shows contours of constant $\mathcal{S}_{\text {Coul. }}$ for $V=+\alpha / r$ (repulsive). Notice that the enhancement factor is greater than one in the attractive case, meaning the cross section is larger than it would be with no potential present, and less than one in the repulsive case, meaning the cross section is smaller than it would be with no potential present.

described below. More importantly, the time-independent Schrödinger equation with the Hulthén potential does have an analytic solution. The Hulthén potential is given by

$$
V_{\mathrm{Hul} .}= \pm \alpha \omega \frac{\mathrm{e}^{-\omega r}}{1-\mathrm{e}^{-\omega r}}
$$

where $m=\pi^{2} \omega / 6$. The Sommerfeld enhancement factor for the Hulthén potential is given by [193] (see also Ref. [194])

$$
\mathcal{S}=\frac{\pi}{\epsilon_{\beta}} \frac{\sinh \left(\frac{2 \pi \epsilon_{\beta}}{\epsilon_{\omega}}\right)}{\cosh \left(\frac{2 \pi \epsilon_{\beta}}{\epsilon_{\omega}}\right)-\cos \left(2 \pi \sqrt{\frac{1}{\epsilon_{\omega}}-\frac{\epsilon_{\beta}^{2}}{\epsilon_{\omega}^{2}}}\right)}
$$

where $\epsilon_{\beta} \equiv \beta / \alpha, \epsilon_{\omega} \equiv \omega / \alpha m_{\mathrm{DM}}$, and $m_{\mathrm{DM}}$ is the mass of the scattering particles. We plot the Sommerfeld enhancement factor for the Hulthén potential in Figure 6.12, 

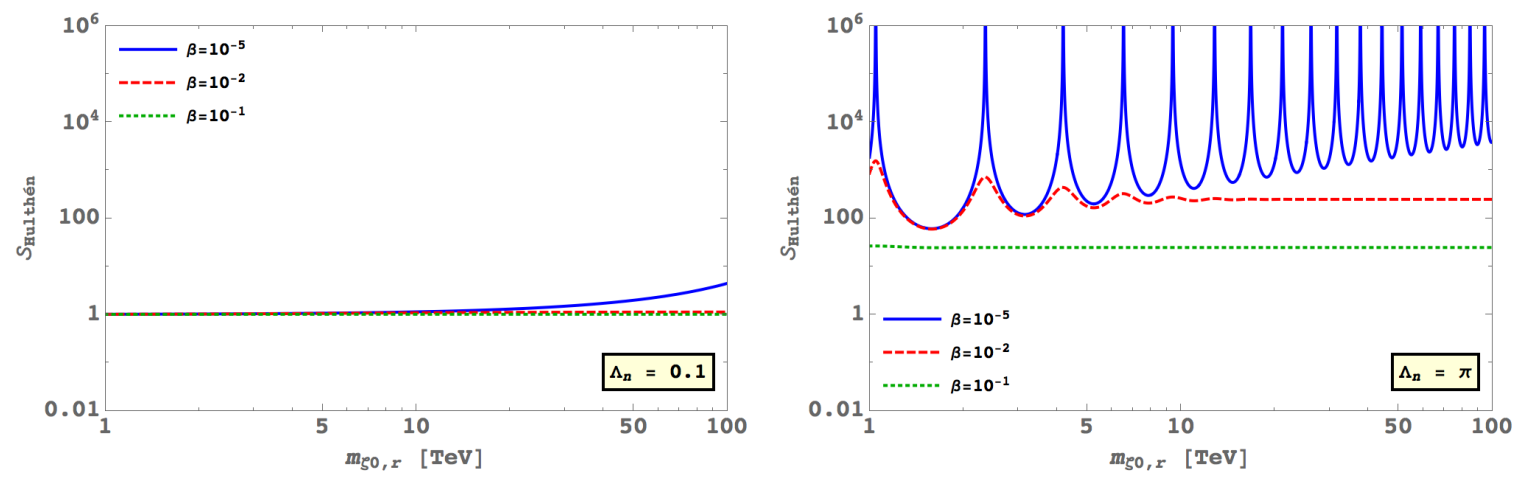

FiguRE 6.12. Sommerfeld enhancement with a Hulthén potential for $\Lambda_{n}=0.1$ (left plot) and $\Lambda_{n}=\pi$ (right plot). The curves correspond to different values of $\beta=v / c$. The solid blue curve corresponds to $\beta=10^{-5}$, while the dashed red to $\beta=10^{-2}$, and the dotted green to $\beta=10^{-1}$.

where $\omega=6 m_{h} / \pi^{2}, m_{\mathrm{DM}}=m_{\zeta^{0, r}}$, and $\alpha=\Lambda_{n}^{2} / 4 \pi$ (where these values come from will be shown just after the discussion of the features of the plots). Notice the structure present in these plots that is not present in the Coulomb case. As $\beta \rightarrow 0$, the energy of the incident particles is near zero. The number of bound states in the Coulomb case is infinite, and so changing the parameters of the potential (in this case, $\alpha$ ) will have no effect on the number of bound states. On the other hand, in the Yukawa (or Hulthén) potential, there are a finite number of bound states. If the potential is modified (in this case, either by changing $\epsilon_{\beta}$ or $\epsilon_{\omega}$ ), the number of bound states may change. That is, changes in these parameters will bring bound states nearer to $E=0$. If the energy of the incident particle (e.g., $E=+\delta$ for some small $\delta$ ) is close to a bound state $(E=-\delta)$, then the interaction will be resonantly enhanced, leading to the spikes in the right-hand plot of Figure 6.12, where the coupling is large and the velocity is small. 
The calculation of the potential, $V(r)$, is done using the Born approximation, (see, e.g., Chapter 4 of Ref. [112]), where

$$
V(r)=\int \frac{d^{3} q}{(2 \pi)^{3}} \tilde{V}(\vec{q}) e^{i \vec{q} \cdot \vec{r}},
$$

where $\tilde{V}(\vec{q}) \approx-\mathcal{M}$ and $q^{2}=\left(p_{2}-p_{3}\right)^{2} \approx-\left|\vec{p}_{2}-\vec{p}_{3}\right|^{2}=-|\vec{q}|^{2}$. The scattering will proceed via the exchange of $\gamma, W^{ \pm}, Z$, or $h$. The matrix element for each is given below, where the couplings $C_{i j k}$ are given in Appendix $\mathrm{A}$, and $t$ and $u$ are the Mandelstam variables:

$$
\begin{aligned}
\mathcal{M}_{\gamma} & =-e^{2} C_{s_{1} s_{1} \gamma} C_{s_{2} s_{2} \gamma} \frac{t+u}{2 q^{2}} \\
\mathcal{M}_{W} & =e^{2} C_{s_{1} s_{4} W} C_{s_{2} s_{3} W}^{*} \frac{1}{q^{2}-m_{W}^{2}}\left(\frac{t^{2}}{m_{W}^{2}}-\frac{t+u}{2}\right), \\
\mathcal{M}_{Z} & =e^{2} C_{s_{1} s_{4} Z} C_{s_{2} s_{3} Z}^{*} \frac{1}{q^{2}-m_{Z}^{2}}\left(\frac{t^{2}}{m_{Z}^{2}}-\frac{t+u}{2}\right) \\
\mathcal{M}_{h} & =-C_{s_{1} s_{1} h} C_{s_{2} s_{2} h} \frac{1}{q^{2}-m_{h}^{2}}
\end{aligned}
$$

The corresponding potentials are then

$$
\begin{aligned}
& V_{\gamma}(r)=-\left[\alpha_{\mathrm{EM}} C_{s_{1} s_{1} \gamma} C_{s_{2} s_{2} \gamma}\right] \frac{1}{r} \quad=-g_{\gamma} \frac{1}{r}, \\
& V_{W}(r)=-\left[\alpha_{\mathrm{EM}} C_{s_{1} s_{4} W} C_{s_{2} s_{3} W}^{*}\right] \frac{\mathrm{e}^{-m_{W} r}}{r}=-g_{W} \frac{\mathrm{e}^{-m_{W} r}}{r}, \\
& V_{Z}(r)=-\left[\alpha_{\mathrm{EM}} C_{s_{1} s_{4} Z} C_{s_{2} s_{3} Z}^{*}\right] \frac{\mathrm{e}^{-m_{Z} r}}{r}=-g_{Z} \frac{\mathrm{e}^{-m_{Z} r}}{r}, \\
& V_{h}(r)=-\left[\frac{C_{s_{1} s_{1} h} C_{s_{2} s_{2} h}}{4 \pi}\right] \frac{\mathrm{e}^{-m_{h} r}}{r} \quad=-g_{h} \frac{\mathrm{e}^{-m_{h} r}}{r},
\end{aligned}
$$

where we define $g_{\gamma, W, Z, h}$ as the couplings in the square brackets, and the kinematic factors are suppressed because we are working in the low-energy limit. Notice on the 
far right-hand side, each of the last three may be put into a Yukawa-like form, and so we may use the Hulthén approximation.

In the case of $\zeta^{0, r} \zeta^{0, r} \rightarrow \mathrm{SMSM}$, the only relevant potential is $V_{h}(r)$, as the others are all zero. At temperatures above the electroweak phase transition, which would normally be the case in the multi-TeV mass region, the VEV would be zero. In that case, the $\zeta^{0, r} \zeta^{0, r} h$ coupling would also be zero, and there would be no enhancement. However, we would like to examine the form of the effects of Sommerfeld enhancement in this simpler case so we retain $v \neq 0$ for now. Then the Sommerfeld enhancement factor is given by Equation (6.43),

$$
\mathcal{S}_{h}=\frac{\pi}{\epsilon_{\beta}} \frac{\sinh \left(\frac{2 \pi \epsilon_{\beta}}{\epsilon_{\omega}}\right)}{\cosh \left(\frac{2 \pi \epsilon_{\beta}}{\epsilon_{\omega}}\right)-\cos \left(2 \pi \sqrt{\frac{1}{\epsilon_{\omega}}-\frac{\epsilon_{\beta}^{2}}{\epsilon_{\omega}^{2}}}\right)},
$$

where

$$
\epsilon_{\beta}=\frac{\beta}{\alpha_{\zeta}}, \quad \epsilon_{\omega}=\frac{\pi^{2}}{6} \frac{m_{h}}{\alpha_{\zeta} m_{\zeta^{0, r}}}, \quad \text { and } \quad \alpha_{\zeta}=\frac{\Lambda_{n}^{2}}{4 \pi} .
$$

Returning to the annihilation cross section, Equation (6.37), we will have $\sigma \beta=$ $\mathcal{S}_{h} \cdot \sigma_{0} \beta$, where $\sigma_{0} \beta$ is the same sum of the cross section times velocity as in Equation (6.25), Notice that $\mathcal{S}_{h}$ is a function of $\beta$, and so we will have to thermally-average its contribution,

$$
\langle\sigma \beta\rangle=\frac{x^{\frac{3}{2}}}{4 \pi} \int \mathcal{S}_{h} \sigma_{0} \beta \beta^{2} \mathrm{e}^{-\frac{x \beta^{2}}{4}} d \beta .
$$

As before, in the case of s-wave annihilation, the cross section times velocity is not a function of $\beta$, so it may be taken outside of the integral, and we are left with

$$
\langle\sigma \beta\rangle=\sigma_{0} \beta\left\langle\mathcal{S}_{h}(x)\right\rangle
$$




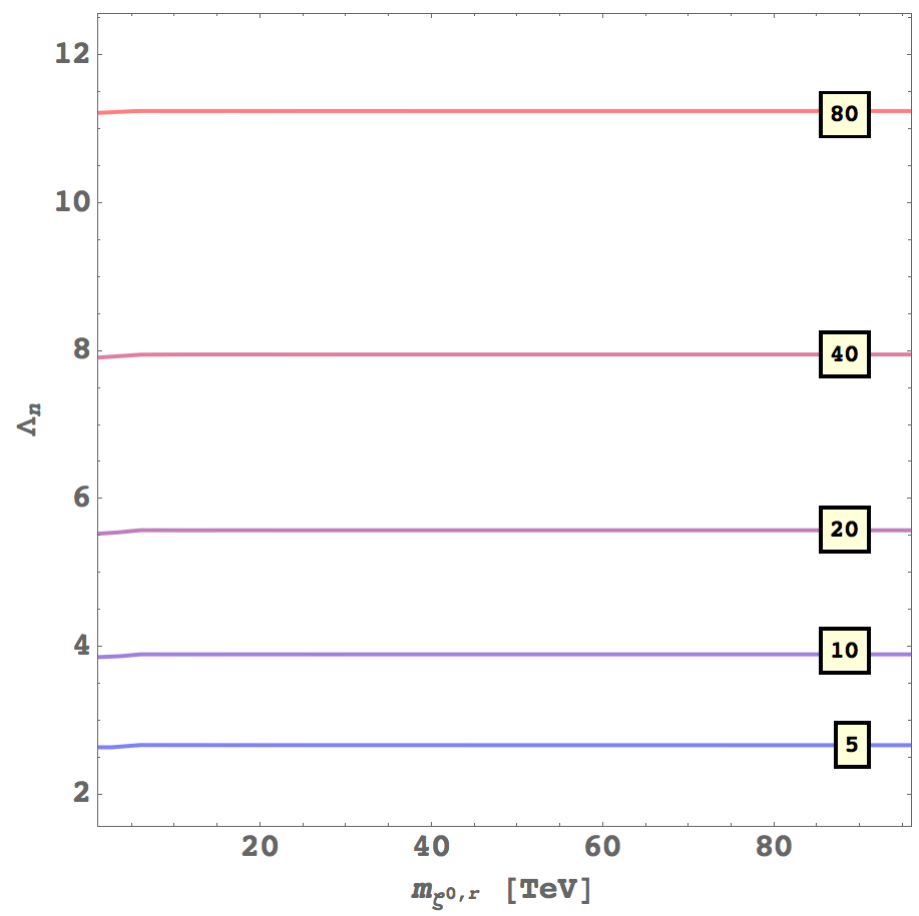

FiguRE 6.13. Thermally-averaged Sommerfeld enhancement with a Hulthén potential as a function of $m_{\zeta^{0, r}}$ and $\Lambda_{n}$. Contours are labelled with the value of $\mathcal{S}_{h}$. The thermally-averaged enhancement varies with $\Lambda_{n}$, but not with $m_{\zeta^{0, r}}$.

where

$$
\left\langle\mathcal{S}_{h}(x)\right\rangle \equiv \frac{x^{\frac{3}{2}}}{4 \pi} \int \mathcal{S}_{h} \beta^{2} \mathrm{e}^{-\frac{x \beta^{2}}{4}} d \beta .
$$

The effect of Sommerfeld enhancement in the early Universe is small, due to the high$\beta$ tail of the velocity distribution - recall that Sommerfeld enhancement is strongest when $\beta$ is small. We plot $\left\langle\mathcal{S}_{h}(x)\right\rangle$ as a function of $m_{\zeta^{0, r}}$ and $\Lambda_{n}$ (for the standard freeze-out value of $x=20$ ) in Figure 6.13 . The contours are labelled with the value of $\left\langle\mathcal{S}_{h}(x)\right\rangle$. The thermally-averaged Sommerfeld enhancement factor varies with $\Lambda_{n}$, but not with $m_{\zeta^{0, r}}$. Compare this to Figure 6.12 , where $\mathcal{S}_{h}$ was not thermally-averaged and exhibits resonance behaviour. 

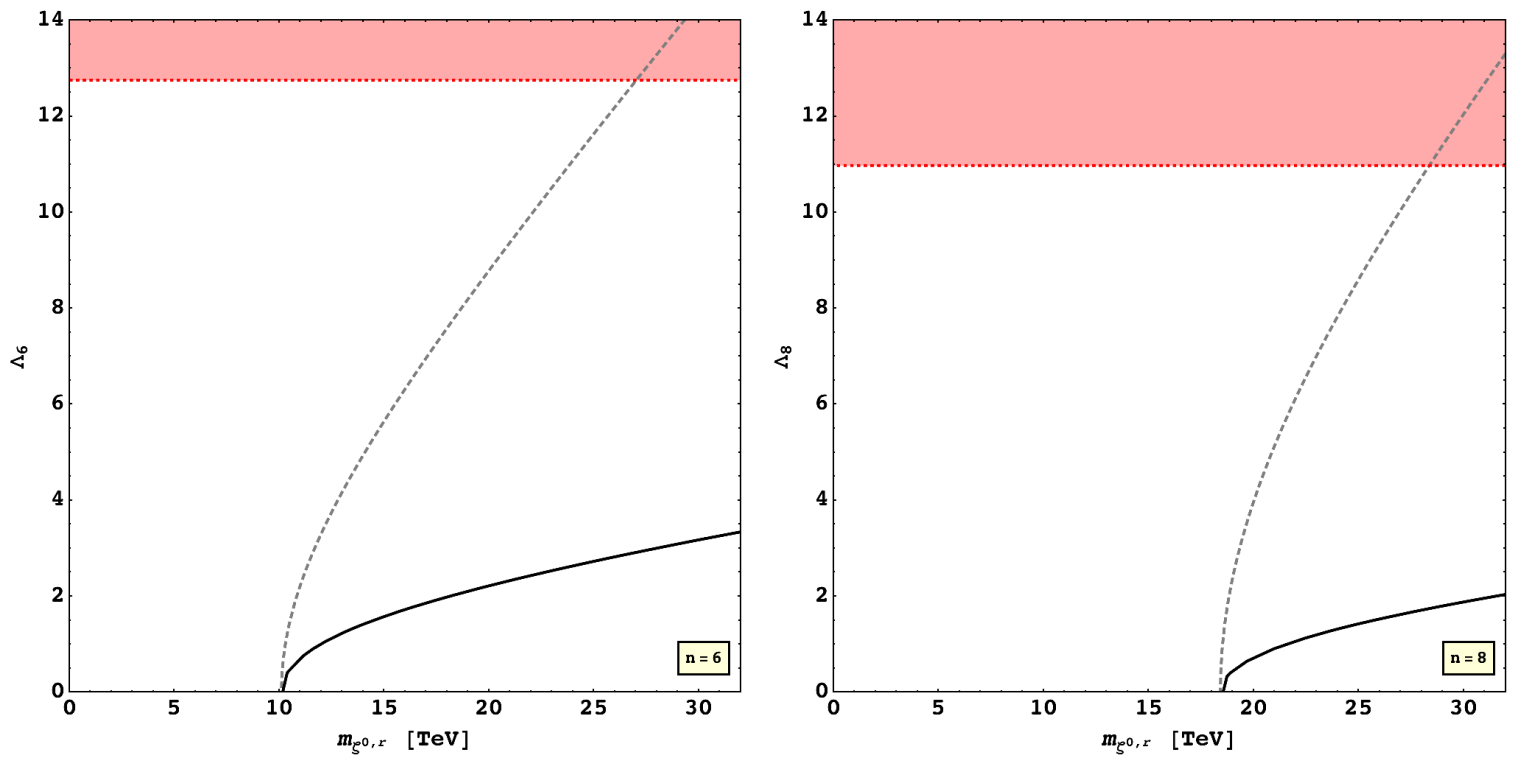

FIGURE 6.14. Plots showing where $\Omega_{\zeta} / \Omega_{\mathrm{DM}}=1$ incorporating Sommerfeld enhancement, with $n=6$ on the left and $n=8$ on the right. The solid line shows the curve where $\Omega_{\zeta} / \Omega_{\mathrm{DM}}=1$ including Sommerfeld enhancement with a Higgs potential, and the dashed line shows where $\Omega_{\zeta} / \Omega_{\mathrm{DM}}=1$ without Sommerfeld enhancement.

We now calculate the effect of Sommerfeld enhancement on the relic abundance of $\zeta^{0, r}$. We have seen that we can factor out the cross section from the thermal averaging, and so the DM fraction, Equation (6.17), will be

$$
\frac{\Omega_{\zeta}}{\Omega_{\mathrm{DM}}}=\frac{\langle\sigma \beta\rangle_{\mathrm{STD}}}{\left\langle\sigma \beta\left(\zeta^{0, r} \zeta^{0, r} \rightarrow W^{+} W^{-}, Z Z, h h, f \bar{f}\right)\right\rangle\left\langle\mathcal{S}_{h}(x)\right\rangle},
$$

where, again, we use $x=20$ and $\langle\sigma \beta\rangle_{\mathrm{STD}}=3 \times 10^{-26} \frac{\mathrm{cm}^{3}}{\mathrm{~s}}$. We plot the result in Figure 6.14. The dashed line shows where $\Omega_{\zeta} / \Omega_{\mathrm{DM}}=1$ for Equation (6.25), while the solid line corresponds to $\Omega_{\zeta} / \Omega_{\mathrm{DM}}=1$ for the Sommerfeld-enhanced case, Equation (6.52) The horizontal red line gives the unitarity bound on $\Lambda_{n}^{\text {(unit.) }}=n / 2\left|\lambda_{4}^{\text {(unit.) }}\right|$.

We saw in Figure 6.13 that the Sommerfeld enhancement factor varies as $\Lambda_{n}$, so we are not surprised that its $\Omega_{\zeta} / \Omega_{\mathrm{DM}}=1$ curve coincides with the one for the 
no co-annihilations case at $\Lambda_{n}=0$. For large values of $\Lambda_{n}$, the thermally-averaged Sommerfeld enhancement factor can be quite large. This will drive the cross section up, and thus the DM fraction down. Because of this, the range of allowed masses corresponding to $\Omega_{\zeta} / \Omega_{\mathrm{DM}}=1$ is $10.1 \mathrm{TeV} \leq m_{\zeta^{0, r}} \lesssim 325 \mathrm{TeV}$ in $n=6$ and $18.5 \mathrm{TeV} \leq$ $m_{\zeta^{0, r}} \lesssim 400 \mathrm{TeV}$ in $n=8$. We would now like to combine the effects of co-annihilation and Sommerfeld enhancement.

\subsubsection{Combining co-annihilation with Sommerfeld enhancement.}

The simple picture of a multiplicative enhancement factor or a sum of cross sections can no longer be applied when we consider Sommerfeld enhancement with the full large multiplet. For example, if we start with $\zeta^{+3} H_{1}^{-2}$ in the $n=6$ model, then the two initial-state particles might exchange a $W$ boson, changing to $H_{1}^{+2} H_{1}^{-1}$. They could then exchange a $Z$ boson, becoming $H_{2}^{+2} H_{1}^{-1}$. Exchanges of this sort continue until the final particles annihilate into, say, $W^{+} h$. The factorizing of the Sommerfeld effect in the single-particle case cannot be applied to this situation. For the Sommerfeld-enhanced co-annihilation $s_{i} s_{j} \rightarrow s_{k} s_{\ell} \rightarrow \mathrm{SM}_{A} \mathrm{SM}_{B}, s_{k} s_{\ell}$ must run over all possible intermediate states. Instead, we need to consider a matrix version of Equation (6.38) [189, 195, 57, 83,

$$
-\frac{1}{2 \mathcal{M}_{(k \ell)}} \nabla^{2} \psi_{(k \ell)(i j)}^{(A B)}(\vec{r})+V_{(k \ell)(m n)}^{(A B)}(\vec{r}) \psi_{(m n)(i j)}^{(A B)}(\vec{r})=E \psi_{(k \ell)(i j)}^{(A B)}(\vec{r})
$$

where $\mathcal{M}_{(k \ell)}$ is the reduced mass of the intermediate $s_{k} s_{\ell}$ two-particle state. The radial wave-function, $u_{(k \ell)(i j)}^{(A B)}(r)$, satisfies

$$
-\frac{1}{2 \mathcal{M}_{(k \ell)}} \frac{d^{2} u_{(k \ell)(i j)}^{(A B)}(r)}{d r^{2}}+V_{(k \ell)(m n)}^{(A B)}(r) u_{(m n)(i j)}^{(A B)}(\vec{r})=E u_{(k \ell)(i j)}^{(A B)}(r)
$$


where

$$
\psi_{(m n)(i j)}^{(A B)}(\vec{r})=\frac{u_{(k \ell)(i j)}^{(A B)}(r)}{r} Y_{l m}(\theta, \phi) .
$$

Then, the matrix related to the Sommerfeld enhancement factor is given by

$$
s_{(k \ell)(i j)}^{(A B)}=\left.\frac{1}{k_{(k \ell)}^{(A B)}} \frac{d u_{(k \ell)(i j)}^{(A B)}}{d r}\right|_{r=0}
$$

where $k_{(k \ell)}^{(A B)}$ is the wave number of the two-particle state. The Sommerfeld-enhanced co-annihilation for the final state $\mathrm{SM}_{A} \mathrm{SM}_{B}$ will then be proportional to the diagonal elements of

$$
(\sigma \beta)_{(A B)} \propto\left(s^{\dagger} \Gamma s\right)_{(A B)(A B)}
$$

where $\Gamma$ is the annihilation matrix for the short-range process $s_{k} s_{\ell} \rightarrow \mathrm{SM}_{A} \mathrm{SM}_{B}$. The calculation of the Sommerfeld-enhanced co-annihilation cross section in this manner can quickly become intractable. The sheer number of entries in the matrix for our models and numerical integrations required make the computation time daunting. However, for our models, there is a very helpful simplifying approximation.

First we recall from the thermal history described in Section 6.1, the electroweak phase transition (EWPT) occurs at $T_{\mathrm{EWPT}} \sim 0.2 \mathrm{TeV}$ [196, 197]. The mass range of our multi-TeV parameter space, $m \sim(5,50) \mathrm{TeV}$ corresponds to a freeze-out termperature of $T_{\text {f.o. }}=m / x \sim(5 / 20,50 / 20) \mathrm{TeV}=(0.25,2.5) \mathrm{TeV}$. Since $T_{\text {f.o. }}>T_{\text {EwPt }}$ over this whole range, the freeze-out of $\zeta^{0, r}$ occurs before the EWPT-before electroweak symmetry is broken. This means that weak isospin and hypercharge are conserved quantities, they are "good" quantum numbers. In addition, since the Higgs doublet has not yet acquired its vacuum expectation value, the states of $\Sigma$ are degenerate in mass. Furthermore, $W$ and $Z$ are massless, and the $h \Sigma \Sigma$ couplings $(\propto v)$ all go to zero: we can use the Coulomb potential rather than the Yukawa or the Hulthén 
potential. In this case, the potential matrix has elements $V_{\tau \varphi}$, where $\tau$ is the total isospin of the two-particle initial state and $\varphi$ is their total hypercharge. It is given by

$$
V_{\tau \varphi}=\frac{\alpha_{\tau \varphi}}{r}
$$

Whether this potential is repulsive or attractive will depend on the sign of $\alpha_{\tau \varphi}$, as defined below. The coupling parameter $\alpha_{\tau \varphi}$ may be determined for a generic $S U(2)_{L} \otimes U(1)_{Y}$ multiplet from Ref. [198, where we find

$$
\alpha_{\tau \varphi}=\left[2 \tau(\tau+1)+1-n^{2}\right] \frac{g^{2}}{16 \pi}+\eta_{\tau, \varphi} \frac{g^{2}}{16 \pi}
$$

where $\eta_{0,0}=\eta_{1,0}=-1, \eta_{1,2}=+1$, and $\eta_{2,0}=0$. Introduced in Section 4.3, the possible $(\tau, \varphi)$ combinations are shown in Table 6.1. The annihilation amplitudes are given in Equations (4.31), (4.33) and (4.35) and

$$
a_{0}\left([\Sigma \Sigma]_{2} \rightarrow[W W]_{2}\right)=\frac{g^{2}}{16 \pi} \sqrt{\frac{n\left(n^{2}-1\right)\left(n^{2}-4\right)}{30}}
$$

where the isospin-two hypercharge-zero combinations are given by

$$
\begin{aligned}
{[W W]_{2} } & =\frac{1}{\sqrt{3}}\left[\left(W^{+} W^{-}\right)-\sqrt{2}\left(\frac{W^{3} W^{3}}{\sqrt{2}}\right)\right], \\
{\left[\Sigma^{*} \Sigma\right]_{2} } & =\sqrt{\frac{20}{n\left(n^{2}-1\right)\left(n^{2}-4\right)}} \sum_{Q} \zeta^{* Q}\left[T(T+1)-3\left(T^{3}\right)^{2}\right] \zeta^{Q} .
\end{aligned}
$$




\begin{tabular}{cc|c}
\hline \hline$\tau$ & $\varphi$ & $\operatorname{State}(\mathrm{s})$ \\
\hline 0 & 0 & {$\left[\Phi^{*} \Phi\right]_{0},[W W]_{0},[B B]_{0}$} \\
1 & 0 & {$\left[\Phi^{*} \Phi\right]_{1},[W B]_{1}$} \\
1 & 2 & {$[\Phi \Phi]_{1}$} \\
2 & 0 & {$[W W]_{2}$} \\
\hline \hline
\end{tabular}

TABLE 6.1. Total isospin and hypercharge combinations in the SM. These combinations are used in the calculation of the co-annihilating Sommerfeld enhanced relic abundance. The expressions for the amplitudes are given in Equations (4.31), (4.33), (4.35) and (6.60)

The annihilation cross sections are then

$$
\begin{aligned}
& \Gamma_{00}=\frac{8 \pi}{m_{\zeta^{0, r}}^{2}}\left[a_{0}\left(\left[\Sigma^{*} \Sigma\right]_{0} \rightarrow\left[\phi^{*} \phi\right]_{0}\right)^{2}+a_{0}\left(\left[\Sigma^{*} \Sigma\right]_{0} \rightarrow[W W]_{0}\right)^{2}\right. \\
& \left.\quad+a_{0}\left(\left[\Sigma^{*} \Sigma\right]_{0} \rightarrow[B B]_{0}\right)^{2}\right], \\
& \Gamma_{10}=\frac{8 \pi}{m_{\zeta^{0, r}}^{2}}\left[a_{0}\left(\left[\Sigma^{*} \Sigma\right]_{1} \rightarrow\left[\phi^{*} \phi\right]_{1}\right)^{2}+a_{0}\left(\left[\Sigma^{*} \Sigma\right]_{0} \rightarrow[W B]_{1}\right)^{2}\right], \\
& \Gamma_{12}=\frac{8 \pi}{m_{\zeta^{0, r}}^{2}} a_{0}\left([\Sigma \Sigma]_{1} \rightarrow[\phi \phi]_{1}\right)^{2}, \\
& \Gamma_{20}=\frac{8 \pi}{m_{\zeta^{0, r}}^{2}} a_{0}\left([\Sigma \Sigma]_{2} \rightarrow[W W]_{2}\right)^{2} .
\end{aligned}
$$

Recall that we have set $\lambda_{2}=\lambda_{3}=0$ and $\lambda_{4}=(-1)^{\frac{n}{2}} 2 \Lambda_{n} / n$ in these expressions.

Since the exchanged bosons are all massless, we can use the Sommerfeld enhancement factor for the Coulomb potential,

$$
\mathcal{S}_{\tau \varphi}=-\frac{\pi \alpha_{\tau \varphi}}{\beta} \frac{1}{1-\mathrm{e}^{\frac{\pi \alpha_{\tau \varphi}}{\beta}}} .
$$



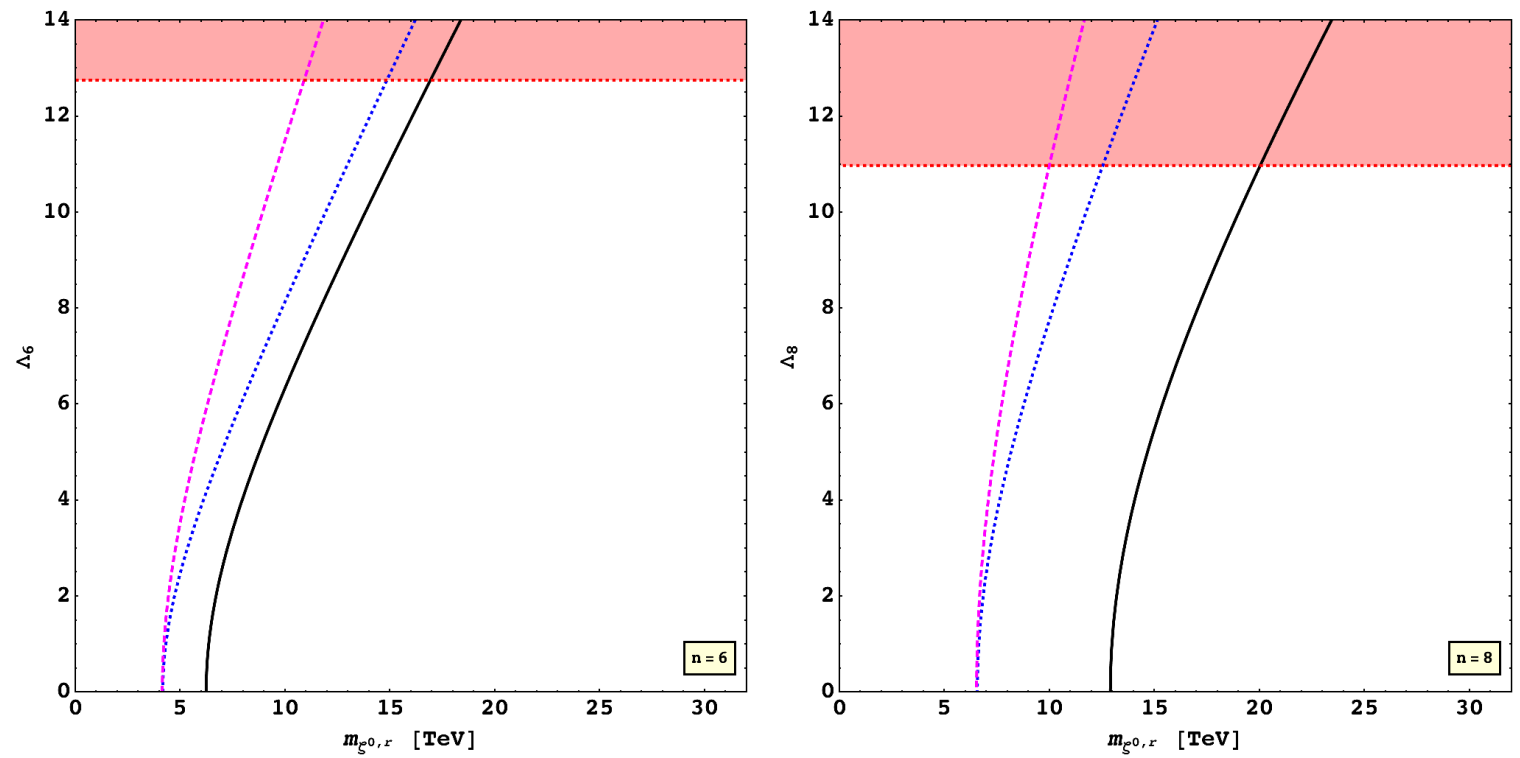

Figure 6.15. Plots showing where $\Omega_{\zeta} / \Omega_{\mathrm{DM}}=1$ comparing coannihliation and Sommerfeld enhancement. The (middle) dotted blue curve corresponds to $\Omega_{\zeta} / \Omega_{\mathrm{DM}}=1$ for co-annihilations as in Figure 6.9. The (left-most) dashed magenta curve corresponds to setting $\mathcal{S}_{\tau \varphi}=1$ in Equation (6.64), The (right-most) solid black curve corresponds to the full expression in Equation (6.64).

Combining this with Equation (6.62), the total Sommerfeld enhanced co-annihilating cross section is given by [199]

$$
\sigma \beta=\frac{2}{(2 n)^{2}} \sum_{\tau, \varphi}(2 \tau+1) \Gamma_{\tau \varphi} \mathcal{S}_{\tau \varphi}
$$

where the factor of 2 in the numerator is due to $\zeta^{0, r}$ being its own antiparticle (this cancels out the $1 / \sqrt{2}$ in the definition of $\zeta^{0, r}$ ) and the factor $2 \tau+1$ is the multiplicity of each isospin state. The factor of $1 /(2 n)^{2}$ accounts for meeting the correct annihilation partner. To determine the DM fraction, we scan over the parameter space, thermally averaging this cross section at each point and comparing to $\langle\sigma \beta\rangle_{\mathrm{STD}}$. We examine the various effects in Figure 6.15. Using Equation (6.28), we get the dotted blue curve, reproducing Figure 6.9. We then take Equation (6.64) and set $\mathcal{S}_{\tau \varphi}=1$, resulting in 
the dashed magenta curve. This shows the effect of setting $v=0$ in the co-annihilation calculation, Equation (6.28), Finally, we plot the full Equation (6.64), which gives the solid black curve in Figure 6.15.

To summarize, in Figure 6.16 , we plot the $\Omega_{\zeta} / \Omega_{\mathrm{DM}}=1$ curves for the four cases of interest. The dashed grey curve is the naïve DM fraction (no co-annihilation and no Sommerfeld enhancement), the dotted blue line is the co-annihilating DM fraction (no Sommerfeld enhancement), the dot-dashed orange curve is the Sommerfeld-enhanced DM fraction (no co-annihilation), and the solid black curve is the Sommerfeld-enhanced co-annihilating cross section. Despite the factor of $1 /(2 n)^{2}$, the Sommerfeld-enhanced co-annihilating DM fraction is decreased from the naïve DM fraction, pushing the required $m_{\zeta^{0, r}}$ higher. The lack of Sommerfeld enhancement from Higgs exchange (since we worked in $T_{\text {f.o. }}>T_{\text {EWPT }}$ ) means that the Sommerfeld enhancement in this situation does not depend on $\Lambda_{n}$, and so is constant for given values of $\tau$ and $\varphi$ $(\langle\mathcal{S}\rangle \sim 2-2.7$ for the values of weak isospin and hypercharge that are required here). In the situation where co-annihilations and Sommerfeld enhancement are both present, we find that $\Omega_{\zeta} / \Omega_{\mathrm{DM}}=1$ corresponds to $6.2 \mathrm{TeV} \leq m_{\zeta^{0, r}} \leq 16.9 \mathrm{TeV}$ in $n=6$ and $12.9 \mathrm{TeV} \leq m_{\zeta^{0, r}} \leq 20.0 \mathrm{TeV}$ in $n=8$.

In summary, then, as $m_{\zeta^{0, r}}$ gets large, we reach a point where $\Omega_{\zeta} / \Omega_{\mathrm{DM}}=1$. In this region, the parameter space is constrained only by perturbative unitarity of $\lambda_{2,3,4}$. However, other effects such as co-annihilation and Sommerfeld enhancement will alter the DM fraction of $\zeta^{0, r}$, leading to different allowed mass regions. We summarize these ranges in Table 6.2. With our parameter space constrained at both the weak-scale and multi-TeV DM mass ranges, we turn now to the direct detection of DM. 

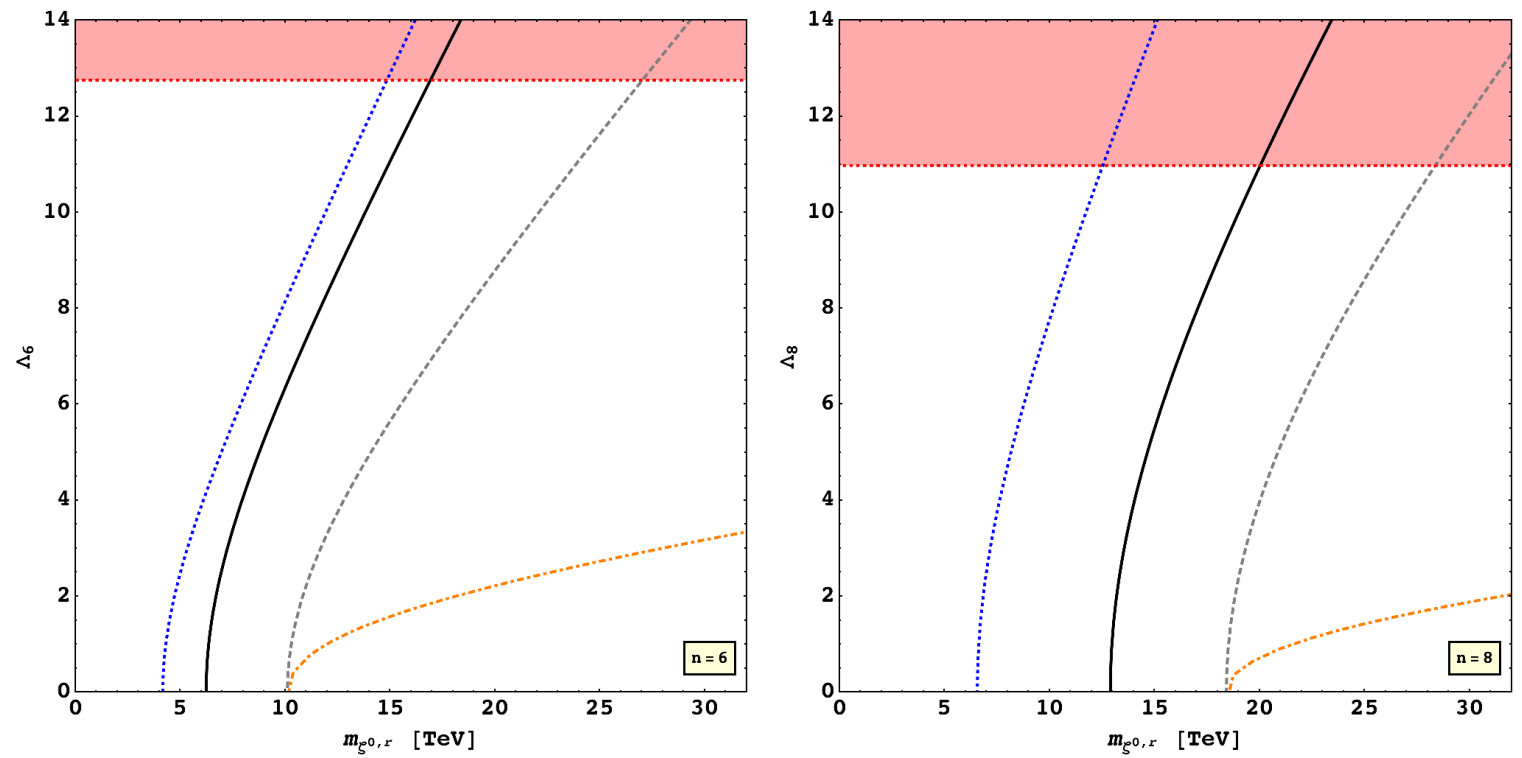

FiguRE 6.16. Plots showing where $\Omega_{\zeta} / \Omega_{\mathrm{DM}}=1$, with $n=6$ on the left and $n=8$ on the right. The solid black line incorporates both coannihilations and Sommerfeld enhancement. The dashed orange line shows Sommerfeld enhancement with only a Higgs potential as in Figure 6.14. The dashed blue line shows co-annihilations as in Figure 6.9. The dashed grey line shows no effects as in Figure 6.6.

\subsection{Dark matter direct detection}

We know that DM exists in the Universe, and we can even say how much of the energy budget of the Universe it should make up. There are many different candidates for DM that run the gamut of masses, couplings, and spins. The typical DM particle velocity in the vicinity of Earth is roughly $\beta \sim 10^{-3}$, which corresponds to a Lorentz boost factor of $\gamma \approx 1.0000005$. In that case, DM interacts with objects on Earth in a very non-relativistic manner. To observe such interactions, we consider the DM scattering elastically off of some nucleus in a detector. This is the simplified view of a DM direct detection experiment: gather together a large quantity of a stable isotope of some heavy (ideally $m_{A} \sim m_{\mathrm{DM}}$ ) element in a background-free environment and wait to see a signal that identifies the rare event of DM scattering off of a nucleus. The 


\begin{tabular}{c|c|c}
\hline \hline Effect & $n=6$ & $n=8$ \\
\hline no effect & {$[10.1,27.0]$} & {$[18.4,28.4]$} \\
co-annihilation & {$[4.2,14.9]$} & {$[6.5,12.5]$} \\
Sommerfeld & {$[10.1,325]$} & {$[18.5,400]$} \\
Sommerfeld and co-annihilation & {$[6.2,16.9]$} & {$[12.9,20.0]$} \\
\hline \hline
\end{tabular}

TABLE 6.2. Mass ranges for multi-TeV $\zeta^{0, r} \mathrm{DM}$. Values given in TeV. The lower-bound on the mass ranges comes from setting $\Lambda_{n}=0$ and the upper-bound comes from setting $\Lambda_{n}=\frac{n}{2}\left|\lambda_{4}^{\text {(unit.) }}\right|$, where the numerical value of $\lambda_{4}^{\text {(unit.) }}$ is given in Table 4.2. In all cases, we set $\lambda_{2,3}=0$. The naïve case considers no additional effects, while the co-annihilations case considers the effects from Section 6.4.1, the Sommerfeld case considers the effects from Section 6.4.2, and finally, the Sommerfeld and co-annihilations case considers the effects from this section.

recoil of the nucleus will produce scintillation light through ionization or excitation as it sheds energy to return to its ground state. For light DM WIMP candidates $\left(m_{\mathrm{DM}} \lesssim 10 \mathrm{GeV}\right)$, experiments such as CDMS low ionization threshold experiment (CDMSlite) [200] and colliders [80] may be used to place limits on the WIMP-nucleon cross section. At higher masses, such as our weak-scale and multi-TeV DM mass large multiplet models, liquid noble gas experiments provide the most stringent limits on the spin-independent cross section. The most sensitive current constraint [201] comes from the Large Underground Xenon (LUX) dark matter experiment, with its full exposure run results expected by the end of 2016. The next generation of (largescale) DM direct detection experiments, such as Dark matter Experiment using Argon Pulse-shape discrimination (DEAP-3600, 3.6 tons of liquid argon, 1 ton of that as fiducial volume) 202] and XENON1T (3.5 tons of liquid xenon, 1 ton of that as fiducial volume) 203, are currently undergoing commissioning and should begin to take data by the end of 2016 . 
Current (and currently-planned) large-scale liquid noble gas (LNG) experiments use isotopes (atoms with the same number of protons, but different numbers of neutrons) of argon or xenon to look for nuclear recoils of WIMP scattering events [204, 205. These events produce scintillation light through excitation or ionization of the detector material. The production method of each light signal differs (summarized in Table 6.3), but each is detected by sensitive light detector such as photomultiplier tubes (PMT) which amplify the small numbers of photons to produce measurable signals. To get scintillation light, first a DM particle hits an atom in the LNG detector, $\mathrm{N}$, changing its kinetic energy. The excited atom then strikes nearby atoms, forming an excited, weakly-bound state (a dimer). This bound state then decays into two ground-state LNG atoms by emitting a (UV) photon. This photon is then detected and hailed as a discovery. Ionization, on the other hand, takes a few extra steps to produce the photon. First, a DM particle hits an atom in the LNG detector, knocking it away from its outermost electron. The ionized atom strikes a nearby neutral atom, forming a charged bound state. This bound state combines with an electron forming a doubly-excited atom and a ground-state atom. The doubly-excited atom will decay to the singly-excited state by emitting some heat. At this point, the excited atom follows the same steps as in the case of scintillation, ending in the production of a photon.

Experiments that use purely liquid argon or xenon (i.e., single-phase detectors) use the shape of the signal as a function of time to determine if the event was a DM WIMP scattering from a nucleus, or just a background event such as electron recoil (excitation of an electron from an incident photon). The different lifetimes of the dimers can distinguish between the signals. They are generally spherical in shape with photomultiplier tubes surrounding the outside of the tank containing the 


\begin{tabular}{cc} 
excitation & ionization \\
\hline $\mathrm{DM}+\mathrm{N} \rightarrow \mathrm{DM}+\mathrm{N}^{*}$ & $\mathrm{DM}+\mathrm{N} \rightarrow \mathrm{DM}+\mathrm{N}^{+}+e^{-}$ \\
$\mathrm{N}^{*}+2 \mathrm{~N} \rightarrow \mathrm{N}_{2}^{*}+\mathrm{N}$ & $\mathrm{N}^{+}+\mathrm{N} \rightarrow \mathrm{N}_{2}^{+}$ \\
$\mathrm{N}_{2}^{*} \rightarrow 2 \mathrm{~N}+\gamma$ & $\mathrm{N}_{2}^{+}+e^{-} \rightarrow \mathrm{N}^{* *}+\mathrm{N}$ \\
& $\mathrm{N}^{* *} \rightarrow \mathrm{N}^{*}+\Delta T$ \\
$\mathrm{~N}^{*}+2 \mathrm{~N} \rightarrow \mathrm{N}_{2}^{*}+\mathrm{N}$ \\
$\mathrm{N}_{2}^{*} \rightarrow 2 \mathrm{~N}+\gamma$
\end{tabular}

TABLE 6.3. Comparison of scintillation light production from excitation or ionization in DM direct detection experiments. In these reactions $\mathrm{N}$ represents a nucleus, $\mathrm{N}^{*}$ represents an excited state of the nucleus, $\mathrm{N}_{2}$ is a bound state of two nuclei, $\Delta T$ represents heat given to the surrounding nuclei, and $\gamma$ are the photons.

LNG. Examples of this type of experiment are DEAP-3600 (argon) [202] and XMASS (xenon) [206].

Experiments that use the noble gas in both its liquid and gaseous forms (dualphase detectors) use the ratio of signal arrival times for scintillation light and electrons to distinguish between WIMP events and background. They are generally cylindrical in shape with photomultiplier tube arrays at the top and bottom of the tank. The electrons will drift to the top of the detector where they interact with a layer of gas, producing scintillation light proportional to the amount of charge. Examples of this type of experiment are ArDM (argon) [207] and LUX (xenon) [201].

As in collider experiments, where the expected number of events depends not only on the underlying event cross section but also the integrated beam luminosity, the number of events at a direct detection experiment will depend on the scattering cross section, the dark matter flux (estimated from the galactic DM density and velocity distribution), and the exposure time of the experiment. The event rate is measured in events per unit time divided by the mass of the detector - the longer an experiment 
runs and the larger the detector target, the more events will be seen for a given cross section. The total exposure is measured in $\mathrm{kg} \times$ day or ton $\times$ year.

If a signal is seen in these experiments, then we can translate this into a cross section based on the rate, $R$. As a function of the nuclear recoil energy, $E_{r}$, the differential rate is given by 204

$$
\frac{d R}{d E_{r}}=N_{T} \frac{\rho_{\mathrm{DM}}}{m_{\mathrm{DM}}} \int_{v_{\text {min }}}^{v_{e s c}} \frac{d \sigma}{d E_{r}}|\vec{v}| f(\vec{v}) d^{3} v,
$$

where $N_{T}$ is the number of target nuclei, $\rho_{\mathrm{DM}}$ and $m_{\mathrm{DM}}$ the local dark matter density and mass, $f(\vec{v})$ is the velocity distribution of the DM within the galactic halo, and $d \sigma / d E_{r}$ is the differential DM-nucleon scattering cross section. The region of integration is defined at the low end by the minimum velocity required to produce a detectable nuclear recoil, and at the high end by the escape velocity of DM from the galactic halo.

Experimental collaborations helpfully provide limits on the per-nucleon cross section as a function of the DM mass? ${ }^{3}$. Currently, the most sensitive constraint is from the LUX experiment [201]. We plot the exclusion curve as a function of DM mass in Figure 6.17. All points above this curve are excluded by the experiment, while those points below are not. The shape of this curve is quite general [205]. The steep loss of sensitivity at low $m_{\mathrm{DM}}$ is due to the low-energy detection threshold of the detector. The minimum depends on the target nucleus through the kinematics of the scattering process. The limit rises linearly in $m_{\mathrm{DM}}$ because, for fixed (known) mass density, the particle number (and hence the flux) falls like $m_{\mathrm{DM}}^{-1}$.

${ }^{3}$ The data for the exclusion curves in all of the direct detection plots in this chapter comes from the DMTools website [208]. 


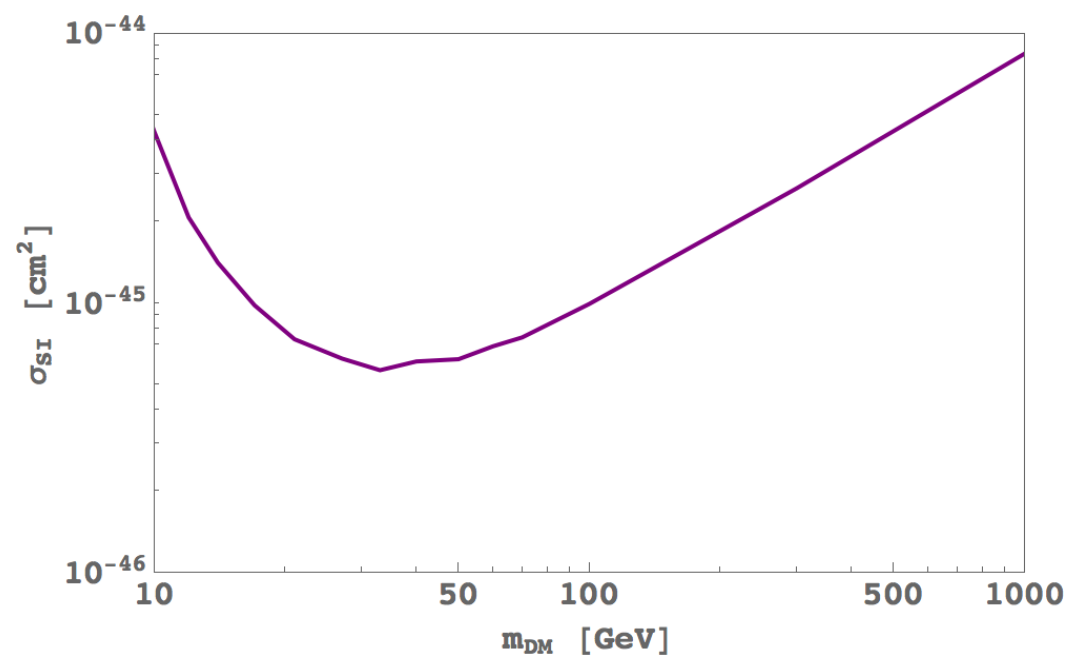

Figure 6.17. Plot of DM direct detection exclusion curve as a function of mass from the LUX collaboration 201. The sharp rise at low $m_{\mathrm{DM}}$ is due to the detector response. The slower rise at high $m_{\mathrm{DM}}$ is due to the rate being suppressed by a factor of $1 / m_{\mathrm{DM}}$. The DM mass corresponding to the lowest point is determined by the target material and the detector's energy threshold.

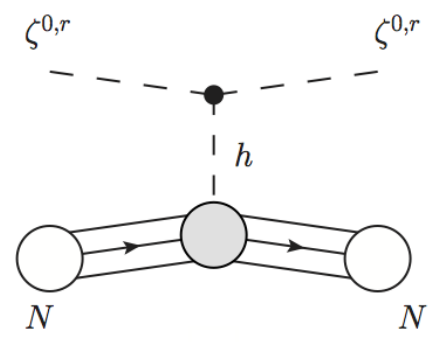

FiguRE 6.18. Feynman diagram for the scattering $\zeta^{0, r} N \rightarrow \zeta^{0, r} N$. The only possible exchange is via a Higgs boson. In this way, the interaction cross section may be set to zero if $\Lambda_{n} \rightarrow 0$.

The particle physics of the interaction between the DM particle and the nucleon is described by a contact interaction, if the DM particles interact directly with gluons or quarks, or by the exchange of some other particle, a scalar or gauge boson, depending on the model. In our case, $\zeta^{0, r}$ communicates with nucleons via the Higgs boson, as shown in Figure 6.18, where $\mathrm{N}$ can be either a proton or a neutron. The interaction 


\begin{tabular}{c|c|c}
\hline \hline$q$ & $f_{T q}^{(p)}$ & $f_{T q}^{(n)}$ \\
\hline$u$ & $0.020 \pm 0.004$ & $0.014 \pm 0.003$ \\
$d$ & $0.026 \pm 0.005$ & $0.036 \pm 0.008$ \\
$s$ & $0.118 \pm 0.062$ & $0.118 \pm 0.062$ \\
\hline
\end{tabular}

TABle 6.4. Effective Higgs-quark couplings. Values from Ref. [209].

between the Higgs and the nucleon is complicated - recall that the proton is made up not only of the three valence quarks, but will also have other partons (gluons and quarks). We may, however, treat it as an effective interaction which provides a vertex $i f_{N}$, where [209, 210]

$$
f_{N}=\frac{m_{N}}{v}\left[\frac{2}{9}+\frac{7}{9} \sum_{q=u, d, s} f_{T q}^{(N)}\right]
$$

and the $f_{T q}^{(N)}$ are given in Table 6.4 and determined using baryon masses [209]. Performing this sum for protons and neutrons, we find

$$
f_{p}=\frac{m_{p}}{v}(0.350 \pm 0.048) \quad, \quad f_{n}=\frac{m_{n}}{v}(0.353 \pm 0.049)
$$

The scattering cross section is then

$$
\sigma_{\mathrm{SI}, N}^{\zeta}=\frac{f_{N}^{2} \Lambda_{n}^{2}}{4 \pi} \frac{v^{2}}{m_{h}^{4}} \frac{m_{N}^{2}}{\left(m_{\zeta^{0, r}}+m_{N}\right)^{2}} .
$$

A large mass of LNG will, in general, contain several different isotopes of the noble gas - atoms with the same number of protons, $Z$, but different numbers of neutrons, $A-Z$, where $A$ is the total number of nucleons. We should average over the abundances present in the target material,

$$
f_{N} \rightarrow \bar{f}_{N}=\frac{Z f_{p}+(A-Z) f_{n}}{A}
$$


However, since $f_{n} \approx f_{p}, \bar{f}_{N} \approx f_{N}$, and we may use $f_{N}=0.35$ in Equation (6.68). We will now discuss the direct detection cross section in the weak-scale and multi-TeV DM mass regions separately.

\subsubsection{Direct detection of weak-scale large multiplet DM.}

Because the relic density is only a small fraction of the total DM density in the weak-scale DM mass region (recall $\Omega_{\zeta} / \Omega_{\mathrm{DM}} \lesssim 1 \%$ ), we must scale the resulting direct detection cross section when comparing to the experimental limits to account for the lower flux of $\zeta^{0, r}$ particles. That is,

$$
\sigma_{\mathrm{SI}}^{\text {expt. }}=\frac{\Omega_{\zeta}}{\Omega_{\mathrm{DM}}} \sigma_{\mathrm{SI}}^{\zeta}
$$

Using the parameter space obtained in Figure 6.4 together with Equation (6.68), we obtain the results shown in Figure 6.19, The colouring in these plots is the same as in Figure 6.4: pale pink regions are allowed by unitarity, STU, $h \rightarrow \gamma \gamma$, and $M^{2}>0$ constraints; light blue regions are allowed by unitarity, $S T U$, and $h \rightarrow \gamma \gamma$ constraints; medium green regions are allowed by unitarity and STU constraints; and dark purple regions are allowed by unitarity constraints only. After all the constraints have been applied, the remaining parameter space sits below the solid black line representing the current best exclusion bound from the Large Underground Xenon (LUX) experiment [201, a dual-phase xenon experiment. The dashed black line shows the expected sensitivity of the DEAP-3600 experiment [202], and the dotted black line shows the expected sensitivity of the XENON1T experiment $[\mathbf{2 0 3}]^{4}$. The dashed grey line shows the expected sensitivity of a proposed 7-ton (5.6-ton fiducial volume) xenon experiment from the merger of the LUX experiment with the ZEPLIN

\footnotetext{
${ }^{4}$ Although DEAP-3600 and XENON1T expect to have nearly the same total exposure, the limits from XENON1T are stronger because $\sigma_{\mathrm{Xe}} / \sigma_{\mathrm{Ar}} \sim A_{\mathrm{Xe}}^{2} / A_{\mathrm{Ar}}^{2} \approx 10$.
} 

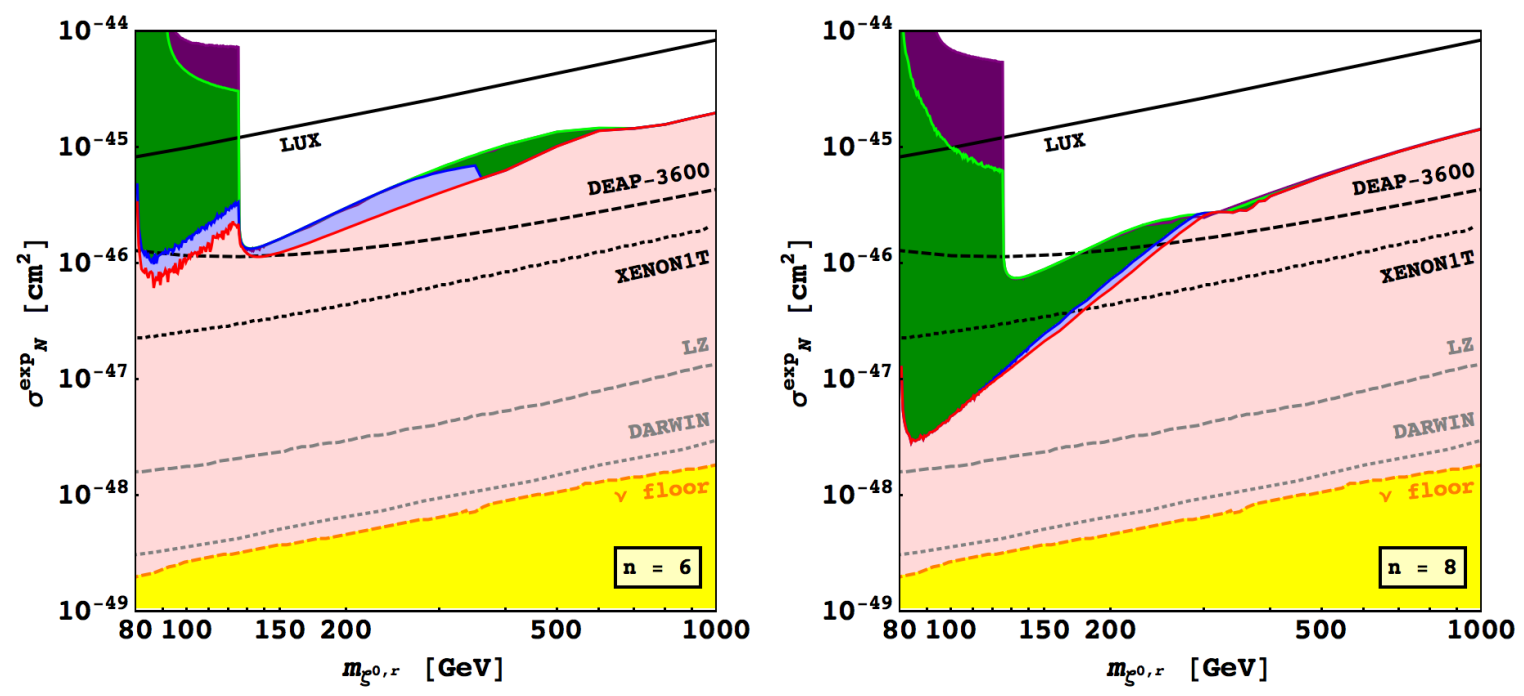

FiguRE 6.19. Direct detection predictions for the weak-scale parameter space. The region allowed by all constraints in these plots is pink. The yellow region shows where coherent neutrino scattering will become a non-negligible background.

experiment, called LZ [211]. Since both DEAP-3600 and XENON1T are currently undergoing the commissioning phase of construction and expecting to start taking data by the end of 2016, we will soon begin to probe the allowed parameter space. The full sensitivity of these experiments is expected in 2019-2020 (the run-time for each experiment is three years). The $\mathrm{LZ}$ experiment is expected to begin construction in 2017, and run for three years once construction is complete. A proposed "ultimate" dark matter direct detection experiment, DARk matter WImp search with liquid xenoN (DARWIN) [212] expects to achieve an ultimate sensitivity of $\sigma_{\text {SI }} \sim 10^{-49} \mathrm{~cm}^{2}$ at $m_{\mathrm{DM}} \sim 50 \mathrm{GeV}$ with a total exposure of $\sim 200$ ton $\times$ year $(50$-tons total volume, 30-tons fiducial). The collaboration is hopeful that a physics run could start as early as 2023 and run for seven years. This projection is shown as the grey dotted line in Figure 6.19. The future experiments will certainly probe a large swath of our allowed parameter space. 
There is, unfortunately, an irreducible background that affects the ultimate sensitivity of DM direct detection experiments: the coherent scattering of neutrinos off the detector material nuclei [213]. For $m_{\mathrm{DM}} \lesssim 10 \mathrm{GeV}$, these background events come from neutrinos which are byproducts of boron- 8 decays and helium-3 neutron capture in the sun, ${ }^{8} \mathrm{~B} \rightarrow{ }^{7} \mathrm{Be}+e^{+}+\nu_{e}$ and ${ }^{3} \mathrm{He}+p \rightarrow{ }^{4} \mathrm{He}+e^{+}+\nu_{e}$. For $m_{\mathrm{DM}} \gtrsim 10 \mathrm{GeV}$, the coherent neutrino scattering background comes from neutrinos produced in cosmic ray collisions in the atmosphere and neutrinos produced in core-collapse supernovae. Events from each of these sources may be mitigated by clever data analysis techniques, but regardless, these neutrinos will become a source of non-negligible backgrounds for very large-scale dark matter direct detection experiments.

\subsubsection{Direct detection of multi-TeV large multiplet DM.}

Finally, we turn to the multi-TeV region of parameter space. Recall that our interest in this region is for $\zeta^{0, r}$ to constitute all of the DM. That means that Equation (6.70) becomes

$$
\sigma_{\mathrm{SI}}^{\mathrm{expt.}}=\sigma_{\mathrm{SI}}^{\zeta}
$$

Using the Sommerfeld-enhanced co-annihilating $\Omega_{\zeta} / \Omega_{\mathrm{DM}}=1$ curve from Figure 6.16, we determine $\Lambda_{n}=\Lambda_{n}\left(m_{\zeta^{0, r}}\right)$ and use this to evaluate Equation (6.68), We plot the resulting curve up to the unitarity bound in Figure 6.20 for $n=6$ (blue curve) and $n=8$ (green curve). The dashed blue and green curves correspond to $\Omega_{\zeta}=$ $\Omega_{\mathrm{DM}} \pm 30 \%$. This accounts for the uncertainty in $\langle\sigma \beta\rangle_{\mathrm{STD}}$.

The exclusion limits provided by experimental collaborations typically only go up to $m_{\mathrm{DM}} \sim 1 \mathrm{TeV}$ (although the limit from LUX extends to $100 \mathrm{TeV}$ [214] and the projection from DEAP-3600 goes up to $12.5 \mathrm{TeV}$ 202]). Because of this, we extrapolate (using a linear fit for the $\sigma_{\mathrm{DD}} \propto m_{\mathrm{DM}}$ rise) up to the multi-TeV mass 
range we are examining. In Figure 6.20, the solid grey line shows the extrapolation of PandaX-II [215], while the solid black line shows the current best exclusion limit from LUX [214], while the dashed and dotted lines correspond to extrapolations of the projected limits. From top (darkest dashed grey) to bottom (lightest dashed grey), they are DEAP-3600 [202], XENON1T [203], LZ [211], and DARWIN [212]. We also plot in orange the neutrino "floor" described at the end of the previous section (this is calculated up to $\sim 10 \mathrm{TeV}$ in Ref. [213] and we extrapolate in the same manner as the other projections; we assume that the slope stays the same over this energy range). In the yellow shaded region below the orange curve, the coherent scattering of neutrinos will become a non-negligible background.

Both the $n=6$ and $n=8$ models evade the current best experimental limits (PandaX-II and LUX). However, the next generation of direct detection experiments (DEAP-3600 and XENON1T) will be able to probe part of the $n=6$ model. Experiments currently in the planning phase (LZ) and proposal stage (DARWIN) will be able to fully probe both the $n=6$ and $n=8$ models up to the neutrino floor. As $\Lambda_{n}$ becomes very small (the mass spectrum of the models is highly compressed), the cross section drops rapidly, and the neutrino background will become non-negligible. The point at which $\sigma_{\mathrm{SI}}^{\zeta}=\sigma_{\nu}$ occurs at roughly $\Lambda_{n} \approx 0.7$.

Finally, we briefly comment on inelastic direct detection scattering processes. In the multi-TeV region, where the mass splittings are small, it may be possible for the $\zeta^{0, r}$ to up-scatter to a $\zeta^{0, i}$, providing an alternative direct detection mechanism through $Z$-boson exchange. At the kinematic threshold, the accessible mass splitting is given in terms of the ambient $\zeta^{0, r}$ velocity $\beta$ by

$$
m_{\zeta^{0, i}}-m_{\zeta^{0, r}} \leq \sqrt{m_{A}^{2}+m_{\zeta^{0, r}}^{2}+2 m_{A} m_{\zeta^{0, r}} \sqrt{1+\beta^{2}}}-m_{A}-m_{\zeta^{0, r}}
$$




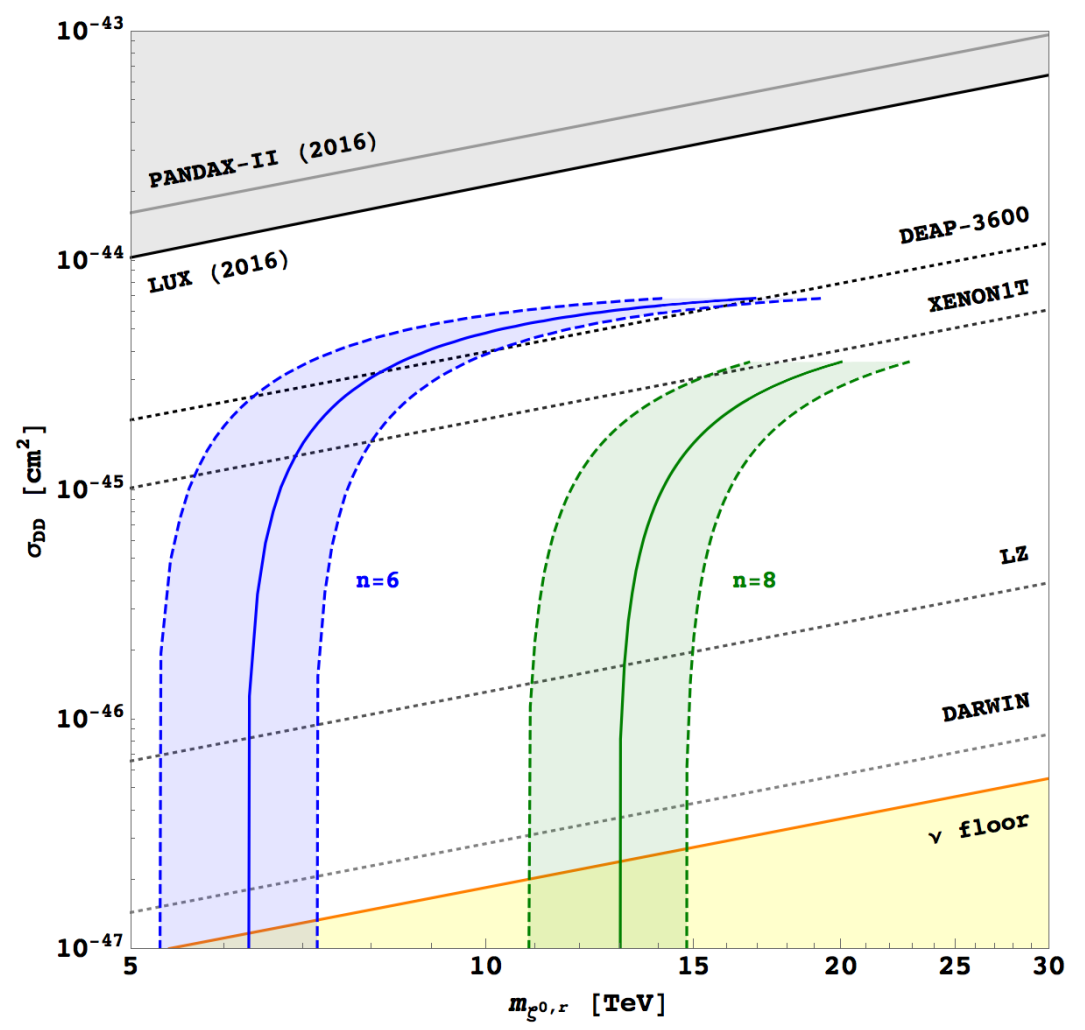

FiguRE 6.20. Direct detection predictions for the multi-TeV parameter space in the $n=6$ (blue) and $n=8$ (green) models. The solid lines show where $\Omega_{\zeta} / \Omega_{\mathrm{DM}}=1$ in each model, incorporating both Sommerfeld enhancement and co-annihilations. The dashed lines on either side show the range of direct detection cross section for $\Omega_{\zeta} / \Omega_{\mathrm{DM}} \in[0.7,1.3]$. The solid grey line shows a projection of the current PandaX-II exclusion curve, while the solid black line shows the current LUX exclusion curve. The dashed grey lines show (from top to bottom) extrapolation of the projections for DEAP-3600, XENON1T, LZ, and DARWIN. The orange curve shows an extrapolation of where coherent neutrino scattering will become a non-negligible background.

where $m_{A}$ is the mass of the scattering target. In our case, the current dark matter velocity is $\beta \sim 10^{-3}$ and the scattering target is a xenon nucleus with $m_{A} \approx 124 \mathrm{GeV}$. For $m_{\zeta^{0, r}} \gg m_{A}$, Equation (6.72) approaches a constant value, $m_{\zeta^{0, i}}-m_{\zeta^{0, r}} \lesssim$ $m_{A^{\beta}} / 2 \sim 60 \mathrm{keV}$. In turn, this implies that the mass splittings between the electricallycharged scalars and $\zeta^{0, r}$ would be much less than the mass of an electron, forcing the 
electrically-charged scalars to be stable. We conclude that the absence of heavy charged relics precludes the possibility of inelastic direct detection scattering in our models.

\subsection{Chapter summary}

In summary, the theoretical and experimental constraints of the previous chapters naturally divide our parameter space into a constrained (weak-scale DM mass) region and an unconstrained (multi-TeV DM mass) region. In the weak-scale region, we find that $\zeta^{0, r}$ is a viable DM candidate, but may only constitute $\lesssim 1 \%$ of the total DM content of the Universe. Though it evades current direct detection exclusion limits, the next generation of direct detection experiments will begin to probe the weak-scale DM mass parameter space. These results were originally published in Ref. [2].

In the multi-TeV region, we demand that $\zeta^{0, r}$ compose all of the DM content of our Universe. In that case, we must consider other effects on the DM fraction than those in the weak-scale region. A combination of unitarity, co-annihilation, and Sommerfeld enhancement sets $m_{\zeta^{0, r}} \in(6.2,16.9) \mathrm{TeV}$ for $n=6$ and $m_{\zeta^{0, r}} \in(12.9,20.0) \mathrm{TeV}$ for $n=8$. Below the unitarity bound, this leads to direct detection cross sections below the current sensitivity of experiments (such as PandaX-II and LUX). Next-generation experiments such as DEAP-3600 and XENON1T will begin to probe the $n=6$ model, but proposed future experiments (such as LZ and DARWIN) will be required to fully explore the parameter space. These results will be published in Ref. [4]. 


\section{CHAPTER 7}

\section{Summary}

The Standard Model of particle physics is incomplete. We see this from such varied corners as the loop corrections to the Higgs mass, the lack of gravity in the model, neutrino oscillations, and the existence of dark matter. For these reasons, we want to find some model of physics that incorporates the SM as a low-energy approximation. This top-down approach would be useful in attempting to address multiple inadequacies at once, but it can be difficult to do in practice. Instead, a bottom-up approach of extending the SM to attack a small number of these problems is often more beneficial.

In our case, we seek to address the problem of dark matter. Astronomical observations such as galactic and cluster rotation curves, and cosmological observations such as the temperature power spectrum of the Cosmic Microwave Background radiation tell us that some form of non-baryonic, non-luminous matter makes up the bulk of the matter content of the Universe. The models we use in this thesis to explain the DM extend the SM by the addition of a single large electroweak multiplet of scalars.

The large multiplet models, if they exist, will scatter into pairs of $S U(2)_{L} \otimes$ $U(1)_{Y}$ gauge bosons. In that regard, they must not violate perturbative unitarity of the scattering amplitude or the theory will become strongly coupled. This bound implies that the size of the large multiplets must be no larger than eight members (equivalently, their total isospin must be $T \leq 7 / 2$ ). Additionally, we impose a $Z_{2}$ 
symmetry to ensure a DM candidate, which restricts the cases of interest to models that have $n=6$ or 8 .

We use theoretical requirements and experimental measurements to constrain the allowed parameter space of our models. To ensure that the scalar potential in our models does not have any minima beyond the SM vacuum expectation value, a sufficient conditions is to require that the bare mass squared be positive, $M^{2}>0$. Using perturbative unitarity of $2 \rightarrow 2$ scalar scattering amplitudes allows us to place upper bounds on the quartic coupling parameters. We use the renormalization group equations to show that these quartic couplings do indeed possess Landau poles, indicating that there is some energy scale beyond which these models are no longer valid descriptions.

Contributions of BSM physics to SM processes at the loop level are constrained by experimental measurements of these SM processes. Electroweak precision measurements give rise to the $S T U$ parameters, which allow us to constrain $\lambda_{3,4}$. Measurements of Higgs decays to two photons place limits on $\lambda_{2}$. Further, we can use direct searches for BSM physics at the LHC to exclude certain regions of parameter space. The most stringent limits arise at low $m_{\zeta^{0, r}}$. Regions where the mass spectrum is highly compressed are less constrained.

If $\zeta^{0, r}$ is a DM candidate, then the constraints on the parameter space of the models divides our interest into a lower mass (weak-scale) region and a higher mass (multi-TeV) region. In the weak-scale region, the previously determined constraints on the parameter space limits $\zeta^{0, r}$ to be no more than $\sim 1 \%$ of the total DM in the Universe for the mass range $m_{\zeta^{0, r}} \in(80,1000) \mathrm{GeV}$. The next generation of direct detection experiments should begin to probe this region. For the multi-TeV region, we would like for $\zeta^{0, r}$ to be all of the DM. In that case, the only constraint that applies 
is perturbative unitarity, but there are two additional important effects that must be considered. The small mass splitting means that co-annihilating states will affect the relic abundance of $\zeta^{0, r}$. In addition, non-perturbative long-range Sommerfeld enhancement will alter the relic abundance. Incorporating these effects sets $\zeta^{0, r}$ as all of the $\mathrm{DM}$ for the mass range $m_{\zeta^{0, r}} \in(6.2,16.9) \mathrm{TeV}$ for $n=6$ and $m_{\zeta^{0, r}} \in$ (12.9, 20.0) TeV for $n=8$. The direct detection cross section that results from these regions is not explored by current experiments such as PandaX-II and LUX. Nextgeneration experiments such as DEAP-3600 and XENON1T will begin to probe the $n=6$ model, but proposed future experiments (such as LZ and DARWIN) will be required to fully explore the parameter space.

Models that extend the SM by a single large electroweak scalar multiplet have rich phenomenology. Throughout this thesis, we see how the effects arising from the large weak charges contribute to strong constraints on the parameter space. In addition, they make enticing targets for direct searches at colliders and DM direct detection experiments. 


\section{APPENDIX A}

\section{Feynman rules of the large electroweak scalar multiplet models}

In this section we collect the Feynman rules for the couplings of the new scalars to gauge and Higgs bosons. We define the couplings with all particles and momenta incoming. For couplings involving scalar momenta, we define $p_{1}$ as the momentum of the first scalar and $p_{2}$ as the momentum of the second scalar.

For simplicity in the derivation of the oblique parameters, all coefficients $C$ for couplings of scalars to one or two electroweak gauge bosons are defined with the overall factors of $e$ removed: one factor of $e$ is removed from couplings to a single gauge boson and two factors of $e$ are removed from couplings to two gauge bosons.

\section{A.1. Higgs boson couplings to scalar pairs}

The Feynman rule for the coupling $h s_{1} s_{2}$ is given by $-i C_{h s_{1} s_{2}}$, where

$$
\begin{aligned}
C_{h \zeta^{0, r} \zeta^{0, r}} & =v\left(\lambda_{2}+\frac{1}{4} \lambda_{3}+\frac{n}{2}(-1)^{\frac{n}{2}+1} \lambda_{4}\right), \\
C_{h \zeta^{0, i} \zeta^{0, i}} & =v\left(\lambda_{2}+\frac{1}{4} \lambda_{3}+\frac{n}{2}(-1)^{\frac{n}{2}} \lambda_{4}\right), \\
C_{h H_{1}^{Q} H_{1}^{-Q}} & =v\left(\lambda_{2}+\frac{1}{4} \lambda_{3}-\frac{1}{2} \sqrt{Q^{2} \lambda_{3}^{2}+\left(n^{2}-4 Q^{2}\right) \lambda_{4}^{2}}\right), \\
C_{h H_{2}^{Q} H_{2}^{-Q}} & =v\left(\lambda_{2}+\frac{1}{4} \lambda_{3}+\frac{1}{2} \sqrt{Q^{2} \lambda_{3}^{2}+\left(n^{2}-4 Q^{2}\right) \lambda_{4}^{2}}\right), \\
C_{h \zeta^{\frac{n}{2} \zeta^{-\frac{n}{2}}}} & =v\left(\lambda_{2}-\frac{2 Q-1}{4} \lambda_{3}\right) .
\end{aligned}
$$


The Feynman rule for the coupling $h h s_{1} s_{2}$ is given by $-i C_{h h s_{1} s_{2}}$, where

$$
\begin{aligned}
C_{h h \zeta^{0, r} \zeta^{0, r}} & =\lambda_{2}+\frac{1}{4} \lambda_{3}+\frac{n}{2}(-1)^{\frac{n}{2}+1} \lambda_{4}, \\
C_{h h \zeta^{0, i} \zeta^{0, i}} & =\lambda_{2}+\frac{1}{4} \lambda_{3}+\frac{n}{2}(-1)^{\frac{n}{2}} \lambda_{4}, \\
C_{h h H_{1}^{Q} H_{1}^{-Q}} & =\lambda_{2}+\frac{1}{4} \lambda_{3}-\frac{1}{2} \sqrt{Q^{2} \lambda_{3}^{2}+\left(n^{2}-4 Q^{2}\right) \lambda_{4}^{2}}, \\
C_{h h H_{2}^{Q} H_{2}^{-Q}} & =\lambda_{2}+\frac{1}{4} \lambda_{3}+\frac{1}{2} \sqrt{Q^{2} \lambda_{3}^{2}+\left(n^{2}-4 Q^{2}\right) \lambda_{4}^{2}}, \\
C_{h h \zeta^{\frac{n}{2}} \zeta^{-\frac{n}{2}}} & =\lambda_{2}-\frac{2 Q-1}{4} \lambda_{3} .
\end{aligned}
$$

Note that, for all the couplings above, $s_{2}=s_{1}^{*}$; i.e., there are no off-diagonal couplings.

\section{A.2. Gauge boson couplings to scalar pairs}

The Feynman rules for the couplings of the new scalars to gauge bosons come from the gauge-kinetic terms in the Lagrangian,

$$
\mathcal{L} \supset\left(\mathcal{D}_{\mu} \Sigma\right)^{\dagger}\left(\mathcal{D}^{\mu} \Sigma\right)
$$

where the covariant derivative is given by

$$
\mathcal{D}_{\mu}=\partial_{\mu}-i \frac{g}{\sqrt{2}}\left(W_{\mu}^{+} T^{+}+W_{\mu}^{-} T^{-}\right)-i \frac{e}{s_{W} c_{W}} Z_{\mu}\left(T^{3}-s_{W}^{2} Q\right)-i e A_{\mu} Q
$$

\section{A.2.1. Couplings to one or two photons.}

The Feynman rule for the coupling $s_{1} s_{2} \gamma_{\mu}$, for $s_{1}$ with charge $Q$ and $s_{2}=s_{1}^{*}$, is

$$
i e C_{s_{1} s_{2} \gamma}\left(p_{1}-p_{2}\right)_{\mu}, \quad \text { where } \quad C_{s_{1} s_{2} \gamma}=Q \text {. }
$$

The Feynman rule for the coupling $s_{1} s_{2} \gamma_{\mu} \gamma_{\nu}$, for $s_{1}$ with charge $Q$ and $s_{2}=s_{1}^{*}$, is

$$
-i e^{2} C_{s_{1} s_{2} \gamma \gamma} g_{\mu \nu}, \quad \text { where } \quad C_{s_{1} s_{2} \gamma \gamma}=-2 Q^{2}
$$


192 A. FEYNMAN RULES OF THE LARGE ELECTROWEAK SCALAR MULTIPLET MODELS

There are no off-diagonal couplings, in accordance with the conservation of the electromagnetic current.

\section{A.2.2. Couplings to one $Z$ boson.}

The Feynman rule for the coupling $s_{1} s_{2} Z_{\mu}$ is given by

$$
i e C_{s_{1} s_{2} Z}\left(p_{1}-p_{2}\right)_{\mu}
$$

where

$$
\begin{aligned}
C_{\zeta^{0, r} \zeta^{0, i} Z} & =\frac{i}{2 s_{W} c_{W}}, \\
C_{H_{1}^{Q} H_{1}^{-Q} Z} & =\frac{1}{s_{W} c_{W}}\left[\left(Q-\frac{1}{2}\right) \cos ^{2} \alpha_{Q}+\left(Q+\frac{1}{2}\right) \sin ^{2} \alpha_{Q}-Q s_{W}^{2}\right], \\
C_{H_{2}^{Q} H_{2}^{-Q} Z} & =\frac{1}{s_{W} c_{W}}\left[\left(Q-\frac{1}{2}\right) \sin ^{2} \alpha_{Q}+\left(Q+\frac{1}{2}\right) \cos ^{2} \alpha_{Q}-Q s_{W}^{2}\right], \\
C_{H_{1}^{Q} H_{2}^{-Q} Z}=C_{H_{2}^{Q} H_{1}^{-Q} Z} & =\frac{1}{s_{W} c_{W}} \sin \alpha_{Q} \cos \alpha_{Q}, \\
C_{\zeta^{\frac{n}{2}} \zeta^{-\frac{n}{2}} Z} & =\frac{1}{s_{W} c_{W}}\left[\frac{n-1}{2}-\frac{n}{2} s_{W}^{2}\right] .
\end{aligned}
$$

Note that the diagonal couplings $C_{\zeta^{0, r} \zeta^{0, r} Z}=C_{\zeta^{0, i} \zeta^{0, i} Z}=0$ due to parity conservation.

\section{A.2.3. Couplings to $Z Z$.}

The Feynman rule for the coupling $s_{1} s_{2} Z_{\mu} Z_{\nu}$ is given by

$$
-i e^{2} C_{s_{1} s_{2} Z Z} g_{\mu \nu}
$$

where

$$
C_{\zeta^{0, r} \zeta^{0, r} Z Z}=C_{\zeta^{0, i} \zeta^{0, i} Z Z}=-\frac{1}{2 s_{W}^{2} c_{W}^{2}}
$$




$$
\begin{gathered}
C_{H_{1}^{Q} H_{1}^{-Q} Z Z}=-\frac{2}{s_{W}^{2} c_{W}^{2}}\left[\left(Q c_{W}^{2}-\frac{1}{2}\right)^{2} \cos ^{2} \alpha_{Q}+\left(Q c_{W}^{2}+\frac{1}{2}\right)^{2} \sin ^{2} \alpha_{Q}\right], \\
C_{H_{1}^{Q} H_{2}^{-Q} Z Z} C_{H_{2}^{Q} H_{1}^{-Q} Z Z}=-\frac{4 Q}{s_{W}^{2} c_{W}^{2}}\left(1-s_{W}^{2}\right) \sin \alpha_{Q} \cos \alpha_{Q}, \\
C_{H_{2}^{Q} H_{2}^{-Q} Z Z}=-\frac{2}{s_{W}^{2} c_{W}^{2}}\left[\left(Q c_{W}^{2}-\frac{1}{2}\right)^{2} \sin ^{2} \alpha_{Q}+\left(Q c_{W}^{2}+\frac{1}{2}\right)^{2} \cos ^{2} \alpha_{Q}\right], \\
C_{\zeta^{\frac{n}{2} \zeta^{-\frac{n}{2}} Z Z}}=-\frac{2}{s_{W}^{2} c_{W}^{2}}\left[\frac{n-1}{2}-\frac{n}{2} s_{W}^{2}\right]^{2} .
\end{gathered}
$$

Note that the off-diagonal coupling $\zeta^{0, r} \zeta^{0, i} Z Z$ is zero.

\section{A.2.4. Couplings to $Z \gamma$.}

The Feynman rule for the coupling $s_{1} s_{2} Z_{\mu} \gamma_{\nu}$ is given by

$$
-i e^{2} C_{s_{1} s_{2} Z \gamma} g_{\mu \nu}
$$

where

$$
\begin{aligned}
& C_{H_{1}^{Q} H_{1}^{-Q} Z_{\gamma}}=-\frac{2 Q}{s_{W} c_{W}}\left[\left(Q-\frac{1}{2}\right) \cos ^{2} \alpha_{Q}+\left(Q+\frac{1}{2}\right) \sin ^{2} \alpha_{Q}-Q s_{W}^{2}\right], \\
& C_{H_{1}^{Q} H_{2}^{-Q} Z_{\gamma}}=C_{H_{2}^{Q} H_{1}^{-Q} Z_{\gamma}}=-\frac{2 Q}{s_{W} c_{W}} \sin \alpha_{Q} \cos \alpha_{Q}, \\
& C_{H_{2}^{Q} H_{2}^{-Q} Z_{\gamma}}=-\frac{2 Q}{s_{W} c_{W}}\left[\left(Q-\frac{1}{2}\right) \sin ^{2} \alpha_{Q}+\left(Q+\frac{1}{2}\right)+\cos ^{2} \alpha_{Q}-Q s_{W}^{2}\right] \text {, } \\
& C_{\zeta^{\frac{n}{2}} \zeta^{-\frac{n}{2}} Z_{\gamma}}=-\frac{n e^{2}}{s_{W} c_{W}}\left[\frac{n-1}{2}-\frac{n}{2} s_{W}^{2}\right]
\end{aligned}
$$

The neutral scalars do not couple to $Z \gamma$.

\section{A.2.5. Couplings to one $W^{ \pm}$boson.}

The Feynman rule for the coupling $s_{1} s_{2} W_{\mu}^{ \pm}$is given by

$$
i e C_{s_{1} s_{2} W^{ \pm}}\left(p_{1}-p_{2}\right)_{\mu}
$$


194 A. FEYNMAN RULES OF THE LARGE ELECTROWEAK SCALAR MULTIPLET MODELS

For compactness, we define the following coefficients for a given value of $n$ :

$$
\begin{aligned}
T_{Q}^{+} & =\frac{1}{2} \sqrt{n^{2}-4 Q^{2}}, \\
T_{Q}^{-} & =\frac{1}{2} \sqrt{n^{2}-4(Q-1)^{2}} .
\end{aligned}
$$

Then the couplings of two scalars to $W^{+}$are given by

$$
\begin{aligned}
C_{\zeta^{0, r} H_{1}^{-} W^{+}} & =\frac{1}{2 s_{W}}\left[\frac{n}{2} \cos \alpha_{1}-T_{-1}^{+} \sin \alpha_{1}\right] \\
C_{\zeta^{0, r} H_{2}^{-} W^{+}} & =\frac{1}{2 s_{W}}\left[-\frac{n}{2} \sin \alpha_{1}-T_{-1}^{+} \cos \alpha_{1}\right] \\
C_{\zeta^{0, i} H_{1}^{-} W^{+}} & =\frac{i}{2 s_{W}}\left[\frac{n}{2} \cos \alpha_{1}+T_{-1}^{+} \sin \alpha_{1}\right] \\
C_{\zeta^{0, i} H_{2}^{-} W^{+}} & =\frac{i}{2 s_{W}}\left[-\frac{n}{2} \sin \alpha_{1}+T_{-1}^{+} \cos \alpha_{1}\right] \\
C_{H_{1}^{Q} H_{1}^{-(Q+1)} W^{+}} & =\frac{1}{\sqrt{2} s_{W}}\left[T_{Q}^{+} \cos \alpha_{Q} \cos \alpha_{Q+1}-T_{-Q-1}^{+} \sin \alpha_{Q} \sin \alpha_{Q+1}\right] \\
C_{H_{1}^{Q} H_{2}^{-(Q+1)} W^{+}} & =\frac{1}{\sqrt{2} s_{W}}\left[-T_{Q}^{+} \cos \alpha_{Q} \sin \alpha_{Q+1}-T_{-Q-1}^{+} \sin \alpha_{Q} \cos \alpha_{Q+1}\right], \\
C_{H_{2}^{Q} H_{1}^{-(Q+1)} W^{+}} & =\frac{1}{\sqrt{2} s_{W}}\left[-T_{Q}^{+} \sin \alpha_{Q} \cos \alpha_{Q+1}-T_{-Q-1}^{+} \cos \alpha_{Q} \sin \alpha_{Q+1}\right], \\
C_{H_{2}^{Q} H_{2}^{-(Q+1)} W^{+}} & =\frac{1}{\sqrt{2} s_{W}}\left[T_{Q}^{+} \sin \alpha_{Q} \sin \alpha_{Q+1}-T_{-Q-1}^{+} \cos \alpha_{Q} \cos \alpha_{Q+1}\right] \\
C_{H_{1}^{\frac{n}{2}-1} \zeta^{-\frac{n}{2}} W^{+}} & =\frac{1}{\sqrt{2} s_{W}} T_{\frac{n}{2}-1}^{+} \cos \alpha_{\frac{n}{2}-1}, \\
C_{H_{2}^{\frac{n}{2}-1} \zeta^{-\frac{n}{2}} W^{+}} & =-\frac{1}{\sqrt{2} s_{W}} T_{\frac{n}{2}-1}^{+} \sin \alpha_{\frac{n}{2}-1} .
\end{aligned}
$$

The couplings of two scalars to $W^{-}$are obtained using the relation

$$
C_{s_{2}^{*} s_{1}^{*} W^{-}}=\left(C_{s_{1} s_{2} W^{+}}\right)^{*}
$$

Note that all the couplings $C_{s_{1} s_{2} W^{+}}$are real except for those that involve one $\zeta^{0, i}$, which are imaginary. 


\section{A.2.6. Couplings to $W^{+} W^{-}$.}

The Feynman rule for the coupling $s_{1} s_{2} W_{\mu}^{+} W_{\nu}^{-}$is given by

$$
-i e^{2} C_{s_{1} s_{2} W^{+} W^{-}} g_{\mu \nu}
$$

For compactness we further define, for a given value of $n$,

$$
\begin{aligned}
& T_{Q}^{+-}=T_{Q}^{+} T_{Q+1}^{-}+T_{Q}^{-} T_{Q-1}^{+}=\frac{n^{2}-2}{2}-2 Q(Q-1), \\
& T_{Q}^{-+}=T_{Q}^{-} T_{Q-1}^{+}+T_{Q}^{+} T_{Q+1}^{-}=\frac{n^{2}-2}{2}-2 Q(Q+1) .
\end{aligned}
$$

Then the couplings of two scalars to $W^{+} W^{-}$are given by

$$
\begin{aligned}
& C_{\zeta^{0, r} \zeta^{0, r} W^{+} W^{-}}=C_{\zeta^{0, i} \zeta^{0, i} W^{+} W^{-}}=-\frac{1}{2 s_{W}^{2}} T_{0}^{+-} \\
& C_{H_{1}^{Q} H_{1}^{-Q} W^{+} W^{-}}=-\frac{1}{2 s_{W}^{2}}\left[T_{Q}^{+-} \cos ^{2} \alpha_{Q}+T_{Q}^{-+} \sin ^{2} \alpha_{Q}\right], \\
& C_{H_{2}^{Q} H_{2}^{-Q} W^{+} W^{-}}=-\frac{1}{2 s_{W}^{2}}\left[T_{Q}^{+-} \sin ^{2} \alpha_{2}+T_{Q}^{-+} \cos ^{2} \alpha_{2}\right], \\
& C_{H_{1}^{Q} H_{2}^{-Q} W^{+} W^{-}}=C_{H_{2}^{Q} H_{1}^{-Q} W^{+} W^{-}}=\frac{2 Q}{s_{W}^{2}} \sin \alpha_{Q} \cos \alpha_{Q}, \\
& C_{\zeta^{\frac{n}{2}} \zeta^{-\frac{n}{2}} W^{+} W^{-}}=-\frac{1}{2 s_{W}^{2}} T_{\frac{n}{2}}^{+-} .
\end{aligned}
$$

Note that the off-diagonal coupling $\zeta^{0, r} \zeta^{0, i} W_{\mu}^{+} W_{\nu}^{-}$is zero.

\section{A.2.7. Couplings to $W^{ \pm} W^{ \pm}$.}

The Feynman rule for the coupling of two scalars to two like-sign $W$ bosons, $s_{1} s_{2} W_{\mu}^{+} W_{\nu}^{+}$, is given by

$$
-i e^{2} C_{s_{1} s_{2} W^{+} W^{+}} g_{\mu \nu},
$$


196 A. FEYNMAN RULES OF THE LARGE ELECTROWEAK SCALAR MULTIPLET MODELS where, for $Q>0$,

$$
\begin{aligned}
& C_{\zeta^{0, r} H_{1}^{--} W^{+} W^{+}}=-\frac{1}{\sqrt{2} s_{W}^{2}}\left[T_{0}^{+} T_{2}^{-} \cos \alpha_{2}+T_{-2}^{+} T_{0}^{-} \sin \alpha_{2}\right] \\
& C_{\zeta^{0, r} H_{2}^{--} W^{+} W^{+}}=-\frac{1}{\sqrt{2} s_{W}^{2}}\left[-T_{0}^{+} T_{2}^{-} \sin \alpha_{2}+T_{-2}^{+} T_{0}^{-} \cos \alpha_{2}\right], \\
& C_{\zeta^{0, i} H_{1}^{--} W^{+} W^{+}}=-\frac{i}{\sqrt{2} s_{W}^{2}}\left[T_{0}^{+} T_{2}^{-} \cos \alpha_{2}-T_{-2}^{+} T_{0}^{-} \sin \alpha_{2}\right] \\
& C_{\zeta^{0, i} H_{2}^{--} W^{+} W^{+}}=-\frac{i}{\sqrt{2} s_{W}^{2}}\left[-T_{0}^{+} T_{2}^{-} \sin \alpha_{2}-T_{-2}^{+} T_{0}^{-} \cos \alpha_{2}\right], \\
& C_{H_{1}^{Q} H_{1}^{-(Q+2)} W^{+} W^{+}}=-\frac{1}{s_{W}^{2}}\left[T_{Q}^{+} T_{Q+2}^{-} \cos \alpha_{Q} \cos \alpha_{Q+2}+T_{-Q-2}^{+} T_{-Q}^{-} \sin \alpha_{Q} \sin \alpha_{Q+2}\right], \\
& C_{H_{2}^{Q} H_{2}^{-(Q+2)} W^{+} W^{+}}=-\frac{1}{s_{W}^{2}}\left[T_{Q}^{+} T_{Q+2}^{-} \sin \alpha_{Q} \sin \alpha_{Q+2}+T_{-Q-2}^{+} T_{-Q}^{-} \cos \alpha_{Q} \cos \alpha_{Q+2}\right], \\
& C_{H_{1}^{Q} H_{2}^{-(Q+2)} W^{+} W^{+}}=-\frac{1}{s_{W}^{2}}\left[-T_{Q}^{+} T_{Q+2}^{-} \cos \alpha_{Q} \sin \alpha_{Q+2}+T_{-Q-2}^{+} T_{-Q}^{-} \sin \alpha_{Q} \cos \alpha_{Q+2}\right] \text {, } \\
& C_{H_{2}^{Q} H_{1}^{-(Q+2)} W^{+} W^{+}}=-\frac{1}{s_{W}^{2}}\left[-T_{Q}^{+} T_{Q+2}^{-} \sin \alpha_{Q} \cos \alpha_{Q+2}+T_{-Q-2}^{+} T_{-Q}^{-} \cos \alpha_{Q} \sin \alpha_{Q+2}\right], \\
& C_{H_{1}^{\frac{n}{2}-2} \zeta^{-\frac{n}{2}} W^{+} W^{+}}=-\frac{1}{s_{W}^{2}} T_{\frac{n}{2}-2}^{+} T_{\frac{n}{2}}^{-} \cos \alpha \frac{n}{2}-2 \\
& C_{H_{2}^{\frac{n}{2}-2} \zeta^{-\frac{n}{2}} W^{+} W^{+}}=\frac{1}{s_{W}^{2}} T_{\frac{n}{2}-2}^{+} T_{\frac{n}{2}}^{-} \sin \alpha_{\frac{n}{2}-2} \\
& C_{H_{1}^{-} H_{1}^{-} W^{+} W^{+}}=-\frac{2}{s_{W}^{2}} T_{1}^{+} T_{1}^{-} \cos \alpha_{1} \sin \alpha_{1}, \\
& C_{H_{2}^{-} H_{2}^{-} W^{+} W^{+}}=\frac{2}{s_{W}^{2}} T_{1}^{+} T_{1}^{-} \cos \alpha_{1} \sin \alpha_{1} \\
& C_{H_{1}^{-} H_{2}^{-} W^{+} W^{+}}=-\frac{1}{s_{W}^{2}} T_{1}^{+} T_{1}^{-}\left(\cos ^{2} \alpha_{1}-\sin ^{2} \alpha_{1}\right) \text {. }
\end{aligned}
$$

The couplings of two scalars to $W^{-} W^{-}$are obtained using the relation

$$
C_{s_{2}^{*} s_{1}^{*} W^{-} W^{-}}=\left(C_{s_{1} s_{2} W^{+} W^{+}}\right)^{*}
$$


Note that all the couplings $C_{s_{1} s_{2} W^{+} W^{+}}$are real except for those that involve one $\zeta^{0, i}$, which are imaginary.

\section{A.2.8. Couplings to $W^{ \pm} \gamma$.}

The Feynman rule for the coupling $s_{1} s_{2} W_{\mu}^{ \pm} \gamma_{\nu}$ is given by

$$
-i e^{2} C_{s_{1} s_{2} W^{ \pm}} g_{\mu \nu}
$$

where

$$
\begin{aligned}
C_{\zeta^{0, r} H_{1}^{-} W^{+\gamma}} & =-\frac{1}{2 s_{W}}\left[\frac{n}{2} \cos \alpha_{1}-T_{-1}^{+} \sin \alpha_{1}\right] \\
C_{\zeta^{0, r} H_{2}^{-} W^{+\gamma}} & =-\frac{1}{2 s_{W}}\left[-\frac{n}{2} \sin \alpha_{1}-T_{-1}^{+} \cos \alpha_{1}\right], \\
C_{\zeta^{0, i} H_{1}^{-} W^{+\gamma}} & =-\frac{i}{2 s_{W}}\left[\frac{n}{2} \cos \alpha_{1}+T_{-1}^{+} \sin \alpha_{1}\right], \\
C_{\zeta^{0, i} H_{1}^{-} W^{+\gamma}} & =-\frac{i}{2 s_{W}}\left[-\frac{n}{2} \sin \alpha_{1}+T_{-1}^{+} \cos \alpha_{1}\right], \\
C_{H_{1}^{Q} H_{1}^{-(Q+1)} W^{+\gamma}} & =-\frac{2 Q+1}{\sqrt{2} s_{W}}\left[T_{Q}^{+} \cos \alpha_{Q} \cos \alpha_{Q+1}-T_{-Q-1}^{+} \sin \alpha_{Q} \sin \alpha_{Q+1}\right], \\
C_{H_{1}^{Q} H_{2}^{-(Q+1)} W^{+\gamma}} & =-\frac{2 Q+1}{\sqrt{2} s_{W}}\left[-T_{Q}^{+} \cos \alpha_{Q} \sin \alpha_{Q+1}-T_{-Q-1}^{+} \sin \alpha_{Q} \cos \alpha_{Q+1}\right], \\
C_{H_{2}^{Q} H_{1}^{-(Q+1)} W^{+\gamma}} & =-\frac{2 Q+1}{\sqrt{2} s_{W}}\left[-T_{Q}^{+} \sin \alpha_{Q} \cos \alpha_{Q+1}-T_{-Q-1}^{+} \cos \alpha_{Q} \sin \alpha_{Q+1}\right], \\
C_{H_{2}^{Q} H_{2}^{-(Q+1)} W^{+\gamma}} & =-\frac{2 Q+1}{\sqrt{2} s_{W}}\left[T_{Q}^{+} \sin \alpha_{Q} \sin \alpha_{Q+1}-T_{-Q-1}^{+} \cos \alpha_{Q} \cos \alpha_{Q+1}\right], \\
C_{H_{1}^{\frac{n}{2}-1} \zeta^{-\frac{n}{2}} W^{+\gamma}} & =-\frac{n-1}{\sqrt{2} s_{W}} T_{\frac{n}{2}-1}^{+} \cos \alpha_{\frac{n}{2}-1}, \\
C_{H_{2}^{\frac{n}{2}-1} \zeta^{-\frac{n}{2}} W^{+\gamma}} & =\frac{n-1}{\sqrt{2} s_{W}} T_{\frac{n}{2}-1}^{+} \sin \alpha_{\frac{n}{2}-1} .
\end{aligned}
$$

The couplings of two scalars to $W^{-} \gamma$ are obtained using the relation

$$
C_{s_{2}^{*} s_{1}^{*} W^{-} \gamma}=\left(C_{s_{1} s_{2} W^{+} \gamma}\right)^{*}
$$


198 A. FEYNMAN RULES OF THE LARGE ELECTROWEAK SCALAR MULTIPLET MODELS

Note that all the couplings $C_{s_{1} s_{2} W^{+} \gamma}$ are real except for those that involve one $\zeta^{0, i}$, which are imaginary.

\section{A.2.9. Couplings to $W^{ \pm} Z$.}

The Feynman rule for the coupling $s_{1} s_{2} W_{\mu}^{ \pm} Z_{\nu}$ is given by

$$
-i e^{2} C_{s_{1} s_{2} W^{ \pm} Z} g_{\mu \nu}
$$

where

$$
\begin{aligned}
& C_{\zeta^{0, r} H_{1}^{-} W^{+} Z}=-\frac{1}{2 s_{W}^{2} c_{W}}\left[-\frac{n}{2} s_{W}^{2} \cos \alpha_{1}-\left(2-s_{W}^{2}\right) T_{-1}^{+} \sin \alpha_{1}\right], \\
& C_{\zeta^{0, r} H_{2}^{-} W^{+} Z}=-\frac{1}{2 s_{W}^{2} c_{W}}\left[\frac{n}{2} s_{W}^{2} \sin \alpha_{1}-\left(2-s_{W}^{2}\right) T_{-1}^{+} \cos \alpha_{1}\right], \\
& C_{\zeta^{0, i} H_{1}^{-} W^{+} Z}=-\frac{i}{2 s_{W}^{2} c_{W}}\left[-\frac{n}{2} s_{W}^{2} \cos \alpha_{1}+\left(2-s_{W}^{2}\right) T_{-1}^{+} \sin \alpha_{1}\right] \text {, } \\
& C_{\zeta^{0, i} H_{2}^{-} W^{+} Z}=-\frac{i}{2 s_{W}^{2} c_{W}}\left[\frac{n}{2} s_{W}^{2} \sin \alpha_{1}+\left(2-s_{W}^{2}\right) T_{-1}^{+} \cos \alpha_{1}\right] \\
& C_{H_{1}^{Q} H_{1}^{-(Q+1)} W^{+} Z}=-\frac{1}{\sqrt{2} s_{W}^{2} c_{W}}\left[-\left(2(Q+1)+(2 Q+1) s_{W}^{2}\right) T_{-Q-1}^{+} \sin \alpha_{Q} \sin \alpha_{Q+1}\right. \\
& \left.+\left(2 Q c_{W}^{2}-s_{W}^{2}\right) T_{Q}^{+} \cos \alpha_{Q} \cos \alpha_{Q+1}\right], \\
& C_{H_{1}^{Q} H_{2}^{-(Q+1)} W^{+} Z}=-\frac{1}{\sqrt{2} s_{W}^{2} c_{W}}\left[-\left(2(Q+1)+(2 Q+1) s_{W}^{2}\right) T_{-Q-1}^{+} \sin \alpha_{Q} \cos \alpha_{Q+1}\right. \\
& \left.-\left(2 Q c_{W}^{2}-s_{W}^{2}\right) T_{Q}^{+} \cos \alpha_{Q} \sin \alpha_{Q+1}\right], \\
& C_{H_{2}^{Q} H_{1}^{-(Q+1)} W^{+Z}}=-\frac{1}{\sqrt{2} s_{W}^{2} c_{W}}\left[-\left(2(Q+1)+(2 Q+1) s_{W}^{2}\right) T_{-Q-1}^{+} \cos \alpha_{Q} \sin \alpha_{Q+1}\right. \\
& \left.-\left(2 Q c_{W}^{2}-s_{W}^{2}\right) T_{Q}^{+} \sin \alpha_{Q} \cos \alpha_{Q+1}\right] \\
& C_{H_{2}^{Q} H_{2}^{-(Q+1)} W^{+} Z}=-\frac{1}{\sqrt{2} s_{W}^{2} c_{W}}\left[-\left(2(Q+1)+(2 Q+1) s_{W}^{2}\right) T_{-Q-1}^{+} \cos \alpha_{Q} \cos \alpha_{Q+1}\right.
\end{aligned}
$$




$$
\begin{array}{r}
\left.+\left(2 Q c_{W}^{2}-s_{W}^{2}\right) T_{Q}^{+} \sin \alpha_{Q} \sin \alpha_{Q+1}\right], \\
C_{H_{1}^{\frac{n}{2}-1} \zeta^{-\frac{n}{2}} W^{+Z}}=-\frac{1}{\sqrt{2} s_{W}^{2} c_{W}}\left(n c_{W}^{2}-2+s_{W}^{2}\right) T_{\frac{n}{2}-1}^{+} \cos \alpha_{\frac{n}{2}-1}, \\
C_{H_{2}^{\frac{n}{2}-1} \zeta^{-\frac{n}{2}} W^{+} Z}=\frac{1}{\sqrt{2} s_{W}^{2} c_{W}}\left(n c_{W}^{2}-2+s_{W}^{2}\right) T_{\frac{n}{2}-1}^{+} \sin \alpha_{\frac{n}{2}-1} .
\end{array}
$$

The couplings of two scalars to $W^{-} Z$ are obtained using the relation

$$
C_{s_{2}^{*} s_{1}^{*} W^{-Z}}=\left(C_{s_{1} s_{2} W^{+} Z}\right)^{*}
$$

Note that all the couplings $C_{s_{1} s_{2} W^{+} Z}$ are real except for those that involve one $\zeta^{0, i}$, which are imaginary. 


\section{APPENDIX B}

\section{Isospin combinations for quartic terms $[\Sigma \Sigma]_{T}$}

We can combine two isospin multiplets with total isospin $T_{(1)}$ and $T_{(2)}$, and third component of isospin $T_{(1)}^{3}$ and $T_{(2)}^{3}$, respectively, into a multiplet with total isospin $T$,

$$
\left|T, T^{3}\right\rangle=\left|T_{(1)}, T_{(1)}^{3}\right\rangle \otimes\left|T_{(2)}, T_{(2)}^{3}\right\rangle
$$

where $T^{3}=T_{(1)}^{3}+T_{(2)}^{3}$ and $T=T_{(1)} \oplus T_{(2)} \in\left[-\left(\left|T_{(1)}\right|+\left|T_{(2)}\right|\right),\left|T_{(1)}\right|+\left|T_{(2)}\right|\right]$. To combine these, act with the usual ladder operators,

$$
\begin{aligned}
& J^{+}|j, m\rangle=\sqrt{j(j+1)-m(m+1)}|j, m+1\rangle, \\
& J^{-}|j, m\rangle=\sqrt{j(j+1)-m(m-1)}|j, m-1\rangle,
\end{aligned}
$$

on the states of Equation (B.1).

To clarify some notation, many of the components of the quartic terms are quite large and will not fit on one page. To simplify presentation, the components will be displayed separately, and so we define the combination with total isospin $T$ as

$$
[\Sigma \Sigma]_{T} \equiv\left(\begin{array}{c}
{[\Sigma \Sigma]_{T}^{(T)}} \\
{[\Sigma \Sigma]_{T}^{(T-1)}} \\
\vdots \\
{[\Sigma \Sigma]_{T}^{(-T+1)}} \\
{[\Sigma \Sigma]_{T}^{(-T)}}
\end{array}\right)
$$


B.1. $n=6$

B.1.1. $[\Phi \Sigma]_{3}$ •

$$
[\Phi \Sigma]_{3}=\left(\begin{array}{c}
\zeta^{+3} \phi^{+} \\
\sqrt{\frac{5}{6}} \zeta^{+2} \phi^{+}+\frac{1}{\sqrt{6}} \zeta^{+3} \phi^{0} \\
\sqrt{\frac{2}{3}} \zeta^{+} \phi^{+}+\frac{1}{\sqrt{3}} \zeta^{+2} \phi^{0} \\
\frac{1}{\sqrt{2}} \zeta^{0} \phi^{+}+\frac{1}{\sqrt{2}} \zeta^{+1} \phi^{0} \\
\frac{1}{\sqrt{3}} \zeta^{-1} \phi^{+}+\sqrt{\frac{2}{3}} \zeta^{0} \phi^{0} \\
\frac{1}{\sqrt{6}} \zeta^{-2} \phi^{+}+\sqrt{\frac{5}{6}} \zeta^{-1} \phi^{0} \\
\zeta^{-2} \phi^{0}
\end{array}\right)
$$

B.1.2. $[\Sigma \Sigma]_{1}$.

$$
\begin{aligned}
{[\Sigma \Sigma]_{1}^{(1)}=} & \frac{1}{\sqrt{7}} \zeta^{-} \zeta^{+3}-\sqrt{\frac{8}{35}} \zeta^{0} \zeta^{+2}+\frac{3}{\sqrt{35}} \zeta^{+} \zeta^{+}-\sqrt{\frac{8}{35}} \zeta^{+2} \zeta^{0}+\frac{1}{\sqrt{7}} \zeta^{+3} \zeta^{-} \\
{[\Sigma \Sigma]_{1}^{(0)}=} & \sqrt{\frac{5}{14}} \zeta^{-2} \zeta^{+3}-\frac{3}{\sqrt{70}} \zeta^{-} \zeta^{+2}+\frac{1}{\sqrt{70}} \zeta^{0} \zeta^{+}+\frac{1}{\sqrt{70}} \zeta^{+} \zeta^{0} \\
& -\frac{3}{\sqrt{70}} \zeta^{+2} \zeta^{-}+\sqrt{\frac{5}{14}} \zeta^{+3} \zeta^{-2}, \\
{[\Sigma \Sigma]_{1}^{(-1)}=} & \frac{1}{\sqrt{7}} \zeta^{-2} \zeta^{+2}-\sqrt{\frac{8}{35}} \zeta^{-} \zeta^{+}+\frac{3}{\sqrt{35}} \zeta^{0} \zeta^{0}-\sqrt{\frac{8}{35}} \zeta^{+} \zeta^{-}+\frac{1}{\sqrt{7}} \zeta^{+2} \zeta^{-2}
\end{aligned}
$$

B.1.3. $[\Sigma \Sigma]_{3}$.

$$
[\Sigma \Sigma]_{3}^{(3)}=\sqrt{\frac{5}{18}} \zeta^{+} \zeta^{+3}-\frac{2}{3} \zeta^{+2} \zeta^{+2}+\sqrt{\frac{5}{18}} \zeta^{+3} \zeta^{+}
$$




$$
\begin{aligned}
{[\Sigma \Sigma]_{3}^{(2)}=} & \sqrt{\frac{5}{12}} \zeta^{0} \zeta^{+3}-\frac{1}{\sqrt{12}} \zeta^{+} \zeta^{+2}-\frac{1}{\sqrt{12}} \zeta^{+2} \zeta^{+}+\sqrt{\frac{5}{12}} \zeta^{+3} \zeta^{0} \\
{[\Sigma \Sigma]_{3}^{(1)}=} & \frac{1}{\sqrt{3}} \zeta^{-} \zeta^{+3}+\frac{1}{\sqrt{30}} \zeta^{0} \zeta^{+2}-\frac{2}{\sqrt{15}} \zeta^{+} \zeta^{+}+\frac{1}{\sqrt{30}} \zeta^{+2} \zeta^{0}+\frac{1}{\sqrt{3}} \zeta^{+3} \zeta^{-} \\
{[\Sigma \Sigma]_{3}^{(0)}=} & \frac{\sqrt{5}}{6} \zeta^{-2} \zeta^{+3}+\frac{7}{6 \sqrt{5}} \zeta^{-} \zeta^{+2}-\frac{2}{3 \sqrt{5}} \zeta^{0} \zeta^{+}-\frac{2}{3 \sqrt{5}} \zeta^{+} \zeta^{0} \\
& +\frac{7}{6 \sqrt{5}} \zeta^{+2} \zeta^{-}+\frac{\sqrt{5}}{6} \zeta^{+3} \zeta^{-2}, \\
{[\Sigma \Sigma]_{3}^{(-1)}=} & \frac{1}{\sqrt{3}} \zeta^{-2} \zeta^{+2}+\frac{1}{\sqrt{30}} \zeta^{-} \zeta^{+}-\frac{2}{\sqrt{15}} \zeta^{0} \zeta^{0}+\frac{1}{\sqrt{30}} \zeta^{+} \zeta^{-}+\frac{1}{\sqrt{3}} \zeta^{+2} \zeta^{-2} \\
{[\Sigma \Sigma]_{3}^{(-2)}=} & \sqrt{\frac{5}{12}} \zeta^{-2} \zeta^{+}-\frac{1}{\sqrt{12}} \zeta^{-} \zeta^{0}-\frac{1}{\sqrt{12}} \zeta^{0} \zeta^{-}+\sqrt{\frac{5}{12}} \zeta^{+} \zeta^{-2} \\
{[\Sigma \Sigma]_{3}^{(-3)}=} & \sqrt{\frac{5}{18}} \zeta^{-2} \zeta^{0}-\frac{2}{3} \zeta^{-} \zeta^{-}+\sqrt{\frac{5}{18}} \zeta^{0} \zeta^{-2},
\end{aligned}
$$

B.1.4. $[\Sigma \Sigma]_{5}$.

$$
\begin{aligned}
{[\Sigma \Sigma]_{5}^{(5)}=} & \zeta^{+3} \zeta^{+3}, \\
{[\Sigma \Sigma]_{5}^{(4)}=} & \frac{1}{\sqrt{2}} \zeta^{+2} \zeta^{+3}+\frac{1}{\sqrt{2}} \zeta^{+3} \zeta^{+2}, \\
{[\Sigma \Sigma]_{5}^{(3)}=} & \frac{\sqrt{2}}{3} \zeta^{+} \zeta^{+3}+\frac{\sqrt{5}}{3} \zeta^{+2} \zeta^{+2}+\frac{\sqrt{2}}{3} \zeta^{+3} \zeta^{+}, \\
{[\Sigma \Sigma]_{5}^{(2)}=} & \frac{1}{2 \sqrt{3}} \zeta^{0} \zeta^{+3}+\frac{1}{2} \sqrt{\frac{5}{3}} \zeta^{+} \zeta^{+2}+\frac{1}{2} \sqrt{\frac{5}{3}} \zeta^{+2} \zeta^{+}+\frac{1}{2 \sqrt{3}} \zeta^{+3} \zeta^{0}, \\
{[\Sigma \Sigma]_{5}^{(1)}=} & \frac{1}{\sqrt{42}} \zeta^{-} \zeta^{+3}+\sqrt{\frac{5}{21}} \zeta^{0} \zeta^{+2}+\sqrt{\frac{10}{21}} \zeta^{+} \zeta^{+}+\sqrt{\frac{5}{21}} \zeta^{+2} \zeta^{0}+\frac{1}{\sqrt{42}} \zeta^{+3} \zeta^{-}, \\
{[\Sigma \Sigma]_{5}^{(0)}=} & \frac{1}{6 \sqrt{7}} \zeta^{-2} \zeta^{+3}+\frac{5}{6 \sqrt{7}} \zeta^{-} \zeta^{+2}+\frac{5}{3 \sqrt{7}} \zeta^{0} \zeta^{+}+\frac{5}{3 \sqrt{7}} \zeta^{+} \zeta^{0} \\
& +\frac{5}{6 \sqrt{7}} \zeta^{+2} \zeta^{-}+\frac{1}{6 \sqrt{7}} \zeta^{+3} \zeta^{-2},
\end{aligned}
$$




$$
\begin{aligned}
& {[\Sigma \Sigma]_{5}^{(-1)}=\frac{1}{\sqrt{42}} \zeta^{-2} \zeta^{+2}+\sqrt{\frac{5}{21}} \zeta^{-} \zeta^{+}+\sqrt{\frac{10}{21}} \zeta^{0} \zeta^{0}+\sqrt{\frac{5}{21}} \zeta^{+} \zeta^{-}+\frac{1}{\sqrt{42}} \zeta^{+2} \zeta^{-2}} \\
& {[\Sigma \Sigma]_{5}^{(-2)}=\frac{1}{2 \sqrt{3}} \zeta^{-2} \zeta^{+}+\frac{1}{2} \sqrt{\frac{5}{3}} \zeta^{-} \zeta^{0}+\frac{1}{2} \sqrt{\frac{5}{3}} \zeta^{0} \zeta^{-}+\frac{1}{2 \sqrt{3}} \zeta^{+} \zeta^{-2}} \\
& {[\Sigma \Sigma]_{5}^{(-3)}=\frac{\sqrt{2}}{3} \zeta^{-2} \zeta^{0}+\frac{\sqrt{5}}{3} \zeta^{-} \zeta^{-}+\frac{\sqrt{2}}{3} \zeta^{0} \zeta^{-2}} \\
& {[\Sigma \Sigma]_{5}^{(-4)}=\frac{1}{\sqrt{2}} \zeta^{-2} \zeta^{-}+\frac{1}{\sqrt{2}} \zeta^{-} \zeta^{-2}} \\
& {[\Sigma \Sigma]_{5}^{(-5)}=\zeta^{-2} \zeta^{-2} .}
\end{aligned}
$$

\section{B.2. $n=8$}

B.2.1. $[\Phi \Sigma]_{3}$.

$$
[\Phi \Sigma]_{3}=\left(\begin{array}{c}
\frac{1}{2} \sqrt{\frac{7}{2}} \zeta^{+4} \phi^{0}-\frac{1}{2 \sqrt{2}} \zeta^{+3} \phi^{+} \\
\frac{1}{2} \sqrt{3} \zeta^{+3} \phi^{0}-\frac{1}{2} \zeta^{+2} \phi^{+} \\
\frac{1}{2} \sqrt{\frac{5}{2}} \zeta^{+2} \phi^{0}-\frac{1}{2} \sqrt{\frac{3}{2}} \zeta^{+} \phi^{+} \\
\frac{1}{\sqrt{2}} \zeta^{+} \phi^{0}-\frac{1}{\sqrt{2}} \zeta^{0} \phi^{+} \\
\frac{1}{2} \sqrt{\frac{3}{2}} \zeta^{0} \phi^{0}-\frac{1}{2} \sqrt{\frac{5}{2}} \zeta^{-} \phi^{+} \\
\frac{1}{2} \zeta^{-} \phi^{0}-\frac{1}{2} \sqrt{3} \zeta^{-2} \phi^{+} \\
\frac{1}{2 \sqrt{2}} \zeta^{-2} \phi^{0}-\frac{1}{2} \sqrt{\frac{7}{2}} \zeta^{-3} \phi^{+}
\end{array}\right) .
$$

B.2.2. $[\Sigma \Sigma]_{1}$.

$$
\begin{aligned}
{[\Sigma \Sigma]_{1}^{(1)}=} & \frac{1}{2 \sqrt{3}} \zeta^{-2} \zeta^{+4}-\frac{1}{\sqrt{7}} \zeta^{-} \zeta^{+3}+\frac{1}{2} \sqrt{\frac{5}{7}} \zeta^{0} \zeta^{+2}-\frac{2}{\sqrt{21}} \zeta^{+} \zeta^{+} \\
& +\frac{1}{2} \sqrt{\frac{5}{7}} \zeta^{+2} \zeta^{0}-\frac{1}{\sqrt{7}} \zeta^{+3} \zeta^{-}+\frac{1}{2 \sqrt{3}} \zeta^{+4} \zeta^{-2} \\
{[\Sigma \Sigma]_{1}^{(0)}=} & \frac{1}{2} \sqrt{\frac{7}{6}} \zeta^{-3} \zeta^{+4}-\frac{5}{2 \sqrt{42}} \zeta^{-2} \zeta^{+3}+\frac{1}{2} \sqrt{\frac{3}{14}} \zeta^{-} \zeta^{+2}-\frac{1}{2 \sqrt{42}} \zeta^{0} \zeta^{+}
\end{aligned}
$$




$$
\begin{aligned}
& -\frac{1}{2 \sqrt{42}} \zeta^{+} \zeta^{0}+\frac{1}{2} \sqrt{\frac{3}{14}} \zeta^{+2} \zeta^{-}-\frac{5}{2 \sqrt{42}} \zeta^{+3} \zeta^{-2}+\frac{1}{2} \sqrt{\frac{7}{6}} \zeta^{+4} \zeta^{-3} \\
{[\Sigma \Sigma]_{1}^{(-1)}=} & \frac{1}{2 \sqrt{3}} \zeta^{-3} \zeta^{+3}-\frac{1}{\sqrt{7}} \zeta^{-2} \zeta^{+2}+\frac{1}{2} \sqrt{\frac{5}{7}} \zeta^{-} \zeta^{+}-\frac{2}{\sqrt{21}} \zeta^{0} \zeta^{0} \\
& +\frac{1}{2} \sqrt{\frac{5}{7}} \zeta^{+} \zeta^{-}-\frac{1}{\sqrt{7}} \zeta^{+2} \zeta^{-2}+\frac{1}{2 \sqrt{3}} \zeta^{+3} \zeta^{-3}
\end{aligned}
$$

B.2.3. $[\Sigma \Sigma]_{3}$.

$$
\begin{aligned}
& {[\Sigma \Sigma]_{3}^{(3)}=\sqrt{\frac{7}{66}} \zeta^{0} \zeta^{+4}-2 \sqrt{\frac{2}{33}} \zeta^{+} \zeta^{+3}+\sqrt{\frac{10}{33}} \zeta^{+2} \zeta^{+2}-2 \sqrt{\frac{2}{33}} \zeta^{+3} \zeta^{+}+\sqrt{\frac{7}{66}} \zeta^{+4} \zeta^{0},} \\
& {[\Sigma \Sigma]_{3}^{(2)}=\frac{1}{2} \sqrt{\frac{35}{33}} \zeta^{-} \zeta^{+4}-\frac{3}{2 \sqrt{11}} \zeta^{0} \zeta^{+3}+\frac{1}{\sqrt{33}} \zeta^{+} \zeta^{+2}+\frac{1}{\sqrt{33}} \zeta^{+2} \zeta^{+}} \\
& -\frac{3}{2 \sqrt{11}} \zeta^{+3} \zeta^{0}+\frac{1}{2} \sqrt{\frac{35}{33}} \zeta^{+4} \zeta^{-}, \\
& {[\Sigma \Sigma]_{3}^{(1)}=\sqrt{\frac{7}{22}} \zeta^{-2} \zeta^{+4}-\frac{1}{\sqrt{66}} \zeta^{-} \zeta^{+3}-\sqrt{\frac{5}{66}} \zeta^{0} \zeta^{+2}+\sqrt{\frac{2}{11}} \zeta^{+} \zeta^{+}} \\
& -\sqrt{\frac{5}{66}} \zeta^{+2} \zeta^{0}-\frac{1}{\sqrt{66}} \zeta^{+3} \zeta^{-}+\sqrt{\frac{7}{22}} \zeta^{+4} \zeta^{-2} \\
& {[\Sigma \Sigma]_{3}^{(0)}=\frac{7}{2 \sqrt{66}} \zeta^{-3} \zeta^{+4}+\frac{5}{2 \sqrt{66}} \zeta^{-2} \zeta^{+3}-\frac{7}{2 \sqrt{66}} \zeta^{-} \zeta^{+2}+\frac{1}{2} \sqrt{\frac{3}{22}} \zeta^{0} \zeta^{+}} \\
& +\frac{1}{2} \sqrt{\frac{3}{22}} \zeta^{+} \zeta^{0}-\frac{7}{2 \sqrt{66}} \zeta^{+2} \zeta^{-}+\frac{5}{2 \sqrt{66}} \zeta^{+3} \zeta^{-2}+\frac{7}{2 \sqrt{66}} \zeta^{+4} \zeta^{-3}, \\
& {[\Sigma \Sigma]_{3}^{(-1)}=\sqrt{\frac{7}{22}} \zeta^{-3} \zeta^{+3}-\frac{1}{\sqrt{66}} \zeta^{-2} \zeta^{+2}-\sqrt{\frac{5}{66}} \zeta^{-} \zeta^{+}+\sqrt{\frac{2}{11}} \zeta^{0} \zeta^{0}} \\
& -\sqrt{\frac{5}{66}} \zeta^{+} \zeta^{-}-\frac{1}{\sqrt{66}} \zeta^{+2} \zeta^{-2}+\sqrt{\frac{7}{22}} \zeta^{+3} \zeta^{-3} \\
& {[\Sigma \Sigma]_{3}^{(-2)}=\frac{1}{2} \sqrt{\frac{35}{33}} \zeta^{-3} \zeta^{+2}-\frac{3}{2 \sqrt{11}} \zeta^{-2} \zeta^{+}+\frac{1}{\sqrt{33}} \zeta^{-} \zeta^{0}+\frac{1}{\sqrt{33}} \zeta^{0} \zeta^{-}} \\
& -\frac{3}{2 \sqrt{11}} \zeta^{+} \zeta^{-2}+\frac{1}{2} \sqrt{\frac{35}{33}} \zeta^{+2} \zeta^{-3}
\end{aligned}
$$




$$
[\Sigma \Sigma]_{3}^{(-3)}=\sqrt{\frac{7}{66}} \zeta^{-3} \zeta^{+}-2 \sqrt{\frac{2}{33}} \zeta^{-2} \zeta^{0}+\sqrt{\frac{10}{33}} \zeta^{-} \zeta^{-}-2 \sqrt{\frac{2}{33}} \zeta^{0} \zeta^{-2}+\sqrt{\frac{7}{66}} \zeta^{+} \zeta^{-3} .
$$

B.2.4. $[\Sigma \Sigma]_{5}$.

$$
\begin{aligned}
& {[\Sigma \Sigma]_{5}^{(5)}=\sqrt{\frac{7}{26}} \zeta^{+2} \zeta^{+4}-\sqrt{\frac{6}{13}} \zeta^{+3} \zeta^{+3}+\sqrt{\frac{7}{26}} \zeta^{+4} \zeta^{+2}} \\
& {[\Sigma \Sigma]_{5}^{(4)}=\frac{1}{2} \sqrt{\frac{21}{13}} \zeta^{+} \zeta^{+4}-\frac{1}{2} \sqrt{\frac{5}{13}} \zeta^{+2} \zeta^{+3}-\frac{1}{2} \sqrt{\frac{5}{13}} \zeta^{+3} \zeta^{+2}+\frac{1}{2} \sqrt{\frac{21}{13}} \zeta^{+4} \zeta^{+}} \\
& {[\Sigma \Sigma]_{5}^{(3)}=\sqrt{\frac{14}{39}} \zeta^{0} \zeta^{+4}+\frac{1}{\sqrt{78}} \zeta^{+} \zeta^{+3}-\sqrt{\frac{10}{39}} \zeta^{+2} \zeta^{+2}+\frac{1}{\sqrt{78}} \zeta^{+3} \zeta^{+}+\sqrt{\frac{14}{39}} \zeta^{+4} \zeta^{0},} \\
& {[\Sigma \Sigma]_{5}^{(2)}=\frac{1}{2} \sqrt{\frac{35}{39}} \zeta^{-} \zeta^{+4}+\frac{3}{2 \sqrt{13}} \zeta^{0} \zeta^{+3}-\frac{2}{\sqrt{39}} \zeta^{+} \zeta^{+2}-\frac{2}{\sqrt{39}} \zeta^{+2} \zeta^{+}} \\
& +\frac{3}{2 \sqrt{13}} \zeta^{+3} \zeta^{0}+\frac{1}{2} \sqrt{\frac{35}{39}} \zeta^{+4} \zeta^{-} \\
& {[\Sigma \Sigma]_{5}^{(1)}=\frac{1}{2} \sqrt{\frac{5}{13}} \zeta^{-2} \zeta^{+4}+4 \sqrt{\frac{5}{273}} \zeta^{-} \zeta^{+3}+\frac{1}{2 \sqrt{273}} \zeta^{0} \zeta^{+2}-2 \sqrt{\frac{5}{91}} \zeta^{+} \zeta^{+}} \\
& +\frac{1}{2 \sqrt{273}} \zeta^{+2} \zeta^{0}+4 \sqrt{\frac{5}{273}} \zeta^{+3} \zeta^{-}+\frac{1}{2} \sqrt{\frac{5}{13}} \zeta^{+4} \zeta^{-2}, \\
& {[\Sigma \Sigma]_{5}^{(0)}=\frac{1}{2} \sqrt{\frac{7}{78}} \zeta^{-3} \zeta^{+4}+\frac{23}{2 \sqrt{546}} \zeta^{-2} \zeta^{+3}+\frac{17}{2 \sqrt{546}} \zeta^{-} \zeta^{+2}-\frac{5}{2} \sqrt{\frac{3}{182}} \zeta^{0} \zeta^{+}} \\
& -\frac{5}{2} \sqrt{\frac{3}{182}} \zeta^{+} \zeta^{0}+\frac{17}{2 \sqrt{546}} \zeta^{+2} \zeta^{-}+\frac{23}{2 \sqrt{546}} \zeta^{+3} \zeta^{-2}+\frac{1}{2} \sqrt{\frac{7}{78}} \zeta^{+4} \zeta^{-3}, \\
& {[\Sigma \Sigma]_{5}^{(-1)}=\frac{1}{2} \sqrt{\frac{5}{13}} \zeta^{-3} \zeta^{+3}+4 \sqrt{\frac{5}{273}} \zeta^{-2} \zeta^{+2}+\frac{1}{2 \sqrt{273}} \zeta^{-} \zeta^{+}-2 \sqrt{\frac{5}{91}} \zeta^{0} \zeta^{0}} \\
& +\frac{1}{2 \sqrt{273}} \zeta^{+} \zeta^{-}+4 \sqrt{\frac{5}{273}} \zeta^{+2} \zeta^{-2}+\frac{1}{2} \sqrt{\frac{5}{13}} \zeta^{+3} \zeta^{-3}, \\
& {[\Sigma \Sigma]_{5}^{(-2)}=\frac{1}{2} \sqrt{\frac{35}{39}} \zeta^{-3} \zeta^{+2}+\frac{3}{2 \sqrt{13}} \zeta^{-2} \zeta^{+}-\frac{2}{\sqrt{39}} \zeta^{-} \zeta^{0}-\frac{2}{\sqrt{39}} \zeta^{0} \zeta^{-}}
\end{aligned}
$$




$$
\begin{aligned}
& +\frac{3}{2 \sqrt{13}} \zeta^{+} \zeta^{-2}+\frac{1}{2} \sqrt{\frac{35}{39}} \zeta^{+2} \zeta^{-3}, \\
& {[\Sigma \Sigma]_{5}^{(-3)}=\sqrt{\frac{14}{39}} \zeta^{-3} \zeta^{+}+\frac{1}{\sqrt{78}} \zeta^{-2} \zeta^{0}-\sqrt{\frac{10}{39}} \zeta^{-} \zeta^{-}+\frac{1}{\sqrt{78}} \zeta^{0} \zeta^{-2}+\sqrt{\frac{14}{39}} \zeta^{+} \zeta^{-3},} \\
& {[\Sigma \Sigma]_{5}^{(-4)}=\frac{1}{2} \sqrt{\frac{21}{13}} \zeta^{-3} \zeta^{0}-\frac{1}{2} \sqrt{\frac{5}{13}} \zeta^{-2} \zeta^{-}-\frac{1}{2} \sqrt{\frac{5}{13}} \zeta^{-} \zeta^{-2}+\frac{1}{2} \sqrt{\frac{21}{13}} \zeta^{0} \zeta^{-3},} \\
& {[\Sigma \Sigma]_{5}^{(-5)}=\sqrt{\frac{7}{26}} \zeta^{-3} \zeta^{-}-\sqrt{\frac{6}{13}} \zeta^{-2} \zeta^{-2}+\sqrt{\frac{7}{26}} \zeta^{-} \zeta^{-3}}
\end{aligned}
$$

\section{B.2.5. $[\Sigma \Sigma]_{7}$.}

$$
\begin{aligned}
& {[\Sigma \Sigma]_{7}^{(7)}=\zeta^{+4} \zeta^{+4}} \\
& {[\Sigma \Sigma]_{7}^{(6)}=\frac{1}{\sqrt{2}} \zeta^{+3} \zeta^{+4}+\frac{1}{\sqrt{2}} \zeta^{+4} \zeta^{+3}} \\
& {[\Sigma \Sigma]_{7}^{(5)}=\sqrt{\frac{3}{13}} \zeta^{+2} \zeta^{+4}+\sqrt{\frac{7}{13}} \zeta^{+3} \zeta^{+3}+\sqrt{\frac{3}{13}} \zeta^{+4} \zeta^{+2}} \\
& {[\Sigma \Sigma]_{7}^{(4)}=\frac{1}{2} \sqrt{\frac{5}{13}} \zeta^{+} \zeta^{+4}+\frac{1}{2} \sqrt{\frac{21}{13}} \zeta^{+2} \zeta^{+3}+\frac{1}{2} \sqrt{\frac{21}{13}} \zeta^{+3} \zeta^{+2}+\frac{1}{2} \sqrt{\frac{5}{13}} \zeta^{+4} \zeta^{+},} \\
& {[\Sigma \Sigma]_{7}^{(3)}=\sqrt{\frac{5}{143}} \zeta^{0} \zeta^{+4}+\sqrt{\frac{35}{143}} \zeta^{+} \zeta^{+3}+\sqrt{\frac{63}{143}} \zeta^{+2} \zeta^{+2}+\sqrt{\frac{35}{143}} \zeta^{+3} \zeta^{+}+\sqrt{\frac{5}{143}} \zeta^{+4} \zeta^{0},} \\
& {[\Sigma \Sigma]_{7}^{(2)}=\sqrt{\frac{3}{286}} \zeta^{-} \zeta^{+4}+\sqrt{\frac{35}{286}} \zeta^{0} \zeta^{+3}+\sqrt{\frac{105}{286}} \zeta^{+} \zeta^{+2}+\sqrt{\frac{105}{286}} \zeta^{+2} \zeta^{+}} \\
& +\sqrt{\frac{35}{286}} \zeta^{+3} \zeta^{0}+\sqrt{\frac{3}{286}} \zeta^{+4} \zeta^{-}, \\
& {[\Sigma \Sigma]_{7}^{(1)}=\frac{1}{\sqrt{429}} \zeta^{-2} \zeta^{+4}+\sqrt{\frac{7}{143}} \zeta^{-} \zeta^{+3}+\sqrt{\frac{35}{143}} \zeta^{0} \zeta^{+2}+5 \sqrt{\frac{7}{429}} \zeta^{+} \zeta^{+}} \\
& +\sqrt{\frac{35}{143}} \zeta^{+2} \zeta^{0}+\sqrt{\frac{7}{143}} \zeta^{+3} \zeta^{-}+\frac{1}{\sqrt{429}} \zeta^{+4} \zeta^{-2}, \\
& {[\Sigma \Sigma]_{7}^{(0)}=\frac{1}{2 \sqrt{858}} \zeta^{-3} \zeta^{+4}+\frac{7}{2 \sqrt{858}} \zeta^{-2} \zeta^{+3}+\frac{7}{2} \sqrt{\frac{3}{286}} \zeta^{-} \zeta^{+2}+\frac{35}{2 \sqrt{858}} \zeta^{0} \zeta^{+}}
\end{aligned}
$$




$$
\begin{aligned}
&+\frac{35}{2 \sqrt{858}} \zeta^{+} \zeta^{0}+\frac{7}{2} \sqrt{\frac{3}{286}} \zeta^{+2} \zeta^{-}+\frac{7}{2 \sqrt{858}} \zeta^{+3} \zeta^{-2}+\frac{1}{2 \sqrt{858}} \zeta^{+4} \zeta^{-3}, \\
& {[\Sigma \Sigma]_{7}^{(-1)}=} \frac{1}{\sqrt{429}} \zeta^{-3} \zeta^{+3}+\sqrt{\frac{7}{143}} \zeta^{-2} \zeta^{+2}+\sqrt{\frac{35}{143}} \zeta^{-} \zeta^{+}+5 \sqrt{\frac{7}{429}} \zeta^{0} \zeta^{0} \\
&+\sqrt{\frac{35}{143}} \zeta^{+} \zeta^{-}+\sqrt{\frac{7}{143}} \zeta^{+2} \zeta^{-2}+\frac{1}{\sqrt{429}} \zeta^{+3} \zeta^{-3}, \\
& {[\Sigma \Sigma]_{7}^{(-2)}=} \sqrt{\frac{3}{286}} \zeta^{-3} \zeta^{+2}+\sqrt{\frac{35}{286}} \zeta^{-2} \zeta^{+}+\sqrt{\frac{105}{286}} \zeta^{-} \zeta^{0}+\sqrt{\frac{105}{286}} \zeta^{0} \zeta^{-} \\
&+\sqrt{\frac{35}{286}} \zeta^{+} \zeta^{-2}+\sqrt{\frac{3}{286}} \zeta^{+2} \zeta^{-3}, \\
& {[\Sigma \Sigma]_{7}^{(-3)}=} \sqrt{\frac{5}{143}} \zeta^{-3} \zeta^{+}+\sqrt{\frac{35}{143}} \zeta^{-2} \zeta^{0}+\sqrt{\frac{63}{143}} \zeta^{-} \zeta^{-}+\sqrt{\frac{35}{143}} \zeta^{0} \zeta^{-2}+\sqrt{\frac{5}{143}} \zeta^{+} \zeta^{-3} \\
& {[\Sigma \Sigma]_{7}^{(-4)}=} \frac{1}{2} \sqrt{\frac{5}{13}} \zeta^{-3} \zeta^{0}+\frac{1}{2} \sqrt{\frac{21}{13}} \zeta^{-2} \zeta^{-}+\frac{1}{2} \sqrt{\frac{21}{13}} \zeta^{-} \zeta^{-2}+\frac{1}{2} \sqrt{\frac{5}{13}} \zeta^{0} \zeta^{-3}, \\
& {[\Sigma \Sigma]_{7}^{(-5)}=} \sqrt{\frac{3}{13}} \zeta^{-3} \zeta^{-}+\sqrt{\frac{7}{13}} \zeta^{-2} \zeta^{-2}+\sqrt{\frac{3}{13}} \zeta^{-} \zeta^{-3}, \\
& {[\Sigma \Sigma]_{7}^{(-6)}=} \frac{1}{\sqrt{2} \zeta^{-3}} \zeta^{-2}+\frac{1}{\sqrt{2}} \zeta^{-2} \zeta^{-3}, \\
& {[\Sigma \Sigma]_{7}^{(-7)}=} \zeta^{-3} \zeta^{-3} \\
& {[\mathrm{~B} .12) }
\end{aligned}
$$




\section{APPENDIX C}

\section{Larger-dimensional representations of $S U(2)$}

We present here the definitions of the conjugation matrix and generators for $n$ dimensional ( $n \leq 8$ or, equivalently, $T \leq 7 / 2$ ) representations of $S U(2)$. The conjugation matrix is used in terms of the form $\widetilde{x}=C x^{*}$ which appear in the $Y_{\Sigma}=1$ version of the Higgs potential. These matrices are anti-diagonal. This can be compactly written as $A \equiv \operatorname{anti-diag}\left(a_{1, n}, a_{2, n-1}, a_{3, n-2}, \cdots, a_{n-1,2}, a_{n, 1}\right)$. The $T^{a}$ are the standard $S U(2)$ generators and are given here for completeness. $T^{ \pm}$can be calculated from $T^{1,2}$ (and vice versa) using the relations

$$
\begin{array}{cc}
T^{+}=\frac{1}{2}\left(T^{1}+i T^{2}\right), & T^{-}=\frac{1}{2}\left(T^{1}-i T^{2}\right) . \\
\text { C.1. } n=2(T=1 / 2) & T^{2}=-\frac{i}{2}\left(\begin{array}{cc}
0 & 1 \\
-1 & 0
\end{array}\right), \\
T^{1}=\frac{1}{2}\left(\begin{array}{cc}
0 & 1 \\
1 & 0
\end{array}\right), & C=\left(\begin{array}{cc}
0 & 1 \\
-1 & 0
\end{array}\right) .
\end{array}
$$


C.2. $n=3(T=1)$

$$
\begin{aligned}
& T^{1}=\frac{1}{2}\left(\begin{array}{ccc}
0 & \sqrt{2} & 0 \\
\sqrt{2} & 0 & \sqrt{2} \\
0 & \sqrt{2} & 0
\end{array}\right), \quad T^{2}=-\frac{i}{2}\left(\begin{array}{ccc}
0 & \sqrt{2} & 0 \\
-\sqrt{2} & 0 & \sqrt{2} \\
0 & -\sqrt{2} & 0
\end{array}\right) \\
& T^{3}=\left(\begin{array}{ccc}
1 & 0 & 0 \\
0 & 0 & 0 \\
0 & 0 & -1
\end{array}\right), \quad C=\left(\begin{array}{ccc}
0 & 0 & 1 \\
0 & -1 & 0 \\
1 & 0 & 0
\end{array}\right) . \\
& \text { C.3. } n=4(T=3 / 2) \\
& T^{1}=\frac{1}{2}\left(\begin{array}{cccc}
0 & \sqrt{3} & 0 & 0 \\
\sqrt{3} & 0 & 2 & 0 \\
0 & 2 & 0 & \sqrt{3} \\
0 & 0 & \sqrt{3} & 0
\end{array}\right), \\
& T^{2}=-\frac{i}{2}\left(\begin{array}{cccc}
0 & \sqrt{3} & 0 & 0 \\
-\sqrt{3} & 0 & 2 & 0 \\
0 & -2 & 0 & \sqrt{3} \\
0 & 0 & -\sqrt{3} & 0
\end{array}\right) \text {, } \\
& T^{3}=\left(\begin{array}{cccc}
3 & 0 & 0 & 0 \\
0 & 1 & 0 & 0 \\
0 & 0 & -1 & 0 \\
0 & 0 & 0 & -3
\end{array}\right), \\
& C=\left(\begin{array}{cccc}
0 & 0 & 0 & 1 \\
0 & 0 & -1 & 0 \\
0 & 1 & 0 & 0 \\
-1 & 0 & 0 & 0
\end{array}\right) \text {. }
\end{aligned}
$$


C.4. $n=5(T=2)$

$$
T^{1}=\frac{1}{2}\left(\begin{array}{ccccc}
0 & & & \\
2 & 0 & & \text { sym. } \\
0 & \sqrt{6} & 0 & \\
0 & 0 & \sqrt{6} & 0 & \\
0 & 0 & 0 & 2 & 0
\end{array}\right), T^{2}=-\frac{i}{2}\left(\begin{array}{ccccc}
0 & & & \\
-2 & 0 & & \text { anti-sym. } \\
0 & -\sqrt{6} & 0 & \\
0 & 0 & -\sqrt{6} & 0 & \\
0 & 0 & 0 & -2 & 0
\end{array}\right),
$$$$
T^{3}=\operatorname{diag}(2,1,0,-1,-2), \quad C=\operatorname{anti-diag}(1,-1,1,-1,1) .
$$

$$
\text { C.5. } n=6(T=5 / 2)
$$

$$
T^{1}=\frac{1}{2}\left(\begin{array}{cccccc}
0 & & & & & \\
\sqrt{5} & 0 & & \text { sym. } & & \\
0 & \sqrt{8} & 0 & & & \\
0 & 0 & 3 & 0 & & \\
0 & 0 & 0 & \sqrt{8} & 0 & \\
0 & 0 & 0 & 0 & \sqrt{5} & 0
\end{array}\right),
$$




$$
\begin{aligned}
T^{3} & =\frac{1}{2} \operatorname{diag}(5,3,1,-1,-3,-5), \\
C & =\operatorname{anti-diag}(1,-1,1,-1,1,-1) .
\end{aligned}
$$

$$
\text { C.6. } n=7(T=3)
$$

$T^{1}=\frac{1}{2}\left(\begin{array}{cccccccc}0 & & & & & & \\ \sqrt{6} & 0 & & & & & \\ 0 & \sqrt{10} & 0 & & \text { sym. } & & \\ 0 & 0 & \sqrt{12} & 0 & & & \\ 0 & 0 & 0 & \sqrt{12} & 0 & & \\ 0 & 0 & 0 & 0 & \sqrt{10} & 0 & \\ 0 & 0 & 0 & 0 & 0 & \sqrt{6} & 0\end{array}\right)$

$T^{2}=-\frac{i}{2}\left(\begin{array}{ccccccc}0 & & & & & & \\ -\sqrt{6} & 0 & & & & \\ 0 & -\sqrt{10} & 0 & & & & \\ 0 & 0 & -\sqrt{12} & 0 & & & \\ 0 & 0 & 0 & -\sqrt{12} & 0 & \\ 0 & 0 & 0 & 0 & -\sqrt{10} & 0 & \\ 0 & 0 & 0 & 0 & 0 & -\sqrt{6} & 0\end{array}\right)$

$T^{3}=\operatorname{diag}(3,1,0,-1,-3)$,

$$
C=\operatorname{anti-diag}(1,-1,1,-1,1,-1,1) .
$$




$$
\text { C.7. } n=8(T=7 / 2)
$$

$T^{1}=\frac{1}{2}\left(\begin{array}{ccccccccc}0 & & & & & & & \\ \sqrt{7} & 0 & & & & & & \\ 0 & \sqrt{12} & 0 & & & \text { sym. } & & \\ 0 & 0 & \sqrt{15} & 0 & & & & \\ 0 & 0 & 0 & 4 & 0 & & & \\ 0 & 0 & 0 & 0 & \sqrt{15} & 0 & & \\ 0 & 0 & 0 & 0 & 0 & \sqrt{12} & 0 & \\ 0 & 0 & 0 & 0 & 0 & 0 & \sqrt{7} & 0\end{array}\right)$,

$T^{2}=-\frac{i}{2}\left(\begin{array}{ccccccccc}0 & & & & & & & \\ -\sqrt{7} & 0 & & & & & \\ 0 & -\sqrt{12} & 0 & \multicolumn{5}{c}{\text { anti-sym. }} \\ 0 & 0 & -\sqrt{15} & 0 & & & & \\ 0 & 0 & 0 & -4 & 0 & & & \\ 0 & 0 & 0 & 0 & -\sqrt{15} & 0 & & \\ 0 & 0 & 0 & 0 & 0 & -\sqrt{12} & 0 & \\ 0 & 0 & 0 & 0 & 0 & 0 & -\sqrt{7} & 0\end{array}\right)$

$$
\begin{aligned}
T^{3} & =\frac{1}{2} \operatorname{diag}(7,5,3,1,-1,-3,-5,-7), \\
C & =\operatorname{anti-diag}(1,-1,1,-1,1,-1,1,-1) .
\end{aligned}
$$




\section{APPENDIX D}

\section{One-loop RGEs in the large electroweak scalar multiplet models}

In this appendix, we provide the full one-loop renormalization group equations (RGEs) for the large multiplet models. We give the expressions for the mass parameters, $\mu_{h}^{2}$ and $M^{2}$, as well as for the gauge couplings, $g_{i}$, and the scalar quartic couplings, $\lambda_{i}$. The derivation for the RGEs of scalar quartic couplings and the mass parameters is given in Section 4.4.

The expression for the $U(1)_{Y}$ gauge coupling in a large multiplet model, $g_{1}$, is given by [122],

$$
(4 \pi)^{2} \frac{d g_{1}}{d t}=\left[\frac{41}{10}+\frac{1}{20} n Y_{\Sigma}^{2}\right] g_{1}^{3},
$$

where $n$ is the size of the multiplet and $Y_{\Sigma}$ is the multiplet's hypercharge. We use the GUT normalization such that $g^{\prime}=\sqrt{\frac{3}{5}} g_{1}, g=g_{2}$, and $g_{s}=g_{3}$.

The expression for the $S U(2)_{L}$ gauge coupling in a large multiplet model, $g_{2}$ is given by [216],

$$
(4 \pi)^{2} \frac{d g_{2}}{d t}=-\left[\frac{19}{6}-\frac{n\left(n^{2}-1\right)}{36}\right] g_{2}^{3},
$$

where $n$ is the size of the multiplet and $Y_{i}$ is the multiplet's hypercharge.

The expression for the $S U(3)_{c}$ gauge coupling, $g_{3}$ is unchanged by the presence of a large $S U(2)_{L} \otimes U(1)_{Y}$ multiplet, and is the same as in the SM, given by [112],

$$
(4 \pi)^{2} \frac{d g_{3}}{d t}=-\left[11-\frac{2}{3} n_{f}\right] g_{3}^{3},
$$



where $n_{f}$ is the number of coloured fermions (in our case, $n_{f}=6$ ).

$$
\begin{aligned}
& \text { D.1. } n=6 \\
& 16 \pi^{2} \frac{d g_{1}}{d t}=\frac{22}{5} g_{1}^{3} \\
& 16 \pi^{2} \frac{d g_{2}}{d t}=\frac{8}{3} g_{2}^{3} \\
& 16 \pi^{2} \frac{d g_{3}}{d t}=-7 g_{3}^{3} \\
& 16 \pi^{2} \frac{d \mu_{h}^{2}}{d t}=-\frac{9}{10} g_{1}^{2} \mu_{h}^{2}-\frac{9}{2} g_{2}^{2} \mu_{h}^{2}+12 \lambda_{1} \mu_{h}^{2}+12 \lambda_{2} M^{2}+6 y_{t}^{2} \mu_{h}^{2}+6 y_{b}^{2} \mu_{h}^{2}+2 y_{\tau}^{2} \mu_{h}^{2}, \\
& 16 \pi^{2} \frac{d M^{2}}{d t}=-\frac{9}{10} g_{1}^{2} M^{2}-\frac{105}{2} g_{2}^{2} M^{2}+4 \lambda_{2} \mu_{h}^{2}+4 \lambda_{6} M^{2}+\frac{28}{3} \lambda_{7} M^{2}+\frac{44}{3} \lambda_{8} M^{2}, \\
& 16 \pi^{2} \frac{d \lambda_{1}}{d t}=\frac{27}{200} g_{1}^{4}+\frac{9}{8} g_{2}^{4}+\frac{9}{20} g_{1}^{2} g_{2}^{2}-\frac{9}{5} g_{1}^{2} \lambda_{1}-9 g_{2}^{2} \lambda_{1}+24 \lambda_{1}^{2}+6 \lambda_{2}^{2}+\frac{35}{8} \lambda_{3}^{2} \\
& +35 \lambda_{4}^{2}+12 y_{t}^{2} \lambda_{1}+12 y_{b}^{2} \lambda_{1}+4 y_{\tau}^{2} \lambda_{1}-6 y_{t}^{4}-6 y_{b}^{4}-2 y_{\tau}^{4} \text {, } \\
& 16 \pi^{2} \frac{d \lambda_{2}}{d t}=\frac{27}{100} g_{1}^{4}+\frac{105}{4} g_{2}^{4}-\frac{9}{5} g_{1}^{2} \lambda_{2}-57 g_{2}^{2} \lambda_{2}+4 \lambda_{2}^{2}+\frac{35}{4} \lambda_{3}^{2}+70 \lambda_{4}^{2} \\
& +12 \lambda_{1} \lambda_{2}+4 \lambda_{2} \lambda_{6}+\frac{28}{3} \lambda_{2} \lambda_{7}+\frac{44}{3} \lambda_{2} \lambda_{8}+6 y_{t}^{2} \lambda_{2}+6 y_{b}^{2} \lambda_{2} 2 y_{\tau}^{2} \lambda_{2}, \\
& 16 \pi^{2} \frac{d \lambda_{3}}{d t}=\frac{18}{5} g_{1}^{2} g_{2}^{2}-\frac{9}{5} g_{1}^{2} \lambda_{3}-57 g_{2}^{2} \lambda_{3}+16 \lambda_{4}^{2}+4 \lambda_{1} \lambda_{3}+8 \lambda_{2} \lambda_{3} \\
& -\frac{124}{35} \lambda_{3} \lambda_{6}-\frac{44}{15} \lambda_{3} \lambda_{7}+\frac{220}{21} \lambda_{3} \lambda_{8}+6 y_{t}^{2} \lambda_{3}+6 y_{b}^{2} \lambda_{3}+2 y_{\tau}^{2} \lambda_{3}, \\
& 16 \pi^{2} \frac{d \lambda_{4}}{d t}=-\frac{9}{5} g_{1}^{2} \lambda_{4}-57 g_{2}^{2} \lambda_{4}+4 \lambda_{1} \lambda_{4}+8 \lambda_{2} \lambda_{4}+4 \lambda_{3} \lambda_{4} \\
& +4 \lambda_{4} \lambda_{6}+6 \lambda_{4} y_{t}^{2}+6 \lambda_{4} y_{b}^{2}+2 \lambda_{4} y_{\tau}^{2},
\end{aligned}
$$




$$
\begin{aligned}
& 16 \pi^{2} \frac{d \lambda_{6}}{d t}=\frac{27}{200} g_{1}^{4}+\frac{2697}{8} g_{2}^{4}-\frac{279}{20} g_{1}^{2} g_{2}^{2}-\frac{9}{5} g_{1}^{2} \lambda_{6}-105 g_{2}^{2} \lambda_{6}+2 \lambda_{2}^{2}-\frac{31}{8} \lambda_{3}^{2}+35 \lambda_{4}^{2} \\
& +\frac{8248}{1225} \lambda_{6}^{2}+\frac{2054}{675} \lambda_{7}^{2}+\frac{374}{1323} \lambda_{8}^{2}+\frac{88}{75} \lambda_{6} \lambda_{7}+\frac{2552}{147} \lambda_{6} \lambda_{8}+\frac{308}{27} \lambda_{7} \lambda_{8}, \\
& 16 \pi^{2} \frac{d \lambda_{7}}{d t}=\frac{27}{200} g_{1}^{4}+\frac{297}{8} g_{2}^{4}-\frac{99}{20} g_{1}^{2} g_{2}^{2}-\frac{9}{5} g_{1}^{2} \lambda_{7}-105 g_{2}^{2} \lambda_{7}+2 \lambda_{2}^{2}-\frac{11}{8} \lambda_{3}^{2} \\
& +\frac{44}{175} \lambda_{6}^{2}+\frac{21152}{2025} \lambda_{7}^{2}+\frac{1496}{567} \lambda_{8}^{2}+\frac{4108}{1575} \lambda_{6} \lambda_{7}+\frac{44}{9} \lambda_{6} \lambda_{8}+\frac{10868}{567} \lambda_{7} \lambda_{8}, \\
& 16 \pi^{2} \frac{d \lambda_{8}}{d t}=\frac{27}{200} g_{1}^{4}+\frac{2025}{8} g_{2}^{4}+\frac{45}{4} g_{1}^{2} g_{2}^{2}-\frac{9}{5} g_{1}^{2} \lambda_{8}-105 g_{2}^{2} \lambda_{8}+2 \lambda_{2}^{2}+\frac{25}{8} \lambda_{3}^{2} \\
& +\frac{116}{49} \lambda_{6}^{2}+\frac{494}{81} \lambda_{7}^{2}+\frac{98870}{3969} \lambda_{8}^{2}+\frac{28}{9} \lambda_{6} \lambda_{7}+\frac{68}{441} \lambda_{6} \lambda_{8}+\frac{272}{81} \lambda_{7} \lambda_{8} . \\
& \text { D.2. } n=8 \\
& 16 \pi^{2} \frac{d g_{1}}{d t}=\frac{9}{2} g_{1}^{3} \\
& 16 \pi^{2} \frac{d g_{2}}{d t}=\frac{65}{3} g_{2}^{3} \\
& 16 \pi^{2} \frac{d g_{3}}{d t}=-7 g_{3}^{3} \\
& 16 \pi^{2} \frac{d \mu_{h}^{2}}{d t}=-\frac{9}{10} g_{1}^{2} \mu_{h}^{2}-\frac{9}{2} g_{2}^{2} \mu_{h}^{2}+12 \lambda_{1} \mu_{h}^{2}+16 \lambda_{2} M^{2}+6 y_{t}^{2} \mu_{h}^{2}+6 \mu_{h}^{2} y_{b}^{2}+2 y_{\tau}^{2} \mu_{h}^{2}, \\
& 16 \pi^{2} \frac{d M^{2}}{d t}=-\frac{9}{10} g_{1}^{2} M^{2}-\frac{189}{2} g_{2}^{2} M^{2}+4 \lambda_{2} \mu_{h}^{2}+3 \lambda_{6} M^{2}+7 \lambda_{7} M^{2}+11 \lambda_{8} M^{2}+15 \lambda_{9} M^{2} \\
& 16 \pi^{2} \frac{d \lambda_{1}}{d t}=\frac{27}{200} g_{1}^{4}+\frac{9}{8} g_{2}^{4}+\frac{9}{20} g_{1}^{2} g_{2}^{2}-\frac{9}{5} g_{1}^{2} \lambda_{1}-9 g_{2}^{2} \lambda_{1}+24 \lambda_{1}^{2}+8 \lambda_{2}^{2}+\frac{21}{2} \lambda_{3}^{2} \\
& +84 \lambda_{4}^{2}+12 y_{t}^{2} \lambda_{1}+12 y_{b}^{2} \lambda_{1}+4 y_{\tau}^{2} \lambda_{1}-6 y_{t}^{4}-6 y_{b}^{4}-2 y_{\tau}^{4} \text {, }
\end{aligned}
$$




$$
\begin{gathered}
16 \pi^{2} \frac{d \lambda_{2}}{d t}=\frac{27}{100} g_{1}^{4}+\frac{189}{4} g_{2}^{4}-\frac{9}{5} g_{1}^{2} \lambda_{2}-99 g_{2}^{2} \lambda_{2}+4 \lambda_{2}^{2}+\frac{63}{4} \lambda_{3}^{2}+126 \lambda_{4}^{2}+12 \lambda_{1} \lambda_{2} \\
+3 \lambda_{2} \lambda_{6}+7 \lambda_{2} \lambda_{7}+11 \lambda_{2} \lambda_{8}+15 \lambda_{2} \lambda_{9}+6 y_{t}^{2} \lambda_{2}+6 y_{b}^{2} \lambda_{2}+2 y_{\tau}^{2} \lambda_{2} \\
16 \pi^{2} \frac{d \lambda_{3}}{d t}=\frac{18}{5} g_{1}^{2} g_{2}^{2}-\frac{9}{5} g_{1}^{2} \lambda_{3}-99 g_{2}^{2} \lambda_{3}+16 \lambda_{4}^{2}+4 \lambda_{1} \lambda_{3}+8 \lambda_{2} \lambda_{3}-\frac{59}{21} \lambda_{3} \lambda_{6} \\
-\frac{13}{3} \lambda_{3} \lambda_{7}-\frac{11}{21} \lambda_{3} \lambda_{8}+\frac{35}{3} \lambda_{3} \lambda_{9}+6 y_{t}^{2} \lambda_{3}+6 y_{b}^{2} \lambda_{3}+2 y_{\tau}^{2} \lambda_{3} \\
16 \pi^{2} \frac{d \lambda_{4}}{d t}=-\frac{9}{5} g_{1}^{2} \lambda_{4}-99 g_{2}^{2} \lambda_{4}+4 \lambda_{1} \lambda_{4}+8 \lambda_{2} \lambda_{4}+4 \lambda_{3} \lambda_{4} \\
+4 \lambda_{4} \lambda_{6}+6 y_{t}^{2} \lambda_{4}+6 y_{b}^{2} \lambda_{4}+2 y_{\tau}^{2} \lambda_{4}
\end{gathered}
$$

$$
\begin{aligned}
16 \pi^{2} \frac{d \lambda_{6}}{d t}= & \frac{27}{200} g_{1}^{4}+\frac{10089}{8} g_{2}^{4}-\frac{531}{20} g_{1}^{2} g_{2}^{2}-\frac{9}{5} g_{1}^{2} \lambda_{6}-189 g_{2}^{2} \lambda_{6}+2 \lambda_{2}^{2}-\frac{59}{8} \lambda_{3}^{2} \\
& +84 \lambda_{4}^{2}+\frac{10301}{1764} \lambda_{6}^{2}-\frac{155}{132} \lambda_{7}^{2}+\frac{25289}{2548} \lambda_{8}^{2}+\frac{115}{5148} \lambda_{9}^{2}+\frac{65}{18} \lambda_{6} \lambda_{7} \\
& -\frac{11}{882} \lambda_{6} \lambda_{8}-\frac{\lambda_{7} \lambda_{8}}{6}+\frac{265}{18} \lambda_{6} \lambda_{9}+\frac{2555}{198} \lambda_{7} \lambda_{9}+\frac{545}{234} \lambda_{8} \lambda_{9}, \\
16 \pi^{2} \frac{d \lambda_{7}}{d t}= & \frac{27}{200} g_{1}^{4}+\frac{4329}{8} g_{2}^{4}-\frac{351}{20} g_{1}^{2} g_{2}^{2}-\frac{9}{5} g_{1}^{2} \lambda_{7}-189 g_{2}^{2} \lambda_{7}+2 \lambda_{2}^{2} \\
& -\frac{39}{8} \lambda_{3}^{2}+\frac{65}{84} \lambda_{6}^{2}+\frac{10063}{1452} \lambda_{7}^{2}+\frac{1501}{1092} \lambda_{8}^{2}+\frac{4715}{18876} \lambda_{9}^{2}-\frac{155}{154} \lambda_{6} \lambda_{7} \\
& -\frac{\lambda_{6} \lambda_{8}}{14}+\frac{4735}{462} \lambda_{7} \lambda_{8}+\frac{365}{66} \lambda_{6} \lambda_{9}+\frac{3605}{242} \lambda_{7} \lambda_{9}+\frac{2595}{286} \lambda_{8} \lambda_{9},
\end{aligned}
$$

$$
\begin{aligned}
16 \pi^{2} \frac{d \lambda_{8}}{d t}= & \frac{27}{200} g_{1}^{4}+\frac{9}{8} g_{2}^{4}-\frac{27}{20} g_{1}^{2} g_{2}^{2}-\frac{9}{5} g_{1}^{2} \lambda_{8}-189 g_{2}^{2} \lambda_{8}+2 \lambda_{2}^{2}-\frac{3}{8} \lambda_{3}^{2}-\frac{\lambda_{6}^{2}}{588} \\
& +\frac{4735}{1452} \lambda_{7}^{2}+\frac{1009231}{99372} \lambda_{8}^{2}+\frac{621575}{245388} \lambda_{9}^{2}-\frac{\lambda_{6} \lambda_{7}}{22}+\frac{6897}{1274} \lambda_{6} \lambda_{8} \\
& +\frac{1501}{858} \lambda_{7} \lambda_{8}+\frac{545}{858} \lambda_{6} \lambda_{9}+\frac{18165}{3146} \lambda_{7} \lambda_{9}+\frac{68875}{3718} \lambda_{8} \lambda_{9},
\end{aligned}
$$




$$
\begin{aligned}
16 \pi^{2} \frac{d \lambda_{9}}{d t}= & \frac{27}{200} g_{1}^{4}+\frac{7497}{8} g_{2}^{4}+\frac{441}{20} g_{1}^{2} g_{2}^{2}-\frac{9}{5} g_{1}^{2} \lambda_{9}-189 g_{2}^{2} \lambda_{9}+2 \lambda_{2}^{2}+\frac{49}{8} \lambda_{3}^{2} \\
& +\frac{53}{36} \lambda_{6}^{2}+\frac{5047}{1452} \lambda_{7}^{2}+\frac{13775}{2028} \lambda_{8}^{2}+\frac{18419861}{736164} \lambda_{9}^{2}+\frac{511}{198} \lambda_{6} \lambda_{7} \\
& +\frac{109}{234} \lambda_{6} \lambda_{8}+\frac{1211}{286} \lambda_{7} \lambda_{8}+\frac{23}{2574} \lambda_{6} \lambda_{9}+\frac{6601}{28314} \lambda_{7} \lambda_{9}+\frac{124315}{33462} \lambda_{8} \lambda_{9} .
\end{aligned}
$$




\section{APPENDIX E \\ $U(1)$-symmetric models}

The models described in Chapter 3 are not the only possible large electroweak scalar multiplet models. If we make the symmetry requirement more stringent, then the $\lambda_{4}$ term in the potential will be excluded. In this appendix, we will give a brief summary of our results as presented in Ref. [5. A $U(1)$-symmetric scalar potential is given by

$$
\begin{array}{r}
V(\Phi, \Sigma)=m^{2} \Phi^{\dagger} \Phi+M^{2} \Sigma^{\dagger} \Sigma \\
+\lambda_{1}\left(\Phi^{\dagger} \Phi\right)^{2}+\lambda_{2} \Phi^{\dagger} \Phi \Sigma^{\dagger} \Sigma \\
+\lambda_{3} \Phi^{\dagger} T_{\Phi}^{a} \Phi \Sigma^{\dagger} T_{\Sigma}^{a} \Sigma+\mathcal{O}\left(\Sigma^{4}\right)
\end{array}
$$

where $\Phi$ is the usual SM Higgs doublet, $\Sigma=\left(\sigma^{+(n-1)}, \ldots, \sigma^{0}\right)^{\mathrm{T}}$ is the large multiplet (taking $Y_{\Sigma}=2 T$ so that the lightest member can be made neutral), and $T_{\Phi}^{a}$ and $T_{\Sigma}^{a}$ are the $S U(2)_{L}$ generators. In these models, we may have $n=5,6,7$, or 8 . In the case of $n=5$, however, there is a dimension-five Planck-suppressed interaction with the SM Higgs doublet,

$$
\mathcal{L} \supset \frac{1}{m_{\mathrm{Pl}} .} \Phi \Phi \Phi \Phi \Sigma^{\dagger}+\text { h.c. },
$$

which induces a mixing of the SM Higgs into the neutral component of $\Sigma$,

$$
\sigma^{\prime}=\sigma^{0, r}-\epsilon \phi^{0, r} \quad, \quad \epsilon \sim \frac{v^{3}}{\left(m_{\sigma^{0}}^{2}-m_{h}^{2}\right) m_{\mathrm{Pl}} .} .
$$

The neutral member of $\Sigma$ can then decay via its Higgs component. Assuming that $\sigma^{0}$ is the lightest state, then its lifetime ranges from a few days to a few years in the mass range $m_{\sigma^{0}} \sim 100-1000 \mathrm{GeV}$. These decays mean that the potential DM candidate 


\begin{tabular}{c|cc}
\hline \hline$n$ & $\left|\lambda_{2}\right|^{\text {MAX }}$ & $\left|\lambda_{3}\right|^{\text {MAX }}$ \\
\hline 6 & 6.49 & 8.17 \\
7 & 5.01 & 6.11 \\
8 & 2.17 & 4.41 \\
\hline \hline
\end{tabular}

TABLE E.1. Upper limits on $\left|\lambda_{2}\right|$ and $\left|\lambda_{3}\right|$ from perturbative unitarity for the $U(1)$-symmetric models with $n=6,7$, and 8 .

in the $n=5$ model will decay away well before the recombination temperature, $T_{\text {recomb. }}$, and thus not constitute any of the DM present in the Universe today. In the remainder, therefore, we consider only the $n=6,7$, and 8 models. We will show that a combination of the electroweak precision observables $(S T U)$, the observed limits on the rate for decays of a Higgs boson to two photons, and the direct-detection limits will exclude these models entirely.

The mass of particle $\sigma^{Q}$ with charge $Q=T^{3}+Y / 2 \geq 0$ is given by

$$
m_{\sigma^{Q}}^{2}=M^{2}+\frac{1}{2} v^{2}\left[\lambda_{2}-\frac{1}{2}\left(Q-\frac{n-1}{2}\right) \lambda_{3}\right]=M^{2}+\frac{1}{2} v^{2} \Lambda_{Q}=m_{\sigma^{0}}^{2}-\frac{1}{4} v^{2} Q \lambda_{3} .
$$

We want $\sigma^{0}$ to be the DM, which leads to the constraint $\lambda_{3}<0$.

We can use perturbative unitarity to place upper bounds on $\lambda_{2,3}$, shown in Table E.1. The contributions to the electroweak precision observables, $S T U$, are given 
by

$$
\begin{aligned}
& S=-\frac{Y}{6 \pi} \sum_{Q=0}^{n-1}\left(Q-\frac{n-1}{2}\right) \log \left[m_{\sigma^{Q}}^{2}\right], \\
& T=\frac{1}{4 \pi m_{Z}^{2} s_{W}^{2} c_{W}^{2}}\left\{\sum_{Q=0}^{n-1} m_{\sigma^{Q}}^{2}\left[(n-1)\left(Q+\frac{1}{2}\right)-Q^{2}\right] \log \left[m_{\sigma^{Q}}^{2}\right]\right. \\
& \left.\quad-\sum_{Q=0}^{n-2}(Q+1)(n-1-Q) f_{2}\left[m_{\sigma^{Q}}, m_{\sigma^{Q+1}}\right]\right\}, \\
& U=\frac{1}{\pi}\left\{\sum_{Q=0}^{n-2}(Q+1)(n-1-Q) f_{1}\left[m_{\sigma^{Q}}, m_{\sigma^{Q+1}}\right]\right. \\
& \left.\quad-\frac{1}{3} \sum_{Q=0}^{n-1}\left(Q-\frac{n-1}{2}\right) \log \left[m_{\sigma^{Q}}^{2}\right]\right\},
\end{aligned}
$$

where

$$
\begin{aligned}
& f_{1}\left(m_{1}, m_{2}\right)=\int_{0}^{1} d x x(1-x) \log \left[x m_{1}^{2}+(1-x) m_{2}^{2}\right] \\
& f_{2}\left(m_{1}, m_{2}\right)=\int_{0}^{1} d x\left[x m_{1}^{2}+(1-x) m_{2}^{2}\right] \log \left[x m_{1}^{2}+(1-x) m_{2}^{2}\right] .
\end{aligned}
$$

We constrain these contributions to the oblique parameters using a $\chi^{2}$ variable including correlations in the measured values of $S T U$,

$$
\chi^{2}=\sum_{i, j=S, T, U} \frac{\left(\mathcal{O}_{i}-\mathcal{O}_{i}^{\text {exp }}\right)\left(\mathcal{O}_{j}-\mathcal{O}_{j}^{\text {exp }}\right)}{\left[\sigma^{2}\right]_{i j}},
$$

where $\left[\sigma^{2}\right]_{i j}=\Delta \mathcal{O}_{i} \Delta \mathcal{O}_{j} \rho_{i j}$ are the uncertainties and $\rho_{i j}$ are the correlations between the observables (note $\rho_{i i}=1$ ), as given in Table 5.2.

Using the experimental values for $S T U$ and the unitarity bounds on $\lambda_{2,3}$ we can, for example, constrain the mass splitting between the first charged state and the 

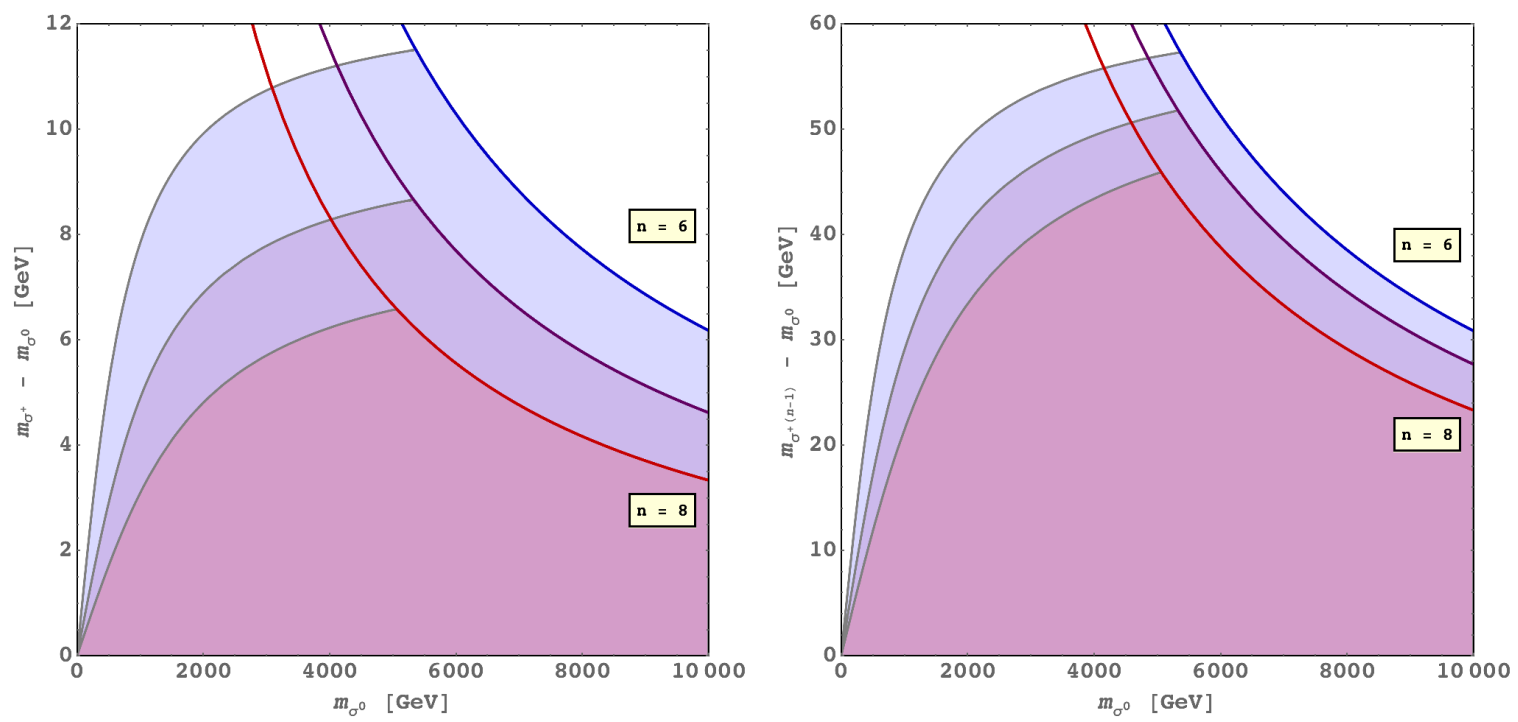

Figure E.1. Mass splitting in the $U(1)$-symmetric models. The plot on the left shows the splitting between $\sigma^{+}$and $\sigma^{0}$, while the plot on the right shows the splitting between $\sigma^{n-1}$ and $\sigma^{0}$. The shaded region corresponds to the splitting allowed by both unitarity and $S T U$ constraints. The solid curve is the unitarity bound for each $n$.

neutral state. We plot this result in Figure E.1 for $n=6,7$, and 8. The shaded region represents the mass splitting allowed by both unitarity and $S T U$.

Next, we use the rate of Higgs decays to two photons. The width of this decay is given by 169

$$
\Gamma(h \rightarrow \gamma \gamma)=\frac{\alpha_{\mathrm{EM}}^{2} g^{2}}{1024 \pi^{3}} \frac{m_{h}^{3}}{m_{W}^{2}}\left|\sum_{i} N_{c i} Q_{i}^{2} F_{i}(\tau)\right|^{2},
$$

where $i$ runs over charged particles of spin $1,1 / 2$, and $0, Q$ is the electric charge in units of $e, N_{c i}$ is the color multiplicity and the functions $F_{i}(\tau)$ depend on the particle's spin,

$$
\begin{aligned}
F_{1} & =2+3 \tau+3 \tau(2-\tau) f(\tau), \\
F_{1 / 2} & =-2 \tau[1+(1-\tau) f(\tau)], \\
F_{0} & =\beta \tau[1-\tau f(\tau)] .
\end{aligned}
$$


Here $\tau=4 m_{i}^{2} / m_{h}^{2}$, and the function $f(\tau)$ is given by

$$
f(\tau)= \begin{cases}{\left[\arcsin \left(\sqrt{\frac{1}{\tau}}\right)\right]^{2}} & \tau \geq 1, \\ -\frac{1}{4}\left[\ln \left(\frac{\eta_{+}}{\eta_{-}}-i \pi\right)\right]^{2} & \tau<1,\end{cases}
$$

where we have defined $\eta_{ \pm}=1 \pm \sqrt{1-\tau}$. For the scalars, the coupling to the Higgs is parameterized by

$$
\beta_{i}=\frac{v^{2} \Lambda_{Q} / 2}{M^{2}+v^{2} \Lambda_{Q} / 2}
$$

where $\Lambda_{n}$ was defined in Equation (E.4),

Since there is no splitting between the real and imaginary components of $\sigma^{0}$, the coupling to a $Z$ does not change the species. Thus, it will contribute to the direct detection cross section. The total scattering cross section will be

$$
\sigma_{\mathrm{SI}}^{\sigma^{0}}=\sigma_{Z}^{\sigma^{0}}+\sigma_{h}^{\sigma^{0}}
$$

where

$$
\begin{aligned}
\sigma_{Z}^{\sigma^{0}} & =\frac{\left(f_{N}^{V}\right)^{2}(n-1)^{2}}{\pi} \frac{m_{\sigma^{0}}^{2}}{v^{2} m_{Z}^{2}} \frac{m_{N}^{2}}{\left(m_{N}+m_{\sigma^{0}}\right)^{2}}, \\
\sigma_{h}^{\sigma^{0}} & =\frac{\left(f_{N}^{h}\right)^{2} \Lambda_{0}^{2}}{4 \pi} \frac{v^{2}}{m_{h}^{4}} \frac{m_{N}^{2}}{\left(m_{N}+m_{\sigma^{0}}\right)^{2}},
\end{aligned}
$$

and the effective boson-nucleon couplings, $f_{N}^{V, h}$, are given by [217, 209]

$$
\begin{aligned}
f_{p}^{V} & =\frac{2 m_{Z}}{v}\left(\frac{1}{4}-s_{W}^{2}\right), & f_{n}^{V} & =\frac{2 m_{Z}}{v}\left(-\frac{1}{4}\right), \\
f_{p}^{h} & =\frac{m_{p}}{v}(0.350 \pm 0.048) & , & f_{n}^{h}=\frac{m_{n}}{v}(0.353 \pm 0.049) .
\end{aligned}
$$

Because dark matter particles moving in the galactic halo have de Broglie wavelengths that are large compared to the size of a nucleus, the amplitudes for scattering off each 
nucleon add coherently. This can be accounted for by replacing $f_{N}^{V}$ and $f_{N}^{h}$ with

$$
\begin{aligned}
& \left(f_{N}^{V}\right)^{2} \rightarrow\left(\overline{f_{N}^{V}}\right)^{2}=\left[\frac{Z f_{p}^{V}+(A-Z) f_{N}^{V}}{A}\right]^{2} \\
& \left(f_{N}^{h}\right)^{2} \rightarrow\left(\overline{f_{N}^{h}}\right)^{2}=\left[\frac{Z f_{p}^{h}+(A-Z) f_{N}^{h}}{A}\right]^{2} \approx\left(f_{p}^{h}\right)^{2} \approx\left(f_{n}^{h}\right)^{2}
\end{aligned}
$$

A weighted average is taken over the natural abundances of the relevant isotopes. We take the natural abundances from Ref. [218. In this case, we are comparing to XENON100 [219], so $Z=54$ and $A$ ranges from 124 to 136 .

The fraction of the DM density that can be attributed to $\sigma^{0}$ is bounded from above by the experimental bounds from direct detection experiments such as XENON100. We can thus exclude the region defined by

$$
\frac{\Omega_{\sigma}}{\Omega_{\mathrm{DM}}} \geq \frac{\sigma_{\mathrm{SI}}^{\mathrm{Xe} 100}}{\sigma_{\mathrm{SI}}^{\sigma^{0}}}
$$

The DM fraction of $\sigma^{0}$ may be determined using

$$
\frac{\Omega_{\sigma}}{\Omega_{\mathrm{DM}}}=\frac{\langle\sigma \beta\rangle_{\mathrm{STD}}}{\frac{1}{2}\left\langle\sigma \beta\left(\sigma^{0 *} \sigma^{0} \rightarrow W^{+} W^{-}, Z Z, h h, f \bar{f}\right)\right\rangle}
$$

The annihilation cross sections to gauge boson two-body final states are given in the $\beta \rightarrow 0$ limit by,

$$
\begin{array}{r}
\sigma \beta\left(\sigma^{0 *} \sigma^{0} \rightarrow W^{+} W^{-}\right)=\frac{m_{W}^{4}}{8 \pi v} \sqrt{1}-\frac{m_{W}^{2}}{m_{\sigma^{0}}^{2}}\left[\frac{A_{W}^{2}}{m_{\sigma^{0}}^{2}}\left(3-4 \frac{m_{\sigma^{0}}^{2}}{m_{W}^{2}}+4 \frac{m_{\sigma^{0}}^{4}}{m_{W}^{4}}\right)\right. \\
+2 A_{W} B_{W}\left(1-3 \frac{m_{\sigma^{0}}^{2}}{m_{W}^{2}}+2 \frac{m_{\sigma^{0}}^{4}}{m_{W}^{4}}\right) \\
\left.+B_{W}^{2} m_{\sigma^{0}}^{2}\left(1-\frac{m_{\sigma^{0}}^{2}}{m_{W}^{2}}\right)^{2}\right]
\end{array}
$$


and

$$
\begin{array}{r}
\sigma \beta\left(\sigma^{0 *} \sigma^{0} \rightarrow Z Z\right)=\frac{m_{Z}^{4}}{16 \pi v} \sqrt{1-} \frac{m_{Z}^{2}}{m_{\sigma^{0}}^{2}}\left[\frac{A_{Z}^{2}}{m_{\sigma^{0}}^{2}}\left(3-4 \frac{m_{\sigma^{0}}^{2}}{m_{Z}^{2}}+4 \frac{m_{\sigma^{0}}^{4}}{m_{Z}^{4}}\right)\right. \\
+2 A_{Z} B_{Z}\left(1-3 \frac{m_{\sigma^{0}}^{2}}{m_{Z}^{2}}+2 \frac{m_{\sigma^{0}}^{4}}{m_{Z}^{4}}\right) \\
\left.+B_{Z}^{2} m_{\sigma^{0}}^{2}\left(1-\frac{m_{\sigma^{0}}^{2}}{m_{Z}^{2}}\right)^{2}\right]
\end{array}
$$

where the coefficients are given by

$$
\begin{aligned}
A_{Z} & =(n-1)^{2}+\frac{\Lambda_{0} v^{2}}{4 m_{\sigma^{0}}^{2}-m_{h}^{2}} \\
B_{Z} & =-\frac{4(n-1)^{2}}{2 m_{\sigma^{0}}^{2}-m_{Z}^{2}} \\
A_{W} & =(n-1)+\frac{\Lambda_{0} v^{2}}{4 m_{\sigma^{0}}^{2}-m_{h}^{2}}, \\
B_{W} & =\frac{4(n-1)}{m_{W}^{2}-m_{\sigma^{0}}^{2}-m_{\sigma^{+}}^{2}} .
\end{aligned}
$$

The annihilation cross sections to Higgs and fermion final states are given by

$$
\sigma \beta\left(\sigma^{0 *} \sigma^{0} \rightarrow h h\right)=\frac{\Lambda_{0}^{2}}{64 \pi m_{\sigma^{0}}^{2}} \sqrt{1-\frac{m_{h}^{2}}{m_{\sigma^{0}}^{2}}}\left[1+\frac{3 m_{h}^{2}}{4 m_{\sigma^{0}}^{2}-m_{h}^{2}}-\frac{2 v \Lambda_{0}}{2 m_{\sigma^{0}}^{2}-m_{h}^{2}}\right]^{2}
$$

and

$$
\sigma \beta\left(\sigma^{0 *} \sigma^{0} \rightarrow f \bar{f}\right)=\frac{N_{c}}{4 \pi}\left[1-\frac{m_{f}^{2}}{m_{\sigma^{0}}^{2}}\right]^{\frac{3}{2}} \frac{m_{f}^{2} \Lambda_{0}^{2}}{\left(4 m_{\sigma^{0}}^{2}-m_{h}^{2}\right)^{2}},
$$

where $N_{c}$ is the number of colours of the final-state fermions, and recall that the Higgs coupling is given by

$$
\Lambda_{0}=\lambda_{2}-\frac{n-1}{4} \lambda_{3}
$$



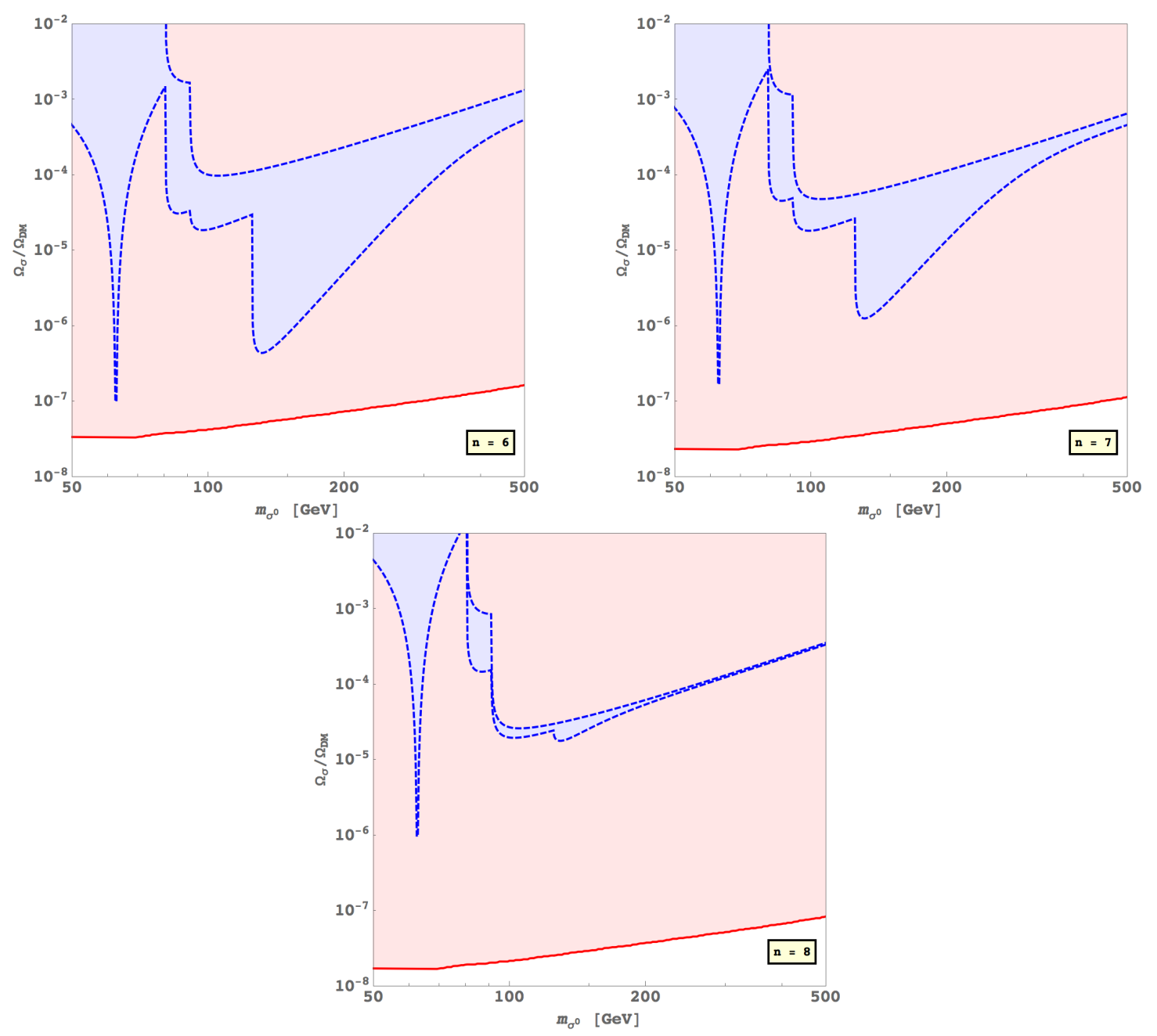

FiguRE E.2. DM fraction and direct detection bound for $U(1)$ symmetric models. The left panel is $n=6$, the middle panel is $n=7$, and the right panel is $n=8$. The shaded blue region bounded by the dashed blue lines is the DM fraction from relic density calculations allowed by both unitarity and $S T U$. The red curve is the upper-bound on direct detection cross section from the XENON100 experiment. Everything above the red curve is excluded.

We may then plot both Equations (E.16) and (E.17) together on the same plot, as in Figure E.2. The pink regions show the DM fraction that is excluded by Equation (E.16), while the blue regions bounded by the dashed blue lines show the relic 
abundance from Equation (E.17) for values of $\lambda_{2,3}$ within the unitarity and $S T U$ bounds. Notice that the dashed blue region all sits entirely within the excluded regions. This indicates that these models should already have been observed in direct detection experiments over the entire parameter space, and are hence ruled out.

The most interesting feature in the annihilation cross section below the $W W$ threshold is the Higgs pole at $m_{\sigma^{0}}=m_{h} / 2$. The possibility that this resonant annihilation may suppress the $\sigma^{0}$ relic density enough to evade the direct detection constraints is shown in Figure E.3. Unfortunately, this possibility is excluded by a combination of constraints from the oblique parameters and the observed rate for $p p \rightarrow h \rightarrow \gamma \gamma$ from the LHC [220, 221].

The $\sigma^{0} \sigma^{0 *} \rightarrow h \rightarrow b \bar{b}$ annihilation cross section near the Higgs resonance is obtained from Equation (E.22) by making the replacement in the denominator,

$$
\left(4 m_{\sigma^{0}}^{2}-m_{h}^{2}\right)^{2} \rightarrow\left(4 m_{\sigma^{0}}^{2}-m_{h}^{2}\right)^{2}+m_{h}^{2} \Gamma_{h}^{2}
$$

Here $\Gamma_{h}$ is the total decay width of the SM Higgs boson; the contribution from $b \bar{b}$ final states is given at tree level by [169],

$$
\Gamma(h \rightarrow b \bar{b})=\frac{N_{c} m_{b}^{2} m_{h}}{8 \pi v^{2}}\left(1-\frac{4 m_{b}^{2}}{m_{h}^{2}}\right)^{3 / 2},
$$

where $N_{c}=3$ is the number of colours.

Note that we can capture the effects of higher-order corrections and additional final states on $\sigma \beta\left(\chi^{0} \chi^{0 *} \rightarrow h \rightarrow\right.$ any $)$ near the Higgs resonance simply by choosing the value of $m_{b}$ to yield the correct total SM Higgs width, $\Gamma_{h}=4.07 \pm 0.16 \mathrm{MeV}[\mathbf{2 2 2}]$ for $m_{h}=125 \mathrm{GeV}$. We obtain this width from the tree-level formula above when $m_{b}=4.08 \mathrm{GeV}$. Using this value of $m_{b}$, we compute $\Omega_{\sigma} / \Omega_{\mathrm{DM}}$ in the vicinity of the Higgs resonance using the tree-level $\sigma^{0} \sigma^{0 *} \rightarrow b \bar{b}$ cross section and $h \rightarrow b \bar{b}$ partial 

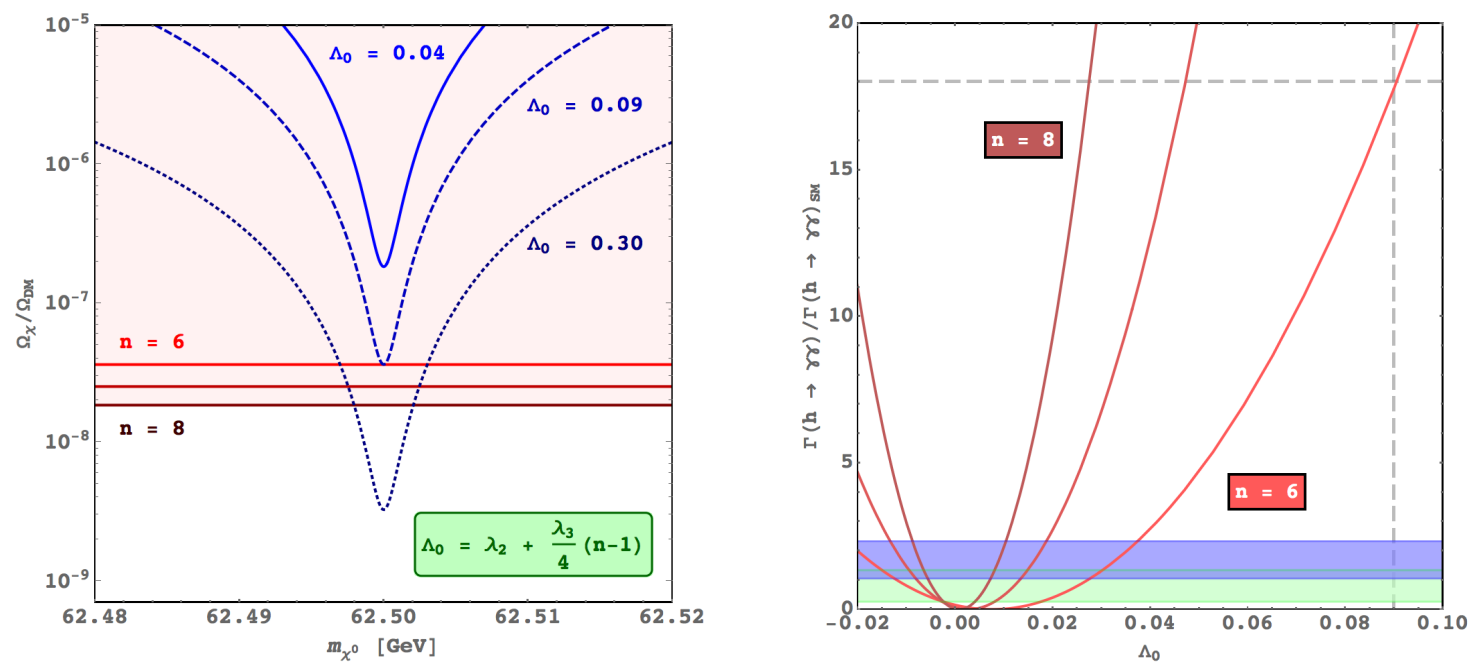

Figure E.3. The plot on the left shows a close-up of the resonance region from Figure E.2 with values of $\Lambda_{0}$ labelled on each blue curve. The XENON100 bound is shown in red for each $n$. The plot on the right shows the experimental bounds on $\Gamma(h \rightarrow \gamma \gamma) / \Gamma(h \rightarrow \gamma \gamma)_{\mathrm{SM}}$ from ATLAS (shaded blue) and CMS (shaded green), along with curves for the $U(1)$-symmetric models (red). The required $\Lambda_{0} \approx 0.09$ to evade the direct-detection limits requires a rate $\Gamma(h \rightarrow \gamma \gamma) / \Gamma(h \rightarrow \gamma \gamma)_{\mathrm{SM}} \approx 18$, which is far excluded by LHC data.

width. The result is shown in Figure E.3 for various values of $\Lambda_{0}^{1}$. From these plots, we see that the value of $\Lambda_{0}$ required to evade the direct detection limits, $\Lambda_{0} \approx 0.09$, gives a rate $\Gamma(h \rightarrow \gamma \gamma) / \Gamma(h \rightarrow \gamma \gamma)_{\mathrm{SM}} \approx 18$, while the measured rate is near to one.

In this appendix, we have shown that models with a $U(1)$-symmetric scalar potential and $n=6,7$, and 8 are excluded by the incompatibility of the standard thermal freeze-out relic abundance with the dark matter direct detection cross section limit. This incompatibility may be bypassed if the requirement of $U(1)$ symmetry is relaxed to, for example, a $Z_{2}$-symmetric scalar potential as in the main text of this thesis. For these models, the $Z$ boson exchange contribution to the direct detection cross section will go away.

\footnotetext{
${ }^{1}$ When specified in terms of $\Lambda_{0}, \sigma \beta\left(\sigma^{0} \sigma^{0 *} \rightarrow b \bar{b}\right)$ is independent of $n$.
} 


\section{References}

[1] K. Hally, H. E. Logan, and T. Pilkington, "Constraints on large scalar multiplets from perturbative unitarity," Phys. Rev. D85 (2012) 095017, arXiv:1202.5073 [hep-ph].

[2] K. Earl, K. Hartling, H. E. Logan, and T. Pilkington, "Two viable large scalar multiplet models with a $\mathrm{Z}_{2}$ symmetry," Phys. Rev. D90 (2014) no. 5, 055029, arXiv:1311.3656 [hep-ph]. [Erratum: Phys. Rev. D92, no.3, 039902 (2015)].

[3] H. E. Logan, T. A. Martin, and T. Pilkington, "LHC constraints on large scalar multiplet models with a $\mathrm{Z}_{2}$ symmetry," Phys. Rev. D92 (2015) no. 5, 055028, arXiv:1507.01887 [hep-ph].

[4] M.-J. Harris, H. E. Logan, and T. Pilkington, "The effect of co-annihilation and Sommerfeld enhancement on the high-mass region of large multiplet models,". in preparation.

[5] K. Earl, K. Hartling, H. E. Logan, and T. Pilkington, "Constraining models with a large scalar multiplet," Phys. Rev. D88 (2013) 015002, arXiv:1303.1244 [hep-ph].

[6] Particle Data Group Collaboration, K. A. Olive et al., "Review of Particle Physics," Chin. Phys. C38 (2014) 090001

[7] D. Griffiths, Introduction to elementary particles. Wiley-VCH, Weinheim, Germany, 2008.

[8] LHCb Collaboration, R. Aaij et al., "Determination of the X(3872) meson quantum numbers," Phys. Rev. Lett. 110 (2013) 222001, arXiv:1302.6269 [hep-ex]

[9] LHCb Collaboration, R. Aaij et al., "Observation of $J / \psi p$ Resonances Consistent with Pentaquark States in $\Lambda_{b}^{0} \rightarrow J / \psi K^{-} p$ Decays," Phys. Rev. Lett. 115 (2015) 072001, arXiv: 1507.03414 [hep-ex]

[10] ATLAS Collaboration, G. Aad et al., "Observation of a new particle in the search for the Standard Model Higgs boson with the ATLAS detector at the LHC," Phys. Lett. B716 (2012) 1-29, arXiv:1207.7214 [hep-ex]. 
[11] CMS Collaboration, S. Chatrchyan et al., "Observation of a new boson at a mass of 125 GeV with the CMS experiment at the LHC," Phys. Lett. B716 (2012) 30-61, arXiv:1207.7235 [hep-ex].

[12] J. L. Hewett, "The Standard model and why we believe it," in Supersymmetry, supergravity and supercolliders. Proceedings, Theoretical Advanced Study Institute in elementary particle physics, TASI'97, Boulder, USA, June 2-27, 1997, pp. 3-83. 1997. arXiv:hep-ph/9810316 [hep-ph]

[13] T. Gehrmann, "Introduction to QCD," in Theoretical Advanced Study Institute in Elementary Particle Physics: Journeys Through the Precision Frontier: Amplitudes for Colliders (TASI 2014) Boulder, Colorado, June 2-27, 2014, L. Dixon and F. Petriello, eds., pp. 1-38. World Scientific, 2015.

[14] H. E. Logan, "TASI 2013 lectures on Higgs physics within and beyond the Standard Model," arXiv:1406.1786 [hep-ph].

[15] DELPHI, OPAL, LEP Electroweak, ALEPH, L3 Collaboration, S. Schael et al., "Electroweak Measurements in Electron-Positron Collisions at W-Boson-Pair Energies at LEP," Phys. Rept. 532 (2013) 119-244, arXiv:1302.3415 [hep-ex].

[16] ATLAS Standard Model physics group, Historic versions of ATLAS_a_SMSummary_TotalXsect from the ATLAS Standard Model physics group. ATLAS Standard Model physics group, 2016. https://atlas.web.cern.ch/Atlas/GROUPS/ PHYSICS/CombinedSummaryPlots/SM/ATLAS_a_SMSummary_TotalXsect/history.html.

[17] J. B. Hartle, An introduction to Einstein's general relativity. Addison-Wesley, San Francisco, USA, 2003.

[18] J. Oort, "The force exerted by the stellar system in the direction perpendicular to the galactic plane and some related problems," Bulletin of the Astronomical Institutes of the Netherlands 6 (1932) 249-287.

[19] F. Zwicky, "Die Rotverschiebung von extragalaktischen Nebeln," Helv. Phys. Acta 6 (1933) $110-127$.

[20] F. Zwicky, "On the Masses of Nebulae and of Clusters of Nebulae," Astrophys. J. 86 (1937) $217-246$ 
[21] V. C. Rubin and W. K. Ford, Jr., "Rotation of the Andromeda Nebula from a Spectroscopic Survey of Emission Regions," Astrophys. J. 159 (1970) 379-403.

[22] V. C. Rubin, N. Thonnard, and W. K. Ford, Jr., "Rotational properties of 21 SC galaxies with a large range of luminosities and radii, from NGC $4605 \mathrm{R}=4 \mathrm{kpc}$ to UGC $2885 \mathrm{R}=$ 122 kpc," Astrophys. J. 238 (1980) 471.

[23] A. Einstein, "The Field Equations of Gravitation," Sitzungsber. Preuss. Akad. Wiss. Berlin (Math. Phys.) 1915 (1915) 844-847.

[24] D. Kennefick, "Not only because of theory: Dyson, Eddington and the competing myths of the 1919 eclipse expedition," arXiv:0709.0685 [physics.hist-ph].

[25] Planck Collaboration, P. A. R. Ade et al., "Planck 2015 results. XIII. Cosmological parameters," arXiv:1502.01589 [astro-ph.C0].

[26] M. Milgrom, "A Modification of the Newtonian dynamics as a possible alternative to the hidden mass hypothesis," Astrophys. J. 270 (1983) 365-370.

[27] J. D. Bekenstein, "Relativistic gravitation theory for the MOND paradigm," Phys. Rev. D70 (2004) 083509, arXiv:astro-ph/0403694 [astro-ph]. [Erratum: Phys.

Rev.D71,069901(2005)].

[28] S. S. McGaugh, "A tale of two paradigms: the mutual incommensurability of $\Lambda \mathrm{CDM}$ and MOND," Can. J. Phys. 93 (2015) no. 2, 250-259, arXiv:1404.7525 [astro-ph.C0].

[29] T. D. Brandt, "Constraints on MACHO Dark Matter from Compact Stellar Systems in Ultra-Faint Dwarf Galaxies," Astrophys. J. 824 (2016) no. 2, L31, arXiv: 1605.03665 [astro-ph.GA].

[30] M. A. Monroy-Rodríguez and C. Allen, "The end of the MACHO era- revisited: new limits on MACHO masses from halo wide binaries," Astrophys. J. 790 (2014) no. 2, 159 arXiv:1406.5169 [astro-ph.GA]

[31] R. Holman, G. Lazarides, and Q. Shafi, "Axions and the Dark Matter of the Universe," Phys. Rev. D27 (1983) 995

[32] J. Preskill, M. B. Wise, and F. Wilczek, "Cosmology of the Invisible Axion," Phys. Lett. B120 (1983) 127-132

[33] D. J. E. Marsh, "Axion Cosmology," arXiv:1510.07633 [astro-ph.CO]. 
[34] D. E. Kaplan, M. A. Luty, and K. M. Zurek, "Asymmetric Dark Matter," Phys. Rev. D79 (2009) 115016, arXiv:0901.4117 [hep-ph].

[35] K. Petraki and R. R. Volkas, "Review of asymmetric dark matter," Int. J. Mod. Phys. A28 (2013) 1330028, arXiv:1305.4939 [hep-ph].

[36] K. M. Zurek, "Asymmetric Dark Matter: Theories, Signatures, and Constraints," Phys. Rept. 537 (2014) 91-121, arXiv:1308.0338 [hep-ph].

[37] K. Benakli, J. R. Ellis, and D. V. Nanopoulos, "Natural candidates for superheavy dark matter in string and M theory," Phys. Rev. D59 (1999) 047301, arXiv:hep-ph/9803333 [hep-ph].

[38] H.-C. Cheng, J. L. Feng, and K. T. Matchev, "Kaluza-Klein dark matter," Phys. Rev. Lett. 89 (2002) 211301, arXiv:hep-ph/0207125 [hep-ph].

[39] G. Servant and T. M. P. Tait, "Is the lightest Kaluza-Klein particle a viable dark matter candidate?," Nucl. Phys. B650 (2003) 391-419, arXiv:hep-ph/0206071 [hep-ph].

[40] K. Agashe and G. Servant, "Baryon number in warped GUTs: Model building and (dark matter related) phenomenology," JCAP 0502 (2005) 002, arXiv: hep-ph/0411254 [hep-ph].

[41] L. Ackerman, M. R. Buckley, S. M. Carroll, and M. Kamionkowski, "Dark Matter and Dark Radiation," Phys. Rev. D79 (2009) 023519, arXiv:0810.5126 [hep-ph].

[42] J. L. Feng, H. Tu, and H.-B. Yu, "Thermal Relics in Hidden Sectors," JCAP 0810 (2008) 043, arXiv:0808.2318 [hep-ph].

[43] J. R. Ellis, J. S. Hagelin, D. V. Nanopoulos, K. A. Olive, and M. Srednicki, "Supersymmetric Relics from the Big Bang," Nucl. Phys. B238 (1984) 453-476.

[44] G. Steigman and M. S. Turner, "Cosmological Constraints on the Properties of Weakly Interacting Massive Particles," Nucl. Phys. B253 (1985) 375-386.

[45] J. L. Feng, "Dark Matter Candidates from Particle Physics and Methods of Detection," Ann. Rev. Astron. Astrophys. 48 (2010) 495-545, arXiv:1003.0904 [astro-ph.CO].

[46] R. D. Peccei and H. R. Quinn, "CP Conservation in the Presence of Instantons," Phys. Rev. Lett. 38 (1977) 1440-1443. 
[47] R. D. Peccei and H. R. Quinn, "Constraints Imposed by CP Conservation in the Presence of Instantons," Phys. Rev. D16 (1977) 1791-1797

[48] J. McDonald, "Baryomorphosis: Relating the Baryon Asymmetry to the 'WIMP Miracle'," Phys. Rev. D83 (2011) 083509, arXiv:1009.3227 [hep-ph].

[49] R. Rattazzi, "Cargese lectures on extra-dimensions," in Particle physics and cosmology: The interface. Proceedings, NATO Advanced Study Institute, School, Cargese, France, August 4-16, 2003, pp. 461-517. 2003. arXiv:hep-ph/0607055 [hep-ph]

[50] C. Csaki, "TASI lectures on extra dimensions and branes," in From fields to strings: Circumnavigating theoretical physics. Ian Kogan memorial collection (3 volume set), pp. 605-698. 2004. arXiv:hep-ph/0404096 [hep-ph].

[51] H.-C. Cheng, "Introduction to Extra Dimensions," in Physics of the large and the small, TASI 09, proceedings of the Theoretical Advanced Study Institute in Elementary Particle Physics, Boulder, Colorado, USA, 1-26 June 2009, pp. 125-162. 2011. arXiv:1003.1162 [hep-ph].

[52] H. M. Hodges, "Shadow baryons as the dark matter," in Potsdam 1991, Proceedings, Relativistic astrophysics and cosmology* 52-56. 1991.

[53] H. M. Hodges, "Mirror baryons as the dark matter," Phys. Rev. D47 (1993) 456-459.

[54] R. N. Mohapatra and V. L. Teplitz, "Mirror dark matter," in Proceedings, 7th International Symposium on Particles, strings and cosmology (PASCOS 1999). 2000. arXiv:astro-ph/0004046 [astro-ph].

[55] G. Jungman, M. Kamionkowski, and K. Griest, "Supersymmetric dark matter," Phys. Rept. 267 (1996) 195-373, arXiv: hep-ph/9506380 [hep-ph].

[56] M. Cirelli, N. Fornengo, and A. Strumia, "Minimal dark matter," Nucl. Phys. B753 (2006) 178-194, arXiv:hep-ph/0512090 [hep-ph].

[57] M. Cirelli, A. Strumia, and M. Tamburini, "Cosmology and Astrophysics of Minimal Dark Matter," Nucl. Phys. B787 (2007) 152-175, arXiv:0706.4071 [hep-ph].

[58] M. Cirelli and A. Strumia, "Minimal Dark Matter: Model and results," New J. Phys. 11 (2009) 105005, arXiv:0903.3381 [hep-ph]. 
[59] A. Birkedal, A. Noble, M. Perelstein, and A. Spray, "Little Higgs dark matter," Phys. Rev. D74 (2006) 035002, arXiv:hep-ph/0603077 [hep-ph].

[60] A. Martin, "Dark matter in the simplest little Higgs model," arXiv:hep-ph/0602206 [hep-ph].

[61] M. Perelstein and A. Spray, "Indirect Detection of Little Higgs Dark Matter," Phys. Rev. D75 (2007) 083519, arXiv:hep-ph/0610357 [hep-ph].

[62] P. Cushman et al., "Working Group Report: WIMP Dark Matter Direct Detection," in Community Summer Study 2013: Snowmass on the Mississippi (CSS2013) Minneapolis, MN, USA, July 29-August 6, 2013. 2013. arXiv:1310.8327 [hep-ex]

[63] L. Baudis, "Dark matter detection," J. Phys. G43 (2016) no. 4, 044001.

[64] SuperCDMS Collaboration, R. Agnese et al., "Improved WIMP-search reach of the CDMS II germanium data," Phys. Rev. D92 (2015) no. 7, 072003, arXiv:1504.05871 [hep-ex].

[65] EDELWEISS Collaboration, E. Armengaud et al., "A search for low-mass WIMPs with EDELWEISS-II heat-and-ionization detectors," Phys. Rev. D86 (2012) 051701, arXiv:1207.1815 [astro-ph.CO].

[66] CoGeNT Collaboration, C. E. Aalseth et al., "CoGeNT: A Search for Low-Mass Dark Matter using p-type Point Contact Germanium Detectors," Phys. Rev. D88 (2013) 012002 arXiv:1208.5737 [astro-ph.CO].

[67] LUX Collaboration, D. S. Akerib et al., "The Large Underground Xenon (LUX)

Experiment," Nucl. Instrum. Meth. A704 (2013) 111-126, arXiv:1211.3788 [physics.ins-det].

[68] XENON1T Collaboration, E. Aprile, "The XENON1T Dark Matter Search Experiment," Springer Proc. Phys. 148 (2013) 93-96, arXiv:1206.6288 [astro-ph.IM]

[69] DEAP Collaboration, M. G. Boulay, "DEAP-3600 Dark Matter Search at SNOLAB," J. Phys. Conf. Ser. 375 (2012) 012027, arXiv:1203.0604 [astro-ph.IM],

[70] S. Arrenberg et al., "Working Group Report: Dark Matter Complementarity," in Community Summer Study 2013: Snowmass on the Mississippi (CSS2013) Minneapolis, MN, USA, July 29-August 6, 2013. 2013. arXiv:1310.8621 [hep-ph]. 
[71] PAMELA Collaboration, P. Picozza, "Five Years of PAMELA in Orbit," in Proceedings, 32nd International Cosmic Ray Conference (ICRC 2011), vol. c, p. 167. 2011.

[72] AMS-02 Collaboration, V. Vagelli and Z. L. Weng, "The $\left(e^{+}+e\right)$ flux measurement up to 1 TeV with the AMS-02 experiment," Nucl. Part. Phys. Proc. 273-275 (2016) 2369-2371.

[73] IceCube Collaboration, M. G. Aartsen et al., "All-flavour Search for Neutrinos from Dark Matter Annihilations in the Milky Way with IceCube/DeepCore," arXiv:1606.00209 [astro-ph.HE].

[74] Fermi-LAT Collaboration, M. Ajello et al., "Fermi-LAT Observations of High-Energy $\gamma$-Ray Emission Toward the Galactic Center," Astrophys. J. 819 (2016) no. 1, 44, arXiv:1511.02938 [astro-ph.HE].

[75] L. Goodenough and D. Hooper, "Possible Evidence For Dark Matter Annihilation In The Inner Milky Way From The Fermi Gamma Ray Space Telescope," arXiv:0910.2998 [hep-ph],

[76] Fermi-LAT Collaboration, V. Vitale and A. Morselli, "Indirect Search for Dark Matter from the center of the Milky Way with the Fermi-Large Area Telescope," in Fermi gamma-ray space telescope. Proceedings, 2nd Fermi Symposium, Washington, USA, November 2-5, 2009. 2009. arXiv:0912.3828 [astro-ph.HE].

[77] C. Gordon and O. Macias, "Dark Matter and Pulsar Model Constraints from Galactic Center Fermi-LAT Gamma Ray Observations," Phys. Rev. D88 (2013) no. 8, 083521 , arXiv:1306.5725 [astro-ph.HE]. [Erratum: Phys. Rev.D89,no.4,049901(2014)].

[78] P. J. Fox, R. Harnik, J. Kopp, and Y. Tsai, "LEP Shines Light on Dark Matter," Phys. Rev. D84 (2011) 014028, arXiv:1103.0240 [hep-ph].

[79] ATLAS, CMS Collaboration, A. de Cosa, "LHC results for dark matter from ATLAS and CMS," in 12th Conference on the Intersections of Particle and Nuclear Physics (CIPANP 2015) Vail, Colorado, USA, May 19-24, 2015. 2015. arXiv:1510.01516 [hep-ex].

[80] ATLAS, CMS Collaboration, D. Zerwas, "Searches for Dark Matter in ATLAS and CMS," PoS EPS-HEP2015 (2015) 150.

[81] J. Frost, "Overview of Dark Matter Searches with Mono-X signatures at ATLAS,". https://cds.cern.ch/record/1980088. 
[82] ATLAS, CMS Collaboration, A. Cortes Gonzalez, "Searches for mono-X at the LHC," PoS DIS2014 (2014) 116.

[83] M. Cirelli, T. Hambye, P. Panci, F. Sala, and M. Taoso, "Gamma ray tests of Minimal Dark Matter," JCAP 1510 (2015) no. 10, 026, arXiv:1507.05519 [hep-ph].

[84] T. Hambye, F. S. Ling, L. Lopez Honorez, and J. Rocher, "Scalar Multiplet Dark Matter," JHEP 07 (2009) 090, arXiv:0903.4010 [hep-ph]. [Erratum: JHEP05,066(2010)].

[85] C. Garcia-Cely, A. Ibarra, A. S. Lamperstorfer, and M. H. G. Tytgat, "Gamma-rays from Heavy Minimal Dark Matter," JCAP 1510 (2015) no. 10, 058, arXiv: 1507.05536 [hep-ph],

[86] H. Georgi, "Lie algebras in particle physics," Front. Phys. 54 (1999) 1-320.

[87] J. G. Belinfante, B. Kolman, and H. A. Smith, "An introduction to Lie groups and Lie algebras, with applications," SIAM Rev. 8 (1965) 11-46.

[88] A. P. Balachandran, G. Marmo, B. S. Skagerstam, and A. Stern, "Gauge Symmetries and Fiber Bundles: Applications to Particle Dynamics," Lect. Notes Phys. 188 (1983) 1-140.

[89] E. Noether, "Invariant Variation Problems," Gott. Nachr. 1918 (1918) 235-257, arXiv:physics/0503066 [physics]. [Transp. Theory Statist. Phys.1,186(1971)].

[90] M. Bañados and I. A. Reyes, “A short review on Noether's theorems, gauge symmetries and boundary terms, for students," arXiv:1601.03616 [hep-th].

[91] D. B. Lichtenberg, Unitary symmetry and elementary particles. Academic Press, New York, USA, 1970.

[92] P. Ramond, Group theory: A physicist's survey. Cambridge Univeristy Press, Cambridge, UK, 2010.

[93] S. P. Martin, "A Supersymmetry primer," arXiv:hep-ph/9709356 [hep-ph]. [Adv. Ser. Direct. High Energy Phys.18,1(1998)].

[94] E. Ma, "Pathways to naturally small neutrino masses," Phys. Rev. Lett. 81 (1998) 1171-1174, arXiv:hep-ph/9805219 [hep-ph].

[95] V. Silveira and A. Zee, "SCALAR PHANTOMS," Phys. Lett. B161 (1985) 136-140.

[96] M. J. G. Veltman and F. J. Yndurain, "RADIATIVE CORRECTIONS TO W W SCATTERING," Nucl. Phys. B325 (1989) 1-17. 
[97] J. McDonald, "Gauge singlet scalars as cold dark matter," Phys. Rev. D50 (1994) 3637-3649, arXiv:hep-ph/0702143 [HEP-PH].

[98] C. P. Burgess, M. Pospelov, and T. ter Veldhuis, "The Minimal model of nonbaryonic dark matter: A Singlet scalar," Nucl. Phys. B619 (2001) 709-728, arXiv:hep-ph/0011335 [hep-ph].

[99] N. G. Deshpande and E. Ma, "Pattern of Symmetry Breaking with Two Higgs Doublets," Phys. Rev. D18 (1978) 2574.

[100] T. Araki, C. Q. Geng, and K. I. Nagao, "Dark Matter in Inert Triplet Models," Phys. Rev. D83 (2011) 075014, arXiv:1102.4906 [hep-ph].

[101] J. M. Cline, K. Kainulainen, P. Scott, and C. Weniger, "Update on scalar singlet dark matter," Phys. Rev. D88 (2013) 055025, arXiv:1306.4710 [hep-ph]. [Erratum: Phys. Rev.D92,no.3,039906(2015)].

[102] S. Y. Ayazi and S. M. Firouzabadi, "Constraining Inert Triplet Dark Matter by the LHC and FermiLAT," JCAP 1411 (2014) no. 11, 005, arXiv: 1408.0654 [hep-ph].

[103] G. Belanger, B. Dumont, A. Goudelis, B. Herrmann, S. Kraml, and D. Sengupta, "Dilepton constraints in the Inert Doublet Model from Run 1 of the LHC," Phys. Rev. D91 (2015) no. 11, 115011, arXiv:1503.07367 [hep-ph].

[104] W. J. Marciano, G. Valencia, and S. Willenbrock, "Renormalization Group Improved Unitarity Bounds on the Higgs Boson and Top Quark Masses," Phys. Rev. D40 (1989) 1725.

[105] S. Burdin, M. Fairbairn, P. Mermod, D. Milstead, J. Pinfold, T. Sloan, and W. Taylor, "Non-collider searches for stable massive particles," Phys. Rept. 582 (2015) 1-52, arXiv:1410.1374 [hep-ph].

[106] A. Alloul, N. D. Christensen, C. Degrande, C. Duhr, and B. Fuks, "FeynRules 2.0 - A complete toolbox for tree-level phenomenology," Comput. Phys. Commun. 185 (2014) 2250-2300, arXiv:1310.1921 [hep-ph].

[107] C. Degrande, C. Duhr, B. Fuks, D. Grellscheid, O. Mattelaer, and T. Reiter, "UFO - The Universal FeynRules Output," Comput. Phys. Commun. 183 (2012) 1201-1214. arXiv:1108.2040 [hep-ph]. 
[108] J. Alwall, C. Duhr, B. Fuks, O. Mattelaer, D. G. Özturk, and C.-H. Shen, "Computing decay rates for new physics theories with FeynRules and MadGraph 5 aMC@NLO," Comput. Phys. Commun. 197 (2015) 312-323, arXiv:1402.1178 [hep-ph].

[109] J. Alwall, R. Frederix, S. Frixione, V. Hirschi, F. Maltoni, O. Mattelaer, H. S. Shao, T. Stelzer, P. Torrielli, and M. Zaro, "The automated computation of tree-level and next-to-leading order differential cross sections, and their matching to parton shower simulations," JHEP 07 (2014) 079, arXiv:1405.0301 [hep-ph].

[110] E. Wigner, "Unreasonable effectiveness of mathematics in the natural sciences," Communications in Pure and Applied Mathematics 13 (1960) 1-14.

[111] D. J. Gross and F. Wilczek, "Asymptotically Free Gauge Theories. 1," Phys. Rev. D8 (1973) $3633-3652$.

[112] M. E. Peskin and D. V. Schroeder, An Introduction to Quantum Field Theory. Addison-Wesley Publishing Company, Reading, USA, 1995.

[113] T. D. Lee, "A Theory of Spontaneous T Violation," Phys. Rev. D8 (1973) 1226-1239. $[, 516(1973)]$.

[114] G. C. Branco, P. M. Ferreira, L. Lavoura, M. N. Rebelo, M. Sher, and J. P. Silva, "Theory and phenomenology of two-Higgs-doublet models," Phys. Rept. 516 (2012) 1-102, arXiv:1106.0034 [hep-ph].

[115] A. Arhrib, R. Benbrik, M. Chabab, G. Moultaka, M. C. Peyranere, L. Rahili, and J. Ramadan, "The Higgs Potential in the Type II Seesaw Model," Phys. Rev. D84 (2011) 095005, arXiv:1105.1925 [hep-ph].

[116] K. Hartling, K. Kumar, and H. E. Logan, "The decoupling limit in the Georgi-Machacek model," Phys. Rev. D90 (2014) no. 1, 015007, arXiv:1404.2640 [hep-ph].

[117] T. P. Cheng, E. Eichten, and L.-F. Li, "Higgs Phenomena in Asymptotically Free Gauge Theories," Phys. Rev. D9 (1974) 2259.

[118] A. Goudelis, B. Herrmann, and O. Stål, "Dark matter in the Inert Doublet Model after the discovery of a Higgs-like boson at the LHC," JHEP 09 (2013) 106, arXiv: 1303.3010 [hep-ph]. 
[119] H. Georgi and M. Machacek, "DOUBLY CHARGED HIGGS BOSONS," Nucl. Phys. B262 (1985) 463-477.

[120] M. S. Chanowitz and M. Golden, "Higgs Boson Triplets With $M_{W}=M_{Z} \cos \theta_{W}$," Phys. Lett. B165 (1985) 105-108

[121] M.-x. Luo, H.-w. Wang, and Y. Xiao, "Two loop renormalization group equations in general gauge field theories," Phys. Rev. D67 (2003) 065019, arXiv:hep-ph/0211440 [hep-ph].

[122] Y. Hamada, K. Kawana, and K. Tsumura, "Landau pole in the Standard Model with weakly interacting scalar fields," Phys. Lett. B747 (2015) 238-244, arXiv:1505.01721 [hep-ph].

[123] G. Giacomelli and R. Giacomelli, "The LEP legacy," in Non-accelerator astroparticle physics. Proceedings, 7th School, Trieste, Italy, July 26-August 6, 2004, pp. 218-232. 2005. arXiv:hep-ex/0503050 [hep-ex].

[124] C. O'Luanaigh, The Large Electron-Positron Collider, July, 2012. http://cds.cern.ch/record/1997351.

[125] A. Levy, "The Legacy of HERA: The First decade," in Deep inelastic scattering. Proceedings, 8th International Workshop, DIS 2000, Liverpool, UK, April 25-30, 2000, pp. 645-654. 2000. arXiv:hep-ph/0008130 [hep-ph]. http://alice.cern.ch/format/showfull?sysnb=2203292.

[126] D. Froidevaux and P. Jenni, "PHYSICS AT THE IMPROVED CERN anti-p p COLLIDER," Adv. Ser. Direct. High Energy Phys. 4 (1989) 323-368.

[127] CDF, Do Collaboration, Y. Peters, "Tevatron Legacy," Nuovo Cim. C035N3 (2012) 181-186, arXiv:1202.6196 [hep-ex].

[128] D. d'Enterria, "Physics at the LHC: A Short overview," J. Phys. Conf. Ser. 270 (2011) 012001, arXiv: 1010.1491 [hep-ex].

[129] C. O'Luanaigh, The Large Hadron Collider, January, 2014. http://cds.cern.ch/record/1998498.

[130] D. G. Charlton, "LEP, SLC and the standard model," eConf C020805 (2002) TW05, arXiv:hep-ex/0211003 [hep-ex].

[131] G. Okham, "Particle interactions with parts of the detector." Lecture notes (Physics 5601, Carleton University), November 16, 2011. 
[132] ATLAS Collaboration, D. Ferrère, "The upgrade of the ATLAS Inner Detector," Nucl. Instrum. Meth. A718 (2013) 30-38

[133] CMS Collaboration, V. Veszpremi, "Operation and performance of the CMS tracker," JINST 9 (2014) C03005, arXiv:1402.0675 [physics.ins-det].

[134] Y. Nagashima, Elementary particle physics, vol. 1. Wiley-VCH, Weinheim, Germany, 2010.

[135] V. D. Barger and R. J. N. Phillips, Collider Physics. No. 71 in Frontiers in Physics. Addison-Wesley Publishing Company, Redwood City, USA, 1987.

[136] A. Papaefstathiou, "How-to: Write a parton-level Monte Carlo event generator," arXiv:1412.4677 [hep-ph].

[137] J. Alwall, M. Herquet, F. Maltoni, O. Mattelaer, and T. Stelzer, "MadGraph 5 : Going Beyond," JHEP 06 (2011) 128, arXiv:1106.0522 [hep-ph]

[138] T. Sjostrand, S. Mrenna, and P. Z. Skands, "PYTHIA 6.4 Physics and Manual," JHEP 05 (2006) 026, arXiv:hep-ph/0603175 [hep-ph].

[139] DELPHES 3 Collaboration, J. de Favereau, C. Delaere, P. Demin, A. Giammanco, V. Lemaître, A. Mertens, and M. Selvaggi, "DELPHES 3, A modular framework for fast simulation of a generic collider experiment," JHEP 02 (2014) 057, arXiv:1307.6346 [hep-ex]

[140] M. Beltran, D. Hooper, E. W. Kolb, Z. A. C. Krusberg, and T. M. P. Tait, "Maverick dark matter at colliders," JHEP 09 (2010) 037, arXiv: 1002.4137 [hep-ph].

[141] J. Goodman, M. Ibe, A. Rajaraman, W. Shepherd, T. M. P. Tait, and H.-B. Yu, "Constraints on Dark Matter from Colliders," Phys. Rev. D82 (2010) 116010, arXiv: 1008.1783 [hep-ph].

[142] ATLAS Collaboration, "Search for direct-slepton and direct-chargino production in final states with two opposite-sign leptons, missing transverse momentum and no jets in $20 / \mathrm{fb}$ of pp collisions at sqrt(s) $=8 \mathrm{TeV}$ with the ATLAS detector," ATLAS-CONF-2013-049.

[143] ATLAS Collaboration, "Search for direct production of charginos and neutralinos in events with three leptons and missing transverse momentum in $21 \mathrm{fb}^{-1}$ of pp collisions at $\sqrt{s}=8 \mathrm{TeV}$ with the ATLAS detector," ATLAS-CONF-2013-035. 
[144] ATLAS Collaboration, G. Aad et al., "Search for direct production of charginos and neutralinos in events with three leptons and missing transverse momentum in $\sqrt{s}=8 \mathrm{TeV} p p$ collisions with the ATLAS detector," JHEP 04 (2014) 169, arXiv:1402.7029 [hep-ex].

[145] ATLAS Collaboration, "Search for supersymmetry in events with four or more leptons in $21 \mathrm{fb}^{-1}$ of pp collisions at $\sqrt{s}=8 \mathrm{TeV}$ with the ATLAS detector," ATLAS-CONF-2013-036.

[146] ATLAS Collaboration, "Search for strongly produced supersymmetric particles in decays with two leptons at $\sqrt{s}=8 \mathrm{TeV}, "$ ATLAS-CONF-2013-089.

[147] ATLAS Collaboration, "Search for electroweak production of supersymmetric particles in final states with at least two hadronically decaying taus and missing transverse momentum with the ATLAS detector in proton-proton collisions at $\sqrt{s}=8 \mathrm{TeV}, "$ ATLAS-CONF-2013-028.

[148] ATLAS Collaboration, "Search for strongly produced superpartners in final states with two same sign leptons with the ATLAS detector using $21 \mathrm{fb}^{-1}$ of proton-proton collisions at $\sqrt{s}=8 \mathrm{TeV} ., "$ ATLAS-CONF-2013-007.

[149] ATLAS Collaboration, "Search for New Phenomena in Monojet plus Missing Transverse Momentum Final States using $10 \mathrm{fb}^{-1}$ of pp Collisions at $\sqrt{s}=8 \mathrm{TeV}$ with the ATLAS detector at the LHC," ATLAS-CONF-2012-147.

[150] ATLAS Collaboration, "Search for pair-produced top squarks decaying into a charm quark and the lightest neutralinos with $20.3 \mathrm{fb}^{-1}$ of $p p$ collisions at $\sqrt{s}=8 \mathrm{TeV}$ with the ATLAS detector at the LHC," ATLAS-CONF-2013-068.

[151] ATLAS Collaboration, "Search for squarks and gluinos with the ATLAS detector in final states with jets and missing transverse momentum and $20.3 \mathrm{fb}^{-1}$ of $\sqrt{s}=8 \mathrm{TeV}$ proton-proton collision data," ATLAS-CONF-2013-047.

[152] CMS Collaboration, "Search for electroweak production of charginos, neutralinos, and sleptons using leptonic final states in pp collisions at $8 \mathrm{TeV}$," CMS-PAS-SUS-13-006.

[153] CMS Collaboration, "Search for new physics in monojet events in pp collisions at sqrt(s)=8 TeV," CMS-PAS-EXO-12-048.

[154] T. A. W. Martin and D. Morrissey, "Electroweakino constraints from LHC data," JHEP 12 (2014) 168, arXiv:1409.6322 [hep-ph]. 
[155] J. Alwall, S. Höche, F. Krauss, N. Lavesson, L. Lönnbald, F. Maltoni, M. Mangano, M. Moretti, C. Papadopoulos, Piccinini, S. Schumann, M. Trecani, J. Winter, and M. Worek, "Comparative study of various algorithms for the merging of parton showers and matrix elements in hadronic collisions," Eur. Phys. J. C53 (2008) 473-500, arXiv:0706.2569 [hep-ph]

[156] S. Catani, F. Krauss, R. Kuhn, and B. R. Webber, "QCD matrix elements + parton showers," JHEP 11 (2001) 063, arXiv:hep-ph/0109231 [hep-ph].

[157] P. Lenzi and J. M. Butterworth, "A Study on Matrix Element corrections in inclusive Z/ gamma* production at LHC as implemented in PYTHIA, HERWIG, ALPGEN and SHERPA," arXiv:0903.3918 [hep-ph]

[158] T. A. W. Martin, "Seer: An analysis package for LHCO files," arXiv:1503.03073 [hep-ph]

[159] ATLAS Collaboration, "Performance assumptions for an upgraded ATLAS detector at a High-Luminosity LHC," ATL-PHYS-PUB-2013-004. https://cds.cern.ch/record/1527529.

[160] J. Hisano and K. Tsumura, "Higgs boson mixes with an SU(2) septet representation," Phys. Rev. D87 (2013) 053004, arXiv:1301.6455 [hep-ph].

[161] C. Alvarado, L. Lehman, and B. Ostdiek, "Surveying the Scope of the $S U(2)_{L}$ Scalar Septet Sector," JHEP 05 (2014) 150, arXiv: 1404.3208 [hep-ph].

[162] M. Bonvini, S. Marzani, C. Muselli, and L. Rottoli, "On the Higgs cross section at $\mathrm{N}^{3} \mathrm{LO}+\mathrm{N}^{3} \mathrm{LL}$ and its uncertainty," arXiv:1603.08000 [hep-ph].

[163] I. Maksymyk, C. P. Burgess, and D. London, "Beyond S, T and U," Phys. Rev. D50 (1994) 529-535, arXiv:hep-ph/9306267 [hep-ph].

[164] M. E. Peskin and T. Takeuchi, "A New constraint on a strongly interacting Higgs sector," Phys. Rev. Lett. 65 (1990) 964-967.

[165] M. Baak, M. Goebel, J. Haller, A. Hoecker, D. Kennedy, R. Kogler, K. Moenig, M. Schott, and J. Stelzer, "The Electroweak Fit of the Standard Model after the Discovery of a New Boson at the LHC," Eur. Phys. J. C72 (2012) 2205, arXiv:1209.2716 [hep-ph].

[166] L. Lavoura and L.-F. Li, "Making the small oblique parameters large," Phys. Rev. D49 (1994) 1409-1416, arXiv:hep-ph/9309262 [hep-ph] 
[167] Y. Cai, W. Chao, and S. Yang, "Scalar Septuplet Dark Matter and Enhanced $h \rightarrow \gamma \gamma$ Decay Rate,"JHEP 12 (2012) 043 arXiv:1208.3949 [hep-ph].

[168] C.-S. Chen, C.-Q. Geng, D. Huang, and L.-H. Tsai, "Many high-charged scalars in LHC searches and Majorana neutrino mass generations," Phys. Rev. D87 (2013) no. 7, 077702, arXiv:1212.6208 [hep-ph].

[169] J. F. Gunion, H. E. Haber, G. L. Kane, and S. Dawson, "The Higgs Hunter's Guide," Front. Phys. 80 (2000) 1-448.

[170] ATLAS Collaboration, "Measurements of the properties of the Higgs-like boson in the two photon decay channel with the ATLAS detector using $25 \mathrm{fb}^{-1}$ of proton-proton collision data," ATLAS-CONF-2013-012.

[171] CMS Collaboration, "Updated measurements of the Higgs boson at $125 \mathrm{GeV}$ in the two photon decay channel," CMS-PAS-HIG-13-001.

[172] ATLAS Collaboration, "Search for the Standard Model Higgs boson in the $H \rightarrow Z \gamma$ decay mode with pp collisions at $\sqrt{s}=7$ and $8 \mathrm{TeV}, "$ ATLAS-CONF-2013-009.

[173] CMS Collaboration, S. Chatrchyan et al., "Search for a Higgs boson decaying into a Z and a photon in pp collisions at $\sqrt{s}=7$ and $8 \mathrm{TeV}$," Phys. Lett. B726 (2013) 587-609 arXiv:1307.5515 [hep-ex].

[174] C.-S. Chen, C.-Q. Geng, D. Huang, and L.-H. Tsai, "New Scalar Contributions to $h \rightarrow Z \gamma$," Phys. Rev. D87 (2013) 075019, arXiv:1301.4694 [hep-ph].

[175] M. Carena, I. Low, and C. E. M. Wagner, "Implications of a Modified Higgs to Diphoton Decay Width," JHEP 08 (2012) 060, arXiv:1206.1082 [hep-ph].

[176] E. W. Kolb and M. S. Turner, "The Early Universe," Front. Phys. 69 (1990) 1-547.

[177] A. Liddle, An introduction to modern cosmology. Wiley-VCH, Weinheim, Germany, 2009.

[178] W. Hu, Intermediate Guide to the Acoustic Peaks and Polarization, 2011. http://background.uchicago.edu/ whu/intermediate/intermediate.html.

[179] W. Hu and S. Dodelson, "Cosmic microwave background anisotropies," Ann. Rev. Astron. Astrophys. 40 (2002) 171-216, arXiv:astro-ph/0110414 [astro-ph].

[180] C. Pitrou, CMBquick, 2009. http://www2.iap.fr/users/pitrou/cmbquick.htm. 
[181] WMAP Collaboration, E. Komatsu et al., "Five-Year Wilkinson Microwave Anisotropy Probe (WMAP) Observations: Cosmological Interpretation," Astrophys. J. Suppl. 180 (2009) 330-376, arXiv:0803.0547 [astro-ph]

[182] P. Gondolo and G. Gelmini, "Cosmic abundances of stable particles: Improved analysis," Nucl. Phys. B360 (1991) 145-179.

[183] G. Steigman, B. Dasgupta, and J. F. Beacom, "Precise Relic WIMP Abundance and its Impact on Searches for Dark Matter Annihilation," Phys. Rev. D86 (2012) 023506 , arXiv: 1204.3622 [hep-ph].

[184] A. Belyaev, N. D. Christensen, and A. Pukhov, "CalcHEP 3.4 for collider physics within and beyond the Standard Model," Comput. Phys. Commun. 184 (2013) 1729-1769, arXiv:1207.6082 [hep-ph].

[185] D. J. Griffiths, Introduction to Quantum Mechanics. Pearson Education, Inc., Upper Saddle River, USA, 2nd ed., 2005.

[186] A. Sommerfeld, "Über die beugung und bremsung der elektronen," Annalen der Physik 403 (1931) no. 3, 257-330.

[187] I. Harris and L. M. Brown, "Radiative Corrections to Pair Annihilation," Phys. Rev. 105 (1957) 1656-1661.

[188] R. R. Lewis and G. W. Ford, "Coulomb Effects in Inner Bremsstrahlen," Phys. Rev. 107 (1957) 756-765.

[189] J. Hisano, S. Matsumoto, and M. M. Nojiri, "Explosive dark matter annihilation," Phys. Rev. Lett. 92 (2004) 031303, arXiv:hep-ph/0307216 [hep-ph].

[190] N. Arkani-Hamed, D. P. Finkbeiner, T. R. Slatyer, and N. Weiner, "A Theory of Dark Matter," Phys. Rev. D79 (2009) 015014, arXiv:0810.0713 [hep-ph].

[191] L. Hulthén Ark. Mat. Astron. Fys. 28A (1942) 5.

[192] L. Hulthén, "Über die Eigenlösungen der Schrödinger-Gleichung der Deuterons," Ark. Mat. Astron. Fys. 29B (1942) 1-12.

[193] T. R. Slatyer, "The Sommerfeld enhancement for dark matter with an excited state," JCAP 1002 (2010) 028, arXiv:0910.5713 [hep-ph]. 
[194] S. Cassel, "Sommerfeld factor for arbitrary partial wave processes," J. Phys. G37 (2010) 105009, arXiv:0903.5307 [hep-ph].

[195] J. Hisano, S. Matsumoto, M. M. Nojiri, and O. Saito, "Non-perturbative effect on dark matter annihilation and gamma ray signature from galactic center," Phys. Rev. D71 (2005) 063528, arXiv:hep-ph/0412403 [hep-ph].

[196] M. Laine and K. Rummukainen, "What's new with the electroweak phase transition?," Nucl. Phys. Proc. Suppl. 73 (1999) 180-185, arXiv:hep-lat/9809045 [hep-lat].

[197] M. Laine and M. Meyer, "Standard Model thermodynamics across the electroweak crossover," JCAP 1507 (2015) no. 07, 035, arXiv: 1503.04935 [hep-ph].

[198] A. Strumia, "Sommerfeld corrections to type-II and III leptogenesis," Nucl. Phys. B809 (2009) 308-317, arXiv:0806.1630 [hep-ph].

[199] C. Garcia-Cely, M. Gustafsson, and A. Ibarra, "Probing the Inert Doublet Dark Matter Model with Cherenkov Telescopes," JCAP 1602 (2016) no. 02, 043, arXiv:1512.02801 [hep-ph].

[200] SuperCDMS Collaboration, R. Agnese et al., "New Results from the Search for Low-Mass Weakly Interacting Massive Particles with the CDMS Low Ionization Threshold Experiment," Phys. Rev. Lett. 116 (2016) no. 7, 071301, arXiv:1509.02448 [astro-ph.CO].

[201] LUX Collaboration, D. S. Akerib et al., "Improved Limits on Scattering of Weakly Interacting Massive Particles from Reanalysis of 2013 LUX Data," Phys. Rev. Lett. 116 (2016) no. 16, 161301, arXiv:1512.03506 [astro-ph.C0].

[202] DEAP Collaboration, P. A. Amaudruz et al., "DEAP-3600 Dark Matter Search,"] in Proceedings, 37th International Conference on High Energy Physics (ICHEP 2014), vol. 273-275, pp. 340-346. 2016. arXiv:1410.7673 [physics.ins-det].

[203] XENON Collaboration, E. Aprile et al., "Physics reach of the XENON1T dark matter experiment," JCAP 1604 (2016) no. 04, 027, arXiv:1512.07501 [physics.ins-det].

[204] L. Baudis, "WIMP Dark Matter Direct-Detection Searches in Noble Gases," Phys. Dark Univ. 4 (2014) 50-59, arXiv: 1408.4371 [astro-ph.IM]. 
[205] T. Marrodán Undagoitia and L. Rauch, "Dark matter direct-detection experiments," J. Phys. G43 (2016) no. 1, 013001, arXiv:1509.08767 [physics.ins-det].

[206] XMASS Collaboration, M. Yamashita, "Direct Dark Matter Search with XMASS-I," in 12th Conference on the Intersections of Particle and Nuclear Physics (CIPANP 2015) Vail, Colorado, USA, May 19-24, 2015. 2015. arXiv:1511.07597 [astro-ph.C0].

[207] ArDM Collaboration, A. Marchionni et al., "ArDM: a ton-scale LAr detector for direct Dark Matter searches," J. Phys. Conf. Ser. 308 (2011) 012006, arXiv:1012.5967 [physics.ins-det].

[208] A. Desai and A. Moskowitz, DMTools: Dark matter limit plot generator, 2016. http://dmtools.brown.edu.

[209] J. R. Ellis, A. Ferstl, and K. A. Olive, "Reevaluation of the elastic scattering of supersymmetric dark matter," Phys. Lett. B481 (2000) 304-314, arXiv:hep-ph/0001005 [hep-ph]

[210] H.-Y. Cheng, "Scalar and Pseudoscalar Higgs Couplings with Nucleons," Nucl. Phys. Proc. Suppl. 246-247 (2014) 109-115

[211] LZ Collaboration, D. N. McKinsey, "The LZ dark matter experiment," J. Phys. Conf. Ser. 718 (2016) no. 4, 042039

[212] J. Aalbers et al., "DARWIN: towards the ultimate dark matter detector," arXiv:1606.07001 [astro-ph.IM].

[213] J. Billard, L. Strigari, and E. Figueroa-Feliciano, "Implication of neutrino backgrounds on the reach of next generation dark matter direct detection experiments," Phys. Rev. D89 (2014) no. 2, 023524, arXiv:1307.5458 [hep-ph].

[214] LUX Collaboration, D. S. Akerib et al., "Results from a search for dark matter in LUX with 332 live days of exposure," arXiv:1608.07648 [astro-ph.C0].

[215] PandaX-II Collaboration, A. Tan et al., "Dark Matter Results from First 98.7-day Data of PandaX-II Experiment," arXiv:1607.07400 [hep-ex].

[216] H. E. Logan and V. Rentala, "All the generalized Georgi-Machacek models," Phys. Rev. D92 (2015) no. 7, 075011, arXiv:1502.01275 [hep-ph]. 
[217] C. S. Wu, "The Universal Fermi Interaction and the Conserved Vector Current in Beta Decay," Rev. Mod. Phys. 36 (1964) 618.

[218] K. S. Krane, INTRODUCTORY NUCLEAR PHYSICS. Wiley, New York, USA, 1987.

[219] XENON100 Collaboration, E. Aprile et al., "Dark Matter Results from 225 Live Days of XENON100 Data," Phys. Rev. Lett. 109 (2012) 181301, arXiv:1207.5988 [astro-ph.C0]

[220] ATLAS Collaboration, "Observation of an excess of events in the search for the Standard Model Higgs boson in the gamma-gamma channel with the ATLAS detector," ATLAS-CONF-2012-091.

[221] CMS Collaboration, "Evidence for a new state decaying into two photons in the search for the standard model Higgs boson in pp collisions," CMS-PAS-HIG-12-015.

[222] S. Dittmaier et al., "Handbook of LHC Higgs Cross Sections: 2. Differential Distributions," arXiv:1201.3084 [hep-ph]. 